\section{Acute Pancreatitis - Basic}

\section{AP-B1 \\ Inhibition of Cyclophilin-D Prevents Necrosis in Acute Pancreatitis Through the Reduction of Mitochondrial Injury}

\section{R. Mukherjee, W. Huang, A. Gukovskya, D.N. Criddle, R. Sutton \\ NIHR Pancreas Biomedical Research Unit, Royal Liverpool University Hospital, UK; Physiological Laboratory, University of Liverpool, UK; VAGLA and University of California, Los Angeles, USA}

Introduction: Calcium overload causing mitochondrial damage determines acinar cell fate in acute pancreatitis. Cyclophilin-D (CypD) modulates the mitochondrial permeability transition pore (mPTP), determining cell fate in response to injury. We have investigated the role of the MPTP in pancreatitis using genetic (Cyp-D KO) and pharmacological (Alisporivir, DEB025) inhibition of Cyp-D. Methods: Confocal fluorescence microscopy of isolated pancreatic acinar cells from Ppif-/- and wild type mice was used to measure cytosolic calcium $([\mathrm{Ca} 2+] \mathrm{c}), \mathrm{NAD}(\mathrm{P}) \mathrm{H}$ autofluorescence, mitochondrial membrane potential $(\Delta \psi \mathrm{m})$ and cell death in response to taurolithocholic acid 3-sulphate (TLC-S) or CCK-8 hyperstimulation. Murine pancreatitis was induced by ductal bile acid infusion or cerulein injections $(7 \times 50 \mu \mathrm{g} / \mathrm{kg}$ ip) and DEB025 $(10 \mathrm{mg} / \mathrm{kg}$ or $100 \mathrm{mg} / \mathrm{kg}$ after disease induction) tested as treatment, using standardised assessment of plasma amylase, IL6 and pancreatic histopathology ( $\geq 6$ all groups). Results: TLC-S or CCK- 8 induced marked elevation in $[\mathrm{Ca} 2+] \mathrm{c}$, falls in $\Delta \psi \mathrm{m}$ and NADH levels, and acinar cell necrosis pathway activation. Cyp-D KO preserved cell function and reduced necrosis $(p<0.05)$ but not apoptosis pathway activation. The severity of pancreatitis in both models was greatly reduced (all parameters $\mathrm{p}<0.05$ ) in Cyp-D KO mice and DEBO25-treated wild type mice. Conclusion: Inhibition of Cyp-D preserves mitochondrial function and protects pancreatic acinar cells from pancreatitis toxins, an approach that has potential as a treatment for acute pancreatitis.

\section{AP-B2 \\ A Novel Model of Acute Alcoholic Pancreatitis by Concomitant Administration of Ethanol and Fatty Acid}

\author{
W. Huang, R. Mukherjee, V. Elliott, D. M. Booth, A. Tepikin, \\ D. N. Criddle, R. Sutton \\ Division of Surgery and Oncology and NIHR Pancreas \\ Biomedical Research Unit, Royal Liverpool University \\ Hospital, Liverpool, UK; Physiological Laboratory, \\ University of Liverpool, Liverpool, UK
}

Introduction: At present no simple, reliable model of murine alcoholic pancreatitis exists. Non-oxidative metabolites of alcohol, fatty acid ethyl esters, are synthesized in the pancreas by enzymes which conjugate ethanol (EtOH) to fatty acids and have been implicated as mediators of acute pancreatitis. We have characterised a novel acute pancreatitis model induced by concomitant administration of EtOH and fatty acid (palmitoleic acid: POA) in mice. Methods: CD1 mice (30-35 g) were injected intraperitoneally twice one $\mathrm{h}$ apart, either with normal saline $(50 \mu \mathrm{l})$, or $1.32 \mathrm{~g} / \mathrm{kg}$ pure $\mathrm{EtOH}$, or $1.32 \mathrm{~g} / \mathrm{kg} \mathrm{EtOH}+0.25 \mathrm{mg} / \mathrm{kg}$ POA, or $1.32 \mathrm{~g} / \mathrm{kg} \mathrm{EtOH}+2 \mathrm{mg} / \mathrm{kg}$ POA. The severity of acute pancreatitis was assessed at 2, 6, 12, 24 and $48 \mathrm{~h}$ after the first injection. The peak serum EtOH concentration was quantified by enzymatic assay. Results: The peak serum EtOH concentration achieved was $35.6 \pm 4.9 \mathrm{mM}$ in the EtOH alone group. Saline, EtOH alone, or EtOH with the lower concentration of POA caused no discernable pancreatic injury. In contrast, EtOH with the higher concentration of POA induced significant increases of serum amylase (peak at $12 \mathrm{~h}$ ), pancreatic trypsin and myeloperoxidase activity, accompanied by marked histopathological changes that peaked at 24h. All parameters decreased at $48 \mathrm{~h}$. Conclusion: Our results indicate that concomitant administration of EtOH and POA causes acute pancreatitis. Since this novel model may parallel clinical acute alcoholic pancreatitis, it could be a useful tool to study alcoholinduced acute pancreatitis.

\begin{tabular}{ll}
\hline KARGER & (C) 2011 S. Karger AG, Basel and IAP \\
1424-3903/11/0117-0001\$38.00/0 \\
$\begin{array}{l}\text { Fax +4161306 1234 } \\
\text { Eail karger@karger.ch } \\
\text { www.karger.com }\end{array}$ & $\begin{array}{l}\text { Accessible online at: } \\
\text { www.karger.com/pan }\end{array}$
\end{tabular}




\section{AP-B3 \\ Cytokine Imbalance Induces Autoimmune Pancreatitis in Mice}

\section{A. Nishio, K. Uchida, T. Fukui, K. Okazaki \\ Third Department of Internal Medicine, Kansai Medical \\ University, Moriguchi, Japan}

Background: Autoimmune pancreatitis (AIP) is a discrete entity of pancreatitis that is characterized by a steroid-responsive, fibroinflammatory condition that often involves multiple organs. Animal models have been used to study the autoimmune mechanism of AIP. Aim: To investigate the pathogenesis of murine AIP induced by tolllike receptor (TLR) stimulation. Methods: Six-week-old female MRL mice were injected intraperitoneally with polyinosinic polycytidylic acid (poly I:C) or lipopolysaccharide (LPS) at doses of $5 \mathrm{mg} / \mathrm{kg}$ body weight twice weekly for 12 weeks. The mice were killed and the severity of pancreatitis was graded using a histological scoring system. Serum cytokine levels of mice with pancreatitis and mice that were given a single injection of TLR ligands were measured using enzyme-linked immunosorbent assays. The effect of TLR stimulation on the development of pancreatitis was also examined using C57BL/6 interleukin (IL)-10-deficient (KO) mice. Results: Administration of poly I:C accelerated the development of pancreatitis in MRL mice, but LPS did not. Serum levels of IL-10 and IL-12 were significantly elevated in mice with AIP. A single injection of LPS markedly increased serum levels of IL-10, interferon- $\gamma$, tumor necrosis factor- $\alpha$ and IL-12 compared with those of poly I:C-treated mice. Treatment with poly I:C and LPS induced pancreatitis in IL-10 KO mice, but not in wild-type mice. The severity of pancreatitis was greater in mice treated with LPS than in mice treated with poly I:C. Conclusion: Imbalance between proinflammatory and anti-inflammatory cytokines induced by repeated stimulation of innate immunity leads to autoimmunity in the pancreas of mice.

\section{AP-B4 \\ Comparison of the Severity of Acute Pancreatitis Induced by Four or Eight Injections of Caerulein in Swiss Albino Mice}

\author{
T.J. George, A. Kumar, T.S. Roy, P.K. Garg \\ Department of Gastroenterology, All India Institute of \\ Medical Sciences, New Delhi, India
}

Background: Animal models help study the pathophysiological mechanisms of acute pancreatitis (AP). Caerulein induced AP is a common model in mice. Generally, $8-12$ injections of $50 \mu \mathrm{g}$ of caerulein every hour are used to induce AP but there is a wide variability in the dosage and duration of caerulein from 3 to 12 injections. No comparative data are available between low and high dosage of caerulein. Objective: To compare the severity of AP between 4 and 8 , hourly injections of caerulein. Methods: 24 adult male Swiss albino mice were divided into 3 groups of 8 animals each: Group I was given saline injections; group II was given hourly intra-peritoneal injections of caerulein $(50 \mu \mathrm{g} / \mathrm{kg})$ for 4 hours; and group III was given the same treatment for 8 hours. The animals were sacrificed 1 hour after the last injection. Severity of AP was assessed by serum amylase, lung myeloperoxidase, histopathological score, and electron microscopic examination. Results: Both low and high dose caerulein induced necrotizing pancreatitis although the severity of pancreatitis was more in the high dose group. The histopathological scores were: group I $0.25 \pm 0.1$, group II $8.85 \pm 0.5$ and group III $9.82 \pm 0.5$. The amylase levels were high in both low and high dose caerulein groups $(39.69 \pm 3.38 \mathrm{U} / \mathrm{mL}$ vs. $53.75 \pm 2.44 \mathrm{U} / \mathrm{mL} ; \mathrm{p}=\mathrm{NS})$. Lung myeloperoxidase levels were higher in the high dose group (20.65 vs. 2.35, $\mathrm{p}=0.03$ ). Electron microscopic examination showed autophagic vacuoles, apoptosis and necrosis in both the low and high dose groups. Conclusion: Caerulein given for 4 hours induces severe necrotizing pancreatitis sufficient to study the pathophysiological events and facilitates early completion of experiment.

\section{AP-B5 \\ Myocardial Dysfunction in Experimental Acute Pancreatitis}

A.M. Meyer, V.M. Salemi, A.M.M. Coelho, M.S. Kubrusly,

R.A. Patzina, J. Jukemura, M.C.C. Machado,

L.A.C. D'Albuquerque

Department of Gastroenterology and Biochemistry, University of Sao Paulo, Brazil

Background: Evidences suggest that proinflammatory cytokines such as IL-1, TNF $\alpha$, IL-6 and IL- 8 act as mediators of local and systemic manifestations in acute pancreatitis and correlate with the severity of the disease. The mechanisms of myocardial injury in acute pancreatitis are not completely understood. The production in situ into the myocardium of cytokines may lead to acute myocardial damage with functional changes and, eventually, chronic sequelae. Aim: To evaluate the histological and functional changes of the heart in acute pancreatitis and to correlate with the production of cytokines in situ into the myocardium. Methods: Adult Wistar male rats were subjected to experimental acute pancreatitis. Myocardial function was evaluated by using echocardiography, rats were sacrificed for biochemical determination, histochemical and TGF $\alpha$ gene expression study at 2, 4, 12 and $24 \mathrm{~h}$ and 15 days. Results: We observed decrease diastolic and systolic function, decrease in ventricular compliance with fibromuscular lesions and elevation of TGF $\alpha$ mRNA levels demonstrating the occurrence of fibrosis and cellular repair. Conclusion: We conclude that in acute pancreatitis there are function damage and increasing $\mathrm{TGF} \alpha$, probably related to myocardial reparation process. 


\section{AP-B6 \\ Xigris Modulates the Sub-Cellular Expression of Initiator and Effector Caspase in L-arginine Induced Acute Pancreatitis}

\author{
B.I. Babu, A.K. Siriwardena, M. Jeziorska \\ Academic HPB Unit, Manchester Royal Infirmary, \\ University of Manchester, UK
}

Introduction: Xigris (Recombinant human activated protein $\mathrm{C}$ ) is known to modulate apoptosis in acute pancreatitis (AP). Aim: Utilising immunohistochemistry (IHC) and image analysis, we quantified the effect of Xigris on the apoptotic pathway in an L-arginine induced AP model. Method: Under UK Home Office Licence adult Sprague Dawley rats were allocated as follows: Gp 1: control; Gp 2: AP; Gp 3; AP pre-treated with Xigris $(500 \mu \mathrm{g} / \mathrm{kg} \mathrm{bw})$; Gp 4: AP with Xigris 30 minutes after induction of injury. AP was induced by intraperitoneal administration of L-arginine $(300 \mathrm{mg} / 100 \mathrm{~g}$ BW). Animals were sacrificed at 48 hours. IHC and computerised image quantification was performed for Caspase 8, 9, 3. Results: Quantitative increased expression of Caspase 8, 3 and 9 in the AP group compared to the controls was noted. A non-significant decrease was present in both pre and post treatment groups for all three caspases (Kruskal Wallis test $\mathrm{P}=0.2558$ ). Increased expression of Caspase 8 was noted in all the groups compared to the other caspases ( $\mathrm{P}=0.1631$, Kruskal-Wallis). Caspase 9 was the least expressed. Conclusion: As apoptotic cascade proceeds, a quantitative amplification of effector caspase 3 compared to initiator caspase 8 is expected. This was not demonstrated in our study raising the possibility of Xigris acting prior to or at Caspase 3. No demonstrable increase was noted in the expression of caspase 9 as apoptosis was triggered by an extrinsic mechanism. This study for the first time provides insight in to the sub cellular basis for the potential efficacy of Xigris in AP.

\section{AP-B7 \\ Mitochondrial Targeted Therapy to Reduce Multiple Organ Dysfunction in Acute Pancreatitis}

\author{
A. Hickey, A. Dare, A.R.J. Phillips \\ Maurice Wilkins Centre for Molecular Biodiscovery, \\ University of Auckland, New Zealand
}

Background: Severe acute pancreatitis (AP) is associated with development of multiple organ dysfunction syndrome (MODS). Recently, mitochondrial dysfunction (MD) has been proposed as a key early cellular event in the development of MODS in critical illness. Treatment targeted to protect mitochondria may prevent or reduce early MD, reducing morbidity and mortality in MODS. This study aimed to evaluate the efficacy of a novel specific mitochondrial treatment (MT01) in experimental AP. Methods: AP was induced in male Wistar rats randomized into (i) saline control, (ii) AP control and (iii) AP with MT01 treatment groups. Animals were euthanased six hours after AP induction and their tissues and blood collected for analysis. The primary endpoint was mitochondrial respiration (pmol/ $\mathrm{O} 2 / \mathrm{mg}$ protein/second), determined using high resolution respirometry (Oroboros Oxygraph, Graz, Austria), in tissue homogenates. Secondary endpoints included lipid peroxidation and severity of tissue injury by histology. Results: Mitochondrial respiration was significantly depressed in the pancreas, jejunum and lung (but not the kidney, heart or liver) during untreated AP compared to saline controls. In the jejunum and lung this occurred in the absence of significant histological changes. Treatment with MT01 decreased MD of the jejunum, and lung, and increased mitochondrial respiration capacity in the kidney during AP. The treatment was also associated with a decrease in serum lipid peroxidation markers. Conclusion: MD occurs in the pancreas, jejunum and lung early in the course of AP and before histological evidence of tissue injury. In extra-pancreatic organs, MD can be reduced by MT01. These findings highlight a new therapeutic target at the organelle level for AP induced MODS.

\section{AP-B8 \\ Protective Effect of Caffeine in Murine Acute Pancreatitis}

W. Huang, R. Mukherjee, M.C. Cane, V. Elliott, D.M. Booth, E. McLaughlin, O.H. Petersen, A. Tepikin, D.N. Criddle, R. Sutton

Liverpool NIHR Pancreas Biomedical Research Unit, Royal Liverpool University Hospital, Liverpool, UK; Physiological Laboratory, University of Liverpool, Liverpool, UK; Cardiff School of Biosciences, Cardiff University, Cardiff, UK

Background and Aims: How coffee consumption reduces the risk of alcoholic pancreatitis is unclear. We have investigated the effects of caffeine on pancreatic acinar cells in vitro and acute pancreatitis models in vivo. Methods: Confocal microscopy was used to assess the effect of caffeine on cytosolic calcium $([\mathrm{Ca} 2+] \mathrm{C})$ in pancreatic acinar cells stimulated by $10 \mathrm{nM} \mathrm{CCK}-8$ or $500 \mu \mathrm{M}$ taurolithocholate-sulphate (TLC-S). CD1 mice received caerulein $(50 \mu \mathrm{g} / \mathrm{kg} / \mathrm{h}$ ip hourly $\mathrm{x} 7$ or $\mathrm{x} 12$ ), or retrograde ductal infusion of TLC-S. Caffeine at $1,5,10$ or $25 \mathrm{mg} / \mathrm{kg} / \mathrm{h} \times 7$ was given from $3 \mathrm{~h}$ after the first caerulein injection, or $1 \mathrm{~h}$ after TLC-S infusion, with serum concentrations determined at various time points. Severity of pancreatitis was assessed by standard biomarkers and histopathology. Results: In vitro, $10 \mathrm{mM}$ caffeine fully inhibited the $[\mathrm{Ca} 2+] \mathrm{C}$ plateau induced by CCK-8 or TLC-S in isolated cells. In vivo, Caffeine ameliorated the severity of pancreatitis in both models. This effect was dose-dependent with $25 \mathrm{mg} / \mathrm{kg} / \mathrm{h}$ x 7 ( $1 \mathrm{mM}$ peak serum concentration) improving all parameters of pancreatitis remarkably except lung injury. Conclusion: Caffeine is protective in experimental pancreatitis induced by either caerulein or TLC-S. The effect of caffeine may occur in part via inhibition of pathological $[\mathrm{Ca} 2+] \mathrm{C}$ elevation. 
Acute Pancreatitis - Clinical

\section{AP-C1 \\ Outcomes and Complications of EUS Guided Drainage of Pancreatic Pseudocyst Utilizing CRE Dilation, Endoprosthesis and Naso- cystic Drains: A Large Single Tertiary Referral Center Experience}

\section{S.R. Mishra, R. Puri, R. Sud}

Department of Digestive and Hepatobiliary Sciences, Medanta The Medicity, Sector 38, Gurgaon, Haryana, India

Introduction: Endoscopic ultrasound (EUS)-guided transmural drainage for pancreatic pseudocysts has gained important momentum in the management of pancreatic pseudocysts. We evaluated the outcome and complications of EUS-guided pancreatic pseudocyst drainage (EUS-PPD). Methods: Between August 2007 and January 2010 , EUS-PPD was attempted in 40 patients with pancreatic pseudocyst which was (i) symptomatic; (ii) resistant to conservative treatment; (iii) the cystic wall was in contact with the gastric or duodenal wall on EUS and (iv) no bulge seen in upper gastrointestinal endoscopy. CRE (Controlled Radial Expansion) wire guided balloon (up to 12 to $15 \mathrm{~mm}$ ) dilation of the puncture tract followed by a 10 French $5 \mathrm{~cm}$ double pigtail stent placed in cyst cavity and a 7-Fr. pigtail-type nasobiliary drainage tube was inserted into the cyst. The early and late outcomes, and complications of EUS-PPD were retrospectively analyzed. Results: Placement of double pigtail stent and naso-cystic drain was successful in $100 \%$ of patients. The pseudocyst was completely resolved after the placement in 39 patients, one patient required surgical operation in early period. Discharge of the cystic fluid was stopped and the naso-cystic tube was removed in 39 patients after a median duration of 13 days. Thereafter, the double pigtail stent was removed in all cases after a median duration of 10 weeks. Recurrence of the pseudocyst occurred in 2 patients during a median follow-up period of 48 months. All 31 patients without cystic infection were successfully treated by EUS-PPD. On the other hand, 2 out of 8 patients $(25 \%)$ with pseudocyst infection had recurrence of pseudocyst and one patient underwent a second session of EUS guided drainage while another patient was subjected to surgical resection. No serious complications related to the procedure occur in any patients during and after the procedure. Conclusion: EUS-PPD is a safe and effective treatment for pancreatic pseudocyst. Infected pseudocysts are often difficult to treat by only EUS-PPD, thus additional treatments should be considered at an early stage.

\section{AP-C2 \\ Evaluation of the Safety of Twenty-four Hour Infusion of Human Recombinant Activated Protein C (XIGRIS) Early in Severe Acute Pancreatitis: Initial Report of the XIGAP-1 Study}

\author{
A.K. Siriwardena, C.J. Miranda, J.M. Mason, B.I. Babu, \\ J. Eddleston, M.J. France
}

The Manchester HepatoPancreatoBiliary Centre, Manchester Royal Infirmary, UK

Introduction: Severe acute pancreatitis (SAP) remains a condition with a high morbidity and risk of death with no specific pharmacologic treatment. Human recombinant activated protein C (xigris) modifies coagulation and inflammatory cascades and reduced mortality in a large randomized trial in sepsis (PROWESS). As xigris can cause haemorrhage, SAP was a specific exclusion criterion in PROWESS. This study reports the initial clinical experience with the use of xigris for the early treatment of SAP. Methods: SAP was defined as CT-confirmed AP within 96 hours of severe pain and 72 hours of admission; Acute Physiology And Chronic Health Evaluation (APACHE II) score $>9$, plus a minimum of two of four systemic inflammatory response (SIRS) criteria measured on two occasions separated by at least 24 hours plus a Marshall organ dysfunction score (MODS) of $\geq 2$ for any system. Following regional ethics committee approval, XIGAP-1 opened to recruitment in November 2009. Of 71 screened AP admissions to a tertiary hepatobiliary unit, 11 were eligible and 6 enrolled (4 female, gallstone 3, alcohol 3). Median (range) APACHE II score prior to infusion of xigris was 18 (12-22). Median in-patient stay was 21 (9-58) days and median Critical Care Occupancy 17 (1-24) days. The focus of early experience was on haemorrhage. Results: Infusion of xigris was not associated with either pancreatic, gastrointestinal or remote bleeding. Median MODS score prior to infusion was $5(2-7)$ and post-xigris $1.5(0-4) .30$ day mortality was 1. Conclusion: Early administration of xigris to a carefully-defined population of SAP is not associated with an increased incidence of haemorrhage. Further experience is required before an assessment can be made as to whether there should be an international multi-centre randomized trial of xigris in SAP.

\section{AP-C3}

Minimally Invasive Pancreatic Necrosectomy - How to Choose the Best Approach?

\section{A. Nagpal, H. Soni, S. Haribhakti \\ Department of Surgical Gastroenterology and Advanced Laparoscopic Surgery, Haribhakti Surgical Hospital, Ahmedabad, India}

Introduction: Several minimally invasive approaches to pancreas have been described, such as laparoscopic, retroperitoneoscopic and endoscopic for necrotizing pancreatitis. Variations in the extent and location of necrosis and the condition of the patient determines a tailored approach to the treatment of pancreatic necrosis. Aim: To 
describe our experience with the use of laparoscopic and retroperitoneoscopic methods for the debidement of the necrosis and to determine the correct approach based on local and systemic factors. Methods: 8 patients were treated by various minimally invasive techniques for infected pancreatic necrosis. Evidence of infection was the presence of gas in retroperitoneum ( 3 cases) and FNA positive for infection ( 2 cases). Entire decision-making process was tailormade for each case individually as per the patient's history, disease condition, disease location and morphology on CT scan. Results: Laparoscopic transperitoneal necrosectomy was chosen in 3 patients who had primarily single collection and in patient who required simultaneous cholecystectomy. Retroperitoneoscopic (RP) necrosectomy was attempted in 5 patients who had disease involving mainly the distal pancreas and the left side of the abdomen. No patient needed conversion to open surgery. However, two patients of the RP necrosectomy group required repeat RP necrosectomy and then open necrosectomy later on. Mean operating time was approximately 100 minutes with mean postoperative hospital stay of 35.66 days. Three patients developed controlled pancreatic fistula after RP necrosectomy, which healed conservatively on follow-up (at 32, 17 and 47 days). Mortality occurred in 2 patients due to Intra- abdominal bleeding and malignant hyperpyrexia. Conclusion: Minimally invasive pancreatic necrosectomy is a feasible technique with acceptable results in carefully selected patients by experienced surgeons. Laparoscopic approach is feasible in patients who have unilocular, retrogastric fluid collections with the additional advantage of allowing simultaneous cholecystectomy in indicated patients. Retroperitoneal approach may be selected in patients with left-sided pancreatic necrosis with predominantly semisolid debris.

\section{AP-C4 \\ Predictive Factors and Outcome of Colonic Fistulae in Severe Acute Necrotising Pancreatitis}

M. Gupta, S. Dhar, B.V. Dinesh, S. Lalwani, P. Singla, A. Yadav, V. Varma, N. Mehta, V. Kumaran, S. Nundy

Department of Surgical Gastroenterology and Liver Transplantation, Sir Ganga Ram Hospital, New Delhi, India

Background: Colonic involvement in acute necrotising pancreatitis accounts for $6-40 \%$ of cases. Incidence of colonic fistula in various series varies from $2-10 \%$. Resection of involved diseased colon with diverting stoma is the treatment of choice as reported in the literature. Aim: To study factors predicting colonic fistulae in severe acute necrotising pancreatitis. Methods: We performed a retrospective review of 131 patients who underwent pancreatic necrosectomy for acute necrotising pancreatitis from 1996 to 2010. We identified patients who developed colonic fistula prior to pancreatic necrosectomy, at the time of necrosectomy and post necrosectomy. CT scan was used to grade the severity of pancreatitis. Results: Fourteen patients were identified to have colonic fistula. Colonic fistula diagnosed after necrosectomy (8), prior to necrosectomy (3) and at the time of necrosectomy (3). Pancreatic necrosectomy was performed after mean interval of 41 days. Diverting ileostomy was performed in all (14) patients. Four patients required colectomy (formal colectomy 2 and segmental colectomy 2 ) and fis- tula closure occurred in 5 patients with ileostomy alone. Mean duration of fistula closure was 47 days. Preoperative modified CT severity index $(p$ value $=0.001)$ and operative findings $(p$ value $=0.00)$ were found to be the significant factors associated with fistula formation. Operative mortality was $29 \%(4 / 14)$ in patients with fistula whereas in patients without fistula mortality was $22 \%$ (26/117). Conclusions: Preoperative modified CT severity index and extensive pericolitis at the time of laparotomy were predictive of colonic fistula in severe acute necrotising pancreatitis and diverting ileostomy (perhaps during necrosectomy) in such cases might be an effective preempting manoeuvre.

\section{AP-C5 \\ Dynamic Nature of Organ Failure in Acute Pancreatitis and Its Outcome}

\section{R.B. Thandassery, R.K. Kochhar, T.D. Yadav, S.M. Chowdry, U. Dutta, K. Singh}

Departments of Gastroenterology and General Surgery, Postgraduate Institute of Medical Education and Research, Chandigarh, India

Background: Organ failure (OF) in 1st week of acute pancreatitis (AP) can be transient (TOF, duration of organ failure lasting $<48$ hours) or persistent (POF, duration of organ failure lasting $>48$ hours). Aim: To compare the clinical course and outcome of patients with TOF and POF in 1st week of admission among those with AP. Methods: Consecutive patients with AP (January 2009-June 2010) were evaluated for OF, its severity and dynamics during 1st week of admission. Modified multiple organ failure score (MOFS) was used to identify and grade severity of OF. Statistical analysis was done with SPSS version 16. Results: Of the 81 patients, mean age 40.1 years (range 13 to 84 ), 55 males; $21(26 \%)$ patients had no OF, $26(32 \%)$ had TOF and 34(42\%) had POF in the 1st week. Between POF and TOF; former had longer hospital stay $(20.6 \pm 2.2$ and $13.1 \pm 2.2$ days, $\mathrm{p}=0.055)$, longer ICU stay $(12.32 \pm 2.1$ and $0.35 \pm 0.27$ days, $\mathrm{p}=0.000)$, more infections $(23 ; 67.6 \%$ and $4 ; 15.3 \%, \mathrm{p}=0.000)$, more infected necrosis $(9 ; 90 \%$ and $1 ; 10 \%, p=0.02)$, more local complications $(32 ; 94 \%$ and $20 ; 77 \%, p=0.142)$, more requirement for percutaneous drain placement $(16 ; 47 \%$ and $5 ; 26 \%, \mathrm{p}=0.042)$, more requirement for surgery $(10 ; 29.4 \%$ and $2 ; 7.6 \% \mathrm{p}=0.01)$, more requirement for ventilatory support $(0 \%$ and $12 ; 35.3 \% \mathrm{p}=0.000)$ and more mortality $(0 \%$ and $13 ; 38 \% \mathrm{p}=0.001)$. By logistic regression analysis, APACHE II score (at 48 hours), worsening of MOFS and infected necrosis were independent predictors of mortality. Conclusion: Sub classification of patients with AP into TOF and POF predicts clinical course and outcome. Patients with POF have poor outcome and prolonged course as compared to those with TOF. 


\section{AP-C6 \\ Hypotension in 1st Week of Acute Pancreatitis Predisposes to Development of Infected Pancreatic Necrosis}

\section{R.B. Thandassery, R.K. Kochhar, T.D. Yadav, S.M. Chowdry,} U. Dutta, K. Singh

Departments of Gastroenterology and General Surgery, Postgraduate Institute of Medical Education and

Research, Chandigarh, India

Background: Hypotension and intestinal mucosal ischemia leads to bacterial translocation from lumen into systemic circulation. Aim: To correlate the occurrence of hypotension (CVS failure), renal failure, respiratory failure CNS failure and coagulopathy in the 1st week of acute pancreatitis (AP) with subsequent development of infected necrosis (IN). Methods: Consecutive patients with AP (January 2009-June 2010) were evaluated for organ failure (OF) and its severity in first week of admission. Modified multiple organ failure (Modified Bernard score) score was used to identify and grade severity of OF. The occurrence of IN (isolation of bacteria in necrosectomy specimen or CT guided FNAC of pancreatic necrosis) was compared between groups with and without OF. Statistical analysis performed with SPSS version 16. Results: Of the 81 patients, mean age 40.1 years (range 13 to 84 ), 55 males; 60(74\%) patients had OF. $13(16 \%)$ patients (total 15 isolates, all of enteric origin) had IN (67\% Gram negative). Occurrence of IN was not significantly different between patients with OF (18.3\%) and without OF $(14.3 \%), p=0.48$. However IN occurred in $28.6 \%$ patients with and $11.7 \%$ patients without hypotension respectively, $p=0.04$. Rest of the organ systems analysed did not show any significant difference in occurrence of infected or sterile necrosis. IN was present in $13.3 \%$ patients without and $19.4 \%$ with respiratory failure, $p=0.32$. Renal failure, coagulation failure and CNS failure also did not differ in patients with or without $\mathrm{IN} ; \mathrm{p}=0.32,0.14$ and 0.41 respectively. Conclusion: Hypotension in the 1st week of AP is a risk factor for development of IN.

\section{AP-C7 \\ Infections in Acute Pancreatitis and Its Impact on Outcome}

R.B. Thandassery, R.K. Kochhar, T.D. Yadav, P. Dixit, U. Dutta, K. Singh

Departments of Gastroenterology and General Surgery, Postgraduate Institute of Medical Education and Research, Chandigarh, India

Background: Infected necrosis (IN) signifies a poor outcome and the bacteriology of IN needs to be identified for appropriate antibiotic therapy. Aim: To study the bacteriology of IN and its outcome in patients with acute pancreatitis (AP). Methods: All consecutive patients with AP (January 2009-June 2010) were evaluated for occurrence of infections and IN. Statistical analysis was carried with SPSS version 16. Results: Of the 81 patients, mean age 40.1 (range 13 to 84 ) 55 males; 33 (40.7\%) had infection, with 98 bacterial isolates. The source of which was blood (33), percutaneous drain (23), CT guided fine needle aspiration cytology (CT - FNAC, 6), operative specimen (15) and others (21). 62 (70.5\%) of isolates were Gram-negative. The duration of hospital stay for patients with Gram positive infections ( $32.9 \pm 13.5$ days) was significantly more than those with Gram negative infections (20.2 \pm 12.4 days $), p=0.01$. $16 \%$ of patients had infected necrosis (isolation of bacteria in CT-FNAC of infected necrosis or necrosectomy specimen). $62 \%$ of IN was monomicrobial and $38 \%$ polymicrobial. The most common organism isolated from infected necrosis was Enterococcus fecalis $(n=7,34 \%)$ followed by Escherichia coli $(n=5,23 \%)$. The antibiotic sensitivity pattern showed that most bacteria were sensitive to third generation cephalosporins (ceftriaxone and ceftazidime) and quinolones. The mortality was more in patients with infected necrosis $(76.9 \%)$ as compared to non infected necrosis $(8.8 \%), p=0.001$. Conclusion: Most of the infections in AP are due to Gram negative organisms. Gram positive infections are associated with longer hospital stay. Infected pancreatic necrosis is associated with high mortality.

\section{AP-C8 \\ Predictors of Mortality in Severe Acute Pancreatitis}

\author{
M.K. Hinduja, T.D. Yadav, R.K. Kochhar, A. Agrawal, \\ R. Singh
}

Departments of General Surgery and Gastroenterology, Postgraduate Institute of Medical Education and Research, Chandigarh, India

Background: Mortality in patients with severe acute pancreatitis (SAP) is associated various clinical, investigational and treatment factors. Aims: To determine predictors of mortality in SAP prospectively in a tertiary care hospital in India. Methods: 66 patients of SAP were studied prospectively between Jan, 2009 and June, 2010 for clinical features, lab parameters, Ranson's and APACHE II scores and CT severity index. All patients were managed with prophylactic antibiotics, nasojejunal/parenteral nutrition and supportive care. Necrosectomy was done for persistent organ failure, infected necrosis and clinical deterioration. Factors affecting mortality were analyzed. Results: Of the 66 patients (45 males, age 13-84 years, etiology: 31 alcohol, 24 gallstones and 9 others), 21 underwent percutaneous catheter drainage and 16 underwent necrosectomy. 16 patients died, of whom 12 had been operated. On univariate analysis factors associated with mortality were obesity $(\mathrm{p}=0.001)$, number of comorbidites $(\mathrm{p}=0.001)$, duration of pancreatitis $(\mathrm{p}=0.001)$, presence of low $\mathrm{pH}(\mathrm{p}=0.001)$ and base deficit $(\mathrm{p}=0.001), \mathrm{CT}$ severity index $>7$ $(p=0.001)$, extent of necrosis at admission $(p=0.001)$, Ranson's score at $48 \mathrm{~h}(\mathrm{p}=0.001)$, APACHE II score at admission $(\mathrm{p}=0.001)$, number of organ failures $(p=0.001)$, requirement of surgery $(p=0.001)$, and post-operative enteric fistula $(\mathrm{p}=0.001)$. On multivariate analysis, base deficit, serum bilirubin and need for surgery were independent predictors of mortality. Conclusion: Factors at admission correlating with mortality were comorbidities, APACHE II and CTSI scores, multiorgan failure, high serum bilirubin and high base deficit. Patients managed conservatively survived more often than those operated. 


\section{AP-C9}

\section{Early vs Late Mortality in Acute Pancreatitis: Analysis of 180 Patients}

\author{
M.K. Hinduja, T.D. Yadav, R.K. Kochhar, A. Agrawal, \\ R. Singh \\ Departments of General Surgery and Gastroenterology, \\ Postgraduate Institute of Medical Education and \\ Research, Chandigarh, India
}

Background: Despite all efforts to make an early diagnosis and start timely treatment of acute pancreatitis (AP), mortality is still high in patients with severe acute pancreatitis. Aim: To compare factors affecting early ( $<2$ weeks) versus late ( $\geq 2$ weeks) mortality in patients with AP. Methods: All patients dying of AP between 2004 and 2008 were retrospectively analyzed for demographics, duration of AP, lab parameters, APACHE II score, pancreatic necrosis and need for surgery/ postoperative complications. Results: In 180 patients (12-80 yr, 122 males), etiology of AP was alcohol (52.8\%), gallstones (31\%), trauma $(10 \%)$ and others (5\%). 33\% of deaths occurred early and $67 \%$ late. The primary cause of death was organ failure in 102 patients and sepsis in 73 patients. Comorbidities $(p=0.001)$, elevated serum creatinine $(p=0.001)$, elevated blood glucose $(p=0.003)$, low $\mathrm{pH}$ $(p=0.001)$ and high base deficit at admission were significantly associated with early death. Total number of organ failures at admission and presence of any organ failure were associated with early death $(p=0.001)$. Only $16.6 \%$ of patients had been operated in the early death group as compared to $64.2 \%$ in the late death group. Multiorgan failure was the commonest cause of death in early death group and sepsis in the late death group. Of the 102 deaths due to sepsis 19 (18.6\%) were early deaths and $83(81.3 \%)$ were late deaths. Of the 73 patients who died of organ failure $40(59 \%)$ had early death and 32 (41\%) had late death. On multivariate analysis, the only predictor of early death was the number of organ failures at admission. Conclusions: Number of organ failures at admission predicts early mortality in patients with AP. Sepsis is responsible for most late deaths.

\section{AP-C10 \\ Can the Time Course of SIRS Predict Future Organ Failure in Acute Pancreatitis?}

\author{
A. Kumar, S.T. Chari, S.S. Vege \\ Division of Gastroenterology and Hepatology, Mayo Clinic, \\ Rochester, Minnesota, USA
}

Background/Aims: Systemic Inflammatory Response Syndrome (SIRS) (possible score 0-4) is an easily measured bedside tool. In patients with acute pancreatitis (AP) our aim was to validate the role of SIRS on day of diagnosis of AP (day 1) and of persistent SIRS in predicting the severity. Further we have studied the time course of SIRS during hospitalization to see if it has any prognostic significance. Method: We prospectively identified all patients admitted to the Mayo Clinic on day 1 of AP from June 2004 to August 2005. We analyzed the association between positive SIRS (Score $>/=2$ ) on day1, persistent SIRS on day 3 and the adverse outcomes of AP. All patients with positive SIRS on day 1 were further followed up for persistent SIRS and organ failure for 14 days or till discharge, whichever came first. We correlated the time course of SIRS with development of organ failure. Results: Out of the 117 patients, 54 (46.2\%) had positive SIRS on day 1 . Out of these $35(66 \%)$ had persistent SIRS on day 3. Both these groups had a higher incidence of all adverse outcomes with a high sensitivity (70-100\%) and negative predictive value $(80-100 \%)$. Persistent SIRS on day 3 was also fairly specific (71-81\%) for various complications of AP. The majority of OF had its onset in the first 72 hours $(77.7 \%)$. The cumulative number of days with positive SIRS has the potential of predicting future OF in those with late onset. Conclusion: SIRS and persistent SIRS are associated with all adverse outcomes of AP with a high sensitivity and negative predictive value. Persistent SIRS at day 3 adds specificity to this association. Cumulative number of SIRS positive days has the potential for predicting future new onset organ failure and needs to be studied prospectively.

\section{AP-C11 \\ A Randomised Control Study on Effect of Octreotide in Management of Acute Pancreatitis with Varying Severities}

\author{
S.P. Rath, A.P. Basu, D.P. Patra, S.P. De
}

Department of General Surgery, Institute of Postgraduate Medical Education and Research, and Seth Sukhlal Karnani Memorial Hospital, Kolkata, West Bengal, India

Background: Acute pancreatitis is an acute inflammatory process of the pancreas with variable involvement of other regional tissues or organ systems. Theories on pathogenesis of acute pancreatitis suggest that auto digestion of the gland and peripancreatic tissues by activated digestive enzymes is a key component. Octreotide is a potent inhibitor of pancreatic secretion, but the benefits of octreotide in acute pancreatitis in humans is still inconclusive. Aim: To study the effect of octreotide in acute pancreatitis with different severities. Method: 284 patients were assigned into two groups randomly, the study group comprising of 138 patients received inj octreotide $100 \mu \mathrm{g}$ S.C. $8 \mathrm{hrly}$. The other aspects of the treatment protocol were similar in both groups. Severity of the disease was evaluated initially according to Ranson criteria, APACHE II SCORING, CRP values and Balthazar grading. Both the groups were observed prospectively and outcome of treatment was evaluated. Results: The results were analysed after dividing both the study and control group into 5 groups each according to increased severities. The complication rate was $10 \%$ in group 1 and $28 \%$ in group 2 according to APACHE II among the study group, whereas it was $23 \%$ and $46 \%$ in the control group respectively. The values in patients stratified according to other criteria's were also comparable. The results in cases of severe pancreatitis were poor, complication rates varying from $56 \%$ to $100 \%$ in the $3 \mathrm{rd}$ and 5th group according to Ranson criteria among the study group and $62 \%$ to $100 \%$ respectively among the control group. Mortality of the study and control group also did not show any significant changes. Conclusion: Inhibition of secretory function in the early course of the disease especially in mild and moderate cases of pancreatitis has been shown to improve final outcome. As in case of acute pancreatitis it is very difficult to estimate the severity and disease progress at the 
very onset, and as the majority of cases are mild to moderate in severity, it will be beneficial to start with octreotide in any treatment protocol along with supportive resuscitative measures.

\section{AP-C12 \\ EUS Guided Single Step Complete Aspiration of Peripancreatic Fluid Collections and Pseudocysts Following Acute Pancreatitis: A Pilot Study}

\author{
V. Dhir, P. Matthew, M. Bapat, S. Bhandari, A. Maydeo \\ Institute of Advanced Endoscopy, Mumbai, India
}

Background: EUS guided pseudocyst drainage is now an established treatment modality. However there are reports of bleeding or abscess formation due to cautery use and stent placement. Aim: To evaluate the safety and efficacy of single step EUS guided total aspiration of peri-pancreatic fluid collections/pseudocyst in patients with acute pancreatitis. Methods: Between Jan 2009 to June 2010, 2 patients with symptomatic peri-pancreatic fluid collection and 9 patients with pseudocysts were recruited in the study. Each patient underwent EUS guided total aspiration with a 19 gauge needle with all aseptic precautions. All patients were re-assessed for recurrence of fluid collection after one week, and those with recurrent fluid were considered for other treatments. Results: All patients presented between 3-9 weeks following the acute episode. A median of $700 \mathrm{cc}$ fluid (100 cc-1.3 litres) was removed over $40 \mathrm{mins}$ (20-60 mins). Mean size of PPF was $6.5 \pm 5.5 \mathrm{cms}$. Both patients with PPF did not respond to single sitting of aspiration (one could not be drained due to deep location and second refilled within 4 days). 7/9 patients with pseudocyst had associated debris within. Mean size of pseudocyst was $7 \pm 5.5 \mathrm{cms}$. $7 / 9$ pseudocyst patients responded to single sitting of aspiration. 1 of 9 pseudocyst patients developed infection $(11.1 \%)$ and required surgery. Success of single sitting EUS aspiration was $77.7 \%$ in pseudocysts. Conclusion: EUS guided aspiration as the primary treatment modality has good success rate with acceptable incidence of infection/bleeding in patients with pseudocysts following acute pancreatitis. The results in PFC are not so encouraging.

\section{AP-C13 \\ Systemic Inflammatory Response Syndrome (SIRS) Score at Admission Predicts the Development of Primary Intra-abdominal Infection in Patients with Acute Pancreatitis}

\section{R. Talukdar, S.S. Vege, M. Clemens \\ Miles and Shirley Fiterman Center for Digestive Diseases, Department of Internal Medicine, Mayo Clinic College of Medicine, Rochester, MN, USA}

Background and Aim: Presence of organ failure in acute pancreatitis (AP) is known to be associated with infected necrosis and high mortality. However, there are no known early predictors of intra- abdominal infection in AP. The aim of this study is to assess the capability of simple baseline parameters to predict intra-abdominal infection. Methods: We prospectively studied 274 consecutive patients with acute pancreatitis (AP) directly admitted to Mayo Clinic hospitals over a two year period. We identified the patients who had microbiologically confirmed infections of pancreatic necrosis and peripancreatic fluid collections; and defined primary intra-abdominal infection as any infection that developed prior to any abdominal intervention. We recorded admission hematocrit, BMI, serum BUN/creatinine and SIRS score; and used univariate and multivariate conditional logistic regression analysis to assess if these parameters could predict development of intra-abdominal infections. We expressed the results as odds ratio (OR) $[95 \%$ confidence intervals (CI)] and considered a 'p' value of $<0.05$ as statistically significant. Results: Overall, 44 $(16.1 \%)$ patients had infections. $26(9.5 \%)$ had intra-abdominal infection, of whom $20(79.9 \%)$ had primary intra-abdominal infection. Among patients with primary intra-abdominal infection, 8 (40\%) had gram positive, $1(5 \%)$ gram negative and $11(55 \%)$ mixed bacterial infection; while $5(25 \%)$ had additional fungal infection. On univariate analysis, BUN $>25 \mathrm{mg} / \mathrm{dL}$ and SIRS $>2$ were found to significantly predict primary intra-abdominal infection with OR $(95 \% \mathrm{CI})$ of $2.68(0.96-6.96)$ and $3.59(1.42-9.51)$ [2-tailed $p=0.048$ and 0.007 respectively]. On multivariate analysis, only SIRS $>2$ was found to be significantly predictive with an adjusted OR (95\% CI) of 3.13 $(1.21-8.49)[\mathrm{p}=0.018]$. The other study parameters, namely hematocrit, BMI and serum creatinine, did not predict the development of primary intra-abdominal infection. Conclusions: SIRS is a simple and inexpensive severity assessment tool that can predict the development of primary intra-abdominal infection in AP early in the disease course.

\section{AP-C14 \\ Endoscopic Ultrasound During Acute Pancreatitis}

V. Kotwal, R. Talukdar, M.J. Levy, S.S. Vege

Miles and Shirley Fiterman Center for Digestive Diseases, Department of Internal Medicine, Mayo Clinic College of Medicine, Rochester, MN, USA

Background and Aims: There is very limited literature on the role of endoscopic ultrasound (EUS) during the episode of acute pancreatitis (AP). Our aims were to study indications for requesting EUS during AP, findings on EUS, correlation of findings with outcomes in AP and their impact on management. Methods: Patients admitted to Mayo Clinic hospitals for AP between July 2004 to August 2009, and had EUS done during the same admission were identified, charts were reviewed and following information was extracted: Indications for EUS, findings on EUS (visualization of entire pancreas, hypoechogenicity, hyperechogenicity, peripancreatic fluid) and patient outcomes (duration of hospital stay, interventions and ICU transfer). Statistical analysis was done using chi square test. Results: There were total 22 patients ( 8 males, 14 females) with median age $50.5 \mathrm{yrs}$ (IQR 40.75-66). Indications for doing EUS were to confirm bile duct stones $(n=6)$, to look for tumor $(n=5)$, idiopathic pancreatitis $(n=4)$, and in patients with contraindication to CT scan or when findings on CT were doubtful for pancreatitis $(n=3)$. In 2 patients, EUS was done for 
drainage of peripancreatic fluid collection, in one to guide ERCP for pancreatic duct drainage and in another as liver enzymes increased after ERCP. EUS showed normal pancreas in 4 patients (18\%). Most common findings on EUS were peripancreatic fluid in 10 patients $(45 \%)$, and hypoechogenicity in 8 patients $(36 \%)$. Entire pancreas could not be visualized in 5 patients $(22 \%)$ and 2 patients had hyperechogenicity (9\%). None of the above findings on EUS correlated with any of the patient outcomes (hospital stay, intervention and ICU transfer). When compared to CT, out of three patients with pancreatic necrosis on $\mathrm{CT}$, one patient had hypoechogenicity, and the pancreas was not well visualized in the other two. Thus, EUS could not differentiate between interstitial and necrotizing pancreatitis. However EUS correlated well with $\mathrm{CT}$ in all patients with peripancreatic inflammation/fluid and in 2 patients peripancreatic fluid was seen only on EUS but not on CT. The main utility of EUS was that it prevented diagnostic ERCP in 5/6 patients with suspected biliary pancreatitis. EUS detected main duct IPMN in $1 / 5$ patients with suspected tumor and GB sludge in 1/4 patients with idiopathic pancreatitis. EUS could be done safely in 2 patients with contraindication to CT; one had renal failure and could not be given iv contrast and the other was a pregnant female in her first trimester. Conclusion: The most common indications for EUS in AP were suspected biliary pancreatitis or to rule out a tumor. EUS showed normal pancreas in some patients and there was a wide variation in findings in others. EUS did have a role in preventing diagnostic ERCPs, finding the etiology of AP when other imaging modalities had failed and in patients with contraindication to CT scan. However EUS could not differentiate between interstitial and necrotizing pancreatitis and the findings did not have any prognostic significance.

\section{AP-C15 \\ All Patients with Acute Pancreatitis and Persistent Organ Failure Do Not Require Organ Failure Specific Interventions}

\section{R. Talukdar, S.S. Vege, M. Clemens \\ Miles and Shirley Fiterman Center for Digestive Diseases, Department of Internal Medicine, Mayo Clinic College of Medicine, Rochester, MN, USA}

Background and aim: Patients with persistent organ failure (OF) ( $>48 \mathrm{hrs}$ ) in acute pancreatitis (AP) is associated with high morbidity and mortality. All guidelines recommend that these patients be treated in the ICU with OF-specific interventions. The aim of this study was to evaluate if OF-specific intervention was necessary for all patients with AP and persistent OF. Methods: We studied 32 $(11.7 \%)$ patients with persistent OF from a prospective database of 274 consecutive patients admitted with AP over 2 years. We defined OF according to the 1992 Atlanta criteria; and OF-specific interventions as hemofiltration/dialysis for renal failure, BiPAP/CPAP/ mechanical ventilation for respiratory failure, and pressor support/ intra-aortic balloon pump for circulatory failure. Non-OF-specific measures included fluid and oxygen therapy for renal, circulatory and respiratory failures respectively. Outcome variables were OF-specific intervention and ICU care requirement for different OFs. Results: Of the $32(11.7 \%)$ patients with persistent OF, 17 (53.1\%) developed OF during hospital stay and $15(46.9 \%)$ at presentation. Nine patients
(28.1\%) had single OF, 17 (53.1\%) had two OF and 6 (18.8\%) had three OF. $26(81.3 \%)$ had respiratory failure, $24(75 \%)$ patients had renal failure, and $13(40.6 \%)$ had circulatory failure. All patients with respiratory and circulatory failure required OF-specific treatment. Among the patients with renal failure, 15/24 (62.5\%) could be managed with intravenous fluid therapy only. Overall, 5/32 (15.6\%) patients did not require any OF-specific interventions, and all five had only renal failure. Moreover, 3/32 (9.4\%) patients with only renal failure did not require ICU care. Conclusion: This study shows that nearly $16 \%$ of patients with AP with persistent OF did not require any OF-specific intervention and nearly $10 \%$ of such patients could be treated in the wards. All patients who could be treated in the ward and who did not require OF-specific intervention and had only renal failure.

\section{AP-C16 \\ Pediatric Pancreatitis in a South Indian Cohort}

M. Geetha, M. Saumya, V. Balakrishnan

Department of Gastroenterology, Amrita Institute of Medical Sciences, Cochin, Kerala, India

Background: Pediatric acute and recurrent acute pancreatitis are increasingly reported from South India. Aim: To evaluate the presentation and course in a cohort of pediatric pancreatitis. Methods: 73 children (age 2-18 years) with features of pancreatitis (clinical, laboratory and imaging parameters), were followed up between January 2003 and September 2010. There were 40 males (54.79\%) and 33 (45.20\%) females. Results: 36 (49.31\%) had acute pancreatitis (AP) and 37 (50.69\%) had chronic pancreatitis (CP). Etiology included drug ingestion (3), pancreas divisum (3) and genetic mutation (1) (14 yr old boy with CP had mutations of CFTR, SPINK1, and CASR genes). The rest were idiopathic. Abdominal pain was predominant symptom in all 73 cases. Onset of pain in majority $(68.4 \%)$ was at $>10$ yrs age. Six $(8.21 \%)$ of the 73 cases had diabetes mellitus; 2 in AP and 4 in CP. 30 (81.08\%) of 37 cases with $\mathrm{CP}$ had evidence of calcification. During follow up of 36 AP, 28 patients proceeded to RAP. Further, 10 patients of the CP group had progressed from RAP after 6 months- 2 years. Average number of hospitalizations was $1.63 \pm 1.18(1-4)$ for acute, $2.81 \pm 1.81(1-7)$ for RAP and $2.35 \pm 3.44(1-18)$ for CP. Average number of attacks in RAP was $3.54 \pm 1.52$ (range $2-18$ ) yrs. Complications were seen in $33.33 \%$ of AP and $16.2 \%$ of CP. $33.33 \%$ of the AP patients gave family history of $\mathrm{CP}$ or diabetes mellitus. The single patient with mutations in 3 genes gave history of his father dying of pancreatic cancer complicating CP. Conclusion: Pancreatitis, particularly acute and recurrent, is an increasing problem in pediatric age group in South India. This follow up shows that rapid progression from AP to RAP and further to $\mathrm{CP}$ was common in this cohort and might partly explain the large incidence of $\mathrm{CP}$ among young persons in this population. 


\section{AP-C17 \\ Efficacy of Percutaneous Catheter Drainage and the Factors Leading to Surgery in Severe Acute Pancreatitis}

\author{
Y.R. Babu, R. Gupta, M. Kang, D.K. Bhasin, S.S. Rana, \\ R. Singh
}

Divisons of Medical and Surgical Gastroenterology, and Radiology, Postgraduate Institute of Medical Education and Research, Chandigarh 160012, India

Background: The standard management in patients with severe acute pancreatitis (SAP) was initial conservative management followed by operative intervention. Aim: To assess the safety and efficacy of percutaneous catheter drainage (PCD) in SAP and to determine the various factors leading to surgery. Methods: Ongoing prospective study from April 2008 to December 2009. All consecutive patients (70) with SAP were recruited. As a step up approach, patients were initially managed conservatively and as per the indication underwent PCD and surgery. The outcome and various clinical, laboratory and radiological factors in patients managed with different modalities was compared. Results: Of the 70 consecutive patients with SAP, 14 patients were managed conservatively, 29 underwent $\mathrm{PCD}$ alone and the remaining 27 underwent initial PCD followed by surgery. Achievement of sepsis control with PCD was $62.5 \%$ (27 patients in the PCD group and 8 in surgery group). The curative efficacy of PCD alone was in 27 patients $(48 \%)$. Disease specific mortality in patients requiring surgery was $11 / 27(40 \%)$ and in patients requiring $\mathrm{PCD}$ alone was $2 / 29$ (6.8\%). Mean number of PCD catheters per patient in PCD alone group was $1.59 \pm 0.73$, and in surgery group was $1.81 \pm 0.92$. On univariate analysis the factors predicting surgical intervention were initial APACHE II score (p-0.001), APACHE II score at intervention (p-0.000), Renal failure (p-0.004), Respiratory failure (p-0.004), Polymicrobial bacterial culture (p-0.003), admission CTSI score (p-0.04), Duration of TPN requirement (0.034), E.Coli in bacterial culture ( $\mathrm{p}-0.02)$. Conclusions: Percutaneous catheter drainage is a safe and effective technique as a component of Step-up approach in the management of SAP and the sepsis was controlled in $62 \%$ and complete recovery was achieved without surgical intervention in $48 \%$. On multivariate analysis, the factor predicting the surgical intervention was polymicrobial bacterial culture $(p=0.04)$.

\section{AP-C18 \\ Prospective Comparison of Long Term Outcomes in Patients of Severe Acute Pancreatitis Managed by Operative and Non- operative Means}

\author{
P. Chandrasekaran, R. Gupta, M. Kang, D.K. Bhasin, \\ S.S. Rana, S. Rana, R. Singh
}

Division of Surgical Gastroenterology and Departments of General Surgery, Gastroenterology and Radiology, Postgraduate Institute of Medical Education and

Research, Chandigarh, India

Background: The present study reports the long term functional and morphological changes following severe acute pancreatitis and compares the patients managed by operative and non-operative methods. Methods: 29 patients who had completed one year of follow up after recovery from attack of acute pancreatitis were included. 72 hours fecal fat analysis, glucose tolerance test and pancreatic morphology (MRI) were examined and recurrences if any were noted. Results: Etiology was alcohol (14), gall stones (10), and idiopathic (5). Twelve patients were managed conservatively, out of which seven patients needed pigtail drainage. 17 patients required surgery. Mean follow up was 26.9 months. 18 patients $(62 \%)$ had readmissions. 11 patients $(37.9 \%)$ had exocrine deficiency and 14 patients $(48.3 \%)$ had endocrine deficiency, 7 patients had both. $82.8 \%$ patients had morphological changes in pancreas. Patients undergoing necrosectomy had significantly higher incidence of endocrine dysfunction $(70 \%$ of patients in surgical group and $16.5 \%$ in non-operative group $(\mathrm{p}=0.008)$. Exocrine abnormality was also higher in surgical group compare to non-operative group ( $58 \%$ versus $8.4 \%, \mathrm{p}=0.007)$. Morphological changes in the pancreas were also higher in the operated group but were not statistically significant. Conclusion: Patients of severe acute pancreatitis on follow up had significant functional and morphological changes with $62 \%$ patients requiring readmission. Patients who were managed conservatively had lesser incidence of exocrine and endocrine deficiencies, though there was no significant difference in pancreatic morphological changes.

\section{AP-C19 \\ Comparative Evaluation of Transpapillary Drainage with Nasopancreatic Drain and Stent in Patients with Large Pseudocysts Located Near Tail of Pancreas}

\author{
D.K. Bhasin, S.S. Rana, M. Nanda, V.S. Chandail, R. Gupta, \\ M. Kang, B. Nagi, S.K. Sinha, K. Singh \\ Departments of Gastroenterology, Surgery and \\ Radiodiagnosis, Postgraduate Institute of Medical \\ Education and Research, Chandigarh, India
}

Background: Endoscopic transpapillary drainage is usually not advocated for large pseudocysts for fear of infection. We compared efficacy of transpapillary drainage with nasopancreatic drain (NPD) or stent alone in large pseudocysts $(>6 \mathrm{~cm}$ ) located near tail of pan- 
creas. Methods: In a prospective study, a 5 Fr stent/NPD was placed across/near pancreatic duct disruption in 11 patients ( 9 chronic, 2 acute pancreatitis) with large pseudocysts located near tail of pancreas. The patients were followed up for resolution of pseudocyst, need for surgery and complications. Results: Pseudocysts diameter ranged from $7-15 \mathrm{cms}$. An attempt to place NPD was made in 5 patients and a stent in 6 patients. In NPD group, deep cannulation could not be achieved in 1 patient, was treated successfully with percutaneous drainage. In 4 patients with partial duct disruption, NPD was successfully placed bridging disruption and all had resolution within 6 weeks. In stent group, 5 had partial and 1 had complete duct disruption, who later recovered by placement of a stent. Of 5 patients with partial disruption, one recovered uneventfully at 6 weeks with stent bridging disruption. Other 4 patients (bridging stent in 3) developed febrile illness and infection of pseudocyst. They required additional percutaneous drainage and antibiotics. There was no recurrence ofpseudocystsoverfollowupof 16.4months. Conclusion: Endoscopic transpapillary drainage with NPD bridging disruption is associated with good outcome in patients with large pseudocysts at tail end of pancreas. However, there was increased frequency of infection when stent was used for drainage.

\section{AP-C20 \\ Role of Endoscopic Ultrasound (EUS) in Evaluation of Idiopathic Acute Pancreatitis (IAP)}

\author{
S.S. Rana, D.K. Bhasin, K.Singh
}

Department of Gastroenterology, Postgraduate Institute of Medical Education and Research (PGIMER), Chandigarh, India

Background and Aims: No cause is found in $10-30 \%$ of patients with acute pancreatitis and finding a cause in these patients is a daunting task. We evaluated the efficacy of EUS in patients with IAP. Methods: Retrospective analysis of patients referred for EUS for evaluation of IAP with normal CT and MRCP was done. Chronic pancreatitis was diagnosed when $>5$ EUS criteria were present. EUS was performed after 3 months of an episode of acute pancreatitis. The diagnosis obtained on EUS was confirmed by surgery or ERCP or clinical follow up. Results: Over a twenty two month period, 30 patients of IAP with no underlying cause found on transabdominal ultrasound or CT or MRCP were evaluated. $15 / 30(50 \%)$ of the patients had biliary tract disease on EUS (cholelithiasis in 2, gall bladder sludge in 8 , choledocholithiasis in 1 and common bile duct sludge in 4 patients). One patient had $8 \mathrm{~mm}$ tumor in the head of pancreas. No underlying cause could be found on EUS in 14 (46.6\%) patients. Of these 14 patients, 7 patients had EUS features of chronic pancreatitis and on follow up had recurrent episodes of abdominal pain without elevation of pancreatic enzymes. The remaining 7 patients with normal pancreas subsequently remained well (followup: 5-22 months). Conclusion: EUS is a useful investigational modality for evaluation of patients of IAP.

\section{AP-C21 \\ Step Up Approach in Emphysematous Pancreatitis}

R. Gupta, Y.B. Raghavendra, M. Kang, D.K. Bhasin, S.S. Rana, R. Singh

Division of Surgical Gastroenterology and Departments of General Surgery, Radiodiagnosis and Imaging, and Gastroenterology, Postgraduate Institute of Medical Education and Research, Chandigarh, India

Background: Emphysematous pancreatitis (EP) is considered a virulent form of infected pancreatic necrosis (IPN). The objective of this study was to evaluate the role of step up approach in the management of EP. Methods: 70 consecutive patients with severe acute pancreatitis were recruited in this prospective study between April 2008 and December 2009. Patients of IPN were analysed for presence ofgas in the pancreatic or peripancreatic area. EndPoints: Effectiveness of step up approach in EP and compare result with non emphysematous IPN. Results: There were 53 patients of IPN enrolled during this study period. 16 of these patients $(30.1 \%)$ had EP. All patients in EP group required percutaneous catheter drainage (PCD), $8(50 \%)$ were managed with PCD alone, while the remaining $8(50 \%)$ required necrosectomy after initial PCD. In non emphysematous IPN group $(\mathrm{N}=37)$, one patient $(2.7 \%)$ was managed conservatively, $17(45.9 \%)$ managed with PCD alone, and 19 (51.3\%) underwent necrosectomy after initial PCD. No statistically significant difference was present in the two groups as regards etiology $(p=0.15)$, percentage of necrosis $(p=0.74)$, APACHE II score $(p=0.19)$, CTSI score $(p=0.78)$, number of organisms grown $(p=0.26)$, presence of fungemia $(p=0.12)$, multiorgan failure $(p=0.36)$, outcome $(p=0.58)$, therapeutic intervention $(p=0.3)$. Conclusion: Step up approach could be employed in EP with similar results as in non-emphysematous IPN.

\section{AP-C22 \\ Role of Perfusion CT in Evaluation of Early Acute Pancreatitis and Correlation with Clinical Outcome}

A. Sagar, M. Kang, D. K. Bhasin, R. Gupta, A. Bhalla, S.S. Rana, N. Khande/wal

Departments of Radiodiagnosis and Imaging, Gastroenterology, Division of Surgical Gastroenterology, Department of General Surgery and Internal Medicine, Post Graduate Institute of Medical Education and

Research, Chandigarh, India

Background: Perfusion imaging by multidetector computed tomography (MDCT) is a new and upcoming modality to measure tissue perfusion of the pancreas in acute pancreatitis. Aims: To evaluate efficacy of CT perfusion for detection of early ischemic changes in patients with acute pancreatitis and to assess the correlation of CT perfusion measurements with the clinical outcome. Methods: 39 consecutive patients with clinical diagnosis of acute pancreatitis presenting within 72 hours of symptom onset were enrolled in the study. A control group of 15 patients was also enrolled. Eleven perfusion 
parameters were evaluated for both the groups: EFP (extraction fraction product), FP (blood flow), VP (blood volume), PS (permeability surface product), KEP (outflow rate), VE (leakage rate into extravascular space), E (extraction measure), MTT (mean transit time), DT (delay time), PEI (peak enhancement intensity) and TTP (time to peak). The severity of disease was assessed using CTSI (Balthazar) and modified CTSI (Mortele) score. The following parameters of clinical outcome were recorded: duration of hospital stay, need for intervention, development of infection, organ failure and mortality. Results: EFP, FP, VP, KEP and PEI were significantly lower in the study group as compared to the control group. VE, TTP and E were higher in the study group. EFP, FP, VP, KEP and PEI correlated well with regard to need for percutaneous intervention. FP, VP, KEP and PEI correlated with duration of hospital stay. EFP correlated well with occurrence of infection. Between mild and severe pancreatitis groups the parameters EFP, FP, VP, KEP, VE, E, PEI and TTP were statistically significant. Conclusion: Our data suggests that pancreatic perfusion measurement using MDCT could help in early assessment of severity and predict clinical outcome of acute pancreatitis.

\section{AP-C23 \\ Severe Pancreatitis: Late Operation with Bad Result}

\section{S. Galeev, J. Knyazeva, F. Bhat, Y. Abdullaev}

General Surgery Department, Saint-Petersburg State Medical Academy, Russia

Introduction: According to current IAP recommendations patient with necrotizing pancreatitis should be operated on the $3 \mathrm{~d}$ or 4th week. Contrary, septic complications may manifest much earlier in the course of the disease. Methods: Records of 54 severely ill patients with necrotizing pancreatitis were studied. 17 of patients $(31,5 \%)$ presented 2 or more negative prognostic signs at admission (extended pancreatic necrosis, infection, pancreatitis-specific systemic disorders), indicating fulminant pancreatitis. 47 were operated early: within 2 weeks after onset of symptoms - group 1, other (7) underwent delayed surgery - group 2. Groups were compared in respect of systemic disorders progression and disease outcome. Results: Severity of systemic disorders (according to Marshall score) at admission didn't differ between groups. Despite on conservative therapy, gr. 2 patients possessed more pronounced disease severity at the time of surgery, indicating organ failure progression $(5,3 \pm 2,5$ vs $3,2 \pm 2,5 ; p<0,05)$. Delayed operation allowed bloodless, adequate debridement, but didn't prevent systemic disorders postoperatively. Mortality rate in gr. 2 reached $71,4 \%$, so it was unacceptably high. Deaths in all patients of abovementioned series were closely confined to sepsis-induced organs failure. Although mortality rate differences in groups didn't reach statistical significance $(29,8 \%$ vs $71,7 \% ; p<0,1)$, common sense of surgeon dictates: too late operations (3-4 week) as bad as too early (first days from disease onset). Conclusions: Evolution of inflammatory-destructive process in the abdomen can't be strong point for making decision about timing of operation in most severe cases. Time of surgery probably should be reevaluated in patients developing sepsis early in the course of pancreatitis.

\section{AP-C24 \\ Role of Endoscopic Ultrasound in Identification of Etiology and Structural Changes During Acute Pancreatitis}

\section{Sharma, P. Mohan}

Department of Gastroenterology, Jaswant Rai Specialty Hospital, Meerut, Uttar Pradesh, India

Background and Aim: The aetiology of acute pancreatitis is not evident in 10-30\%. The aim of our study was to determine the role of Endoscopic ultrasound (EUS) in finding aetiology and structural changes during the first attack of acute pancreatitis. Methods: Retrospective analysis of patients with acute pancreatitis was done between September 2005 and August 2010. Baseline demographic characteristics and biochemistry including complete hemogram, blood sugar, urea, serum creatinine, liver function tests, amylase, lipase, calcium, and triglycerides were recorded. All patients underwent transabdominal ultrasonogram and or contrast enhanced $\mathrm{CT}$ of abdomen. Patients with first attack of pancreatitis, where aetiology was not known and in whom EUS was done during the acute episode were included for analysis. Those with previous attacks of pancreatitis or with an established aetiology were excluded. Results: Out of 152 patients admitted with acute pancreatitis, 44 fulfilled the inclusion criteria. Endoscopic ultrasound was able to establish the etiology in 27 patients. (Biliary sludge - 12; CBD stone - 8; Stone impacted at ampulla - 3; Migrated stone in pancreatic duct - 2; Pancreatic sludge - 1; and hypertrophy of prepapillary sphincter -1). The major structural changes on EUS were diffuse enlargement of the gland with variable echoes inside (hypo, hyper or mixed). Thickening of the lesser omentum and tracking of fluid within the pancreatic parenchyma were seen in 3 patients with mild pancreatitis and a normal CT. Conclusion: EUS was successful in establishing aetiology in $61.4 \%$ of acute pancreatitis. The structural changes on EUS in an otherwise normal pancreas by CT were thickening of lesser omentum and fluid tracking within the pancreas.

\section{AP-C25 \\ Evaluation of Complications of Pancreatitis: Role of Multi Detector CT Scan}

R. Lakhia, L. Patel

Department Radiology, VS Hospital, NHL Medical College, Ahmedabad, Gujarat, India

Background: Pancreatitis is frequently encountered in routine clinical practice. Radiology imaging has a vital part in management because it facilitates identifying the course of diseases including development, progress and complications. Aims: The purpose of our study is to offer a synopsis of imaging findings in patients presented with complications after pancreatitis, reporting the experience with multi detector CT scan (MDCT) in complications of pancreatitis. Methods: All patients referred to our center for MDCT evaluation of pancreatitis between June 2009 and September 2010 were considered in the study. Patient's history, ultrasonography findings, endoscopy findings, and follow up were received. Results: A total 
of 50 patients: $72 \%(36 / 50)$ male, mean age 57 years (range, 26-78) made up our studied population. MDCT demonstrated normal findings in $18 \%(9 / 50)$, pancreatic pseudo cyst in $28 \%(14 / 50)$, pancreatic abscess in $4 \%(2 / 50)$, necrotizing pancreatitis in $22 \%(11 / 50)$ and acute on chronic pancreatitis in $10 \%(5 / 50)$, pancreatic phlegmon in $2 \%(1 / 50)$, peri pancreatic fluid with local lymph nodes in $6 \%(3 / 50)$, pseudo aneurysm in $2 \%(1 / 50)$, vein thrombosis in $2 \%$ $(1 / 50)$, and pleural effusion in $6 \%(3 / 50)$. Sensitivity of MDCT for diagnosis of complication of pancreatitis was $70 \%$, the specificity being $95 \%$ and accuracy being $90 \%$ for MDCT. Conclusion: MDCT is protected and precise diagnostic test and can play an essential role in timely diagnosis of patients with complications of pancreatitis. The high-quality performances of MDCT set aside early diagnosis of those symptomatic patients since inflammation of pseudocyst formation, peri-pancreatic fat, vein thrombosis, micro-aneurysm and fluid collections seems to be almost specifically suggestive of complications of pancreatitis.

\section{AP-C26 \\ SIRS and Persistent Early Organ Failure: Redefine High-risk in Acute Pancreatitis?

\author{
M.N. Saravanan, R. Maheshwari, B. Singh, \\ K. Ravindranath, R.V. Raghavendra Rao \\ Department of Surgical Gastroenterology, Global \\ Hospitals, Hyderabad, Andhra Pradesh, India
}

Background: Mortality in acute pancreatitis (AP) is related to systemic inflammatory response syndrome (SIRS) and organ failure-its timing, severity and reversibility. Aim: To assess the significance of early SIRS and the timing of organ failure in predicting outcomes of AP. Methods: Data from consecutive patients with AP treated at a tertiary referral institute between January 2009 and September 2010 was collected. Early organ failure (EOF) was defined as the development within 5 days from the onset of symptoms of 1 or more of the 1992 Atlanta parameters. Transient EOF was defined as OF of less than 48 hours duration. Outcomes were measured in terms of necrosis and mortality. Predictive accuracy was measured by the area under the receiver-operating curve (AUC). Results: Of the 63 patients with AP (mean age 41.5, 88.9\% males), 22.2\% developed OF, 36.5\% developed necrosis, $34.9 \%$ had early SIRS and $3(4.8 \%)$ died. SIRS was seen in all the 3 deaths that occurred $(p=0.039)$. AUC's for SIRS, Atlanta and CTSI in predicting mortality were 0.84 , 0.82 and 0.85 respectively, suggestive of good correlation. Six patients had persistent EOF, of which 2 died. The only other death, which occurred, was in a patient with persistent late OF. In persistent OF, early $(p=0.54)$ or late $(p=0.4)$ did not seem to alter the outcome. Transient or persistent OF did not achieve statistical significance in determining mortality $(p=1.0)$ or necrosis $(p=0.50)$. However, OF alone faired better than Atlanta and computed tomography severity index (CTSI) in predicting mortality (AUC - 0.91, 0.83 and 0.85 , respectively). Conclusion: SIRS is an early indicator of severity in AP. The failure of timing and reversibility of OF in refining outcomes needs confirmation in a larger cohort.

\section{AP-C27 \\ Case-matched Comparison Study of the Necrosectomy by Retroperitoneal Approach with Transperitoneal Approach for Necrotizing Pancreatitis in Patients with CT Severity Score of 7 and Above}

\section{P. Senthil Kumar, V. Vimalraj, M. Madavan, E. Selvakumar,} R. Sukumar, S. Sukumar, G. Rajarathinam, L. Anand, D. Jyothibasu, R. Ravi, S. Jeswanth, P. Ravichandran, R. Surendran

Institute of Surgical Gastroenterology and Liver Transplantation, Government Stanley Medical College, Chennai, India

Background: Minimally invasive necrosectomy through a retroperitoneal approach has shown promising results for the treatment of necrotizing pancreatitis. There is however, little evidence from comparative studies in favor of these techniques over laparotomy. Aim: To perform a case-matched comparision of patients with necrotizing pancreatitis who underwent necrosectomy by the retroperitoneal approach with transperitoneal approach, thus minimizing the risk of confounding and selection bias. Methods: Between 2006 and 2010,85 patients were admitted with pancreatic necrosis. Each of the 15 patients who underwent necrosectomy by reteroperitoneal approach using a small flank incision (RP group) was compared with 15 of those treated with necrosectomy by transperitoneal approach (TP group). These patients were matched for the age (+10 years), status of infection, CT severity score $(+2$ points), pre operative organ failure and timing for surgery ( +7 days). Results: The pre operative characteristics such as age, sex, pre operative intensive care unit (ICU) admission, ICU stay, APACHE-II scores, CT severity index, organ failure, timing of surgery and the presence of infective necrosis were matched. Postoperative complications occurred in 4 patients $(26.6 \%)$ in the RP group and 8 (53.3\%) in the TP group. Reintervention, either surgical or radiological for post operative complications or further necrosectomy was required in 4 patients $(26.6 \%)$ in the RP group and $7(46.6 \%)$ in the TP group. Post operative new-onset organ failure occurred in 3 patients $(20 \%)$ in the RP group and $3(20 \%)$ in the TP group. One patient $(6.7 \%)$ died in each group. Conclusions: The retroperitoneal approach for pancreatic necrosectomy through a small flank incision was associated with a lower morbidity rate compared to transperitoneal approach. However this still requires to be confirmed by further randomized studies. 


\section{AP-C28 \\ Evaluating Endotherapeutic Modalities in Pancreatic Ascites and Pleural Effusion}

\author{
P. Murali Krishna, A. Aditya, A. Sandeep, Md. Akbar, \\ N. Srinivas, L. Girinadh \\ Department of Gastroenterology, Andhra Medical College, \\ Vishakapatnam, India
}

Background: Massive pancreatic ascites and pancreatic pleural effusion are uncommon but well recognised complications of Acute Pancreatitis (AP) and Chronic Pancreatitis (CP) and is treated conservatively, by endotherapy or by surgery. Due to low incidence and few studies conducted there is paucity of data comparing the efficacy of various management modalities. Aim: To study clinical profile and evaluate the efficacy of therapeutic modalities of pancreatic ascites. Methods: 22 patients with pancreatic ascites were treated conservatively for 3 weeks and in the absence of resolution, were subjected to endotherapy by sphincterotomy and/or stent placement. Clinical end points were resolution of ascites and need for surgery. Results: All 22 were male with age range of 15 to 58 years. Etiology in 21 cases was alcohol use, in 1 it was unknown (CP-15, AP-7, with pseudocyst-5, with effusion-4). Of the 22 cases, 6 improved with conservative management, one died. 15 cases were subjected to ERCP with success in 11. Duct disruption was identified in 14 cases (body-6, tail7, genu-1). Endotherapy caused resolution in 2 of 4 cases with AP and 9 of 11 cases with CP. Sphincterotomy was done in all, stent was placed in 7 cases, all of which bridged duct disruption. All the patients with stent improved. Of the 8 patients with only sphincterotomy, 4 improved. Of the 5 cases with pseudocyst, 3 improved with sphincterotomy and stent, 1 required surgery and the other died. Of the 4 cases with associated pleural effusion, 1 improved with conservative management, 1 with sphincterotomy, 2 with sphincterotomy and stent. Conclusion: Our experience shows that pancreatic endotherapy is an excellent option for management of pancreatic ascites. Sphincterotomy with stent was more successful than sphincterotomy alone.

\section{AP-C29 \\ Conservative (Non-Surgical) Management of Emphysematous Pancreatitis (EP)}

N. Nadkarni, S. D'Cruz, R. Kaur, A. Sachdev

Department of Medicine, Division of Gastroenterology, Gian Sagar Medical College and Hospital, Chandigarh, India

Objectives: To assess the outcome of patients with EP on conservative management. Methods: EP was diagnosed on the basis of air in and around the pancreas without any antecedent percutaneous or radiological intervention by means of CECT abdomen. Ranson's and APACHE II scores were determined. These patients were managed with standard protocol. Ultrasound guided percutaneous drainage of PFC were done in those patients in whom fever persisted despite adequate antibiotic coverage. Patients with no evidence of organ dysfunction were continued on conservative management.
Those with persistent organ dysfunction were subjected to surgical necrosectomy if required. Out come was assessed with respect to mortality rate. Results: Of the eight cases of EP diagnosed all had CTSI 10. The APACHE II score at presentation was $11 \pm 3.6$ and Ranson's score was $4.2 \pm 1.3$. None of these patients had organ dysfunction at presentation. Patients were started on prophylactic antibiotics pending $\mathrm{C} / \mathrm{S}$. Culture of the pancreatic aspirates revealed E.coli in $3 / 8$, P. aeuruginosa in $3 / 8$ and K.pneumoniae in $2 / 8$ patients (all monomicrobial infection). Antibiotics were changed as per culture sensitivity report (piperacillin, imipenem, ciprofloxacin). Two patients had persistent fever and in addition required percutaneous aspiration of infected collection. $6 / 8$ patients showed complete recovery by 4.5 weeks. One patient showed delayed recovery by eighth week. Another developed late onset organ dysfunction requiring surgical necrosectomy. However he succumbed to his illness. Thus the mortality was $1 / 8(12.5 \%)$. Conclusion: In EP without organ dysfunction conservative management may be successful, thus avoiding the need of surgery.

\section{AP-C30 \\ Urinary Trypsinogen Test in Diagnosing Acute Pancreatitis}

F. Kurti, J. Basho, E. Zaimi

Service of Gastrohepatology, UHC 'Mother Theresa'

Tirana, Albania

Introduction: Acute pancreatitis (AP) can be difficult to diagnose. Aim: To evaluate the value of urinary trypsinogen rapid test for screening of AP and to compare it with that of serum amylase and lipase for the detection of AP in patients with acute abdominal pain. Methods: We analysed 45 patients who presented at the emergency unit of UHC with acute abdominal pain for no more than 24 hours with abdominal complaints. On admission to the hospital, the urinary samples were immediately tested with a test strip incorporating monoclonal antibodies to two epitopes on trypsinogen-2, recorded a blue line when concentrations exceeded $50 \mu \mathrm{g} / \mathrm{L}$. Serum amylase values were determined quantitatively. We could not perform quantitative measurement of trypsinogen-2 concentrations. We could not perform serum lipase level in the emergency setting, but serum samples were taken immediately and stored, than analysed in an another laboratory. Results: Acute abdominal pain was due to AP in $18(40 \%)$ patients and turned out to be of extrapancreatic origin in $27(60 \%)$ patients. In the patients with AP the test strip was positive in $16(88.8 \%)$ and negative in $2(11.2 \%)$, whereas in the 27 extrapancreatic patients there were $3(11.1 \%)$ false positive. We also examined the urine of other 18 controls, and we had only one strip-test positive. Conclusion: Trypsinogen-2 test strip showed a clinical value similar to amylase and lipase. The rapid urinary trypsinogen-2 test is a reliable and simple method for the early diagnosis of AP. A positive test identifies patients with AP in need of further diagnostic measures. The urinary trypsinogen- 2 test can be performed in health care units where laboratory testing facilities are not available. 


\section{AP-C31 \\ Endoscopic Drainage, an Effective Treatment Modality for Pancreatic Pseudocyst: SGPGI Experience}

S. Daschakraborty, A. Sehgal, S. Mohindra, P. Rai,

R. Aggarwal, U.C. Ghoshal, V.A. Saraswat, G. Choudhuri

Department of Gastroenterology, Sanjay Gandhi

Postgraduate Institute of Medical Sciences (SGPGIMS),

Lucknow, India

Background: Endoscopic transmural or transpapillary drainage is one of the effective non-surgical treatment modality for pancreatic pseudocyst. Aim: To assess the utility and safety of endoscopic drainage of pancreatic pseudocyst. Methods: We retrospectively studied the efficacy of endoscopic drainage in 51 symptomatic pancreatic pseudocyst patients admitted from 2000-2009. Results: Median age of patients was 39 years (range 18-73 yrs). 41 patients $(80 \%)$ were male. 40 patients $(80 \%)$ had associated acute pancreatitis and 11 patients $(20 \%)$ had chronic pancreatitis. Median size of pseudocyst was $14 \mathrm{~cm}$ (range $10-22 \mathrm{~cm}$ ). Lesser sac $(85 \%)$ was the commonest location of pseudocyst. The presenting symptoms were epigastric lump (72\%), early satiety (65\%) and abdominal pain (62\%). Median duration of symptoms was 12 weeks (range 4-72 wks). Forty patients $(78 \%)$ underwent cystogastrostomy. Cystoduodenostomy and transpapillary drainage was done in $12 \%$ and $10 \%$ cases respectively. Endoscopic nasocystic drainage in addition to cystogastrostomy was done in $20(40 \%)$. Seven patients $(13 \%)$ had complications related to the procedure, 4 had bleed, 2 perforations and one patient had both bleed and perforation. Stents related complications were seen in 13 cases $(25 \%)$, stent block in 12 cases and stent migration in 1 . Two patients (4\%) had recurrence of pseudocyst. Endoscopic drainage was a definitive treatment in $46(90 \%)$ patients. Over mean follow-up period of 9 months (3-30 months), 2 patients had recurrence. One patient was managed by percutaneous drainage followed by internalization. Another patient was managed by surgery. None of the patient had mortality. Conclusions: Endoscopic drainage provides a successful and safe approach for pancreatic pseudocyst management in appropriately selected patients.

\section{AP-C32 \\ Laparoscopic Pancreatic Necrosectomy - Early Experience}

\section{K.N. Palaniswami, K. Prakash, D. Varma, S. Aikot, M. Jacob \\ Department of GI Surgery, PVS Memorial Hospital, Cochin, India}

Background: Open surgery for pancreatic debridement is often associated with major morbidity such as fistulae and wound related complications. Laparoscopic pancreatic necrosectomy is a safe and promising alternative that minimizes surgical trauma and postoperative organ dysfunction in an already compromised patient. We share our preliminary experience of 4 patients who underwent Laparoscopic pancreatic necrosectomy. Methods: From December 2008 to July 2010 four male patients (mean age, 35 years) underwent laparoscopic pancreatic necrosectomy. An intraperitoneal retrogastric-retrocolic approach was used to access the pancreatic necrosis and drainage catheters were placed in the cavities to facilitate postoperative irrigation and lavage. Results: Four patients underwent laparoscopic evacuation of pancreatic necrosis. Mean interval between acute episode and surgical intervention was $42(20-80)$ days. The mean operating time was 155 minutes and mean blood loss was $250 \mathrm{ml}$. The location of necrosis was mainly in the lesser sac. There were no major postoperative complications related to the procedure itself. All patients tolerated nasojejunal feeds from first postoperative day and were on full oral feeds by second postoperative week. Only one patient underwent CT guided drainage of intraabdominal collection around the 3rd postoperative week. The mean length of hospital stay after surgery was 24.5 (21-41) days. All patients were discharged with drainage catheters in situ and the same were removed on outpatient basis when 24 hour output reached insignificant levels and ultrasound revealed no major intraabdominal collection. Conclusion: Our preliminary experience suggests that laparoscopic pancreatic necrosectomy is technically feasible and safe and is associated with good outcome.

\section{AP-C33 \\ Minimally Invasive Pancreatic Necrosectomy - Techniques and Outcomes from a Tertiary Centre}

\author{
P. Senthilnathan, A. Ramanujam, A.P. Manoj Kumar, \\ S. Rajapandian, P. Praveenraj, C. Palanivelu \\ Gem Hospital, Coimbatore, India
}

Background: Necrotizing pancreatitis with infected necrotic tissue is associated with a high rate of complications and death. Standard treatment is open necrosectomy. The outcome may be improved by a minimally invasive approach. Methods: In this single centre study we collected the data from a prospectively collected database of patients undergoing minimally invasive necrosectomy between 2006 and 2010. Totally 55 patients have undergone minimally invasive necrosectomy (44 through regular laparoscopy, 11 through retroperitoneal approach). The preoperative data, operative details, techniques adapted and post op recovery/omplications were studied and analysed. Results: Fifty five patients underwent minimally invasive approach; the procedure was completed in all successfully as shown by post op radiological investigations. Out of 44 patients treated laparoscopically 27 were approached through lesser sac, 12 through mesocolic route and 5 through paracolic route. The retroperitoneal approach was performed in a right lateral position, trocars are placed by open technique and debridement was performed with the help of intra operative USG if needed. Forty-eight of fiftyfive patients had at least one organ failure. Their mean age was 46 years, mean body mass index was 28.6 , and mean preop hospital stay was 18 days. The mean operating time was 65 mins and mean blood loss was $120 \mathrm{~mL}$. Six patients required reoperations for recurrent infective collections, 3 underwent open procedure and 3 were treated laparoscopically. One patient died of multiorgan failure on 14th POD. The mean length of hospital stay after surgery was 17.3 days. There were no wound complications. The various technical modifications which helped in achieving the desired outcomes were also noted 
down. Conclusions: The procedure is feasible and associated with a low morbidity and mortality. Pancreatic debridement with minimally invasive approach decreases the post op complications especially the wound complications and may provide a new option for the surgical treatment of selected patients with necrotizing pancreatitis requiring surgery.

\section{AP-C34 \\ Albumin Administration in Acute Pancreatitis Increases Lung and Pancreatic Damage Reversed by Nitric Oxide Synthase Inhibitor}

E.E. Abdo, A.M.M. Coelho, S.N. Sampietre, N.T. Molan, J.E. Monteiro-da-Cunha, R.A. Patzina, L.A.C. D'Albuquerque, M.C.C. Machado

Departments of Gastroenterology and Biochemistry, University of Sao Paulo, Brazil

Background/Aim: Colloid resuscitation in acute pancreatitis (AP) is a matter of controversy due to the possible deleterious effect on lung function. Previous study demonstrated that albumin administration increases lung damage in burns and that this effect can be reversed by inhibition of nitric oxide synthase (iNOS). We hypothesized that albumin administration in AP may be deleterious not only to the lungs but also to the pancreas and that those effects can be reversed by inhibition of iNOS. The aim of this study was to evaluate whether inhibition of iNOS reverses the effect of albumin on lung and pancreatic damage in AP. Methods: AP was induced in male Wistar rats by intraductal $5 \%$ taurocholate injection. To evaluate the effect of albumin on lung damage in AP, animals received IV saline (Group I) or human albumin (Group II) immediately after AP. To evaluate the effect of iNOS inhibition on lung damage in AP, iNOS specific inhibitor S-methylisothiourea (SMT) was given to animals immediately after AP. The animals were divided into groups: Group III: saline was given after AP and SMT, and Group IV: albumin was given after AP and SMT. After 12 hours serum amylase levels, lung myeloperoxidase (MPO) activity, pulmonary vascular permeability, and histological analysis in pulmonary and pancreatic tissue were determined. Results: Serum amylase levels, lung MPO activities, vascular permeability and inflammatory infiltration, and pancreatic edema were significantly increased after AP. Albumin administrated after AP increased lung permeability and inflammatory infiltration, pancreatic edema, and serum levels of amylase compared to saline administration $(\mathrm{p}<0.05)$. However, albumin administration with SMT reduced lung permeability and inflammatory infiltration, and pancreatic edema compared to albumin administration without SMT $(\mathrm{p}<0.05)$. There were no significant differences in lung MPO activities among groups. Conclusion: Restoration of extracellular fluid in AP with albumin increased the lung and pancreatic damage. Inhibition of iNOS before albumin administration reduced albumin induced damaging effects in AP

\section{AP-C35 \\ Acute Pancreatitis Associated with Acute Hepatitis E: A Series of 16 Patients}

\author{
M. Raj, C. Vasudev, G. Choudhuri, U.C. Ghoshal, \\ V. Saraswat, S. Mohindra \\ Department of Gastroenterology, Sanjay Gandhi \\ Postgraduate Institute of Medical Sciences (SGPGIMS), \\ Lucknow, India
}

Background: Acute pancreatitis (AP), an uncommon association with acute viral hepatitis, has been reported with hepatitis A or B and only rarely with hepatitis E. Aim: We present a series of 16 patients with AP associated with hepatitis E virus infection. Methods: Hospital records of patients admitted with AP to a tertiary institute (May 2004 to October 2010) were evaluated. Acute hepatitis $\mathrm{E}$ was diagnosed with presence of IgM antibody against the virus in serum. AP was diagnosed by high serum amylase or lipase ( $>3$ times upper limit of normal) and imaging (abdominal ultrasonography and/ or computerized tomography). Other common causes of AP were excluded by clinical evaluation and appropriate investigations. Results: Of 790 patients with AP, 16 (2.1\%; median age 25 years, range 16 to 54,15 male and 1 female) had hepatitis $E$ and no other cause for AP. 3 of these patients also had hepatitis A (2) and hepatitis B (1). Median interval between onset of hepatitis and AP was 8 days (range 0-35). 10 patients had mild and 6 severe AP. Complications included intra-abdominal collections (5), ARF (4) and acute lung injury (2). None needed surgical, endoscopic or percutaneous intervention. Median bilirubin level was $9.8 \mathrm{mg} / \mathrm{dl}$ (0.4 to 25), ALT 822 IU/L (54 to 4009), Prothrombin time 14.6 seconds (range 9.7 to 27.4). Median duration of hospital stay was 7 days (range 2-30). All patients, except one, recovered completely with conservative measures. The one patient who worsened had acute liver failure due to hepatitis E. Conclusion: AP associated with hepatitis $\mathrm{E}$ is not uncommon and most recover from the disease. Awareness about such an association is needed in tropical countries where hepatitis $\mathrm{E}$ is endemic.

\section{AP-C36 \\ Changing Trends in Pancreatic Necrosectomy}

\section{B.I. Babu, A.K. Siriwardena}

Academic HPB Unit, Manchester Royal Infirmary, Manchester, UK

Introduction: Open necrosectomy $(\mathrm{ON})$ is the gold standard treatment in the management of pancreatic necrosis (PN). Various techniques on minimally invasive necrosectomy (MIN) have been developed over the last decade in the management of PN. Aim: To identify changing trends in the management of pancreatic necrosis over the last decade. Methods: A 10 year search using search engine 'OVID SP' was used to trawl databases: Embase and Medline. The keyword used was 'necrosectomy' and included related terms. Articles were excluded if they were: experimental data, non-English medium, reviews, letters or neoplasm to produce a total of 186 studies. This data was scrutinised and non-relevant articles, duplicates and reviews 
were removed leaving a final study population of 86 manuscripts. We used Windsor's classification to group MIN in to combinations of endoscopic (E), nephroscope (N), laparoscope (L), videoscope (V), transperitoneal $(\mathrm{P})$, retroperitoneal $(\mathrm{R})$, transgastric $(\mathrm{G})$, percutaneous (PE). Results: An obvious trend towards publication of results on MIN has been noticed with no comparisons made to contemporary open necrosectomy.

\begin{tabular}{lll}
\hline Year & $6 \mathrm{MIN}(2 \mathrm{~L}+\mathrm{P}, 1 \mathrm{E}+\mathrm{P}, 1 \mathrm{~N}+\mathrm{P}, 1 \mathrm{~L}+\mathrm{R}$, & $7 \mathrm{ON}$ publications \\
$2000-2002$ & $1 \mathrm{~L}+\mathrm{G})$ & \\
Year & $9 \mathrm{MIN}(2 \mathrm{~L}+\mathrm{P}, 3 \mathrm{~N}+\mathrm{P}, 2 \mathrm{E}+\mathrm{G}$, & $8 \mathrm{ON}$ publications \\
$2003-2005$ & $1 \mathrm{E}+\mathrm{P}, 1 \mathrm{R}+\mathrm{PE})$ & \\
Year & $21 \mathrm{MIN}(12 \mathrm{E}+\mathrm{G}, 1 \mathrm{~L}+\mathrm{P}, 1 \mathrm{E}+\mathrm{P}, 2 \mathrm{~V}+\mathrm{R}$, & $13 \mathrm{ON}$ publications \\
$2006-2008$ & $1 \mathrm{~V}+\mathrm{PE}, 1 \mathrm{E}+\mathrm{P}, 1 \mathrm{~L}+\mathrm{P}, 1 \mathrm{~L}+\mathrm{G}, 1 \mathrm{R}+\mathrm{PE})$ & \\
Year & $17 \mathrm{MIN}(12 \mathrm{E}+\mathrm{G}, 3 \mathrm{~V}+\mathrm{R}$, & $5 \mathrm{ON}$ publications \\
$2009-2010$ & $1 \mathrm{~N}+\mathrm{R}, 1 \mathrm{R}+\mathrm{PE})$ & \\
\hline
\end{tabular}

Conclusion: Data pooling has its limitations and publication bias. Notwithstanding this, our study has shown a trend towards minimally invasive necrosectomy over the last decade. The optimal method of MIN is yet to be defined.

\section{AP-C37 \\ Analysis of the Delayed Approach to the Management of Infected Pancreatic Necrosis}

P.O. Agrawal, V.V. Gandhi, S. Philip, N.H. Doctor

Department of GI Surgery, Jaslok Hospital and Research

Centre, Mumbai, India

Background: With the emerging reports of minimally invasive surgery for infected pancreatic necrosis (IPN), the development of evidence-based guidelines for managing IPN requires a balance of data from laparoscopic and open necrosectomy series. Aim: To analyse outcomes of delayed single-stage necrosectomy after early conservative management of patients with IPN in severe acute pancreatitis (SAP). Methods: Between January 1998 and December 2009 , data of patients with SAP who developed IPN and were managed by pancreatic necrosectomy, was analysed. Results: Fifty-nine of 61 pancreatic necrosectomies were performed by open surgery and 2 laparoscopically. In 55 patients, single-stage necrosectomy could be performed $(90.2 \%)$. Patients underwent surgery at a median of 29 days (range 13-46) after diagnosis of AP. Sepsis and multiple organ failure accounted for the $9.8 \%$ mortality rate. Pancreatic fistulae $(50.8 \%)$ predominantly accounted for the morbidity. The median hospital stay was 23 days, and the median interval for return to regular activities was 110 days. Conclusion: This series provides further support to the concept of delayed single-stage open pancreatic necrosectomy for IPN. Advances in critical care, effective antibiotic therapy with carbapenems, the availability of interventional radiology and good supportive care have played a complementary role to surgery in improving outcomes in IPN.

\section{AP-C38 \\ A Comparative Analysis of CT and Clinical Scoring Systems in the Early Prediction of Disease Severity and Mortality in Acute Pancreatitis}

\section{T.L. Bollen, V.K. Singh, P.A. Banks, K.J. Mortele}

Departments of Abdominal Imaging, and

Gastroenterology, Brigham and Women's Hospital, Harvard Medical School, Boston, USA

Aim: To compare the accuracy of seven CT and two clinical scoring systems in predicting clinical severity and mortality early in acute pancreatitis (AP). Methods: Of 346 consecutive episodes of AP, there were $159(49 \%)$ episodes in 150 patients (84 men, 66 women; mean age, 54 years; age range, 21-91 years) who were evaluated with a contrast-enhanced CT scan ( $\mathrm{n}=131$ episodes) or an unenhanced CT scan ( $\mathrm{n}=28$ episodes) within 24 hours of presentation. Seven CT scoring systems as well as two clinical scoring systems were comparatively evaluated with regards to their ability to predict clinical severity and mortality of AP. Radiological data were reviewed in consensus by two radiologists, each blinded to patient outcome. Discriminant analysis was performed. Results: Clinically severe AP was diagnosed in 29/159 (18\%) episodes; 9 (6\%) patients died. The Balthazar grading system (any CT technique) and CTSI (contrast-enhanced CT only) demonstrated the highest accuracy among the CT scoring systems for predicting clinical severity and mortality, without reaching clinical significance. There were no statistically significant differences between the predictive accuracies of $\mathrm{CT}$ and both clinical scoring systems. The combination of CT scoring systems and APACHE II significantly better predicted clinically severe disease $(\mathrm{p}<0.01)$, but not mortality, compared to APACHE II alone. Conclusion: An early CT for severity assessment in AP is not recommended.

\section{AP-C39 \\ Prediction of Mortality in Acute Pancreatitis \\ Shaileshkumar M.E. , N.A. Tiwari, S. Sudhindran, O.V. Sudheer, P. Dhar \\ Department of GI Surgery, Amrita Institute of Medical Sciences, Cochin, Kerala, India}

Aim: Current methods for prediction of severity in acute pancreatitis have many limitations. BISAP score is a new simple system which has not been validated in India. We aimed to validate the accuracy of BISAP scoring system in predicting complications and mortality in acute pancreatitis and compare it with the existing scoring systems. BISAP score consists of B-Blood urea nitrogen $>25 \mathrm{mg} / \mathrm{dl}$, I-impaired mental status, S-SIRS, A-age $>60 \mathrm{yrs,} \mathrm{P-Pleural} \mathrm{effu-}$ sion. Each point is allotted a score of one. Methods: Case records of 79 patients (M: F 4:1) with acute pancreatitis who presented to our hospital from 2000 to 2007 was analysed. The BISAP score was calculated and its accuracy in predicting complications and mortality was compared with Ranson's score, CT severity index and C-reactive protein (CRP). Results: Thirty five patients (44\%) had BISAP score 
of $0,17(21.8 \%)$ each had score of 1 and 2 , and $9(11.5 \%)$ had a score of 3 . All patients with mild pancreatitis had BISAP score less than 3 . Five out of nine patients (44\%) with BISAP score of 3 and one out of $17(5.9 \%)$ with score of 2 died as opposed to none with score of 1 . Seven out of nine $(77 \%)$ patients with BISAP score of 3 had $>30 \%$ pancreatic necrosis, all of them eventually requiring necrosectomy. On comparing with other scoring systems, ROC curve demonstrated best accuracy for BISAP (AUC 0.92 vs 0.82 for Ransons and 0.52 for CRP) score in predicting occurrence of pancreatic necrosis and similar accuracy to Ranson's score in predicting mortality (AUC 0.93 vs 0.94 for Ranson, 0.59 for CRP and 0.84 for CT severity index). Conclusion: Our study shows that BISAP score is as good as Ranson's scoring and better than CT severity index and CRP in predicting mortality after acute pancreatitis. It appeared to the best in predicting occurrence of necrosis compared to other scores.

\section{AP-C40 \\ Management of Internal Pancreatic Fistula - Lessons Learnt}

\section{R.N. Menon, O.V. Sudheer, P. Dhar, S Sudhindran}

Department of Surgical Gastroenterology, Amrita Institute of Medical Sciences, Cochin, Kerala, India

Aim: Internal pancreatic fistulas (IPF) manifesting as pancreatic ascites or pancreatic pleural effusion are difficult to manage. Aim of the study was to evaluate our experience in the management of internal pancreatic fistulas. Methods: Records of 21 patients (M:F 16:5, median age 46 yrs, range 3 to 58 yrs.) with IPF (ascitic or pleural fluid amylase more than 3 times serum level) treated between Jan 2004 to October 2010 were reviewed. Results: Out of 21 patients (18 pancreatic ascites, 1 pancreatic pleural effusion and 2 combined ascites and pleural effusion), 15 had alcoholic pancreatitis while no cause could be detected in 6 . Conservative management that comprised nasojejunal feeding, octreotide and repeated paracentesis or thoracocentesis had a successful outcome in 10 patients. Endoscopic retrograde pancreatogram (ERP) was attempted in 8 patients and confirmed a ductal leak in 7 and stenting was performed in all (failed in 1, transpapillary in 2, across the leak in 5). Symptom resolution was complete in all these 7 patients although intraabdominal abscess developed in 2, necessitating percutaneous drainage in 1 and operative drainage in the other. Surgery was performed directly when MRCP suggested leak from large pseudocyst (cysto jejunostomy in 2) and a distal ductal leak (distal pancreatectomy-1) of which 2 had successful outcome while one died. Two patients who were extremely sick died on conservative management without undergoing any procedure. Conclusion: ERP and pancreatic duct stenting is an effective intervention for patients with internal pancreatic fistulas who do not respond to conservative measures. Surgery can be reserved for treatment failures or for post ERP complications.

\section{AP-C41 \\ Disconnected Duct Syndrome: Diagnosis and Management}

\author{
S. Mahesh, M.D. Gandhi, V. Lekha, B. Venugopal, \\ H. Ramesh \\ Department of GI Surgery, Lakeshore Hospital, Cochin, \\ Kerala, India
}

Background: There is scanty data in the Indian literature about pancreatic duct disconnection. Aim: Retrospective analysis of case records to identify the clinical characteristics and outcomes of management of disconnected duct syndrome (DDS) and evolve management approaches. Methods: 31 patients with DDS were identified over a 10 year period (1999-2009). There were two groups: a) acute pancreatitis related $(n=22)$ and b) pancreatic trauma-related $(n=9)$. There were 25 males and 6 females, and 7 children (age range 7 to 56; median 27). Diagnosis was achieved by ERCP in 12 cases, and by CT scan in 15 patients and fistulograms in 4 .

Results:

\begin{tabular}{lll}
\hline Treatment modality & Post pancreatitis & Post trauma \\
\hline Expectant treatment & 8 & 2 \\
Distal pancreatectomy & 3 & 2 \\
End-to-end anastomosis & 0 & 4 \\
ERCP stent placement & 9 & 4 \\
Endoscopic cystogastrostomy & 3 & 2 \\
Pancreaticojejunostomy & 4 & 1 \\
Fistulojejunostomy & 5 & 0 \\
\hline
\end{tabular}

Follow up ranged between 2 and 112 months. 20 out of 23 are well, 9 have new diabetes mellitus and 13 are on pancreatic exocrine replacement therapy. 2 patients treated by endoscopic cystogastrostomy have residual cystic collection posterior to the stomach but have mild symptoms and are on follow up. One patient who underwent fistulojejunostomy has recurrent pain and imaging showed a fluid collection near the tail of pancreas, but is being managed conservatively. Conclusions: DDS may be managed by endoscopic stenting; if it fails, then surgical resection/drainage of the tail, or fistulojejunostomy is an option. Endoscopic cystogastrostomy is not suitable for collections due to DDS especially in trauma.

\section{AP-C42 \\ Is Genetic Factor Responsible for Alcoholic Pancreatitis and Liver Disease? \\ V. Arulselvan, V. Srinivasan, K. Arunkumar, B. Sivasubramaniam, R. Surendran, V. Jayanthi \\ Department of Gastroenterology, Stanley Medical College, Chennai and Department of Preventive and Social Medicine, V.M.K.V. Medical College, Salem, India}

Background: Alcohol is known cause for chronic liver disease and chronic pancreatitis. The two diseases often do not co-exist. It is a known fact that it takes 5-6 years for an individual consuming $80 \mathrm{~g}$ alcohol per day to develop pancreatitis while it takes a much longer 
time i.e. 10 years to develop alcohol related liver disease when alcohol is consumed in the same dose. This study compares the similarities and dissimilarities on a case to case basis between alcohol related acute recurrent pancreatitis and alcohol related chronic liver disease with an intention to identify risk factors predisposing an individual to one disease but not to the other. Aim: To identify risk factors predisposing an individual to one or both i.e. alcohol related chronic liver disease and/or pancreatitis. Methods: Consecutive patients (201) attending the Dept of Gastroenterology both inpatient and outpatient and presenting with US/CECT proven pancreatitis (both acute and chronic with or without complication), liver disease (cirrhosis or hepatoma with or without complication) or a combination of pancreatitis and liver disease between January 2010 and July 15, 2010 were included in the study. Controls were men who consuming alcohol for more than 5 years, but had normal liver and pancreas on biochemical and radiological evaluation. Female gender, pancreatitis and liver disease due to other etiological factors or alcohol consumption less than 5 years were excluded from the study. Institutional ethics committee approval was obtained. Patients were categorized as Gp I: Alcoholic related pancreatitis, Gp II: Alcoholic liver disease, Gp-III: combination of pancreatic and alcohol related liver disease. Patient details included were age, baseline demographic characteristics, literacy status, per capita income, details of alcohol consumption, smoking and diet details (3 day recall). Pertaining to the disease, information on age of onset of disease, the duration of disease and date/month of diagnosis were recorded. All patients had liver function tests, baseline amylase and lipase estimation, ultrasound and CECT. Patients with features of chronic liver disease, with or without portal hypertension and or hepatoma were included. Likewise definite evidence of chronic pancreatitis on CECT was considered mandatory for inclusion in the study. Statistical analysis was done comparing all 3 groups. Results: There were 53 controls. There were 59 patients with pancreatitis (Gp-I), 97 with liver disease (Gp- II), 7 with both liver and pancreatic disease (Gp-III). The demographic profile in the three groups was similar. The socioeconomic status and smoking pattern were also similar. Pan chewing, diet pattern was similar in all groups. Age of first consumption of alcohol was also similar in all the 3 groups. The mean age of presentation in the three groups was $37.4 \pm 10.2,44.6 \pm 9.4$, and $45.3 \pm 6.9$ years respectively. Current alcohol consumption was more in Gp I (71.2\%) compared to Gp II $(50.5 \%)$, and Gp III $(57.1 \%)(\mathrm{p}<0.05)$. The mean duration of alcohol consumption was $13.1,16.5$ and 15.4 years respectively. The average current consumption of alcohol per week was similar in all, i.e. Gp II (765.3 g), Gp I and III (653 g each). Average consumption and duration of consumption of alcohol was not significantly different in all the 3 groups. Conclusion: Unlike the western reports, the age of presentation for both alcoholic liver disease and pancreatic disease is similar i.e. $13 \pm 2$ years. What predisposes an individual to develop liver or pancreatic disease is probably genetically determined.

\section{AP-C43 \\ ‘Double Helix' Paradigm in the Management of Necrotising Pancreatitis}

\author{
S. Philip \\ Medical Trust Hospital, Kochi, Kerala, India
}

Background: The treatment of necrotizing pancreatitis (NP) has changed significantly over the past few years in the light of new evidence including the multicentre Dutch and German studies. Aim: To define the current place of Minimally Invasive (MI) and Open Necrosectomy $(\mathrm{ON})$ techniques vis-à-vis Step Up (SU) and Step Down (SD) approaches by analysis of published literature till date. Methods: Multiple comparisons of primary and secondary data obtained by searches of MEDLINE for the time period 1996-October 2010 was done. Studies with original data and facts on outcome were included. Procedures were classified as ON, Percutaneous drainage (PD), Percutaneous Necrosectomy (PN), Endoscopic Necrosectomy (EN), Laparoscopic Necrosectomy (LN) and Retroperitoneal Nephroscopic and Laparostomal (RNL) techniques. There were 57 studies reporting on 729 patients undergoing MI and 657 patients in major series of ON. Results: In the PD group of 330 patients $31.21 \%$ (103) required another strategy for treatment. Within the EN group $87.5 \%$ (49) needed additional intervention. The number of patients cured by endoscopy alone was $75 \%$. Among the LN series of 67 patients $1.65 \%$ required reinterventions and $93.5 \%$ were cured by it alone. Median number of patients in LN series was 7. PN group had 74 patients of which $72.9 \%$ did not entail other modality of management and the average number of reoperations was 3.1 per patient. In the RNL cohort of 212 patients $32.7 \%$ were cured by it alone and reoperations per patient was 1.2 . In the major ON series results differed significantly depending on the type of $\mathrm{ON}$ the reoperation range was $5.3-51 \%$ and mortality $3-27.3 \%$. Conclusion: Multidisciplinary management of NP is being practiced more commonly. Neither SU nor SD approaches can be used as a blanket strategy. A policy adapted to the patient's specific needs, based.

\section{AP-C44 \\ It's Time to Get Away with Complex Multifactor Scoring Systems in Predicting Severity of Acute Pancreatitis}

V.C. Bada, K. Ravindranath

Department of Surgical Gastroenterology, Global

Hospitals, Hyderabad, India

Background: Assessment and prediction of severity of acute pancreatitis is quintessential in stratifying, prognosticating and effectively managing patients with acute pancreatitis. Till date, there is yet no ideal predictor of severity of pancreatitis. Indian data on this subject are lacking. Beyond several multi-factorial scoring systems, a multitude of biochemical variables have been studied in acute pancreatitis and are potentially good predictors of disease severity. Hypothesis: Independent biochemical markers are superior to multi-factor scoring systems in predicting severity of acute pancreatitis. Methods: All patients with acute pancreatitis were evaluated 
prospectively from Feb 2008 to May 2009. Patients presenting $72 \mathrm{hrs}$ after the onset of symptoms were excluded. Ranson's score was calculated at admission and at 48 hours. APACHE-II, APACHE-O and Glasgow scores were calculated daily for 72 hours and the most abnormal value was taken. CTSI score was calculated. CRP, IL-6 and Procalcitonin were measured at the time of admission. Final outcome in terms of Severity of Pancreatitis viz. Mild or Severe was the end point of the study against which all the variables were compared. The Atlanta Consensus Symposium definitions were used. Statistical analysis: Bivariate analysis was used to explore potential associations with severity of pancreatitis. The ROC curve and the area under the ROC (AUROC) were used to explore the ability of the various scores to predict severity. Standard cutoffs of scores reported in the literature were used where available. Results: Out of 53 patients, $32(60.38 \%)$ had mild and $21(39.62 \%)$ severe pancreatitis. The overall accuracy and AUROC for Ranson's was $81 \%$ and 0.78 ; Glasgow was $79 \%$ and 0.75 ; APACHE-II was $70 \%$ and 0.72 ; APACHE-O was $77 \%$ and 0.75 ; CTSI was $91 \%$ and 0.91 . The accuracy and AUROC for independent markers viz CRP was $92 \%$ and 0.98 ; IL-6 was $92 \%$ and 0.95 and Procalcitonin was $81 \%$ and 0.81 . The NPV for CRP, IL-6 and PCT were 100, 94 and $87 \%$ respectively. Conclusions: Independent biochemical markers are early, easy and superior in predicting severity as compared to complex, time consuming multi-factor scoring systems. Further larger studies are needed to validate the hypothesis that its time to get away with the multifactor scoring systems in predicting severity of acute pancreatitis.

\section{AP-C45 \\ Intra Abdominal Pressure in Acute Pancreatitis: Canary in a Coalmine?; Results After Rigorous Validation Protocol}

J. Jaipuria, V. Bhandari, A.S. Chawla, Y. Agarwal, H.G. Vyas, R. Chejara

Departments of General Surgery and Radiodiagnosis, V.M.M.C. and Safdarjung Hospital, New Delhi, India

Introduction: Abdominal compartment syndrome (ACS) is increasingly being recognized in severe acute pancreatitis (SAP) but most literature has highlighted this issue while retrospectively assessing intra abdominal pressure (IAP) in selected patients with SAP with paucity of data in mild disease. Many have used older definitions of intra abdominal hypertension (IAH) with instillation of larger saline volumes with IAP measurements having been done with less than the recommended ideal frequency by almost all investigators while not clearly addressing the issue of pain as a confounding measure making it confusing to recommend the target population in whom to measure and the timing of measurement of IAP which have precluded to convincingly answer if IAH is a predictor of severe disease. Aim: To evaluate IAP as a marker of severity in acute pancreatitis and to evaluate relationship between IAP and development of complications (in the initial 30 days) in patients with SAP. Methods: From Dec 2008Jun 2010, 56 patients with acute pancreatitis were included in this prospective cohort study. Definitions of Atlanta Symposium 1992 were used to diagnose and stratify patients according to severity. IAP was measured by vesical route at the time of admission and again after adequate control of pain as measured by faces pain scale to val- ues $\leq 4$ or Richmond agitation sedation scale $\leq 0$ where appropriate apart from further measurements; rigorously following latest guidelines by the World Society of the Abdominal Compartment Syndrome (WSACS). Patients were initially divided into study groups of mild and severe pancreatitis. Data was collected about length of hospital stay, development of persistent S.I.R.S. lasting $>48$ hours, organ failure (measured by Marshall Score), presence of septic complications, intra abdominal collections needing treatment, need for ICU admission, renal replacement therapy and mortality apart from standard demographic and clinical data. Patients were followed for 30 days and treated as per standard institution protocol. P value was set beforehand as $<0.05$. Results: $57 \%$ patients developed severe disease. IAH and ACS developed exclusively in 56\% and $25 \%$ patients with severe pancreatitis respectively. Pain control measures brought no significant change in measured IAP values in patients with IAH including no clinically significant change by use of neuromuscular blockade in those with ACS. Peak values of IAP were noted after a median 2 days from beginning of symptomatology with no significant difference from the admission values. Patients with IAH had very significantly higher mean APACHE 2 scores (in the first 24 hours of admission), higher CT Severity Index scores (in the scan conducted at 4-7 days), development of persistent SIRS and organ failure, development of multiple peripancreatic fluid collections/ ascites, higher presence of infected pancreatic necrosis/fluid collections and need for invasive interventions/surgical procedures, need for ICU admission and length of hospital stay when compared to patients with severe pancreatitis and no IAH. Patients with ACS experienced higher incidence of multiple organ failure, necrosis involving $>50 \%$ pancreatic parenchyma and mortality when compared to patients with SAP but without ACS. IAH compared favorably to common 'predictive' markers of APACHE 2 score and 'presence of pleural effusion' in identifying patients with SAP with lower sensitivity but higher specificity and positive predictive value. Patients with admission IAP $<7 \mathrm{mmHg}$ (duration of symptomatology $>24$ hours) enjoyed universally similar uncomplicated outcome irrespective of disease classification as mild or severe based on other criteria. Conclusion: Surveillance according to WSACS guidelines for IAH must be performed in all patients with any of SIRS, evidence of any organ failure, 'predicted' (APACHE 2 score $>8$ ) or actual severe pancreatitis as early as possible from the time of admission. IAH is a reliable marker

\section{AP-C46 \\ The Efficacy of Magnetic Resonance Cholangiopancreatography in Assessing the Etiology of Acute Idiopathic Pancreatitis}

\author{
G.N. Yattoo, G. Waiz, F. Shaheen, S.A. Zargar, G. Javaid, \\ A. Nisar \\ Sher-e-Kashmir Institute of Medical Sciences, Srinagar, \\ Kashmir, India
}

Background: Idiopathic pancreatitis may be diagnosed once all causes of pancreatitis are excluded by thorough history, metabolic profile and conventional imaging modality i.e. abdominal ultrasonography. In this study we have attempted to evaluate the cause of idiopathic pancreatitis with the help of MRCP. Methods: Fifty patients 
presenting with idiopathic pancreatitis were assessed prospectively using MRCP with strength $1.5 \mathrm{~T}$ for potential cause of the attack of pancreatitis. Results: Fifty patients presenting with idiopathic pancreatitis were assessed with MRCP. MRCP was able to establish the cause of pancreatitis in eleven (22\%) patients, as follows. CBD stone in one $(2 \%)$, Pancreatic divisum in one $(2 \%)$, GB stone in one $(2 \%)$, pancreatic duct stone in one $(2 \%)$, chronic pancreatitis in two $(4 \%)$, GB sludge in two (4\%) and anomalous pancreaticobiliary union in three $(6 \%)$ patients. Conclusion: MRCP, a non invasive and complication free imaging modality is able to establish the cause of acute pancreatitis in patients in whom the diagnosis of idiopathic pancreatitis has been made following standard investigations. Idiopathic pancreatitis should not be diagnosed unless MRCP has been performed.

\section{AP-C47 \\ Validation of Pancreatitis Outcome Prediction (POP) Score in Patients with Severe Acute Pancreatitis}

\author{
S.K. Akkim, B. Satyasree, Y. Srikanth Reddy, S. C. Paul, \\ D. Ajith Roni, P.K. Reddy Yeddula, L. Nihal \\ Departments of Medical Gastroenterology and \\ Hepatology, Narayana Medical College, Nellore, Andhra \\ Pradesh, India
}

Background: Severe acute pancreatitis carries high mortality and morbidity. Current prediction scores such as Ranson, CTSI cannot be applied immediately on admission. POP score which can be calculated on the day of admission predicted outcome more accurately. Objective: Validation of pancreatitis outcome prediction score (POP) in patients with severe acute pancreatitis. Design: This is an ongoing prospective study being conducted in a tertiary care teaching hospital from September 2009. Methods: Patients aged $>18$ years with the diagnosis of severe acute pancreatitis were included in the study. These patients were prospectively evaluated by using POP score to assess the mortality. POP score has 6 variables; Arterial pH, Age, Serum Urea, Mean arterial pressure, Pao2 / Fio2 ratio, and total serum calcium. Each variable had scores from 1- 10, with a maximum score of 40 . The mortality rate is $10 \%$ when POP score is 10 and increases to $90 \%$ when score is 30. Results: During the study period 101 patients (Males 69, Mean Age 32.1 yrs) were admitted with acute pancreatitis, 31 of them had severe acute pancreatitis and 7 patients died. POP score between $0-10$ there was no mortality, 11-20 there was 50\% mortality and scores $>21$ there was $100 \%$ mortality. Conclusion: POP score predicted mortality accurately in patients with severe acute pancreatitis. This is a one time score which can be calculated within 24 hours of admission with easily available variables.

\section{AP-C48 \\ Acute Hepatitis A Causing Acute Pancreatitis in a Child}

\section{G. Ray}

Department of Medicine, Eastern Railway Hospital, Sealdah, Kolkata, West Bengal, India

The incidence of acute pancreatitis complicating non fulminant viral hepatitis is low, mostly reported in adolescents and young adults but very rarely in children. We report the case of a previously healthy 6 year old girl who developed acute pancreatitis during course of acute viral hepatitis A. Though clinically mild, the pancreatitis was severe on CT scan [Balthazar grade D] but settled early with conservative treatment. Other possible causes of acute pancreatitis and hepatitis at this age were excluded by appropriate clinical history, serological and radiologic investigations to establish the causal relationship. The few such childhood cases [below 10 years age] have all been reported from countries endemic for water borne viral hepatitis, a fact which need highlighting since hepatitis A affects much larger number of children in these countries compared to higher age groups yet cause acute pancreatitis in only a few. Similar recent trend has been observed for hepatitis E also. Another intriguing observation is that the overall incidence of acute pancreatitis (due to all etiologies) below the age of 10 is much less than those above and even in this group, there is a decreasing trend in incidence and severity in younger age. This apparent protection in children might possibly be related to [a] Immaturity of the immune system in young children which protects the pancreas from cytokine mediated injury occurring in acute pancreatitis, [b] low pancreatotropism of the viruses in children, [c] occurrence in only those children who have a genetic predisposition to pancreatitis.

\section{AP-C49 \\ The Use of Thromboelastometry in the Assessment of Coagulation in Acute Pancreatitis \\ S.A. Welchman, M. Wilson, A. Wilkins, J. George, J. Copplestone, M. Midwinter \\ Department of Surgery, Derriford Hospital, Plymouth, UK; Department of Military Surgery, Centre for Defence Medicine, UK}

Background: Complications in acute pancreatitis (AP) are associated with the development of pancreatic necrosis. It has been postulated that this necrosis is related to a disease related coagulopathy. Aim: To assess coagulation in AP using thromboelastometry. Methods: 86 patients (serum amylase greater than $300 \mathrm{U} / \mathrm{L}$ ) were recruited within 6 hours of admission. Average age 62 years, male:female ratio 63:37. Venepuncture was performed at $0,6,12,24$ and 48 hours from admission. Thromboelastometry was performed on kaolin activated and heparinased samples using the TEG ${ }^{\circledR} 5000$ system (Haemoscope UK). The reaction time (R-time) is the time taken from onset of reaction to fibrin formation. Maximum amplitude (MA) is used to assess clot quality. There was no significant differ- 
ence in R-time between heparinase and non-heparinase samples. Results: $13 \%$ of patients had APACHE scores predictive of severe disease. $9 \%$ developed severe disease according to the Atlanta classification. $2.3 \%$ of all samples had R-time below normal range. $62 \%$ had evidence of prolonged coagulation according to R-time. There was a trend towards increased R-time across the time points assessed, although this did not reach significance. No significant difference in MA was observed over the time points measured or compared to normal range. No differences in TEG parameters were seen between patients with severe and uncomplicated disease. Conclusion: This is the first description of the use of thromboelastometry in the assessment of coagulation in AP. These data suggest that patients with AP have impaired clotting (not attributable to heparin). The clinical significance of this is unknown and requires further research.

\section{AP-C50 \\ Do Adipokines Mediate Susceptibility to Acute Pancreatitis and Worsen Severity?}

V. Muddana, G.I. Papachristou, A. Evans, D.C. Whitcomb

Division of Gastroenterology, Hepatology and Nutrition, Department of Medicine, University of Pittsburgh, Pittsburgh, PA, USA

Background: Obesity markedly increases susceptibility to, and severity of acute pancreatitis (AP), possibly through the action of adipokines. We tested the hypothesis that adipokines (Adiponectin, Resistin and Leptin) mediates some of the effects associated with obesity in AP. Methods: AP patients and controls were enrolled from the University of Pittsburgh Medical Center. Severe AP was defined by organ dysfunction with cardiovascular, pulmonary and/or renal dysfunction persisting for $\geq 48$ hours. Five functional resistin polymorphisms rs1862513 $\mathrm{C}>\mathrm{G}, \mathrm{rs} 35547567 \mathrm{C}>\mathrm{T}, \mathrm{rs} 35139946 \mathrm{C}>\mathrm{T}$, rs3745367 $\mathrm{G}>\mathrm{A}$, and $\mathrm{rs} 3745369 \mathrm{G}>\mathrm{C}$, four functional leptin polymorphisms rs7799039 A>G, rs2167270 G>A, rs4731427 C> T, and rs1137101 $\mathrm{A}>\mathrm{G}$ were evaluated by Taqman assay. Two functional adiponectin polymorphisms rs2241766 $\mathrm{T}>\mathrm{G}$ and $\mathrm{rs} 1501299 \mathrm{G}>\mathrm{T}$ were evaluated by DNA sequencing. Serum adipokines levels were measured among mild AP, severe AP and controls, using standard Luminex assay. Results: 188 patients with AP (148 MAP, 40 SAP) and 163 healthy controls were ascertained. 67 patients $(36 \%)$ and 34 controls $(22 \%)$ were obese (BMI $\geq 30 \mathrm{~kg} / \mathrm{m} 2)(\mathrm{p}=0.01)$. Resistin polymorphism rs $3745367 \mathrm{G}>\mathrm{A}$ seen in intron 2 was found to be significantly associated with susceptibility to AP with the A allele more common in AP patients than controls $(\mathrm{p}=0.0009, \mathrm{OR}=1.8)$. Restricting the analysis to lean patients and controls (BMI $<30 \mathrm{~kg} /$ $\mathrm{m} 2)$, polymorphisms $\mathrm{rs} 3745367 \mathrm{G}>\mathrm{A}(\mathrm{p}=0.0001, \mathrm{OR}=2.4)$ and rs $1862513 \mathrm{C}>\mathrm{G}$ seen in promoter region $(\mathrm{p}=0.005, \mathrm{OR}=1.9)$ were both found to be associated with susceptibility to AP and there was no significance in obese subjects. Furthermore, linkage was found between polymorphism rs1862513 and rs3745367 Lewontin's D' $=0.16$. Both adiponectin and leptin polymorphisms affected neither susceptibility to, nor severity of AP. Resistin serum levels were significantly elevated in SAP compared to MAP and controls $(\mathrm{p}<0.05)$. Conclusions: Among these adipokines, resistin appears to mediate some of the effects of obesity in susceptibility and severity to AP.

\section{AP-C51 \\ Management of Pancreatic Necrosis: Single Center Experience}

\author{
A. Sekwani, V. Singh, R. Varshney, P. Shaji, A. Jat, \\ S. Varshney
}

Department of Surgical Gastroenterology and Nutrition, Bhopal Memorial Hospital and Research Center, Bhopal, India

Introduction: Pancreatic necrosis is a devastating disease that leads to death in $10 \%$ to $50 \%$ of cases. $80 \%$ of deaths are due to sepsis and its complications. Methods: 384 patients with acute pancreatitis were encountered in our department from January 2011 to June 2010. 217 were mild and 167 severe. 61 patients underwent surgery, out of which 5 were operated within 28 days of the onset, and 57 operated 28 days or later. There were 52 males, with a mean age of 42.5 years (18-72). 32 patients had gallstones, 25 had chronic alcohol abuse (15 had gall stones as well), 1 had drug induced pancreatitis, and 4 were idiopathic. Indications for surgery were infected necrosis in 51, early SIRS with MODS in 4 patients, and pancreatic necrosis with low grade SIRS and no improvement after 4 weeks despite conservative treatment in 7 patients. Results: Post necrosectomy, eight patients had residual collections: six had percutaneous drainage and 2 were reexplored. Out of 62 patients, 3 developed intestinal fistula, which closed spontaneously, while one died who also had a pancreatic fistula. A total of 5 patients developed pancreatic fistula, one of which was high output and managed with pancreatic stenting and octreotide therapy. Four low output fistulas settled with octreotide therapy. Four patients had bleeding, two were managed conservatively; one required suture ligation after failure of radiologic embolization, and one was managed with embolization. Five patients died, 3 due to SIRS and MODS which were operated early within 4 weeks. Among the necrosectomy group, one died of cerebral stroke and infarction, and the other of complex intestinal and pancreatic fistula. Conclusion: Late necrosectomy followed by postoperative lavage is the method of choice for the surgical treatment of infected pancreatic necrosis in ANP, which can be done with minimum morbidity and mortality, and low re-exporation rate. The surgery should be delayed preferably for four weeks when possible. 


\section{Chronic Pancreatitis - Basic}

\section{CP-B1}

\section{Physicochemical Properties of Blood in Patients with Chronic Pancreatitis}

\section{N.B. Gubergrits, O.A. Golubova \\ Donetsk National University, Ukraine}

Background: Physicochemical properties of blood are integrated reflection of biochemical changes occurring in pathological conditions, including in chronic pancreatitis (CP). Aim: To study indices of tensiometry of blood serum in patients with CP. Methods: 120 patients with $\mathrm{CP}$ and 30 practically healthy persons were examined. Alcoholic CP is diagnosed in $56(46.7 \%)$ patients, biliary in $64(53.3 \%)$ patients. We studied dynamic surface tension (ST) of blood with computer tensiometer MRT-2 Lauda (Germany). Dynamics of ST was represented as tensiogram taking into account indices ST1 $(\mathrm{t}=0.01 \mathrm{~s}), \mathrm{ST} 2(\mathrm{t}=1 \mathrm{~s})$ and ST3 $(\mathrm{t}=100 \mathrm{~s})$; the angle of curved line inclination of tensiogram (ACLI) was considered. Results: Indices of ST1 and ACLI increased significantly in patients with CP, indices of ST3 decreased essentially. Only meanings of ST2 had no significant differences from the control. Numerous correlation connections between indices of tensiometry and exocrine, endocrine secretion of pancreas, with intensity of sonographic pancreas changes were revealed. The orientation of changes of physicochemical properties of blood in alcoholic and biliary CP was similar, but intensity of some indices changes was more considerable in alcoholic CP. Conclusions: Results of interphase tensiometry along with traditional tests can be used for monitoring of $\mathrm{CP}$ course and control of treatment efficacy.

\section{CP-B2 \\ Kinins of Blood in Patients with Chronic Pancreatitis}

\section{N.B. Gubergrits}

Donetsk National University, Ukraine

Background: Activation of kallikrein kinin blood system participates in pathogenesis of variety of inflammatory diseases. Value of kinin changes in chronic pancreatitis (CP) is not studied. Aim: To study condition of kinin blood system in CP. Methods: 342 patients with CP were examined. Level of prekallikrein of plasma (PP), total antitryptic activity of blood serum (TAABS), tripsin binding activity of $\alpha 2$-macroglobulin (TBA $\alpha 2-\mathrm{MG}$ ) were analyzed, ratio TAABS/ TBA $\alpha 2-\mathrm{MG}$ was determined. Cluster analysis was conducted. Results: Three types of kininograms are defined. Mobilisation of kinin system that was manifested by reduction of indices of PP, TBA $\alpha 2-\mathrm{MG}$ and increase of TAABS was revealed in $78 \%$ patients. The second type of kininogram (12\% patients) represented decrease of TBA $\alpha 2-M G$, moderate increase of TAABS and PP. The third type
( $10 \%$ patients) indicated stable condition of kinin system. It was characterized by normal level of blood PP, marginal increase of TAABS, moderate decrease of TBA $\alpha 2-\mathrm{MG}$. It is important, that every type of kininograms corresponded with certain peculiarities of clinical course of CP. Conclusions: Various types of kininograms in CP patients should be taken into account in choice of treatment tactics.

\section{CP-B3 \\ Comprehensive Screening of Chymotrypsin C (CTRC) in Tropical Calcific Pancreatitis Identifies Novel Variants}

\section{S. Paliwal, S. Bhaskar, D.N. Reddy, G.V. Rao, S.P. Singh, V. Thomas, G.R. Chandak}

Centre for Cellular and Molecular Biology, Hyderabad, and Asian Institute of Gastroenterology, Hyderabad, India

Background: Chymotrypsin C (CTRC) variants that diminish its activity or secretion were found to predict susceptibility to chronic pancreatitis (CP). Aim: To investigate variants in whole CTRC gene in a large, ethnically matched case-control cohort of tropical calcific pancreatitis (TCP) from India. Methods: We sequenced all eight exons and flanking regions in $584 \mathrm{CP}$ patients (497 TCP, 87 ICP) and 598 normal subjects and calculated statistical significance using $\times 2$ test. We predicted functional effect of novel variants using suitable online tools. We also investigated interaction of CTRC variants with SPINK1 and CTSB mutations. Results: Non-synonymous variants were detected in $71 / 584$ patients $(12.2 \%)$ and $22 / 598$ controls $(3.7 \%)$ $[\mathrm{OR}=3.62,95 \% \mathrm{CI}=2.2-6.1, \mathrm{P}=6.2 \times 10-8]$. The mutation c.703G $>\mathrm{A}$ (p.V235I) was the commonest in these patients $(28 / 575,4.9 \%)$ as compared to controls $(4 / 598,0.7 \%) \quad(\mathrm{OR}=7.6, \quad \mathrm{CI}=2.5-25.7$, $\mathrm{p}=1.01 \times 10-5)]$. Functionally established pathogenic variant, c. $217 \mathrm{G}>\mathrm{A}$ (p.A73T) was identified in $3.1 \%(18 / 584)$ patients compared to $\sim 1 \%(2 / 230)$ in controls $(\mathrm{OR}=3.63, \mathrm{CI}=0.8-22.4, \mathrm{p}=0.066)$. Several novel mutations including c.725delG (p.G242AfsX9) and c. $338 \mathrm{G}>\mathrm{A}(\mathrm{p} . \mathrm{W} 113 \mathrm{X})$ that will prematurely truncate the protein were identified. No interaction between CTRC variants and p.N34S SPINK1 or p.L26V CTSB mutations was observed. Conclusion: Our study on a large cohort of TCP patients provides evidence of allelic heterogeneity and confirms that CTRC variants play a significant role in its pathogenesis independent of the SPINK1 and CTSB. 


\section{CP-B4 \\ Genetic Alterations of Pancreatic Cancer and Chronic Pancreatitis Patients in North Indian Population}

\author{
P. Kar, S.K. Polipalli, S.A. Husain, R. Gondal, A. Agarwal \\ Department of Medicine, Maulana Azad Medical College; \\ Department of Biosciences, Jamia Milia Islamia; \\ Departments of Pathology and GI Surgery, G.B. Pant \\ Hospital, New Delhi, India
}

Background/Aim: To understand the genetic alterations of K-ras and $p 16$ gene in North Indian patients with pancreatic diseases, and the possible correlation between the presence of mutation, histopathological findings and other risk factors are assessed by standard questionnaire. Methods: A total of 65 pancreatic cancer patients and 50 chronic pancreatitis controls were inducted in the study. Out of 65 pancreatic cancer, 24 patients were female $(36.92 \%)$ and 41 patients were male (63\%) having an average age of $47.42 \pm 11.32$ years (range 22-70 years). In 50 chronic pancreatitis only 7 were female $(14 \%)$ and 43 were male ( $86 \%)$ having an average age of $36.02 \pm 10.55$ years (range 20-63 years). In the control group 35 were male and 15 were female with an average age of $36.75 \pm 11.75$ years. K-ras mutations and p16 gene were examined using the two step polymerase chain reaction (PCR) with RFLP, followed single-strand conformation polymorphism (SSCP) and automated DNA sequencing. Results: K-ras mutational analysis in surgically resected tissue samples of the pancreatic carcinoma was $72.30 \%(47 / 65)$ which was significantly higher than the chronic pancreatitis $6 \%(3 / 50)(\mathrm{P}<0.01)$ while p16 mutational analysis in pancreatic carcinoma was $58.46 \%$ ( $38 / 65)$ and chronic pancreatitis showed $8 \%(4 / 50)(\mathrm{P}<0.01)$. It was also found that K-ras and p16 mutation rate was progressively increased from normal duct at the tumor free resection margin to pancreatic carcinoma. The mutation pattern of Kras codon 12 observed in pancreatic carcinoma was GGT-GGA, while p16 gene showed CGC - CGG for aminoacid Arginine and GAG - GTG for aminoacid Glutamine to Valine GGC - GGT and this was identical in chronic pancreatitis also. In this study male showed more prevalence of p16 methylation status and mutation analysis of K-ras gene compared to female. The age $\geq 40 \mathrm{yrs}$ showed statistically higher incidence of pancreatic cancer than chronic pancreatitis $\left(\mathrm{P}<0.05^{*}\right)$. Smoking was found to be one of the risk factor for pancreatic cancer compared to chronic pancreatitis $\left(\mathrm{p}<0.05^{*}\right)$. Other possible associated risk factors like alcohol consumption, diabetes, tea, coffee, Milk products and dietary habits were found to be statistically non-significant. Conclusion: This study shows evidence of p16 methylation and mutation of K-ras gene to be statistically higher in pancreatic cancer compared to chronic pancreatitis and this suggests that inactivation of cell cycle regulator and tumor suppressor p16 and k-ras gene represent a critical early step in the genesis of pancreatic cancer.

\section{CP-B5 \\ Role of Trypsinogen Activation in the Pathogenesis of Chronic Pancreatitis}

\author{
R. Sah, R. Dawra, A. Bekolay, A. Saluja
}

Division of Basic and Translational Research, Department of Surgery, University of Minnesota, Minneapolis, MN, USA

Background: Intra-acinar trypsinogen activation is believed to be the key event in the pathogenesis of acute pancreatitis. Association of mutations related to pathologic trypsinogen activation with hereditary pancreatitis suggests that trypsinogen activation may be important in the pathogenesis of chronic pancreatitis (CP). We aimed to evaluate the role of trypsinogen activation in CP. Methods: CP was induced by caerulein $(50 \mu \mathrm{g} / \mathrm{kg}$ IP every hour X 6) twice a week for 10 weeks. We compared CP between wild type (WT) and Cathepsin B knockout (CB-/-) mice, which lack significant pathologic trypsinogen activation. Two independent experiments each with 7-8 mice/group were carried out. Results: The pancreas weight measured 39\% and $36 \%$ of that of controls in WT and CB-/- respectively. On histology, comparable acinar atrophy was seen in both groups with $500 \%$ increase in fibrosis measured by Sirius Red stained area. Cytokeratin 19 positive tubular structures increased from $3 /$ field to $28 \pm 2$ in WT and $31 \pm 2$ in CB-/- groups. Significant but similar increase in stellate cell population and activation was seen in both groups. Ki-67 positive cells (indicating cellular proliferation) increased from 3/field to $108 \pm 5$ in WT vs $98 \pm 6$ in CB-/-. Similar degree of T cell infiltrate (CD3 staining) and COX-2 upregulation was noted in both groups. Remarkably, intra-acinar NF-kB activation was seen in $\mathrm{CP}$, which was similar in both groups. Conclusion: Chronic pancreatitis in the presence (WT) and absence of significant trypsinogen activation (CB-/-) is similar suggesting that trypsinogen activation is not required for the pathogenesis of chronic pancreatitis. Furthermore, intra-acinar NF-kB activation seen in chronic pancreatitis may be responsible for its pathogenesis. 


\section{CP-B6 \\ Characterization of Pain in Caerulein Based Mouse Model of Chronic Pancreatitis}

R. Sah, R. Dawra, A. Bekolay, A. Saluja

Division of Basic and Translational Research, Department of Surgery, University of Minnesota, Minneapolis, MN, USA

Background: Chronic pain is a major problem in chronic pancreatitis (CP). The pathogenesis of pain in $\mathrm{CP}$ is not understood; therefore, animal models are crucial to study $\mathrm{CP}$ pain. Currently pain in CP has only been characterized in a rat model of CP. This model suggests that trypsin plays a possible role in CP. Here we aimed to characterize the pain response in the caerulein based mouse model of $\mathrm{CP}$ and to study the same in Cathepsin B knockout (CB-/-) mice, which lack significant pathologic trypsinogen activation. Methods: CP was induced by caerulein $(50 \mu \mathrm{g} / \mathrm{kg}$ IP every hour X 6) twice a week for 10 weeks. Pain was characterized by behavioral responses (Mouse Grimace Score (MGS) and other behavioral parameters), spinal sensory neuron activation (c-fos immune-positivity) at T9 level and by Nerve Growth Factor (NGF) upregulation in the pancreas. Results: The MGS score (0-2) in CP, calculated as increase from controls, was $0.44 \pm 0.1$. Among the behavioral parameters, hunched appearance, back-arched gait and reflexive withdrawal to deep abdominal palpation were seen in significantly higher proportion of CP animals Vs controls ( $73 \%$ vs $8 \%, 74 \%$ vs $8 \%, 64 \%$ vs $4 \%$ respectively). The number of c-fos positive neurons in the sensory area of posterior horn at T9 spinal level was increased from $9 \pm 1$ in controls to $30 \pm 3$ in CP $(p<0.05)$. NGF was overexpressed in CP animals, demonstrated by immunohistochemistry. CB-/- mice were similar to the wild type $\mathrm{CP}$ group in all of the above parameters. Conclusion: Pain can be demonstrated in the caerulein based mouse model of chronic pancreatitis, and this model can be a useful tool to study chronic pancreatitis pain. CB-/- mice have comparable pain suggesting that pain generation may be independent of trypsinogen activation.

\section{CP-B7 \\ Possible Role of ICOS Positive and IL-10 Producing Regulatory T Cells in Patients with Autoimmune Pancreatitis}

K. Uchida, T. Kusuda, H. Miyoshi, M. Koyabu, T. Ikeura, Y. Sakaguchi, K. Yoshida, T. Fukui, M. Shimatani,

M. Matsushita, M. Takaoka, A. Nishio, K. Okazaki

Department of Gastroenterology and Hepatology, Kansai Medical University, Japan

Background: Autoimmune pancreatitis (AIP) has several immunologic abnormalities, including increased levels of serum IgG4 and infiltration of IgG4+ plasmacytes. However, the role of IgG4 is unclear. Recently ICOS is paid attention to the key molecule of IL-10 secretion from Tregs. We analyzed the Tregs to clarify the role of IgG4 production in AIP. Methods: We obtained pancreatic tissue from 6 AIP and 6 cases of chronic pancreatitis. Hepatic tissue was sectioned from 16 IgG4-SC patients and 26 patients with PSC. We studied infiltrating IgG4+ cells and Tregs. ICOS positive and IL-10 producing Tregs were analyzed from peripheral blood. Results: Tregs were increased in AIP $(24.6 \pm 18.0$ cells/HPF $)$ compared with controls $(5.1 \pm 4.3$ cells/HPF). The numbers of $\mathrm{IgG} 4+$ plasmacytes were significantly higher in AIP $(18.6 \pm 10.3$ cells/HPF $)$ than in controls $(1.1 \pm 0.7$ cells/HPF $)$. In the liver, IgG4+ plasmacytes were significantly higher in IgG4-SC (16.6 \pm 7.7 cells/HPF) than in PSC (4.0 \pm 0.7 cells/HPF). Tregs in the patients with IgG4-SC (5.4 1.5 cells/HPF) were also significantly increased compared with PSC (2.0 0.3 cells/ HPF). In the patients with IgG4-SC, the numbers of infiltrated Tregs were significantly positively correlated with $\mathrm{IgG} 4+$ plasma cells $(\mathrm{R}=0.75)$. ICOS+Tregs $(3.45 \pm 1.58 \%)$ were significantly increased in comparison with hcontrol $(1.57 \pm 0.69 \%)$. Furthermore, IL10+ ICOS+Tregs $(3.81 \pm 1.52 \%)$ were significantly increased compared with healthy control $(1.38 \pm 0.64 \%)$. IL-10 producing Tregs and IgG4 also positively correlated in untreated $\operatorname{AIP}(\mathrm{R}=0.53)$. Conclusion: In AIP, Tregs may affect the switching of B cells to IgG4-producing plasmacytes. 


\section{Chronic Pancreatitis - Clinical}

\section{CP-C1 \\ Clinic Peculiarities of Chronic Pancreatitis in Patients Underwent Cholecystectomy}

\author{
N.B. Gubergrits, A. Yurjeva \\ Donetsk National Medical University, Ukraine
}

Introduction: Chronic pancreatitis (CP) frequently has been developed as a result of biliary pathology. It could be set in as prior to as after the cholecystectomy. Aim: to study clinical picture of $\mathrm{CP}$ developed after cholecystectomy. Methods: We observed 62 patients with $\mathrm{CP}$, which underwent cholecystectomy due to gallbladder disease more than a year prior to the study. Incidence of different clinical signs and palpation results were assessed. Results: Abdominal pain was the main complaint of all of the patients. 37 patients $(59.7 \%)$ had intensive pain, $20(32.3 \%)$-moderate, $5(8.0 \%)$-mild. The pain starts or increases after a meal. 17 patients $(27.4 \%)$ had pain in left hypochondrium only, 21 patients $(33.9 \%)$ - in epigastric region and left hypochondrium, 24 patients $(38.7 \%$ ) - in epigastric region and both hypochondria. Heaviness sensation and discomfort in upper abdomen were characteristic in 27 patients (43.6\%). All patients complained on dyspepsia, it was intensive in 39 patients $(62.9 \%)$, moderate-in 18 patients $(29.1 \%)$, mild - in 5 patients $(8.0 \%) .52$ patients (83.9\%) had different stool disorders. Frequently patients complained on asthenic manifestations, sleep and mood disorders. Conclusion: In CP clinical picture developed after cholecystectomy there are predominant pain syndrome with localization in left hypochondrium and epigastric region. Dyspepsia of different grade is also characteristic.

\section{CP-C2 \\ Treatment of Chronic Biliary Pancreatitis in Patients with Obesity}

N.V. Byelyayeva

Donetsk National Medical University, Ukraine

Background: Treatment of steatopancreatitis in patients with obesity is a difficult task. One of pathogeneticly grounded agents are multinutrient complexes made with using of technology «Grinization». Aim: To study efficacy of complexes «Grinization» in treatment of steatopancreatitis on the background of obesity. Methods: 44 patients with combined pathology were examined. All patients had biliary pathology, so chronic pancreatitis was biliary. All patients were diagnosed concomitant steatohepatitis. Patients were divided into groups-main and comparison. Main group included 20 patients who received traditional therapy of steatohepatitis and pancreatitis, and also Green Mix at $50 \mathrm{ml} 2$ times a day before meal and Green Pro at $25 \mathrm{~g}$ once a day during 2 weeks. Patients of comparison group received only the standard treatment. We controlled dynamics of clinical manifestations, body mass index (BMI), and biochemical indices reflecting a functional state of pancreas. Control group consisted of 30 practically healthy persons. Results: We revealed considerable advantages of treatment with using of products «Grinization» concerning reduction of dyspepsia and asthenic syndromes. Besides, significant improvement of functional state of pancreas, decrease of BMI were reached in patients of the main group. Positive dynamics of clinical manifestations also took place in patients of comparison group, however it was less evident, than in the main group. Basic therapy was less effective concerning influence on exocrine function of pancreas, on biochemical manifestations of pancreatitis also. In comparison group significant reduction of BMI was not reached. Conclusion: Complexes 'Grinization' are effective in treatment of chronic pancreatitis on the background of obesity.

\section{CP-C3 \\ The Incidence of Concomitant Pathology of Digestive Organs in Patients with Chronic Pancreatitis}

N.V. Byelyayeva

Donetsk Medical University, Ukraine

Background: Chronic pancreatitis (CP) frequently is associated with pathology of other digestive organs, which develops prior to $\mathrm{CP}$ or as a result of $\mathrm{CP}$. This fact has an influence on peculiarities of clinical picture and treatment of such patients. Aim: To study character and incidence of concomitant pathology of digestive organs in patients with alcoholic and biliary CP. Methods: We analyzed results of examination of 150 patients with alcoholic CP and 150 patients with biliary $\mathrm{CP}$. Both groups were comparatively similar by age and duration of the disease. Results: Patients with alcoholic CP had following concomitant diseases: in $82 \%$ — liver pathology (steatosis, hepatitis, cirrhosis), in $98 \%$ - chronic gastroduodenitis, in $18 \%$ - gastroduodenal reflux disease, in $12 \%$ - peptic ulcer of duodenum and stomach. In case of biliary CP it were liver pathology (steatosis, hepatitis) - 34\%, biliary pathology (cholecystitis, cholelithiasis, sustained cholecystectomy) - 100\%, chronic gastroduodenitis - 33\%, duodenal peptic ulcer - 5\%, gastroesophageal reflux disease - 23\%, chronic non-ulcerative colitis - 39\%. Conclusion: Mentioned concomitant pathology should be taken into account in diagnostics and treatment of CP.

\section{CP-C4 \\ Mineral Density of Bones in Chronic Pancreatitis}

\section{N.B. Gubergrits, I.L. Kutyrkina}

Donetsk National University, Ukraine

Background: Disorders of bones can develop in chronic pancreatitis with exocrine insufficiency of pancreas owing to malabsorption of calcium and vitamin D. These disorders can often proceed without distinct clinical manifestations. The diagnosis is defined late, 
i.e. in late stages of osteoporosis when danger of pathological fractures is high. Aim: To study mineral density of bones in chronic pancreatitis. Methods: 24 patients with chronic pancreatitis were examined. Mineral density of bones was defined with the help of densitometer Explorer QDR series Hologic (USA). Exocrine function of pancreas was estimated by results of fecal elastase test. Results: Indices of densitometry were normal in 7 (29,2\%) patients, osteopenia of I degree was revealed in $5(20,8 \%)$ patients, osteopenia of II degree - in $3(12,5 \%)$, osteopenia of III degree - in $4(16,7 \%)$, osteoporosis - in $5(20,8 \%)$. Intensity of bone changes correlated with degree of pancreatic insufficiency according to results of analysis of fecal elastase-1. In any case there were no clinical manifestations of changes of bones, though the risk of fractures was raised in $8(33.3 \%)$ patients. Conclusion: The normal mineral density of bones presented less, than in one third of patients with chronic pancreatitis. This indicated of necessity of timely carrying out of densitometry and early prescription of adequate dose of Creon in order to avoid of pathological fractures.

\section{CP-C5 \\ Digestive and Absorptive Functions of Small Intestine in Patients with Chronic Pancreatitis}

\section{N.B. Gubergrits, Y.V. Linevsky \\ Donetsk National Medical University, Ukraine}

Background: Patients with chronic pancreatitis (CP) and exocrine insufficiency of pancreas in most of cases have secondary enteritis that is accompanied by digestive dysfunction of small intestine, syndrome of bacterial overgrowth. This leads to development of malabsorption and decrease of efficacy of substitutive enzyme therapy. Aim: To study digestive and absorptive functions of small intestine in pancreatic insufficiency in patients with CP. Methods: 63 patients with $\mathrm{CP}$ were included in trial. Presence of exocrine insufficiency of pancreas was confirmed by results of fecal elastase test. Aspiration biopsy of mucous membrane of small intestine was taken. Amylolytic (by Smyth-Roe) and lipolytic (by Ugolev-Nurks) enzyme activity, the activity of lactase (by Linevsky), saccharase (by Ugolev-Iezuitova), maltase (by Linevsky), glycine-L-leucinedipeptidase (by UgolevTimofeeva), monoglyceridlipase (by Ugolev-Chernyakhovskaya) and alkaline phosphatase (by Fomina-Mihlin) were examined in homogenate of taken mucosa samples. An absorptive function of small intestine was evaluated by test with D-xylose. Results: Amylolytic and lipolytic activity of membrane and cavitary digestion was increased. Also a reduced production of monoglyceridlipase was determined. Increased production of saccharase, reduced level of glycine-L-leucine dipeptidase in mucous membrane, and growth of alkaline phosphatase contents was obtained. Insufficiency of small intestine absorptive function, syndrome of bacterial overgrowth as a result of unusual microorganisms existence in small intestine were revealed. Conclusion: Secondary disorders of digestive and absorptive functions of small intestine develop in patients with pancreatic insufficiency due to CP. The disorders observed in small intestine should be taken into account of practical doctor because these changes indicate on necessity of treatment correction.

\section{CP-C6 \\ Surgery in Chronic Pancreatitis with Combined Pancreatic and Biliary Obstruction}

\section{A. Klymenko, V. Klymenko \\ Zaporizhzhya State Medical University, Ukraine}

Introduction: Surgery in chronic pancreatitis (CP) with pancreatic and biliary obstruction is a complex and difficult aspect in pancreatic surgery. Good results of the surgery strongly depend of an adequate operative technique that should respect the principle of fast and entire correction of the disease. Objectives: To develop and research a new type of surgery in chronic pancreatitis that instantly and simultaneously corrects pancreatic and biliary obstruction. Methods: 16 patients were analysed (14(87,5\%) men, 2(12,5\%) women), age 35-62. Mean age was 48 . They all had severe forms of chronic pancreatitis (calculose, pseudotumorouse, fibrose-cystic) having led to pancreatic and biliary obstruction. Wirsung's duct diameter was 5-12 mm. Obstructive jaundice presented with the level of bilirubin 38-112 mkmole/l high. Alcohol CP was in 14(87,5) patients, biliary $\mathrm{CP}$ - in $1(6,25 \%)$, idiopathic - in $1(6,25 \%)$. Patients were examined with US, CT, ERCP, intraoperative US, videogastroduodenscopy, IgG, rheumatoid factor, operative biopsy. All the patients have undergone new technique - longitudinal total pancreatowirsungoduodenopapillotomy with simultaneous cut of the posterior common bile duct wall in its intrapancreatic part at posterior medial side and formation of Roux-en-Y pancreatojejunoduodenostomy. Results: Mortality rate was $0 \%$. Postoperative complications were in $3(18,7 \%)$ patients (pneumonia, wound infection). Abdominal pain and jaundice disappeared in all the patients. In 4-6 month postoperatively pancreatic head size was close to normal $(30,1+0,9 \mathrm{~mm})$ in $14(87,5 \%)$ patients. In $12-16$ month diabetic patients remained $2(12,5 \%)$ of $11(68,8 \%)$. Only $3(18.8 \%)$ patients still used enzymes. Conclusion: Longitudinal total pancreatowirsungoduodenopapillotomy with simultaneous cut of the posterior common bile duct wall in its intrapancreatic part at posterior medial side and formation of Roux-en-Y pancreatojejunoduodenostomy effectively stops abdominal pain, pancreatic and biliary obstruction, restores physiologic passage of the bile and pancreatic juice through the duodenum. It is a saving parenchyma technique and a function restoring operation.

\section{CP-C7 \\ Annular Pancreas as a Cause of Duodenal Obstruction in an Elderly Man}

\section{S. Kumar}

Department of Surgical Gastroentrology, Gateway Clinics, 43, West Periyasamy Road, RS Puram, Coimbatore, India

Introduction: Annular pancreas was first described by Tiedemann in 1818. It presents clinically as duodenal obstruction at the neonatal age group. We present a case of annular pancreas who presented at the age of 63 years. Methods: We present a patient who presented with indigestion, bloating and vomiting on and off since 5 years. His upper GI scopy showed obstruction at D2 with food stasis. CT scan also showed features suggestive of duodenal obstruction 
possibly by annular pancreas. Patient underwent laparoscopic RouxEn-Y DuodenoJejunostomy. Results: Patient improved symptomatically. Post operative upper GI scopy showed no stasis or obstruction and the patient is doing well. Conclusion: Annular pancreas causing duodenal obstruction is very uncommon at old age. This has to be considered as a cause for duodenal obstruction if clinical findings are in favour of it, irrespective of the age.

\section{CP-C8 \\ A Case of Hemosuccus Pancreaticus Accompanied by Autoimmune Pancreatitis}

H. Tanaka

Nagoya Medical Center, Nagoya, Japan

A 46-year-old woman had been followed up for pancreatic cysts for years. We recommend her to undergo operation, but she had declined. She developed sudden hematoemesis and was emergently admitted to our hospital. A CT scan showed that density of $20 \mathrm{~mm}$ cystic mas at the tail of the pancreas turn into high. A endoscopic ultrasound sonograhy showed iso echoic lesion. CT Angiography identified aneurysms at the distal portion of the splenic artery. We diagnosed it as hemosuccus pancreaticus and recommend her to have operation over and over again, but she didn't. The patient had intermittent hemorrhage every month with a shock condition, and a diagnosis of the rupture of aneurysm of the splenic artery was confirmed emergently. Intraoperative ultrasonography revealed a $20 \mathrm{~mm}$ low echoic mass at the body of the pancreas suspected as a hematoma. Distal pancreatectomy and splenectomy weres performed for the aneurysm of the splenic artery. The pancreas was diffusely edematous and compatible with chronic pancreatitis. It seemed that this might be caused by obstruction of main pancreatic duct due to blood from hematoma. Pathological examination confirmed pseudoaneurysm of the splenic artery communicated with the pancreaticduct: hemosuccus pancreaticus. Furthermore, it shows IgG4 positive plasmacyte, so we diagnosed it as hemosuccus pancreaticus accompanied by autoimmune pancreatitis. Postoperative ourse was uneventful without recurrence.

\section{CP-C9 \\ Single Center Experience in Endotherapy for Symptomatic Pediatric Pancreatic Duct Disease}

\section{A. Maydeo, M. Bapat, S. Bhandari, V. Dhir \\ Institute of Advanced Endoscopy, Mumbai, India}

Introduction: Treatment of symptomatic pancreatic duct disease in adults is well established. Similar data in Indian children is scant. Objective: To evaluate therapeutic outcomes in children with pancreatic stone disease. Design: Prospective study Setting: Single tertiary referral center. Patients: Consecutive patients between age 1 to 21 years presenting with symptomatic pancreatic duct disease.
Interventions: Pre procedure extracorporeal shock wave lithotripsy (ESWL) in patients with radio-opaque stones, pancreatic sphincterotomy, sphincteroplasty, stone removal and pancreatic duct stenting. Outcome measures: Safety of ESWL and pancreatic endotherapy in children, technical and therapeutic success in terms of pulverization of pancreatic duct stones post ESWL, pancreatic duct cannulation, stone removal, stent placement and pain relief. Results: Thirty one (17 girls, median age12 yrs) patients qualified to be included. Pathologies included 26 with pancreatic stones and strictures, pancreas divisum 3, one each of pancreatic pseudocyst and duct leak. ESWL was well tolerated in all. Those who underwent ESWL (median 9000 shocks) developed mild pain, responded well to oral analgesics. Stones could be removed in majority patients in average two sessions (technical success of ESWL (90\%). Pancreatic cannulation and stent placement was possible in all patients (therapeutic success 100\%) with average procedure time 25 minutes. None developed major procedure related complication. Significant pain relief was seen in $80 \%$ and 6 patients continued to have pain as per universal pain assessment tool. Conclusion: Pediatric pancreatic stone disease is a common pathology in India. ESWL appears to be safe and effective in children. Systematic pancreatic endotherapy seems to provide pain relief in children with pancreatic duct disease

\section{CP-C10 \\ Alcohol Influences the Clinical Profile and Course of Early Onset Chronic Pancreatitis}

\author{
V. Balakrishnan, A.B. Veena, K.R. Sundaram \\ Department of Gastroenterology, Amrita Institute of \\ Medical Sciences, Cochin, Kerala, India
}

Background: There is increasing alcoholism in India. Aim: To examine whether alcohol can influence the clinical profile and course of early- onset chronic pancreatitis (EOCP). Methods: 118 male patients (onset of first symptom -typical abdominal pain- at $\leq 30 \mathrm{ys}$ ). Mean age $29.30 \pm 10.509$ (8-57) ys. 55 were never-alcoholics (Group I), 63 imbibed alcohol in varying amounts (Group II). Of 40 smokers, 35 were alcoholic; 5 were never-alcoholic. No other risk factor. Clinical profile of pain, diabetes, calcification, childhood history of abdominal pain, family history of diabetes, pseudocyst, and pancreatic cancer was recorded, correlated with the alcohol (+ smoking) status. Alcohol intake graded as heavy (lifetime cumulative intake of $>400$ g.ys $(80$ g/day X 5ys) and moderate ( $\leq 400$ g.ys). Smokers were those smoking for $\geq 5$ ys. Results: Mean age of Grp I at onset of disease was $15.76 \pm 6.688(2-30)$ ys, while age of diagnosis was $20.38 \pm 5.939(5-48)$ ys [gap of 5 ys]. In Grp II, age at onset was $20.57 \pm 7.928(3-30)$ ys and age at diagnosis $32.95 \pm 7-866(15-48)$ ys (gap of 12 ys). All 118 patients had pain (equal in Grps I \&II). Diabetes present in 50/118 (42.4\%). More diabetics in Grp II (74\%) than Grp I $(26 \%)(p=0.0001)$. More diabetics among heavy drinkers than in moderate. Alcohol plus smoking increased risk of diabetes $(\mathrm{p}<0.0001)$. Childhood h/o abd pain higher in Grp I than in heavy alcoholics $(p=0.016)$ and moderate alcoholics (? genetic influence) $(p=0.001)$. Smoking was higher in GrpII (87.5\%) than in Grp I $(12.5 \%)(\mathrm{p}<0.001)$. No patient had cancer. Conclusions: Alcohol, even moderate drinking influences the clinical profile and course of EOCP-delayed onset, slower progression, delayed diagnosis and more diabetes. Alcohol, 
especially heavy, raises the risk for diabetes in CP. The risk is further increased by alcohol plus smoking.

\section{CP-C11}

\section{Orocecal Transit Time in Patients of Chronic Pancreatitis}

\author{
S.V. Rana, D.K. Bhasin, S.S. Rana, R.P. Ola, S. Sharma, \\ K. Singh
}

Department of Gastroenterology, Postgraduate Institute of Medical Education and Research, Chandigarh, India

Background: The exact mechanisms responsible for bowel disturbances in chronic pancreatitis (CP) are still unknown. Small bowel motor abnormalities may be involved in this pathological condition. However, there is no study addressing small bowel transit in patients of chronic pancreatitis. Aim: To measure the oro-cecal transit time (OCTT) in patients of chronic pancreatitis. Methods: In this study, 30 patients of chronic pancreatitis with age range of 21 to 65 years (group I) and 30 age and sex matched apparently healthy controls (group II) were enrolled. Orocecal transit time was measured by using non-invasive technique, lactulose hydrogen breath test. Results: Out of 30 patients of CP, 23 were males and 7 females while in controls, 22 males and 8 females. Mean $\pm \mathrm{SD}$ of age in $\mathrm{CP}$ was $43.6 \pm 22.1$ years while in controls $44.5 \pm 21.6$ years. Oro-cecal transit time in chronic pancreatitis was significantly delayed $(<0.01)$ $139.8 \pm 44.8$ minutes (Mean $\pm \mathrm{SD}$ ) as compared with $95.6 \pm 25.1$ minutes in apparently healthy controls. Out of 30 patients of chronic pancreatitis 4 patients were diabetics while in controls there was no patient with diabetes. OCTT was $>95$ minutes in 22 patients, out of which $2(9 \%)$ were diabetics. On the other hand, OCTT was $<95$ minutes in 8 patients of whom 2 were diabetics. OCTT in diabetic and non diabetics was (Mean \pm SD) $108.7 \pm 18.2$ and $145.2 \pm 43.5$ minutes respectively. Conclusion: Prolonged oro-cecal transit time in chronic pancreatitis observed in present study needs to be investigated further.

\section{CP-C12}

\section{Pancreatic Function and Clinical Correlation in Chronic Pancreatitis}

\author{
S.V. Daga, N. Bheerappa, R.A. Sastry \\ Department of Surgical Gastroenterology, Nizam's \\ Institute of Medical Sciences, Hyderabad, India
}

Background: Assessment of exocrine pancreatic function is not routine in chronic pancreatitis. Stool elastase is non-invasive test of choice for assessment of exocrine pancreatic function. However no studies of clinical correlation with stool elastase are reported. Aim: 1. To assess exocrine pancreatic function in chronic pancreatitis and draw a clinical correlation. 2. Evaluate the effect of enzyme supplement in patients of chronic pancreatitis with defined exocrine insufficiency. Methods: This is a prospective study done from June
2007 to December 2009. In diagnosed cases of chronic pancreatitis exocrine and endocrine pancreatic functions were assessed. Exocrine function was graded using using stool elastase and those defined to have insufficiency were treated with enzyme supplement. Endocrine function was assed by fasting and post meal blood sugar level and c-peptide. Results: 58 patients with chronic pancreatitis were evaluated. Severe exocrine insufficiency was seen in $47(81 \%)$, moderate insufficiency in $8(13.8 \%)$ and normal function was seen in $3(5.2 \%)$. All patients with steatorrhea had severe exocrine insufficiency. Mean stool elastase in patients with steatorrhea $(59.9 \pm 57.8)$ and without steatorrhea $(95.1 \pm 114.7)$ was less but difference was not significant $(\mathrm{p}=0.132)$. There was no difference in mean stool elastase in patients with weight loss $(89.4 \pm 111.8)$ and without weight loss was $(61.6 \pm 49.2)(\mathrm{p}=0.198)$. Patients treated with pancreatic supplement showed weight gain of $3.04 \pm 2.153 \mathrm{~kg}$ during follow up of 6-12 month. Conclusion: Stool elastase is helpful in diagnosis of preclinical exocrine insufficiency. This study gives the basis for further randomized study for assessing role of enzyme supplement in treatment of exocrine insufficiency diagnosed using stool elastase.

\section{CP-C13 \\ Clinical Presentation and Long Term Outcome of Endoscopic Therapy in Patients with Symptomatic Chronic Calcific and Non Calcific Pancreatitis Associated with Pancreas Divisum \\ D.K. Bhasin, S.S. Rana, R.S. Sidhu, B. Nagi, B.R. Thapa, \\ U. Poddar, R. Gupta, S.K. Sinha, K. Singh \\ Departments of Gastroenterology and Surgery, Postgraduate Institute of Medical Education and \\ Research, Chandigarh; Delhi Hospital, Bhatinda; Sanjay \\ Gandhi Postgraduate Institute of Medical Sciences, \\ Lucknow, India}

Background/Aims: The results of endoscopic drainage in pancreas divisum (PD) with chronic pancreatitis $(\mathrm{CP})$ have been debatable. We report clinical presentation and long term results of endoscopic therapy in 42 patients of calcific (15)/non calcific CP (27) with PD. Methods: Between 1996 and 2009, 42 patients were treated endoscopically. Patients were considered to have clinical success if they had resolution of symptoms and did not require surgery. The stents were exchanged 'on demand' or after 1 year and definite stent removal was attempted on basis of clinical and ERCP findings. Results: All presented with abdominal pain, had complete PD and symptoms were present for $32.9 \pm 42.4$ months. Pseudocyst, diabetes pancreatic pleural effusion, pancreatic ascites, and steatorrhea were seen in $8(19.1 \%), 5(11.9 \%), 2(4.7 \%), 1(2.3 \%)$ and $1(2.3 \%)$ patient respectively. All 5 patients with diabetes had CCP and there was no significant difference in the frequency of other complications between calcific and non-calcific CP. Twenty one patients each had moderate and severe changes as per Cambridge classification. Ductal calculi and strictures were noted in 3 and 2 patients respectively. Deep cannulation could not be achieved in 1 patient and was treated surgically. In 41 patients, an endoprosthesis $(5 / 7 \mathrm{~F})$ was successfully placed in dorsal duct. Minor papillotomy was done in 5 patients and ESWL in 2 
patients. Following stenting, $40(97.5 \%)$ patients had successful outcome. The mean number of stenting sessions required for success were $2.0 \pm 0.9$. There was no difference in outcome in calcific or noncalcific group. One patient each had mild post ERCP pancreatitis, in ward migration of stent and precipitation of diabetic ketoacidosis as complication of endoscopic therapy. The patients were followed up for $50.7 \pm 32.7$ (range $2-154$ ) months. Twelve (29.2\%) required restenting and none of these patients required surgery. Conclusion: In patients with both chronic calcific as well as non-calcific pancreatitis associated with PD, endoscopic dorsal duct drainage is safe and effective treatment modality for relief of pain with good long-term response.

\section{CP-C14 \\ Experience of Endoscopic Management for Relief of Pain in Tropical Pancreatitis in Children}

\author{
M. Patil, H. Devarbhavi, Prasanna K.S., D. Karanth \\ St John's Medical College, Bangalore, Karnataka, India
}

Background: Tropical pancreatitis frequently presents with intractable abdominal pain. Surgical treatment is associated with morbidity and mortality. Data on therapeutic endoscopic treatment for relief of pain in tropical pancreatitis in children are few. Aim: To determine the results of and present our experience of therapeutic ERCP for the relief of pain in tropical pancreatitis in children. Methods: Children below 18 years of age were included in the study. Tropical pancreatitis was defined by the presence of calcifications and' or ductal changes on US, CT, or on ERCP with other causes of chronic pancreatitis were excluded. Twenty patients of tropical pancreatitis with persistent severe pain not responding to medical line of treatment were in included in the study from January 2007 to April 2010. In all eligible patients, therapeutic ERCP in the form of pancreatic sphincterotomy with or without stent placement of pancreatic duct and dilation of the pancreatic stricture was carried out. Stent exchange was carried out on demand. All patients received antibiotics during and for 5 days after the procedure. Results: Mean age of onset of pain was $11 \pm 2.5$ years. Successful therapeutic ERCP was possible in 15 patients ( 8 male). Pancreatic sphincterotomy and stent placement of pancreatic duct was successful in 11 patients $(73.3 \%)$, in the rest (4) sphincterotomy alone was possible. Over a mean follow-up period of 18 months (range 8 to 26) abdominal pain improved in 9 of $11(81.8 \%)$ patients who underwent pancreatic sphincterotomy and stent placement and 2 out of 4 patients who had sphincterotomy alone. In 7 of 11 (63.6\%) patients who underwent sphincterotomy and stent placement had complete relief of abdominal pain and they were off the analgesics. The post ERCP complication rate was $20 \%$ including mild pancreatitis in 2 patients requiring more than 24 hours of hospitalization and sepsis in 1 patient requiring removal of the stent. Conclusion: Therapeutic ERCP offers short-term improvement in the pain in children with tropical pancreatitis, especially in those who underwent a successful sphincterotomy/ stenting.

\section{CP-C15 \\ Multidisciplinary Management and Outcome of Bleeding Pseudoaneurysm Associated with Chronic Pancreatitis}

V. Vimalraj, R. Sukumar, P. Senthilkumar, E. Selvakumar,
D. Jyothibasu, S. Jeswanth, P. Ravichandran, R. Surendran

Department of Surgical Gastroenterology and

Interventional Radiology, Stanley Medical College,

Chennai, India

Background: In patients with chronic pancreatitis, an actively bleeding pseudoaneurysm can be life-threatening. Optimal treatment of bleeding peripancreatic pseudoaneurysm in the setting of chronic pancreatitis remains controversial. This study reports our experience in managing of bleeding pseudoaneurysms associated with chronic pancreatitis. Methods: We reviewed our experience with management (both operative as well as angiographic embolization) of 37 patients with bleeding pseudoaneurysms [presenting as hemosuccus pancreaticus] complicating histologically documented chronic pancreatitis between January1997 and June 2010. Diagnosis of hemosuccus pancreaticus was based on clinical presentation, preoperative endoscopic and radiographic imaging, operative findings, and pathologic evaluation. Results: Mean patient age was 33 years $(8-55$ years). Fifteen patients had chronic alcoholic pancreatitis, 22 had tropical pancreatitis. Selective arterial embolization was attempted in 27 of $37(72 \%)$ patients and was successful in 19 of the $27(70 \%)$. Eighteen of 37 (48\%) patients required surgery to control bleeding after the failure of arterial embolization in 8 and in an emergent setting in 10 patients. Procedures included distal pancreatectomy and splenectomy, central pancreatectomy, intracystic ligation of the blood vessel in 10, 2 and 6 patients, respectively. There were no deaths. Length of follow-up ranged from 6 months to 12 years. Conclusion: Upper gastrointestinal bleeding in a patient with a history of chronic pancreatitis could be caused by HP. Diagnosis is based on investigations that should be performed in all patients, preferably during a period of active bleeding. Therapeutic options consist of selective embolization and surgery. Endovascular treatment can control unstable haemodynamics and can be sufficient in some cases. However, in patients with persistent unstable haemodynamics, recurrent bleeding or failed embolization, surgery is required.

\section{CP-C16 \\ Eosinophilia as an Independent Marker of Complicated Chronic Pancreatitis}

V. Venkatarami Reddy, R.V. Rao

Global Hospitals, Hyderabad, India

Background: Eosinophilia $\left(>0.6 \times 10^{9} / \mathrm{L}\right)$ is seen in patients with chronic pancreatitis (CP). But the clinical significance and the causes of eosinophilia are not well understood. Aim: To analyze inflammatory and malignant complications in CP patients, and assess the significance of eosinophilia. Methods: All the patients diagnosed to have CP during the period March 09 to June 10 were included in the study. We have prospectively analyzed patients for eosinophilia and 
any complicated presentation. Patients with parasitic infestations and allergic disorders were excluded. Results: There were 11 cases $(21.2 \%)$ of $\mathrm{CP}$ with eosinophilia among 52 patients. Average Eosinophilic count in patients with eosinophilia was $1.82 \times 10^{9} / \mathrm{L}$. The mean age was $31.3 \mathrm{yrs}$. The male to female ratio was $4.5: 1$. Eosinophilia did not differ among various etiology. The pain scores $(\mathrm{p}=0.55)$, exocrine insufficiency $(p=0.93)$, endocrine insufficiency $(p=0.39)$, ductal diameter $(p=0.79)$, intraductal pressure $(p=0.11)$, did not differ significantly in patients with and without eosinophilia. Enlargement of the gland was more in patients with eosinophilia $(\mathrm{p}=0.01)$. The incidence of complications were more in patients with eosinophilia $(p=<0.001) .18(34.6 \%)$ of 52 CP patients presented with complicated disease, of which 15 were inflammatory and 3 were malignant. The incidence of inflammatory complications - Pseudocyst, Biliary obstruction, Duodenal obstruction, SV or PV thrombosis, Pleural effusion and Ascites were 13.5\%, 9.6\%, 1.9\%, 7.7\%, 3.8\% and 3.8\% respectively. $9(81.8 \%)$ of 11 patients with eosinophilia had complicated presentation, of which 5 had more than one complication. The incidence of pseudocyst $(p=0.01)$, Biliary obstruction $(p=0.03)$, Duodenal obstruction $(p=0.05), S V$ or PV thrombosis $(p=0.006)$, Pleural effusion $(p=0.005)$ and ascites $(p=0.005)$ were significantly high in patients with eosinophilia. None of the patients with malignancy had eosinophilia. Conclusions: Eosinophilia in CP is not unusual. Patients with eosinophilia mostly present with inflammatory complications. Further studies are required to assess outcomes of treating eosinophilia in $\mathrm{CP}$.

\section{CP-C17 \\ Predictors of Malignancy in Pancreatic Head Mass with Chronic Calcific Pancreatitis - A Clinico-pathological Correlative Study}

R. Subhash, R.S. Sindhu, T.U. Shabeerali, M.L. Arunkumar, V.A. lyoob, R. Rajan, B. Natesh

Department of Surgical Gastroenterology, Medical College, Thiruvananthapuram, India

Background: Pancreatic head mass (PHM) in chronic calcific pancreatitis $(\mathrm{CCP})$ is a matter of diagnostic and therapeutic dilemma. Aim: To predict the nature of PHM in CCP by correlating the clinical and investigational details of patients who underwent surgery with histopathology and follow-up. Methods: All CCP patients with PHM over a period of 5 years (2004-2009), who underwent some form of surgical procedure and had a histopathological diagnosis were retrospectively analysed from a prospectively collected database. All patients were then followed up. Chi-square test was used to assess the relation with categorical variables and non-parametric Mann-Whitney test for continuous variables. Odds Ratio based on univariate and multiple logistic regression analysis investigated the effects of variables that were significantly associated with malignancy in PHM with CCP using SPSS.V.16.0. Results: Out of 94 patients 40 had histologically proven malignancy in PHM. On follow-up (average follow-up 28months) 4 patients with benign histology later presented with features of malignancy. Logistic regression analysis showed that a change in pain pattern Odds Ratio $(95 \%$ Confidence Interval)-10.80 (3.60-32.38) $\}$, Obstructive jaundice $\{72.00(14.97-$ $346.24)\}$, Diabetes with sudden change in insulin requirement
$\{20.13(6.11-66.29)\}$, Gastric outlet obstruction $\{3.63(1.38-9.55)\}$, serum bilirubin $>4.9 \mathrm{mg} / \mathrm{dl}$, serum alkaline phosphate $>435 \mathrm{IU} / \mathrm{L}$, and serum CA 19-9>252 U/ml are the major factors associated with the malignancy in PHM with CCP. Conclusion: A good clinical and investigational analysis can predict the nature of pancreatic head mass in chronic calcific pancreatitis in majority of cases.

\section{CP-C18 \\ Pancreatic Head Mass in Chronic Pancreatitis Is Mostly Inflammatory in Alcoholic Pancreatitis and Malignant in Idiopathic Pancreatitis}

\author{
S. Midha, S. Shalimar, A. Kumar, P.K. Garg \\ Department of Gastroenterology, All India Institute of \\ Medical Sciences, New Delhi, India
}

Background: Pancreatic head mass in patients with chronic pancreatitis (CP) is a diagnostic dilemma with malignancy being a major concern. Objective: To determine the etiology of pancreatic head mass in patients with CP. Methods: All consecutive patients with CP who had a pancreatic head mass on imaging (computed tomography scan) formed the study group. They were subjected to clinical evaluation and imaging studies including PET-CT scan and endosonography (EUS). The diagnosis of malignancy versus inflammatory mass was confirmed on histo/cytopathology and clinical criteria. Patients were treated with a standard protocol. Results: Of 402 patients with $\mathrm{CP}, 57$ presented with a pancreatic head mass. Their mean age was 44.5 years; 49 were males. Etiology of CP was alcohol in $29(51 \%)$, idiopathic in $27(47 \%)$ and hereditary in one patient. 29 of 57 patients had associated biliary obstruction. Of the 57 patients, 6 were found to have malignancy, 32 had inflammatory mass, EUS ruled out any mass in 15 and 4 patients were lost to follow up. The mean age of 6 patients with malignancy was 47.5 years and the duration of CP was 13.93 years. Of these 6 patients with malignancy and $\mathrm{CP}$, the etiology of $\mathrm{CP}$ was idiopathic in 5 and hereditary in 1 patient. All the 6 patients had pancreatic calcification, 5 had diabetes, and 3 had biliary obstruction. Of 29 patients with alcoholic CP, 26 had an inflammatory mass and no mass was confirmed in 3 patients. Of 27 patients with idiopathic CP, 5 had malignancy, 3 had inflammatory mass, and no mass was confirmed in the remaining 19 patients. Conclusion: Pancreatic head mass was inflammatory in nature in alcoholic $\mathrm{CP}$ while the mass was often malignant in idiopathic $\mathrm{CP}$. 


\section{CP-C19 \\ Long-term Pain Relief with Optimized Medical Therapy Including Antioxidants in Chronic Pancreatitis}

S. Shalimar, S. Midha, P. Bhardwaj, P.K. Garg

Department of Gastroenterology, All India Institute of

Medical Sciences, New Delhi, India

Background: Pain due to chronic pancreatitis (CP) is difficult to treat. We have shown that antioxidants relieve pain in the shortterm. Objective: To study the long-term result of antioxidants for pain relief. Methods: All consecutive patients with $\mathrm{CP}$ were studied prospectively. They were treated medically with well-balanced diet, pancreatic enzymes, and antioxidants $(9000 \mathrm{IU}$ b-carotene, $0.54 \mathrm{~g}$ vitamin C, 270IU vitamin E, 600mg organic selenium, and $2 \mathrm{~g}$ methionine) for 6 months-1 year. Endoscopic therapy and/or surgery were offered if medical therapy failed. The response to therapy was evaluated with a composite pain score and the need for hospitalization. Pain relief was the primary outcome measure. Results: 226 patients (178 males) with CP were included. The etiology was idiopathic in $167(73.9 \%)$, alcohol in $53(23.5 \%)$ and miscellaneous in $6(2.6 \%)$ patients. The mean age at onset was 22.31 years for idiopathic and 36.86 years for alcohol CP. The mean follow-up was $8.71 \pm 6.42$ years. $208(92 \%)$ had abdominal pain. Significant pain relief was seen in $81.7 \%$ of patients: $45.6 \%$ with medical therapy, $11.1 \%$ spontaneously, $18.3 \%$ with endotheray and $6.7 \%$ with surgery. The mean pain score decreased from $6.33 \pm 1.94$ to $3.09 \pm 3.34$ ( $\mathrm{p}<0.001$ ). Of $208,193,151$, and 80 patients followed up for 1, 3, 5 and 10 years, 50.5\%, 53.4\%, $58.3 \%$ and $67.5 \%$ became pain free respectively. Steatorrhoea and diabetes developed in $11.1 \%$ and $35.4 \%$ respectively. Portal vein thrombosis, biliary stricture and pseudocyst were seen in $4 \%, 8.8 \%$ and $21.7 \%$ respectively. Carcinoma pancreas developed in $4(1.7 \%)$ patients. Conclusion: Optimized medical therapy with antioxidants results in long lasting pain relief in over $50 \%$ of patients and in $80 \%$ of patients with additional intervention.

\section{CP-C20 \\ Surgical Management of Patients with Non- alcoholic Chronic Pancreatitis in a North Indian Tertiary Care Hospital: 25 Years Experience}

S. Kaistha, S. Pal, J. George, P. Sahni, N.R. Dash, P.K. Garg, T.K. Chattopadhyay

Department of GI Surgery, All India Institute of Medical Sciences, New Delhi, India

Background: Reports detailing the long term experience of surgery for non-alcoholic chronic pancreatitis (NACP) are sparse. Methods: Data from a prospectively maintained database of all patients with NACP operated between 1985 and 2010 was analyzed with regard to demographics, indications for surgery, type of surgery, postoperative outcome, pain relief and follow up. Results: Of 242 patients operated for chronic pancreatitis, 148 (61.2\%) underwent surgery for NACP. There were 89 males and the mean age was 36 years (range: 5-72 years). The indications for surgery were abdominal pain $(92 \%)$, biliary obstruction $(21.6 \%)$, pseudocyst drainage (17.6\%), left-sided portal hypertension (13.5\%), duodenal obstruction $(7.4 \%)$, and inflammatory head mass $(5.4 \%)$. Patients with dilated main pancreatic duct $(>5 \mathrm{~mm})$ underwent lateral pancreatojejunostomy (LPJ) + head coring $(97 ; 65.5 \%)$, others underwent resections such as pancreatoduodenectomy $(11 ; 7.4 \%)$, distal pancreatectomy $(5 ; 3.4 \%)$ and total pancreatectomy $(2 ; 1.4 \%)$. Four (2.7\%) patients died. Over a median follow up of 63 months (6-300 months) satisfactory pain relief was obtained in 121 patients $(84 \%)$. There were 7 late deaths on follow up (4 due to pancreatic cancer). Conclusions: Ductal drainage or resection could be safely offered to a majority $(77.7 \%)$ of the patients with NACP with lasting pain relief in $84 \%$. Late deaths may occur due to pancreatic cancer in some of these patients.

\section{CP-C21 \\ Long Term Experience with Surgery for Alcoholic Chronic Pancreatitis in a Tertiary Care Hospital from North India}

\section{S. Pal, S. Kaistha, J. George, N.R. Dash, P.K. Garg, P. Sahni, T.K. Chattopadhyay}

\section{Department of GI Surgery, All India Institute of Medical} Sciences, New Delhi, India

Background: There is a paucity of data with regard to the surgical management of patients with alcoholic chronic pancreatitis (ACP) from India. Methods: Data from prospectively filled records of all patients with ACP operated between 1985 and 2010 was analyzed with regard to demographics, indication for surgery, type of surgery, postoperative outcome, pain relief and follow up. Results: Of 242 patients with chronic pancreatitis undergoing surgery, 94 (38.8\%; only 1 woman) had ACP. Their mean age was 42 years (range: $25-65$ years). The main indication for surgery was pain which was present in all patients; in addition they had biliary obstruction (34\%), pseudocysts $(27.7 \%)$, left-sided portal hypertension (11.7\%), celiac axis pseudoaneurysms (10.6\%), inflammatory head mass (8.5\%) and duodenal obstruction $(3.2 \%)$. Patients with a dilated main pancreatic duct (>5 mm) underwent lateral pancreatojejunostomy (LPJ) + head coring $(47 ; 50 \%)$, others underwent resections [such as distal pancreatectomy $(9 ; 9.6 \%)$, pancreatoduodenectomy $(8 ; 8.5 \%)]$, pseudocyst drainage $(16 ; 17 \%)$ and aneurysmorrhaphy $(7 ; 7.4 \%)$. Five $(5.3 \%)$ patients died. Over a median follow up of 60 months (6-280 months) satisfactory pain relief was obtained in 73 patients $(82 \%)$. There were 2 late deaths on follow up (1 due to pancreatic cancer). Conclusions: About two-thirds of the patients with ACP were suitable for ductal drainage or resectional procedures and, in the remaining surgery was tailored to treat the complications of ACP. Lasting and satisfactory pain relief was achieved in $82 \%$ of the patients with acceptable operative mortality. 


\section{CP-C22 \\ Is Idiopathic Chronic Pancreatitis in India Same as the West?}

\author{
J. Augustine, A. Chacko, A.K. Dutta \\ Department of Gastrointestinal Sciences, Christian \\ Medical College, Vellore, Tamil Nadu, India
}

Aim: To study the demographic profile of Idiopathic chronic pancreatitis (ICP) in a cohort of patients attending tertiary care medical gastroenterology department. Methods: Data on consecutive patients diagnosed to have chronic pancreatitis based on imaging studies (USG, CECT, MRI, ERCP or EUS) attending gastroenterology OPD at CMC Vellore between January 2008 to May 2010 were analysed retrospectively. Patients were divided into groups as follows: Alcoholic chronic pancreatitis (ACP), Early-onset ( $\leq 30 \mathrm{yrs})$ idiopathic chronic pancreatitis (EICP) and Late-onset (>30yrs) idiopathic chronic pancreatitis (LICP). Statistical analysis was done using chi-square test and Mann-Whitney test. Results: Of 255 patients, 74 (29\%) were females and $181(71 \%)$ were males. Majority of patients $211(82.7 \%)$ were from Eastern India. ACP was noted in $48(18.8 \%)$, EICP in 97 (38\%) and LICP in $110(43.1 \%)$ of patients. Abdominal pain was more common in EICP as compared to LICP $(95.5 \%$ vs $83.3 \%, \mathrm{p}=0.01$ ). The prevalence of pancreatic calcification (parenchymal and ductal), diabetes, and steatorrhoea in EICP was $55.7 \%$, $36.5 \%$ and $43.3 \%$ respectively whereas in LICP, the prevalence was $57.3 \%, 67.3 \%$ and $64.5 \%$ respectively. Diabetes and Steatorrhoea were more common in LICP as compared to EICP $(p=0.01)$. Conclusion: The early-onset idiopathic chronic pancreatitis (EICP) in India differs from the West as evidenced by a higher prevalence of diabetes, steatorrhoea and pancreatic calculi.

\section{CP-C23 \\ Does Acute Pancreatitis Lead to Chronic Calcific Pancreatitis in the Tropics?}

\section{Thomas, F. Jose, K. Sunil Kumar}

Dept of Gastroenterology, Calicut Medical College, Kerala, India

Introduction: The natural history and aetiology of idiopathic calcific pancreatitis in the tropics is not well established. Aim: To study whether acute pancreatitis (AP) or recurrent acute pancreatitis (RAP) leads to development of chronic pancreatitis (CP) on long term follow-up. Methods: In a retrospective study done in 2009, 112 patients diagnosed to have AP or RAP, ten or more years earlier were re-evaluated. Patients were identified from the medical records of the Dept. of Gastroenterology of Calicut Medical College. Patients were contacted through mail and later by telephone. All patients who came for follow up were interviewed for recurrence of pain, symptoms of steatorrhoea or history of detection of diabetes mellitus. All available health records until the day of follow-up were scrutinised. Those who were asymptomatic after first attack were requested to have post prandial blood glucose estimation and abdominal ultrasonogram. Diagnostic criteria for AP were: abdominal pain, elevated amylase, radiographic evidence of acute pancreatitis on ultrasound scan or CT scan. Criteria for RAP: one episode of confirmed AP with recurrence of symptoms and confirmation by serum amylase/lipase study and radiographic evidence of acute pancreatitis. Criteria for CP: abdominal pain, CT or sonological evidence of CP with or without calcification. Results: Of the total 167 patients with AP or RAP, only 112 patients could be traced after 10 years. Male: female ratio $=4: 3$, median age at the onset of pain: 23 years (range 8- 58). AP: $n=71$ $(63 \%)$ and RAP: $n=41(37 \%)$. The cause of pancreatitis was: alcohol: 23, gall stone related: 14 , viral: 9 , drug induced: 7 , unknown: 59 . Mean follow-up period was 12.2 years (range 10-26 years). 2/71(3\%) in AP group (both alcohol) developed CP where as 9/41 patients $(22 \%, 3$ alcohol) in RAP group developed CP during the follow up period $(\mathrm{p}<.0 .5)$. The cause for RAP could not be ascertained in 6 out of 9 patients (67\%) who developed CP. Conclusion: Recurrent idiopathic acute pancreatitis is a significant risk factor for development of chronic pancreatitis in the tropics.

\section{CP-C24}

\section{Natural History of Tropical Pancreatitis (TP):} A Prospective Study

\section{K.T. Shenoy, L.K. Balakumaran \\ Sree Gokulam Medical College and Research Foundation Thiruvananthapuram, Kerala}

Objective: To assess the long term sequele of TP. Design: Prospective. Setting: Tertiary centre. Methods: We recruited 1480 subjects with TP during the period 1980 January to March 2006 at the Govt. Medical College, Trivandrum and evaluated them periodically for sequele and complications such as pancreatic fluid collection, cholestasis and malignancy. Data were censored in January 2010. Diabetes, abdominal pain, alcohol and tobacco use were noted. Analysis: Survival curves using Kaplan Meier Product limit method and Cox Proportional Hazard regression. Results: 96\% had calcific pancreatitis, Median follow up was 242 months. 164 subjects developed pancreatic malignancy and the age was $45.5 \pm 12.4$ compared to those without malignancy ( $34.2 \pm 12.082$ years); majority of the pancreatic carcinoma had hepatic and/or distant metastasis and inoperable. 12 had pancreatic ascites; 8 pancreatic abscess. KM survival probability were $0.54(95 \%$ CI $0.41-0.66)$ and 0.12 at 9 months (95\% CI 0.06-0.22). Diabetes duration and abdominal pain did not affect survival. Conclusion: TP is a premalignant disease and pancreatitic malignancy present at an advanced stage with poor survival. 


\section{CP-C25}

\section{Profile of Childhood Chronic Pancreatitis in Coastal Eastern India}

\author{
H.S. Das, B. Misra, M.K. Panigrahi, S.K. Kar, C. Panda, \\ S.P. Singh \\ Department of Gastroenterology, S.C.B. Medical College, \\ Cuttack, Orissa, India
}

Background: Childhood chronic pancreatitis is not common in coastal eastern India. Aim: To undertake a retrospective study of the clinical profile, etiology and response to treatment of the cases of chronic pancreatitis in paediatric population. Methods: The retrospective study is an analysis of consecutive patients with chronic pancreatitis $(\mathrm{CP})$ under the age of $15 y$ ears who attended the Department of Gastroenterology over a period of 2 years. Results: Total no of cases studied were 10 in number of those $60 \%$ were males while $40 \%$ were females with sex ratio (M:F ) of 3:2. The age at presentation ranged between $8-14$ years with a mean age of 11 years. The most common presentation was pain abdomen seen in $80 \%$ cases, followed by steatorrhoea in $30 \%$, and ascites in $40 \%$ cases. Sixty percent of children had multiple calculi. One patient had pleural effusion with pancreatic ascites. A patient of sickle cell disease with uncontrollable pain and ascites improved following surgery and another case of autoimmune pancreatitis is on steroid. One patient was diabetic at presentation and now on insulin. Conclusion: Childhood pancreatitis accounted for $6 \%$ cases of all cases of chronic pancreatitis in our study. Most of them were calcific in nature. Pancreatic ascites was more prevalent in childhood pancreatitis as compared to adults.

\section{CP-C26}

\section{Autoimmune Pancreatitis in Pancreatic Resections for Chronic Pancreatitis or Pancreatic Masses - An Experience of Tertiary Centre from North India}

\section{R. Nada, D.K. Bhasin, S. Rana, R. Gupta, L. Kaman, T.D. Yadav, K. Joshi}

Departments of Pathology, Gastroenterology and Surgery, Postgraduate Institute of Medical Education and Research, India

Background: Autoimmune pancreatitis is a form of pancreatitis which has specific diagnostic histomorphology and immunohistology that distinguishes it from other forms of chronic pancreatitis and can be treated medically. Aim: This retrospective study was done to identify cases of autoimmune pancreatitis in specimens of pancreatic resections done for painful chronic pancreatitis or pancreatic masses. Methods: Hematoxylin and eosin stained slides of pancreatic resections over the period of 5 years from 2006 -2010 were reviewed. Immunohistochemistry for IgG4 was done in cases suggestive of AIP. Ten cases of pancreatic carcinoma and other cases of chronic pancreatitis with plasma cells (26) were taken as controls. During histomorphological analysis pathologists were blinded for the clinical details and later clinico-pathological correlation was done. Cases were cate- gorized as idiopathic pancreatitis, alcoholic pancreatitis and autoimmune pancreatitis based on clinical, hematological, radiological and/ or histological details. Results: Sixty one cases of chronic pancreatitis and 56 cases of pancreatic masses underwent surgery over last 5 years. Out of 61 cases of chronic pancreatitis, 53(86.68\%) cases had idiopathic chronic pancreatitis, 7 (11.47\%) had alcohol related pancreatitis and one patient $(1.63 \%)$ had histological features of autoimmune pancreatitis. Out of 56 cases of pancreatic masses, $47(83.92 \%)$ had malignancy, $7(12.50 \%)$ had focal inflammatory masses and $2(3.57 \%)$ had histomorphology of autoimmune pancreatitis. Of these 3 patients of autoimmune pancreatitis, there were 2 females and 1 male $(42,55$, and 60 years). Histomorphology of autoimmune pancreatitis was characteristic of lymphoplasmacytic sclerosing pancreatitis. There was duct centric plasma cell rich inflammation and the plasma cells were IgG4 positive ( $>10$ high power field). Venulitis and arterial oblitrative changes were seen in all these cases of AIP. Conclusion: In our study $3.57 \%$ of patients with pancreatic masses and $1.63 \%$ of patients with chronic pancreatitis who had undergone surgery had evidence of autoimmune pancreatitis.

\author{
CP-C27 \\ Profile of Chronic Pancreatitis in Coastal \\ Eastern India \\ B. Misra, S.K. Kar, M.K. Panigrahi, H.S. Das, C. Panda, \\ S.P. Singh \\ S.C.B. Medical College, Cuttack, Orissa, India
}

Background: Chronic pancreatitis (CP) is not uncommon in coastal eastern India. Aim: To undertake a retrospective study of the clinical profile, etiology and response to treatment of chronic pancreatitis in coastal eastern India. Methods: Retrospective study of patients with chronic pancreatitis presenting to the Department of Gastroenterology during the period 2006-2010. Results: Data of 150 patients of $\mathrm{CP}$ were studied. $67.33 \%$ were males with a sex ratio (M:F) of 2:1. The age of presentation was between $8-71$ years with a mean age of $35.70 \pm 13.08$. The most common presentation was pain abdomen found in $86.00 \%(129 / 150)$ cases, followed by 'oily leak' $37.33 \%(56 / 150)$, diarrhoea $18 \%(27 / 150)$, and ascites in $8 \%(12 / 150)$ of cases. Out of 150 cases, $22 \%(33 / 150)$ were diabetic at presentation. Multiple ductal and parenchymal calcifications were seen in $80 \%(120 / 150)$ of cases and pseudocyst in (13/150) 8.66\%, while duodenal obstruction was observed on gastroscopy in $6.66 \%(10 / 150)$. Twenty one percent (32/150) were chronic alcoholics and three cases (2\%) had family history of pancreatitis involving eight persons; one case was autoimmune in nature. Most patients responded satisfactorily to conservative management. Only 22 cases (14.66\%) needed surgery and all of them benefited by surgery. Conclusions: Idiopathic chronic pancreatitis is the most common form of CP in coastal eastern India followed by alcoholic pancreatitis which accounts for one fifth of all CP cases. Majority of these patients presented with abdominal pain, and only a third presented with 'oily leak' while diabetes mellitus was present in about one fourth. Most patients responded satisfactorily to conservative management. 


\section{CP-C28 \\ Chronic Pancreatitis Amongst Children in India}

\author{
S.D. Chowdhury, J. Augustine, A. Chacko \\ Christian Medical College, Vellore, India
}

Background: There is paucity of data regarding the clinical profile of chronic pancreatitis amongst children in India. The study was done with an aim to determine the etiology, clinical presentation and the complications of chronic pancreatitis amongst children. Methods: This was a retrospective study done at a tertiary care hospital in South India. Children diagnosed with chronic pancreatitis (on imaging study) who attended the pancreatic clinic, between January 2005 and December 2009 were recruited for the study. Results: Chronic pancreatitis was diagnosed in 73 children (boys: 51; girls: 22). The mean age of disease onset was 10 years and mean age at presentation was 12.3 years. Etiological evaluation revealed that the majority of patients $(92 \%)$ had Idiopathic chronic pancreatitis and $6.8 \%$ Tropical pancreatitis. One patient had a history of blunt abdominal trauma prior to the onset of the disease. All patients presented with severe recurrent abdominal pain. Calcification of the pancreas (ductal/acinar) was seen in $72 \%$ of the cases. Eight $(10.3 \%)$ patients had diabetes mellitus. There was poor glycaemic control (mean HbA1C - 10.8 SD-2). Disruptive complications in the form of pancreatic pseudocyst and pancreatic ascites were seen in $10.9 \%$ and $12.3 \%$ respectively. Thrombotic complication in the form of portal and or splenic vein thromboses was seen in $5.2 \%$ patients. None of the patients complained of steatorrhoea. Conclusion: This is one of the largest series of chronic pancreatitis in India. Idiopathic chronic pancreatitis is the predominant form of chronic pancreatitis in India. Chronic pancreatitis in India is an aggressive disease, with recurrent abdominal pain, early pancreatic calcifications, significant disruptive complications, and high incidence of diabetes mellitus and thrombotic complications.

\section{CP-C29}

\section{Short and Long Term Outcomes After Frey Procedure for Chronic Pancreatitis: A 15 Year, 400 Case Experience}

\author{
S. Rather, K.P. Manjuraj, M.M.S. Bedi, G. Singh, \\ B. Venugopal, H. Ramesh \\ Lakeshore Hospital, Cochin, Kerala, India
}

Background: Data regarding long term outcomes after surgery for chronic pancreatitis are lacking. Aim: Determine long term outcomes after Pancreaticojejunostomy with head coring (Frey procedure) for chronic pancreatitis over the period 1993-2008. Methods: 400 patients (257 male, 143 female, age range 8-71 years, median 42.5 years) underwent Frey procedure for intractable pain $(n=157)$, complications $(n=219)$, or asymptomatic pancreatic calculi $(n=24)$. Early outcomes studied were mortality and morbidity rates, and long term outcomes studied were pain relief, changes in exocrine/endocrine status (both subjective and objective), and quality of life. Factors affecting outcome were analysed by univariate and multivariate analysis. Minimum follow up was 1 year and maximum was 15 years (median 98 months). Results: 5 patients died, all in the period 1993-2000, (pancreatic leak with secondary hemorrhage $=2$, intra-abdominal sepsis secondary to leak $(n=1)$, Adult respiratory distress syndrome $n=1)$ and unexplained $(n=1)$. Postoperative complication rates occurred in $21 \%$ (intra abdominal collection, postoperative chest infection, fever, pancreatic leak, bleed, and wound related complications). 11 patients died due to intercurrent and related illnesses during the follow up. Pain relief occurred in 336 patients $(84 \%)$ when calculated at the end of 1 year follow up. At 5 years, the pain relief figure was $94 \% .43$ patients underwent reoperation/reinterventions. Endocrine status improved in $18 \%$, worsened in $16 \%$ and remained constant in $66 \%$ of survivors. On univariate analysis, acute presentation, alcohol dependence, residual head calculi, pancreatic head mass affected outcome. On multivariate analysis, residual head calculi was the only significant factor (OR 3.14, 95\% CI 1.09-6.87). On QOL assessments, 92\% had excellent or good quality of life. Conclusion: a) Surgery relieves pain and provides quality of life in over $90 \%$ of patients in the long term (against the myth that long term results are poor). b) pancreatic function is maintained following Frey procedure, c) a significant number of patients required postoperative interventions, or died due to both related and intercurrent illness.

\section{CP-C30 \\ Definitive Surgery in Chronic Pancreatitis During a Phase of Acute Exacerbation: Is It Safe?}

G. Singh, M.M.S. Bedi, B. Abraham, V. Lekha, B. Venugopal, H. Ramesh

Lakeshore Hospital, Cochin, India

Background: Chronic pancreatitis is a disease characterized by remissions and exacerbations. Surgery during acute exacerbation may be required if the symptom free intervals are short. Aim: Compare the outcomes for surgery during an acute episode with those during the interval phase. Design: Retrospective analysis of prospective data. Methods: Out of 201 patients operated for chronic pancreatitis during the period 2005-2009, 53 patients were in group 1(during the same admission of an acute episode with one of the following: a) high amylase; b) peripancreatic collection or peripancreatic fat stranding; c) raised $\mathrm{C}$ reactive protein, and d) fever and clinical sepsis), and 152 patients were in Group 2 (during the interval between episodes and none of the above). Outcomes such as mortality, morbidity, complications, hospital stay and early results such as pain relief were assessed. Results: Of 52 patients, 7 cases were abandoned after peripancreatic lavage and placement of drains, as there were dense adhesions and severe inflammation. In 4 cases, drainage of an infected collection was alone performed. 41 cases underwent the Frey procedure. There was no mortality. Complications occurred in 14 patients (2 patients pancreatic fistula, prolonged ileus in 6 patients, gastric stasis in 1 patient, intestinal obstruction in 2 patients, 1 case of Adult respiratory distress syndrome and 4 patients had major wound infections). Univariate analysis revealed that peripancreatic necrotic material, raised C Reactive protein above 40, and systemic sepsis predisposed to increased complications. In multivariate analysis, CRP levels above 40 correlated with complications, and dictated conservative surgery 
for draining pus only. Hospital stay and ICU stay was significantly prolonged among Group 1 versus Group 2. Conclusions: Surgery can be performed in patients with chronic pancreatitis during the acute attack. Patients with sepsis, peripancreatic necrosis, and high CRP over 40 may benefit from conservative measures.

\section{CP-C31 \\ Does Endocrine and Exocrine Pancreatic Function in Chronic Pancreatitis Improve After the Frey Procedure?}

\author{
S. Mahesh, M.M.S. Bedi, V. Lekha, H. Ramesh \\ Lakeshore Hospital and Research Center, Cochin, Kerala, \\ India
}

Background: There is paucity of data about the functional outcomes after Frey procedure in chronic pancreatitis. Aim: To study the endocrine and exocrine status before and after Frey procedure in patients with chronic pancreatitis. Methods: Patients operated during the period February 2008 and March 2010, and who were available for a minimum follow up of 1 year were included in the study. Exocrine function was assessed by fecal elastase levels before surgery and 1 year after surgery while endocrine function was assessed by fasting and stimulated $\mathrm{C}$ peptide levels and diabetic status before and 1 year after surgery. Results were expressed as means. Wilcoxon test was used to compare the preoperative and postoperative variables while means were compared by student paired t test using SPSS ver 17. A value $<0.05$ were considered significant. Results: There were 20 males and 11 female patients with mean age of 32.3 years. 5 patients had steatorrhoea before surgery and 7 were diabetic. Fecal elastase levels showed significant increase at 1 year after surgery $(p=0.021)$ while $c$ peptide levels basal as well as fasting did not show significant improvement after surgery. There was significant weight gain after surgery. Patients with fecal elastase levels less than 100 (defined as severe exocrine insufficiency) showed significant improvement in exocrine function after surgery at 1 year as compared with those with fecal elastase levels $>100(p=0.034)$. Conclusion: Surgery for chronic pancreatitis results in improvement in exocrine function while endocrine function does not show any improvement at 1 year of surgery. Maximum improvement in exocrine function was found in patients with severe exocrine insufficiency.

\section{CP-C32 \\ Head Masses in Chronic Pancreatitis: Which Factors Indicate Malignancy?}

M. Wani, M.M.S. Bedi, B. Venugopal, A. Venugopal,

S. Mahesh, V. Lekha, H. Ramesh

Lakeshore Hospital, Cochin, India

Background: Mass lesions in the head of pancreas in chronic pancreatitis present great diagnostic challenges. A satisfactory diagnostic test is not available. Aim: Analyze the preoperative parame- ters of patients with chronic pancreatitis and head masses to determine risk factors for malignancy; create a regression model; and validate it prospectively. Methods: Divide the dataset into two parts; perform univariate and multivariate analysis on factors correlating with malignancy, construct a regression equation, and calculate the coefficient on the second data set, and perform cross validation. Results: There were 145 patients with mass lesions in the head in chronic pancreatitis. 105 patients (consecutive) were included in the primary analysis. 53 had cancer (proven by biopsy) and 52 had benign disease (proven by length of follow up). Univariate analysis revealed more males in the cancer group, older age (median 50.5 versus 45 ), shorter symptom duration (1.5 months vs 4 months), more weight loss (48 out of 52 vs 22 out of 53 cases), higher bilirubin levels (6 mg vs 1.1) and CA 19-9 levels (median 68.3 vs 21). Multivariate analysis revealed that bilirubin levels, weight loss and high CA 19-9 levels were alone significant factors correlating with cancer.

\begin{tabular}{llll}
\hline Factor & Sensitivity & Specificity & Area under curve \\
\hline Bilirubin & 65.4 & 86.8 & 0.787 \\
Weight loss & 92.3 & 58.5 & 0.754 \\
CA 19-9 & 69.2 & 56.6 & 0.636 \\
Symptom duration & 71.2 & 64.2 & 0.708 \\
\hline
\end{tabular}

A regression equation was revalidated using the next 40 patients. Conclusion: High bilirubin levels, weight loss, and high CA 19-9 levels correlated with malignancy in head masses in chronic pancreatitis.

\section{CP-C33 \\ Stellate Cell Activation in Tropical Calcific Pancreatitis}

J. Cyriac, P. Augustine, P. Mahadevan, H. Ramesh,

A. Koshy

Departments of Gastroenterology, Pathology and GI

Surgery, Lakeshore Hospital, Cochin, Kerala, India

Background/Aims: Pancreatic stellate cell (PaSC) is known to be activated in pancreatic pathology of various etiologies. However, there is no published data on activation of PaSCs in tropical calcific pancreatitis. The present study was undertaken to estimate the proportion of activated stellate cells, in a semi-quantitative manner, in normal pancreas and pancreatic fibrosis due to, tropical calcific pancreatitis (TCP), alcoholic chronic pancreatitis (ACP) and pancreatic adenocarcinoma $(\mathrm{CaP})$. Methods: Expression of CD34, and alphasmooth muscle actin ( $\alpha$-SMA) was assessed by immunohistochemistry. Morphometry was performed by a point counting procedure and CD34 positive areas were excluded from $\alpha$-SMA positive areas for counting activated stellate cells. Student's T-test was used to compare the proportion of activated stellate cells among the four categories. Results: In all the disease conditions studied, namely, TCP $(17+12$, $\%, \mathrm{M}+\mathrm{SD}), \mathrm{ACP}(14+12)$ and $\mathrm{CaP}(23+13)$, there was highly significant increased percentage of activated pancreatic stellate cells (PaSCs) compared to normal $(2+4)$ pancreas $(\mathrm{p}<0.0002)$. The proportion of 
activated PaSCs in TCP was similar to that in cases of ACP and $\mathrm{CaP}$. Such activation has been documented for the first time in case of TCP while it is already known for the other causes. Conclusions: The present study suggests that a final common pathway of pancreatic stellate cell activation leads to fibrogenesis in Tropical Calcific Pancreatitis just as in other pancreatic pathologies.

\section{CP-C34 \\ Surgery Increases Long-term Outcomes for Bile Duct Stricture Due to Chronic Pancreatitis}

\section{J. M. Regimbeau, D. Fuks, R. Delcenserie, E. Bartoli}

Department of Digestive Surgery and Gastroenterology, Amiens North Hospital, Amiens, France

Aim: To compare the outcome of endoscopic (ET) and surgical therapy (ST) for benign bile duct stricture (BBS) due to chronic pancreatitis. Methods: From 2003 to 2009, 39 patients were referred in our centre for BBS due to chronic pancreatitis. Sixteen (41\%) patients underwent only transpapillary drainage (with tube exchange every 6 months or in case of obstruction) and 23 underwent surgical biliary drainage (after endoscopic procedures $n=17$ ). Successful therapy was defined as a stent-free condition with no hepatic disorders (bilirubin, alkaline phosphatase). The follow-up was $>6$ months for all patients. Results: Among patients treated by ET, mean number of biliary procedures was $3(1-10)$ per patient (versus 1 for patients with ST) and the mean number of stent was 1.2 per procedure (metallic stents $35 \%$, multiple plastic stents $60 \%$ ). The mean length of stent intubation was 11 months. ET resolved jaundice in all jaundiced patients (10/10) and provided standardization of liver enzymes in $10 / 16(63 \%)$. In ST group, the number of endoscopic procedures prior to surgery was $3(1-10)$. The surgical procedure associated with biliary drainage (choledocho-duodenostomy $n=18$, choledochojejunostomy $n=3$ ) was a Frey procedure in 12 patients, a pancreaticojejunostomy in 2 patients and left pancreatectomy in 2 patients. Success rate of ST was not significantly different from ET $(87 \%$ vs. $63 \%, \mathrm{p}=0.08)$. The success was significantly longer (16.9 vs. 5.8 months, $p=0.01$ ) in ST. The actuarial rate of success at 6-, 12- and $24-$ month was $73 \%, 73 \%, 67 \%$ respectively in the ST group and $75 \%$, $67 \%$ and $0 \%$ respectively in the ET group $(\mathrm{p}=0.04)$. Beyond 3 endoscopic procedures, the success rate at 6 and 18 months was $27 \%$ and $18 \%$ respectively. Conclusion: Surgery increases long-term outcomes for bile duct stricture due to chronic pancreatitis. Beyond 3 endoscopic procedures, the success rate is low.

\section{CP-C35 \\ Pancreatitis in the Setting of the Metabolic Syndrome}

\author{
G. Rajesh, M. Saumya, H. Kumar, V. Balakrishnan \\ Departments of Gastroenterology and Endocrinology, \\ Amrita Institute of Medical Sciences, Cochin, Kerala, India
}

Background: It has been hypothesized that non-alcoholic fatty pancreatic disease (NAFPD) may progress to non-alcoholic steatopancreatitis (NASP) in a manner similar to non-alcoholic steatohepatitis (NASH). There is paucity of literature on the relation of obesity with recurrent- and chronic pancreatitis. Methods: We recorded the clinical details and the outcome of 6 cases of recurrent pancreatitis on follow-up in our Pancreas Clinic who had 3 or more components of the metabolic syndrome. Results: The age of the patients ranged from 8 to 20 years. All the 6 patients had acanthosis nigricans. Body mass index (BMI) had limitations as these patients lost weight following episodes of pancreatitis. Four patients had 2 or more first-degree relatives who were diabetic. Only 1 patient had severe necrotizing pancreatitis. Coexisting liver disease was seen in 3 patients. Elevated serum cholesterol levels and moderate levels of serum triglycerides along with elevated serum amylase levels observed in these patients suggest possibility of a different mechanism from that of hypertriglyceridemia-related pancreatitis. Conclusions: Further characterization of non-alcoholic steatopancreatitis as a clinical entity may help in determining whether active measures in the treatment of metabolic syndrome are helpful in the prevention of repeated attacks of pancreatitis or progression to chronic pancreatitis. 
Miscellaneous

\section{M1 \\ Peculiarities of Pathogenesis of Pancreatogenic Duodenal Ulcers in Patients with Exocrine Insufficiency of Pancreas}

\author{
N.B. Gubergrits, E.L. Balantsova \\ Donetsk Medical National University, Ukraine
}

Introduction: Patients with chronic pancreatitis are often diagnosed duodenal ulcers that can have different pathogenesis. Treatment depends on variant of pathogenesis. Aim: To study peculiarities of pathogenesis of duodenal ulcers in patients with chronic pancreatitis. Methods: 112 patients were examined. Chronic pancreatitis was diagnosed in the presence of typical clinical findings, characteristic changes of indices of pancreatic enzymes of blood and urine, duodenal contents. Fecal elastase test and respiratory test on Helicobacter pylori were carried out. To trial were included patients in whom duodenal ulcers and/or erosion were revealed by endoscopy. 30 healthy persons were examined. Results: According to the results of fecal elastase decrease of exocrine secretion of pancreas was revealed in 63 $(56.2 \%)$ patients, and pancreatic insufficiency was diagnosed in 71 (63.4\%) patients by means of direct (probe) method. Debit-hour of bicarbonates was lowered in $54(48.2 \%)$ patients. The respiratory test was positive in 61 (54.5\%) patients. For reduction of pain $24(21.4 \%)$ patients received nonsteroid antiinflammatory agents more often, than 3 times a week, that also could cause ulcer formation. Conclusion: The most frequent mechanism of formation of duodenal ulcers in chronic pancreatitis is decrease of production of bicarbonates. Practically with the same frequency infection Helicobacter pylori is revealed. The role of nonsteroid antiinflammatory agents in ulcer formation can take place in the fifth part of patients.

\section{M2 \\ Functional State of Pancreas in Patients with Rheumatic Fever}

N.B. Gubergrits, G.M. Lukashevich

Donetsk National Medical University, Ukraine

Background: Visceral manifestations of rheumatic fever especially changes of digestive organs are studied insufficiently. Aim: To study exocrine and endocrine functions of pancreas in patients with rheumatic fever. Methods: 100 patients were examined. Acute rheumatic fever was diagnosed in 22 patients, chronic rheumatic cardiac disease - in 78. Fecal elastase test, direct probe examination of exocrine function of pancreas (aminophylline calcium test) were carried out. Double saccharic load was conducted for estimation of endocrine function of pancreas. Results: Various manifestations of dyspepsia were revealed in one third of cases. $21(21.0 \%)$ patients had mild pancreatic insufficiency according to results of fecal elastase test. By results of probe examination in active phase of disease normal debithour of lipase was found out only in $30(30.0 \%)$ patients, in an inactive phase - in $58(58.0 \%)$ patients. Disorders of endocrine function were diagnosed in 15 patients. All these patients had an active phase of disease with evident articular syndrome. These disorders remained in an inactive phase only in 4 patients. Conclusions: Patients with rheumatic fever have often developed dyspepsia that in some cases can be explained by exocrine insufficiency of pancreas. Disorder of endocrine function of pancreas is possible in active phase of disease.

M3

\section{Functional State of Pancreas in Chronic Abdominal Ischemia}

\author{
N.B. Gubergrits, K.A. Voronin \\ Donetsk National Medical University, Ukraine
}

Background: Blood flow in pancreas disturbs in atherosclerosis of abdominal aorta and its branches that lead to decrease of pancreas function. Aim: To study exocrine secretion of pancreas in patients with chronic abdominal ischemic syndrome (AIS). Methods: 120 patients with AIS owing to atherosclerosis were examined. Fecal elastase test, direct examination of exocrine function of pancreas (aminophylline-calcium test) were carried out. Presence of AIS was confirmed by dopplerography results. Results: Normal indices of elastase test remained only in $9(7.5 \%)$ patients. Severe pancreatic insufficiency was diagnosed in $16(13.3 \%)$ patients, moderate - in 35 $(29.2 \%)$ patients, mild - in $60(50.0 \%)$ patients. According to results of aminophylline-calcium test hyposecretory type of pancreatic secretion was revealed in $81.5 \%$ of cases, low obturative type - in $12,3 \%$ of cases, upper obturative type of exocrine secretion of pancreas - in $6,2 \%$ of cases. Conclusions: Overwhelming part of patients with AIS has exocrine insufficiency of pancreas demanding replacement therapy by enzyme preparations.

\section{M4}

Diabetes Induced Oxidative Stress and $\beta$-cell Dysfunction: Role of Tinospora Cordifolia

\author{
M.K. Sangeetha, A. Hannah, R. Vasanthi
}

Herbal and Indian Medicine Research Laboratory, Sri Ramachandra University, Chennai, Tamilnadu; Department of Biotechnology, School of Life sciences, Pondicherry University, Puducherry, Tamilnadu, India

Background: Oxidative stress is one of the key factors involved in the development and progression of type 2 diabetes. Aim: To study the antidiabetic potential of an traditional plant Tinospora cordifolia on oxidative stress, insulin resistance and â cell dysfunction in in-vivo and in-vitro experimental conditions. Methods: (1) In-vivo experimental animal study: Diabetes was induced in rats with High fat diet coupled with low dose of streptozotocin as this model closely mimics pathogenesis of humans. Circulating glucose was estimated 
by glucose oxidase kit and insulin by Enzyme Linked Immuno Sorbent Assay (ELISA). Histopathological changes and oxidative stress markers in pancreas were analyzed. (2) In-vitro cell line study: $\beta$-TC6 cell line is derived from transgenic mice, were used to study the insulin secreting property and antioxidant efficiency of Tinospora cordifolia. Results: Treatment with Tinospora cordifolia (200mg/ $\mathrm{kg}$ ) prevented diabetes induced oxidative stress in pancreas and insulin resistance $(p<0.001)$. Histopathological analysis showed regeneration of cells when compared to atrophied diabetic control cells. In In-vitro, Tinospora cordifolia showed antioxidant property but failed to produce insulin secreting property. Conclusion: Tinospora cordifolia, an antidiabetic herb used from time immemorial showed its antioxidant property in both in-vivo and in-vitro model, whereas the insulin resistance and insulin release was preserved only in in-vivo.

\section{M5}

Management and Outcomes of Patients with Insulinoma at a Tertiary Care Hospital in India: A Retrospective Analysis

\author{
G. Choubal, S. Pal, P. Sahni, T.K. Chattopadhyay, \\ S. Gamanagatti, N. Tandon, R. Sharma, P.K. Garg, \\ V.P. Jyotsna, A.C. Ammini \\ Departments of Endocrinology, Radiology, \\ Gastroenterology and GI Surgery, All India Institute of \\ Medical Sciences, New Delhi, India
}

Introduction: Insulinomas are the commonest islet cell tumors of the pancreas. Their localisation can be difficult but surgery is curative as most of these tumours are benign. Methods: We studied all patients operated for hyperinsulinemic hyperglycemia (1992-2010). Their demographic parameters, symptoms, biochemical profile, imaging studies, intra-operative ultrasound (IOUS) and type of surgery, histopathology and postoperative outcome were analyzed. Since 2008, endoscopic ultrasound (EUS), DOTATOC-PET-CT and intra arterial calcium stimulation tests (IACS) have also been used for preoperative localisation. Intraoperatively, inspection, palpation and IOUS of the pancreas were done. Enucleation, distal pancreatectomy (DP) or pancreatoduodenectomy (PD) was done based on the location and size of the tumour. Results: A total of 51 (28 males; mean age: $37 \mathrm{y})$ cases were studied. Mean duration of symptoms was 4.6 years. Spontaneous biochemical diagnosis was made in 56\% cases, ' 72 -hour fast' was positive in remaining $43 \%$ cases. Dual phase CECT/MRI could localize in 50-55\% cases while intraoperative localisation was possible in 91\%. Pick-up rates of EUS and IACS were $85.7 \%(6 / 7)$ and $75 \%(3 / 4)$ respectively. Insulinomas were located in the pancreatic head, body and tail in 15 (29.4\%), $13(25.4 \%)$ and $23(45 \%)$ respectively. Two patients had multiple tumours, 1 had nesidioblastosis. Three had malignant insulinomas (1 had MEN syndrome). Enucleation, DP and PD were done in 25 (49\%), 24 (47\%) and 2 (4\%) cases respectively. Two patients (multiple insulinomas -1, nesidioblastosis-1) had recurrence of hypoglycaemia, of which 1 was successfully reoperated. Four patients developed transient pancreatic fistulae and 2 died of sepsis and delayed haemorrhage. Conclusion: Accurate perioperative localization of insulinomas was achieved in $91 \%$ and surgery was successful in $94 \%$ cases.
Selective use of EUS and IACS has the potential to improve localization.

\section{M6 \\ Three Year Followup of Non Functioning Islet Cell Tumor of Pancreas in an Adolescent Girl}

P. Sriram, N. Venu, N. Reddy, Gopinath

Department of Surgical Gastroenterology, Sri

Venkateswara Institute of Medical Sciences, Tirupati, India

Background: Tumors of pancreas in adolescent age is rare. The Case: We report a case in a 14 year old girl of arising from uncinate process who sucessfully underwent Whipple's pancreatico duodenectomy and is recurrence free 3 years post resection. Patient presented with vague abdominal pain of 2 months duration. She was well preserved and anicteric. Examination revealed a vague nontender mass in epigastrium. Liver biochemistry was normal. USG abdomen revealed mixed echogenic mass in relation to head of pancreas. CECT abdomen revealed mixed dense mass at uncinate process of pancreas with loss of fat planes over superior mesentric vessels. There was no intrahepatic biliary dilatation. She underwent Whipple's pancreaticoduodenectomy in February 2007. The tumor was pushing the superior mesentric vein; there was no infiltration. Cut section revealed an encapsulated tumor with solid and necrotic elements. Histopathology revealed pancreatic neoplasm with no mitotic figures. Immunohistochemistry revealed neoplastic cells positive for synaptophysin, and chromagranin and negative for CEA. She is on periodic follow-ups. At final followup of 3 years, liver was normal and there was no local recurrence on CECT abdomen. Conclusion: Nonfunctioning Islet cell tumor of Pancreas (NFICT) rarely present in younger age group. Even if imaging modalities shows vessel infiltration, aggressive surgical resection should be considered as the treatment of choice.

\section{M7 \\ Endoscopic Management of Pancreatic Injury Due to Abdominal Trauma}

\author{
D.K. Bhasin, S.S. Rana, R. Gupta, M. Kang, B. Nagi, \\ B.R. Thapa, K. Singh \\ Departments of Gastroenterology, Surgery and \\ Radiodiagnosis, Post Graduate Institute of Medical \\ Education and Research (PGIMER), Chandigarh, India
}

Aims: Pancreatic injury is uncommon consequence of abdominal trauma and is usually managed surgically. We report our experience of endoscopic management of pancreatic trauma. Methods: Over last 6 years, 8 patients with pancreatic trauma were treated with endoscopic therapy. The patients with pseudocyst and gastroduodenal bulge were treated with transmural drainage and without bulge were treated with transpapillary drainage. Results: Six 
patients $(5 \mathrm{M})$ were treated in chronic phase ( $>4$ weeks after trauma) and 2 patients $(2 \mathrm{M})$ were treated in acute phase. Both patients presenting in acute phase underwent transpapillary drainage after undergoing percutaneous drainage for symptomatic acute fluid collections. One patient had partial disruption at tail end and was successfully treated with bridging pancreatic stent. Patient with complete disruption had non resolution after placement of non bridging pancreatic stent and required surgery. Six patients presenting in chronic phase had symptomatic pseudocyst. Two patients were successfully treated with cystogastrostomy and there has been no recurrence over a follow up of 16 and 12 months. Four patients underwent transpapillary drainage and 3 patients had partial disruption and 1 had complete disruption. Bridging nasopancreatic drain (NPD) was placed in 1 patient and stent in 2. All 3 patients had resolution of pseudocyst within 6 weeks and there has been no recurrence. Patient with complete disruption and non-bridging stent required surgery because of non resolution. Conclusion: Pancreatic injury due to trauma can be effectively treated endoscopically and patients with complete disruption usually have unfavorable outcome after transpapillary drainage.

\section{M8}

\section{Management Outcome in Pancreatic Trauma}

\section{M.A. Johnson}

Department of Surgery, Kanyakumari Medical College, Kanyakumari, India

Background: Following pancreatic trauma, pseudo cysts develop in $40 \%$ to $100 \%$, but abscesses are not uncommon, and recurrent episodes of pancreatitis may occur remote to the time of injury. Non operative management (NOM) of pancreatic transection or ductal disruption can, however, be costly. Aim: To evaluate the safety, efficacy and clinical outcome of different surgical procedures performed in managing pancreatic trauma with an intent to setup management guidelines/treatment protocol. Methods: A prospective analysis of a cohort of 16 pancreatic injury patients, blunt $n=16$, motor vehicle accident $n=11$, bicycle handle bar injury $n=2$, fall from a height $\mathrm{n}=3$ during the period from 2003 to 2009. Among 16 patients, there were 11 men, 2 women, 3 girl children. The incidence of AAST grade I to $\mathrm{V}$ pancreatic injuries were $40.0 \%$, $18 \%, 27 \%, 0 \%$, and $15 \%$ respectively. Time from injury to hospital presentation was 6-8hours. Time from hospital presentation to diagnosis of injury was $10-12$ hours. Time from injury to operation was 24-28 hours. Surgery was required in 10 patients, which included ligation of bleeding vessel and peri-pancreatic drainage in three, central pancreatectomy in six and pancreaticoduodenectomy in one patient respectively. Results: Overall morbidity rate was $54.5 \%$ which included intra-abdominal abscess in two, pseudocyst in two, pancreatitis in one and pancreatic fistula in one patient respectively with no mortality. Conclusion: A high index of clinical suspicion and repeated computed tomography scans should lead to earlier diagnosis in pancreatic trauma. The complication rate for major blunt pancreatic injury was high; especially when treatment was delayed for more than 24 hours. Central pancreatectomy with pancreaticogastrostomy is a safe pancreas preserving conservative surgical strategy worthy of consideration as an alternative to distal pancreatectomy in the setting of traumatic complete transection of pancreatic neck.

\section{M9 \\ Current Management Protocol in Peri- pancreatic Pseudoaneurysms}

\section{M.A. Johnson, S. Chidambaram \\ Department of General Surgery, Kanyakumari Government Medical College, Kanyakumari, India}

Background: Haemosuccus pancreaticus and haemobilia are rare causes of obscure G.I.bleed that poses considerable diagnostic and therapeutic challenges. Endovascular treatment has recently been advocated as the preferred therapy. Aim: (1) To highlight the need for increased awareness of such a rare clinical entity (2) To evaluate the safety, efficacy and outcome of non-surgical/surgical treatment options for pseudoaneurysmal bleed with an intent to setup management guidelines. Methods: Prospective analysis of 16 patients (12 men, 4 women) from 1998 to 2008.11patients had bleeding PAs complicating pancreatitis (chronic in 10 and acute in one), post-traumatic in two patients following blunt liver injury, and post-operative in 3 patients, following laparoscopic cholecystectomy in two and whipple's pancreatoduodenectomy in one. Results: Angiography performed in $11(68.75 \%)$ with Transcatheter angiographic embolization (TAE) attempted in $9(81.8 \%)$ and achieving temporary hemostasis in $6(66.6 \%)$. Technical failure occurred in $3(33.3 \%)$, secondary intraabdominal sepsis in one $(11.1 \%), 14$ days after embolization and rebleeding in one patient, 2 days after embolization respectively, and were successfully treated by elective operation. 12 patients underwent operative therapy. Morbidity and rebleeding in the angiographic intervention group were $11.1 \%$ each respectively. Surgical morbidity was $41.6 \%$ with no rebleeding. No mortality in either group. No recurrence of pseudoaneurysm or bleeding after a mean (range) follow-up of 26.3 (45-59) months. Conclusion: TAE should be considered as the first line treatment of choice in all patients presenting with pseudoaneurysmal bleed. Surgery should be reserved only for (i) actively bleeding lesions unsuitable for angioembolization with significant haemodynamic instability (ii) non-availability or failure of embolization (iii) secondary complications such as extrinsic compression or sepsis.

\section{M10 \\ Predictors of Mortality and Outcome of Non Operative Management, in Grade III and IV Blunt Pancreatic Trauma}

\author{
K. Ravikanth, B. Anand, V. Gandhi, S. Rasesh, \\ K. Suman Bhushan, N. Bheerappa, R. A. Sastry \\ Nizam's Institute of Medical Sciences, Hyderabad, India
}

Background: Although surgery is the standard treatment for grade III and IV pancreatic trauma, there are reports of success with non operative management (NOM) too. Objective: To analyze the role of NOM and analyze the predictors of mortality and outcome of NOM after grade III and IV pancreatic trauma. Methods: 60 patients with blunt pancreatic trauma presenting to our hospital over past 13 years were studied. Patients with grade I, II and V pancreatic injury and those associated with major injuries to other organ systems were 
excluded. Of the 44 patients that were included in the study, nine patients were operated early $(<48 \mathrm{hr})$ and formed the operative management (OM) group. Rest of the 35 patients was classified into NOM group. Data regarding demographics, management strategy (NOM vs. OM), complications, and outcomes were collected from a prospectively maintained database. Univariate and multivariate analysis were performed using SPSS 18. Results: Twenty of the 35 patients in the NOM group required surgery later and were considered failures. All the five deaths occurred in this group. Pancreas specific complications were higher in NOM group ( $88 \%$ vs $33 \%$ p0.0001). The significant predictors of failure of NOM on univariate analysis were grade of injury (p:0.012), pancreatic contusion on CT (p:0.013), presence of ileus (p:0.001), associated pancreatitis (p:0.0001), presence of necrosis (p:0.0001), overall pancreatic complications (p:0.014). Pseudocyst (p:0.005) was the only factor predicting success of NOM. On binary logistic regression only grade of injury and necrosis were significant factors. The significant predictors of mortality on univariate analysis were female sex (p:0.005), pancreatic contusion on CT (p:0.036), presence of ileus (p:0.004), associated pancreatitis (p:0.001), pancreatic necrosis(p:0.009) and failure of NOM (p0.034). On binary logistic regression only associated necrosis (p:0.02) was found to be significant factor. Conclusions: Grade III and IV injuries, have high mortality when not operated. They should be operated when associated with contusion in addition to ductal transaction. Presence of necrosis and grade of injury are the significant factors influencing outcome of NOM in grade III and IV injuries, while associated necrosis is the only significant factor influencing mortality.

\section{M11 \\ Spontaneous Pancreaticocutaneous Fistula Following Successful Endoscopic Pancreatic Pseudocyst Drainage}

\author{
N. Nadkarni, M. Jayant, S. D'Cruz, R. Kaur, A. Sachdev \\ Department of Medicine, Giansagar Medical College, \\ Punjab; Departments of Surgery, Medicine and \\ Radiodiagnosis, Government Medical College, \\ Chandigarh, India
}

A 40 year old male with a past history of pancreatitis following a blunt abdominal trauma, presented with pain and lump in the upper abdomen. On examination he had a palpable lump in the epigastrium. Ultrasound abdomen revealed a pseudocyst of the pancreas of size 15 $\mathrm{cm}$ [maximum diameter]. Contrast enhanced computed tomography of the abdomen also revealed a similar sized pseudocyst abutting the stomach with no evidence of segmental portal hypertension or portosystemic collaterals. The patient was subjected to endoscopic cystogastrostomy, after which he had marked improvement in his symptoms. The patient got readmitted one week later with pain in the epigastrium and something coming out of the abdominal wall. On examination a pigtail stent was projecting out partially from the abdominal wall in the region of the scar of previous laprotomy. USG revealed that the residual cyst size was $3 \mathrm{~cm}$ with one end of the stent in the cyst cavity. The findings were suggestive of pancreatico-cystocutaneous fistula. The displaced stent was removed and patient was subjected to ERCP. Pancreatogram revealed pancreatic duct disrup- tion across which stent was placed. Presently after 2 months of ERCP patient is asymptomatic.

\section{M12 \\ Magnetic Resonance \\ Cholangiopancreatography Findings in 35 Patients with Chronic Asymptomatic Hyperenzimemia

\author{
G. Leonardi, M. Del Chiaro, N. de Bortoli, U. Boggi, \\ S. Marchi \\ Gastroenterology Unit, Pisa University Hospital, Italy; \\ Departments of General and Transplant Surgery, Pisa \\ University Hospital, Italy
}

Background: Persistent increases in serum concentrations of amylase and/or lipase without clinical evidence of pancreatic disease are a relatively common finding in clinical practice. Aim: to analize the spectrum of MRCP findings in patients with asymptomatic hyperenzimemia. Methods: Over an 18-month period, 35 patients having chronic hyperenzimemia were scheduled to undergo MRCP. 10 patients had pancreatic hyperamylasemia, 4 only hyperlipasemia and 21 an increase of both enzymes. The increase of pancreatic enzymes was found occasionally and was confirmed by repeated laboratory tests. None of the subjects had any symptoms or signs of pancreatic or other diseases; serum cholesterol and triglyceride levels were normal. All patients were no drinker or at least $<40 \mathrm{gr} /$ die of alcohol. Results: Only 8 out of 35 patients $(23 \%)$ had a normal pancreatic ductal three at MRCP. In the remaining 27 patients (77\%), 8 pts $(29.5 \%)$ showed pancreatic cysts, $10(37 \%)$ pancreatic changes compatible with IPMN, $1(3.7 \%)$ a dilated Wirsung with Kinking, 1 (3.7\%) IPMN and chronic pancreatitis, 1 (3.7\%) IPMN and cancer, 1 $(3.7 \%)$ a neuroendocrine tumor, $2(7.5 \%)$ a chronic pancreatitis, 1 (3.7\%) an autoimmune pancreatitis and 2 (7.5\%) a 'Pancreas Divisum'. Conclusion: MRCP is the most effective and non invasive imaging technique in patients with asymptomatic hyperenzimemia because it gives very detailed morphological information about the pancreratico-biliary ductal system. In $77 \%$ of cases we found clinical relevant diseases (IPMN, cancer, pancreatic cysts). A long term follow-up is necessary to set up the clinical meaning of some of the MRCP alterations.

\section{M13 \\ Conservative Treatment as an Option for Complete Pancreatic Ductal Transaction}

\author{
D. Balsarkar, R. Patil, S. Baranwal \\ B.Y.L. Nair Hospital and T.N. Medical College, Mumbai, \\ Maharashtra, India
}

Background: Pancreatic trauma following blunt injury is uncommon. Surgery and endoscopy has been the mainstay of treatment for complete pancreatic duct disruption. Early diagnosis and 
prompt treatment is the key to successful outcome. We present a successful outcome in a conservatively managed complete pancreatic ductal disruption in the neck region associated with Grade 3 left lobe liver laceration. Clinical history: A 25 years old male presented following fall from height under the influence of alcohol with blunt abdominal trauma. On presentation patients pulse was 110 per minute and systolic blood pressure of $90 \mathrm{~mm} \mathrm{Hg}$. CT scan after initial resuscitation showed a grade 3 left lobe liver laceration with complete pancreatic duct disruption in the neck region along with mild hemoperitoneum. Since patient was hemodynamically stable and hemoglobin values remained constant at $9 \mathrm{gm} \%$, a decision to treat the patient conservatively was made. During hospital stay patient developed pancreatic ascites, which was treated with USG guided pigtail catheter drainage. Nutrition was taken care by naso-jejunal tube for providing enteral feeds. Twice ERCP was attempted which was unsuccessful. At ERCP a 7 Fr stent, could be inserted only till the neck region into the collection. Patient had a hospital stay of 3 months, during which time he was serially monitored with CT scan and MRCP evaluation. At discharge patient was fine and eating orally with no abdominal pain. USG at discharge showed a small 2.8 by $2 \mathrm{~cm}$ collection in the body region of the pancreas which was seen to communicate with the PD. Rest of the pancreas was normal. Patient is asymptomatic at one year since conservative treatment. Conclusion: Conservative treatment can be used in select patients with complete pancreatic ductal transaction after having ruled out other injuries requiring intervention. ERCP with PD stenting should be attempted if possible. Aggressive management of collections with imaging guided pigtail catheter drainage may be required. Nutrition can be taken care by insertion of Nasojejunal tube. Serial monitoring using USG, CT scan and MRCP is needed to be performed during the course of conservative management.

\section{M14 \\ Pancreatic Problems in Women: A Series of 14 Cases}

N. Srinivas, P. Murali Krishna, L. Girinadh, A. Aditya, Md. Akbar

Department of Gastroenterology, Andhra Medical College, Visakhapatnam, Andhra Pradesh, India

Background: Studies conducted to analyse epidemiology of pancreatic diseases in India shows idiopathic as most frequent etiology and alcoholic as next most common while there is a paucity of such data for women group of India in whom alcoholic consumption is very rare. Aim: To analyze a tertiary care experience with various pancreatic problems in women and clarify the frequency of various pancreatic diseases in the same patient population as well as the role of surgical or conservative management in their treatment. Methods: All female patients with clinical suspicion of disease pertaining to the pancreas, substantiated by laboratory investigations or imaging modalities are included in our study. Results: Of the total 14 patients in age group of 11-55 years, with various pancreatic problems. 7 cases are of acute pancreatitis and 7 presented with acute exacerbation of chronic pancreatitis. 2 cases are of Fibrocalculous pancreatic diabetes, 2 cases have choledochal cyst while 1 patient has hypertriglyceridemia. 1 patient had possibly drug induced (mesala- mine) pancreatitis and 1 case was due to pancreatic duct injury during surgery and 1 patient with carcinoma pancreas presented with acute pancreatitis and the etiology in 6 cases is unknown. None has gall stones. Most patients (12) improved with conservative treatment and 1 needed surgery (pancreatojejunostomy) while another other patient is under chemotherapy for carcinoma pancreas. Conclusion: Pancreatic disorders are not so infrequent in women and have a diverse etiology. Hence a high index of suspicion aided by the newer modalities of investigation greatly helps in the appropriate and timely management.

\section{M15 \\ Collagen-I Increases the Exression of Transgelin and Lumican (Regulators of Cell Migration) by Activated Pancreatic Stellate Cells}

\author{
L. Yang, P. Phillips, Z. Xu, J. Youkhana, A. Vonlaufen, \\ R. Pirola, J. Wilson, M. Apte \\ Pancreatic Research Group, University of New South \\ Wales, Sydney, Australia
}

Background: Activated pancreatic stellate cells (PSCs) play a central role in pancreatic fibrosis, a feature of chronic pancreatitis and pancreatic cancer. The fibrous matrix produced by PSCs can, in turn, regulate PSC function. Using microarrays, we have previously demonstrated that 146 genes were dysregulated (fold change $>2, p<0.001$, $\mathrm{q}<0.25, \mathrm{n}>3$ ) in PSCs cultured on collagen-I (mimics fibrotic pancreas) versus Matrigel ${ }^{\mathrm{TM}}$ (mimics normal pancreatic basement membrane). Interestingly, genes for transgelin and lumican (which code for proteins known to regulate cell migration) were upregulated in PSCs cultured on collagen-I compared to Matrigel. Aim: To assess transgelin and lumican mRNA and protein expression in: i) PSCs cultured on matrigel versus collagen-I; ii) quiescent PSCs vs PSCs cultured on plastic. Methods: Transgelin and lumican expression was assessed by real-time PCR and western blotting in quiescent rat PSCs (24 hours after isolation or grown on Matrigel ${ }^{\mathrm{TM}}$ and activated PSCs (cultured on collagen-I for $72 \mathrm{~h}$ or on plastic); $\mathrm{n}=5$ preparations). Results: Transgelin and lumican mRNA levels were upregulated by i) 45.5 and 24.7 fold $\left({ }^{*} p<0.05\right)$ respectively in PSCs cultured on collagen-I vs Matrigel ${ }^{\mathrm{TM}}$. and ii) 20 and 4.2 fold $\left({ }^{*} \mathrm{p}<0.05\right)$ respectively in plastic-activated PSCs vs quiescent cells. Transgelin protein expresion was also increased ( $\%$ of control [mean $\pm \mathrm{SE}$ ]: $\left.295.7 \pm 28.92,{ }^{*} \mathrm{p}<0.05\right)$ in activated PSCs compared to quiescent PSCs. Conclusions: Transgelin and lumican are significantly increased in PSCs cultured on fibrous ECM and associated with PSC activation. Implication: Characterisation of genes that may play a role in PSC transformation may allow the identification of specific therapeutic targets for the treatment of fibrosis. 


\section{M16 \\ Comparison of Diagnostic Yields of Endoscopic Ultrasound (EUS) and Ultrasound (US)/ Computed Tomography (CT) Guided Fine Needle Aspiration (FNA) of Pancreatic Masses}

\author{
R. Srinivasan, S.S. Rana, D.K. Bhasin, N. Kalra, K. Singh \\ Departments of Cytology, Gastroenterology and \\ Radiodiagnosis, Postgraduate Institute of Medical \\ Education and Research (PGIMER), Chandigarh, India
}

Background: EUS FNA is an important diagnostic tool for evaluation of pancreatic masses. There is limited experience on its diagnostic yield with that of US/CT guided FNA. Aim: To compare the diagnostic yield of EUS-FNA with that of US/CT guided FNA for diagnosis of pancreatic mass lesions. Methods: Over last 18 months, 40 patients with pancreatic mass (solid/cystic) lesions presumed to be resectable on CT abdomen were subjected to EUS FNA. During the same period 114 patients underwent US/CT guided FNA. The final diagnosis was obtained either by surgery or endoscopic retrograde Cholangiopancreatography (ERCP) or after clinical follow up. Results: The final diagnosis in patients with pancreatic mass lesion who underwent EUS FNA was: adenocarcinoma (14), Pseudocyst (10), inflammatory mass (4), neuroendocrine tumor (4), pancreatic tuberculosis (3), pancreatic metastasis from renal cell carcinoma (1) simple cysts (1), mucinous cystadenoma (1), serous cystic tumor (1) and intraductal papillary mucinous tumor (1). EUS guided FNA yielded the correct diagnosis on first attempt in 37/40 (92.5\%) patients. Three patients required repeat FNA for achieving the correct diagnosis. The final diagnosis in 114 patients with pancreatic mass lesion who underwent US/CT guided FNA was: adenocarcinoma (43), Pseudocyst (12), autoimmune pancreatitis (6), inflammatory mass (5), solid pseudopapillary tumor (4), neuroendocrine tumor (3), pancreatic tuberculosis (3), non Hodgkin lymphoma (1), and serous cystic tumor (1). A definite diagnosis could be made and a descriptive report was given in 36/114 (31.6\%) patients. US/CT guided FNA yielded the correct diagnosis on first attempt in $78 / 114(68.4 \%$ ) patients. No major complications were noted either of EUS or CT/ EUS guided FNA. Conclusion: EUS FNA gives higher yield than $\mathrm{CT} / \mathrm{US}$ guided FNA for establishing diagnosis in pancreatic mass lesions.

\section{M17 \\ Traumatic Pancreatitis Masquerading Acute Obstruction in Pediatric Age; Experience of Two Cases in Rural Area}

\author{
S. Dwivedi, S.S. Trehan \\ Surgical Discipline, M.M. Institute of Medical Sciences \\ and Research, M.M. University. Mullana, Ambala, India
}

Introduction: Despite blunt trauma abdomen being the known cause of pediatric pancreatitis, the diagnosis and management is often difficult. Its presentation as acute upper GI obstruction warranting surgical exploration is a matter of discussion. Case history: Two children age 6 years and 8 years respectively sustained blunt pancreatic trauma (BPT) with handle of bicycles within one week and admitted with classic presentation of acute intestinal obstruction in emergency. Serial radiographic imaging, Serial serum amylase and lipase and ultrasound with emergency CT scan were predicting hemorrhagic pancreatitis but did not predict injury severity or need for further intervention and dilemma was persisting as both were deteriorating on conservative line of therapy. Finally in view of persisting upper GI symptomatology, exploratory laparotomy was planned. Final intra-operative findings and diagnosis warranted the discussion about early surgical exploration and its feasibility? Both these patients survived and are doing well with almost one year follow up. Conclusions: A combination of serial radiographic and USG, laboratory and ERCP when clubbed with emergency CT scan will improve the diagnosis and management of BPT with suspected traumatic pancreatitis. Normally ductal injuries require surgical intervention, and fluid collections may require drainage otherwise most BPT can be managed non-operatively with minimal morbidity and mortality. Some times BPT may present with atypical finding and masquerading with GI obstruction non responsive to conservative protocol. Should it be wise to advise surgical exploration in such BPT cases especially in pediatric age group?

\section{M18 \\ Development of a Technique to Isolate $T$ Lymphocytes from Pancreatic Tissue}

\author{
J. Jupp, C. Johnson, D. Fine, S. Gadola \\ University of Southampton, UK
}

Background and Aims: T lymphocytes infiltrate pancreatic tissue in chronic pancreatitis (CP). Techniques to isolate $\mathrm{T}$ lymphocytes from pancreatic tissue are therefore central to allow investigation of the role of the immune system in CP. Methods: Five patients with CP (all male, aged 35-62, median age 46) and two patients with pancreatic cystic disease that donated normal pancreatic tissue (one male, one female, ages 68 and 75) were evaluated. Tissue was finely diced and then enzymatically digested using Collagenase II before passing through a $100 \mu \mathrm{m}$ nylon mesh. Cells were subsequently washed in a buffer containing ascorbic acid, $\mathrm{N}$-acetylcysteine, glucose and FBS, in addition to EDTA and Pefabloc, which halt further enzymatic digestion. Lymphocytes were isolated using density gradient centrifugation and analysed by flow cytometry with a FACSAria using fluorochrome conjugated antibodies (anti-CD3/CD4/CD8) and a live/ dead stain. Results: The maximum yield of live CD3+ T lymphocytes from CP tissue was 150,000 per gram of tissue resected. Tissue from patients with $\mathrm{CP}$ tended to yield higher numbers of $\mathrm{T}$ lymphocytes compared to controls. Higher yields of $\mathrm{T}$ lymphocytes were associated with loss of CD4 from the cell surface. T lymphocytes in $\mathrm{CP}$ tissue digests consisted of approximately equal proportions of CD8+ (46.7 +/- 19.5\%) and CD4+/DN (43.0 +/- 19.8\%) cells. Conclusion: The described technique reproducibly allows $\mathrm{T}$ lymphocytes to be isolated from pancreatic tissue, permitting further evaluation. A fine balance exists between tissue digestion and loss of CD4 from the surface of T lymphocytes. These preliminary data sug- 
gest $\mathrm{T}$ lymphocyte infiltration into $\mathrm{CP}$ tissue consists of equal numbers of CD4+ and CD8+ lymphocytes.

\section{M19 \\ Effects of Intravenous Administration of Pentoxifylline in Pancreatic Ischemic/ Reperfusion Injury}

\author{
E.R. Campion, J. Jukemura, A.M.M. Coelho, \\ S.N. Sampietre, N.T. Molan, M.C.C. Machado, \\ L.A.C. D'Albuquerque \\ Departments of Gastroenterology and Biochemistry, \\ University of Sao Paulo, Brazil
}

Background: Therapeutics strategies to reduce pancreatic ischemic/reperfusion injury (IRI) might improve the outcome of human pancreatic-kidney transplantation. Pentoxifylline beside its hemorrheologic effects has an anti-inflammatory effect by inhibiting NF-kappaB activation. We have previously demonstrated that pentoxifylline had anti-inflammatory response in acute pancreatitis and liver ischemia/reperfusion models. We hypothesized that pentoxifylline could reduce pancreatic, renal lesions and the systemic inflammatory response in pancreatic I/R injury. Aim: The aim of this study was to evaluate the effect of pentoxifylline administration in a rat modelofpancreaticischemia/reperfusioninjury.Methods: Pancreatic ischemic/reperfusion was performed in Wistar rats during one hour by clamping the splenic vessels. The vascular clamp was removed and pancreatic revascularization was achieved followed by $4 \mathrm{~h}$ and $24 \mathrm{~h}$ of reperfusion. The animals were submitted to ischemic/reperfusion and divided into 2 groups: Group $\mathrm{C}(\mathrm{n}=20)$ : control, rats received saline solution IV, 45 minutes after ischemia, and Group P $(n=20)$ : rats received pentoxifylline $(25 \mathrm{mg} / \mathrm{Kg}) \mathrm{IV}, 45$ minutes after ischemia. Four and twenty four hours after reperfusion blood were collected for determinations of amylase, creatinine, TNF- $\alpha$, IL-6, and IL-10. Pancreatic malondialdehyde (MDA) content was also performed. After 48 hours of reperfusion pulmonary tissues were assembled for myeloperoxidade (MPO) analyses. Results: A significant reduction in serum TNF- $\alpha$, IL-6, IL-10, and creatinine levels was observed in pentoxifylline group compared to control group $(p<0.05)$. No differences in pancreatic MDA content and in serum amylase levels were observed between two groups. Twenty-four hours after ischemia did not observe any significant difference in the results of lung myeloperoxidase activity (MPO). Conclusion: Pentoxifylline administration reduced the systemic inflammatory response and renal dysfunction in pancreatic $\mathrm{I} / \mathrm{R}$ injury and could be a useful tool in pancreas-kidney transplantation.

\section{M20 \\ Pseudoaneurysm in Pancreatitis: Efficacy of Angioembolisation}

\author{
T.U. Shabeerali, R.S. Sindhu, M.L. Arunkumar, B. Natesh, \\ R. Rajan, A.K. Gupta
}

Departments of Surgical Gastroenterology, Interventional Radiology, Medical College and Sree Chitra Tirunal Institute of Medical Sciences and Technology, Trivandrum, Kerala, India

Background: Literature on clinical profile, management and outcome of pseudo aneurysms (PSA) associated with pancreatitis are limited. Aim: To study clinical presentations and efficacy of angioembolisation (AE) in PSA associated with pancreatitis. Methods: Prospective study of PSA in pancreatitis diagnosed by Contrast Enhanced Computerised Tomography (CECT) or Digital subtraction angiography (DSA), admitted between January 2005 and October 2010, excluding the post-operative PSA. They were managed by observation, Angio-Embolisation (AE) or surgery and followed up for the recurrence of symptoms, morbidities and mortality. Results: 12 PSA associated with pancreatitis patients were admitted during the study period (Male-female: 11:1, mean age: 42 years). Etiologies were chronic calcific pancreatitis (CCP) (7), severe acute pancreatitis (1), recurrent pancreatitis (3) and post traumatic (1). Presentations were upper gastrointestinal bleed (9), bleeding per rectum (1), pain (1) and incidental (1). CECT showed PSA in all; splenic artery (SA) (8), cystic artery (1) or pancreatico-duodenal arcade (4). DSA done in 9, 7 were positive for PSA and AE done in 6(failed in 1 due to torturous SA). 4 had Distal pancreatectomy with Splenectomy (DPS) (failed AE (1), splenic vein thrombosis with fundic varices (1), Symptomatic Pseudo cyst (SP) (1) and patient preference (1)). The DSA negative were observed. With a median follow up of 6 months, there were no mortality or re bleed in either group. One in AE group underwent surgery (Frey's) for CCP with SPc after 4months. One in DPS group had pancreatic fistula. Conclusion: AE appears to be effective in most cases of PSA but some need individualised management for good outcome. 


\section{M21 \\ Single Centre Experience of 144 Children with Pancreatitis}

\author{
M. Bharadwaj, Md. A.G. Khan, N. Mohan \\ Departments of Pediatric Gastroenterology, Hepatology \\ and Liver Transplantation, Medanta The Medicity Hospital, \\ Gurgaon, Haryana, India
}

Aims: To determine the etiology and clinical spectrum of pancreatitis in children presenting to a tertiary health care centre in North India. Methods: Retrospective analysis was done of prospectively collected data of children ( $<16 \mathrm{yrs})$ diagnosed with pancreatitis between Jan 1999 to June 2010. Results: There were 144 children, 84 males. Mean age of presentation was $9.7 \mathrm{yrs}$ (1 to $16 \mathrm{yrs}$ ). There were $62(43 \%)$ acute pancreatitis (AP), 54 (37.5\%) chronic pancreatitis (CP) and $28(19.5 \%)$ recurrent pancreatitis (RP) cases. Etiology of AP included idiopathic 24 (38.7\%), traumatic 16 (25.8\%), infection 8 $(12.9 \%)$, biliary $6(9.6 \%)$, anatomical defects $5(8 \%)$ and drug induced $3(4.9 \%)$. Necrosis was seen in $20(32.25 \%)$, infection $9(14.5 \%)$, shock $4(6.45 \%)$, vascular thrombosis $2(3.2 \%)$, seizures and acute renal failure in one case (1.5\%) each. 10/24 cases of pseudopancreatic cyst (PPC) underwent cystogastrostomy (CG): 9 endoscopic, 1 surgical. There was no mortality. Out of 54 patients of CP, $30(55.55 \%)$ were non calcific. Complications included stricture of PD 12 $(22.22 \%)$, PPC $7(13 \%)$, gland atrophy $4(7.5 \%)$, exocrine pancreatic insufficiency $2(3.7 \%)$, gastroduodenal artery aneurysm and diabetes mellitus (DM) in 1 each. The causes of RP included anatomical defects 4 , hereditary pancreatitis 2 , hypercalcemia 1 and rest idiopathic. Complications in RP were PPC in 4 and shock in 3. Conclusions: Acute idiopathic pancreatitis was the commonest presentation. There was no gender discrepancy. In AP, PPC was seen in one third; more than one third underwent CG. Despite necrosis in few there was no mortality. Non calcific CP was more than calcific $\mathrm{CP}$ in North Indian children. PD Stricture seen in one fifth and PPC in one tenth of CP. DM and steatorrhoea were uncommon.

\section{M22 \\ Post-ERCP Pancreatitis - A Retrospective Study from a Tertiary Care Center}

\author{
R. Maiwall, B.S. Ramakrishna, A. Chacko, S.D. Chowdhury, \\ M. Sahu, P. Alagammai \\ Department of Gastrointestinal Sciences, Christian \\ Medical College Vellore, Tamil Nadu, India
}

Background: There is a paucity of data for post-ERCP pancreatitis from India. Methods: This was a retrospective study done at a tertiary care center in South India. Patients diagnosed with post ERCP pancreatitis requiring hospitalization for more than 48 hours were included in the study. The study was conducted between January 2005 and December 2009. Results: A total of 2131 patients underwent ERCP at our hospital. Of them 20 patients required hospitalization with acute pancreatitis following the ERCP for more than 48 hours. This included 12 females. The mean age of the patients was 34 years. The indication for ERCP were choledocholithiasis in 5, recurrent acute pancreatitis in 12 patients, chronic pancreatitis in 1, acute pancreatitis with cholangitis in 1 and suspected Sphincter of Oddi dysfunction in 1. Balloon sphincterotomy was done in 7 patients and needle-knife sphincterotomy in 1 patient. 5 had mild, 13 had moderate and 2 had severe post ERCP pancreatitis. Median duration of hospital stay was 4 days range (3-16) days. Of these 2 patients with severe acute pancreatitis, associated cholangitis was noted in one and the other patient developed pancreatic fluid collection and ascites. Both of them improved with conservative management. There was no mortality. No prophylaxis for prevention of post-ERCP pancreatitis was given. Conclusion: Severe post-ERCP pancreatitis is not very common and usually has a good outcome. 


\section{Pancreatic Tumors - Basic}

\section{PT-B1}

\section{The Role of Cyclooxygenase-2 Gene Polymorphisms in Pancreatic Carcinogenesis}

\author{
R. Talar-Wojnarowska, A. Gasiorowska, M. Olakowski,
} P. Lampe, B. Smolarz, E. Malecka-Panas

Department of Digestive Tract Diseases, Medical University of Lodz, Poland; Department of Digestive Tract Surgery, Silesian Medical University, Katowice, Poland; Laboratory of Molecular Genetics, Lodz, Poland

Background: Cyclooxygenase- 2 (COX-2) is a key enzyme involved in many biologic processes, such as cell proliferation, invasion, metastases and angiogenesis, which are all relevant to cancer development and progression. The purpose of this study was to evaluate the clinical significance of $-765 \mathrm{G} / \mathrm{C}$ and $-1195 \mathrm{G} / \mathrm{A}$ COX-2 gene polymorphisms in patients with pancreatic cancer. Methods: The study included 201 patients: 85 with pancreatic cancer (PC) and 116 healthy controls. $-765 \mathrm{G} / \mathrm{C}$ and $-1195 \mathrm{G} / \mathrm{A}$ COX-2 genes polymorphisms have been studied in DNA isolated from blood samples. The associations of the analysed genotypes and clinical data at diagnosis have been evaluated. Results: We found an increased frequency of the homozygous -1195AA COX-2 genotype in patients with PC $(53.7 \%)$ compared with control group $(21 \%)(\mathrm{p}<0.01)$. In contrast, the distribution of genotype and allele frequencies of the $-765 \mathrm{G} / \mathrm{C}$ COX-2 polymorphism in the PC patients did not differ from those in control groups. The correlation between presence of homozygous -1195 AA COX-2 genotype and tumor size $>3 \mathrm{~cm}$ has been observed $(p<0.05)$. Analysed polymorphisms were unrelated to the patients' sex and age as well as with the presence of regional or distant metastases. Conclusion: These preliminary results indicate that $-1195 \mathrm{G} / \mathrm{A}$ COX-2 polymorphism may play important role in pancreatic carcinogenesis, however further studies are needed to investigate its possible association with PC prognosis.

\section{PT-B2}

\section{Abnormalities of Glucose Metabolism in Pancreatic Cancer}

\author{
G. Aggarwal, R. Pannala, R. Basu, A. Basu, S.T. Chari \\ Division of Gastroenterology and Endocrinology, Mayo \\ Clinic, Rochester, MN; Mayo Clinic, Scottsdale, Arizona, \\ USA
}

Background: Up to $85 \%$ of pancreatic cancer $(\mathrm{PaC})$ patients have either impaired fasting glucose or diabetes mellitus (DM) which is frequently new-onset ( $<36$ months) and resolves after tumor resection. We compared glucose metabolism between $\mathrm{PaC}$ patients and controls with varying degrees of hyperglycemia. Methods: We performed the Frequently Sampled Intravenous Glucose Tolerance Test
(FSIVGTT) and determined the parameters of insulin secretion and action using the MINMOD Millenium ${ }^{\circledR}$ model in $28 \mathrm{PaC}$ patients (mean age $69 \mathrm{yrs}, 18$ males) as well as 12 matched controls with normal fasting glucose (NFG) and 9 with impaired fasting glucose (IFG). Cross sectional analysis of $\mathrm{PaC}$ patients was done using the Kruskal Wallis test. Cases and controls were compared using the Wilcoxon ranks-sum test. Results: Among PaC patients, those with DM had the worst impairment in the acute insulin response to glucose [median, $3.4(-0.13)-59.2) \mathrm{mU} \mathrm{L}-1 \mathrm{~min}-1]$ and the highest insulin resistance [median, 3.2 (2-5.4) $\mathrm{mM} \mathrm{mU} \mathrm{L-2]} \mathrm{despite} \mathrm{losing} \mathrm{the} \mathrm{most} \mathrm{weight}$ [median, 18.2 (3.6-23.7) kg]. PaC patients with NFG when compared to NFG controls had higher beta cell function [median, 125 (102.9165.3) $\mathrm{mU} \mathrm{mM-1;}=0.006]$ in the face of more marked insulin resistance [median, 1.5 (1-1.8)mMmUL-2; $\mathrm{p}=0.003$ ]. Conclusions: $\mathrm{PaC}$ patients with DM have an impaired acute insulin response to glucose and marked insulin resistance despite profound weight loss. PaC patients with NFG have high insulin resistance with compensatory increase in beta-cell function as compared to NFG controls. These abnormalities in glucose homeostasis in $\mathrm{PaC}$ are likely due to tumor secreted diabetogenic substances.

\section{PT-B3 \\ K-ras and p16 Expression in Pancreatic Cancer Tissues and Correlation with Tumour Progression in Pancreatic Cancer}

\section{S.K. Polipalli, S.A. Husain, R. Gondal, A. Agarwal, P. Kar}

Department of Medicine, Maulana Azad Medical College; Department of Biosciences, Jamia Milia Islamia; Departments of Pathology and GI Surgery, G.B. Pant Hospital, New Delhi, India

Introduction: The activation of the proto-oncogene and inactivation of the tumour suppressor gene are key steps in the development of the human cancer. K-ras and p16 are examples of such genes respectively. Aim: In the present study, our aim was to determine the role of these genes in the carcinogenesis of pancreas by immunohistochemistry. Methods: Sections from paraffin embedded blocks of surgically resected specimens of pancreatic cancer (65 cases), chronic pancreatitis (50 cases), control pancreas (10 cases) were stained with the monoclonal antibody p16 and K-ras. Results were scored semi quantitatively and statistical analysis performed. P16 expression was scored as percentage of the nuclei stained. K-ras expression was scored as type of expression- membranous, cytoplasmic and nuclear staining. We have correlated with tumour invasiveness, differentiation and stage. Result: Over expression of p16 was seen in 54\% of pancreatic cancer cases and was not seen in chronicpancreatitis or in control pancreas. P16 expression in pancreatic cancer was significantly higher than in inflammatory or control pancreas $(p<0.0001)$. There was no correlation of $\mathrm{p} 16$ expression with tumour invasiveness, differentiation and stage. K-ras expression was positive in all the cases of pancreatic cancer, chronicpancreatitis and control pancreas cases. But expression pattern in chronicpancreatitis and control pancreas was focal and membranous; where as in case of pancreatic cancer membranous expression pattern was altered to cytoplasmic and nuclear, with higher intensity. No correlation of K-ras staining pattern with tumour invasiveness, differentiation and stage of pancre- 
atic cancer. Conclusion: K-ras and p16 expression is significantly higher and K-ras expression pattern is significantly different in pancreatic cancer than chronicpancreatitis and control pancreas. Expression does not correlate with tumour invasiveness, differentiation and stage.

\section{PT-B4 \\ N-nitrosobis (2-oxopropyl) Amine -induces Pancreatic and Hepatic Cancer in Syrian Hamsters}

\author{
J. Wei, J. Wu, Y. Zhang, H. Ren, J. Chen, F. Guo, C. Dai, \\ Z. Qian, Z. Xu, Y. Miao \\ Department of General Surgery, The First Affiliated \\ Hospital of Nanjing Medical University, China
}

Background: The Syrian golden hamster model of pancreatic cancer was reported in 1977 and is generally accepted as the best model available. Aim: to find some new value in this model. Methods: In the present study, hamsters were treated with $\mathrm{N}$-nitrosobis (2-oxopropyl) amine (BOP) once a week for 4 weeks $(20 \mathrm{mg} / \mathrm{kg}$, subcutaeous injection). Subsequently, hamsters were sacrificed every 2 weeks to determine the presence of neoplasms. Results: Hepatic neoplasms were found earlier than pancreatic tumors and were present in greater numbers. The incidence of pulmonary tumors was also higher than reported in other studies. Conclusion: These results indicate that further research is needed to better define and evaluate a BOP-induced pancreatic cancer model

\section{PT-B5 \\ Overexpression of ABCG2 Correlates with Invasion and Poor Prognosis in Pancreatic Cancer}

\author{
F. Wang, X. Xue, J. Wei, J. Wu, Y. Miao \\ Department of General Surgery, The First Affiliated \\ Hospital of Nanjing Medical University, China
}

Background: $\mathrm{ABCG} 2$ has been found to be strongly expressed in a variety of human epithelial cancers, and correlates with carcinogenesis and aggressiveness. It is unclear whether ABCG2 acts as a tumor metastasis promoting factor in human pancreatic cancer. Aims: This study aims to determine the prognostic value of ABCG2 in patients with pancreatic adenocarcinoma. Methods: We analyzed ABCG2 expression by immunohistochemistry in a retrospective cohort of 110 patients with resectable pancreatic ductal adenocarcinoma. The relationship between ABCG2 expression and the clinicopathological data of patients with pancreatic cancer were also evaluated. Survival data were assessed by Kaplan-Meier analyses. Results: ABCG2 overexpression was observed in 35 (32\%) of 110 pancreatic cancer patients. ABCG2 overexpression correlated with histological grade $(\mathrm{P}=0.000)$, tumor stage $(\mathrm{P}=0.031)$, stage (UICC; $\mathrm{P}=0.030)$, lymph node metastasis $(\mathrm{P}=0.021)$ and perineural invasion
$(\mathrm{P}=0.023)$. Multivariate analysis revealed that stage, lymph node metastasis and perineural invasion were independent prognostic factors, but ABCG2 overexpression was not (risk ratio, 1.118; 95\% CI, $0.552-2.268, \mathrm{P}>0.05)$. By Kaplan-Meier analysis, patients whose pancreatic cancer cells overexpressed ABCG2 (median survival, 5 months) had a significantly worse prognosis than patients without ABCG2 overexpression (median survival, 13 months; log-rank, $\mathrm{P}<0.01$ ). Conclusions: These novel findings suggest that high expression of ABCG2 by pancreatic cancer cells is significantly associated with poor prognosis by promoting tumor metastasis and invasion, which may be a useful target in the development of future targeted treatments.

\section{PT-B6 \\ Galectin-1 Secreted by Activated Stellate Cells in Pancreatic Cancer Stroma Promotes Proliferation and Invasion of Pancreatic Cancer Cells}

X. Xue, Z. Lu, K. Jiang, O. Li, Y. Miao

Department of General Surgery, The First Affiliated

Hospital of Nanjing Medical University, China

Background: Pancreatic stellate cells (PSCs) as part of tumor microenvironment may play an important role in the progression of pancreatic ductal adenocarcinoma (PDAC). Aims: To clarify the activated PSCs are the origin of the highly expressed Galectin-1 in the stroma of PDAC tissue. And to evaluate the effect of the secreted Galectin-1 on proliferation and invasion ability of pancreatic cancer cell lines in vitro. Methods: We conducted this research based on primary culture of the PSCs. Immunohistochemistry study, Western Blot and RT-PCR were carried out to check the cellular origin of Galectin-1 in PDAC tissue. By utilizing modified Boyden chamber, we established in vitro coculture system of PSCs with pancreatic cancer cells (PCCs), and based on which we assessed the proliferation and invasion ability of PCCs with or without Galectin-1 antagonists. Results: We identified PSCs as the primary source of the highly expressed Galectin-1 in PDAC stroma. Galectin-1 secreted by PSCs increased PCCs number in MTT assay and facilitated PCCs infiltration in invasion assay. Conclusion: Under malignant circumstances, PSCs express and secret Galectin-1, which can promote proliferation and invasion ability of PCCs. 


\section{PT-B7 \\ The Transcriptional Regulation of Cancer Stem Cell-related Gene ABCG2 by the Homeobox Gene MSX2}

\section{S. Hamada, K. Satoh, M. Hirota, A. Kanno, A. Masamune, K. Kikuta, K. Kume, J. Unno, T. Shimosegawa \\ Division of Gastroenterology, Tohoku University Graduate School of Medicine, Sendai, Miyagi; Division of Clinical Research, Miyagi Cancer Center Research Institute, Natori, Miyagi, Japan}

Background: Pancreatic cancer has high mortality, due to the resistance against the conventional therapy. Recent researches revealed the contribution of cancer stem cells to the chemoresistance. Previously, we confirmed that the homeobox gene MSX2 enhances epithelial mesenchymal transition of pancreatic cancer cells, which is also the cancer stem cell phenotype. Aim: To clarify the regulatory mechanism of stem cell-related gene ABCG2 in pancreatic carcinoma cells. Methods: The gene expression profiles of the MSX2 overexpressing cells and control cells were compared by microarray. The induction of ABCG2 was confirmed by real-time RT-PCR and flow cytometry. Chemosensitivity of each cell line was confirmed by MTT assay. The transcriptional regulation of ABCG2 was assessed by luciferase assay and ChIP assay. Results: ABCG2, which is a cancer stem cell-related transporter gene was identified as significantly upregulated in MSX2 over-expressing cells. In contrast, ABCG2 expression level was decreased in MSX2 knockdown cells. Chemoresistance of these cells correlated with the expression levels of MSX2 and ABCG2. Knockdown of ABCG2 resulted in re-sensitization to chemotherapeutic agents. Furthermore, MSX2 turned out to regulate ABCG2 expression via the SP1 binding site, independently of the MSX2 binding site. Conclusion: MSX2 regulates chemoresistance of pancreatic cancer cells by affecting the ABCG2 expression. This regulatory mechanism will provide novel therapeutic target against the pancreatic cancer stem cells.

\section{PT-B8 \\ Role of Pancreatic Stellate Cells in Pancreatic Cancer: The Urokinase Plasminogen Activator System}

\author{
E. Fiala-Beer, Z. Xu, P. Phillips, L. Yang, D. Goldstein, \\ R. Pirola, J. Wilson, M. Apte
}

Pancreatic Research Group, University of New South Wales, Sydney, Australia

Background: The Urokinase Plasminogen Activator (uPA) system [comprising urokinase plasminogen activator (uPA), tissue plasminogen activator (tPA), the uPA receptor (uPAR), and the plasminogen activator inhibitors (PAI-1 and PAI-2)] has been implicated in cancer progression. However, it is not fully characterised in pancreatic cancer (PC), particularly in the stromal reaction of PC. We have shown that pancreatic stellate cells (PSCs) produce the stromal reaction of $\mathrm{PC}$ and facilitate $\mathrm{PC}$ progression by inducing tumour growth and metastasis. Aims: To i) identify uPA system components in human PSCs (hPSCs) and in MiaPaCa2 (human PC cells); ii) assess the effect of hPSCs on uPAR expression in MiaPaCa2; and iii) assess whether hPSC-induced $\mathrm{MiaPaCa} 2$ proliferation is modulated by the uPA system. Methods: i) hPSCs isolated from resected PC tissue (cancer associated hPSCs, CAhPSCs). mRNA and protein for uPA system assessed by qRT-PCR and immunoblotting respectively. ii) UPA system mRNA levels assessed in MiaPaCa2 cells after coculture with CAhPSC. iii) $\mathrm{MiaPaCa} 2$ proliferation assessed after incubation with CAhPSC secretions \pm amiloride (uPA inhibitor). Results: i) CAhPSCs express mRNA and protein for uPA, tPA, uPAR, PAI-1 and PAI-2. MiaPaCa2 exhibit mRNA for all uPA components. ii) CAhPSCs induce uPAR mRNA in MiaPaCa2 (1.87 fold, $\mathrm{p}<0.05, \mathrm{n}=4$ separate CAhPSC preparations). iii) Amiloride significantly inhibits CAhPSC-induced MiaPaCa 2 proliferation by $23 \pm 4.4 \%$ $(\mathrm{p}<0.05, \mathrm{n}=3$ separate CAhPSC secretions). Conclusions: This is the first demonstration of uPA system expression in hPSCs. Notably, hPSCs induce uPAR expression in PC cells and hPSC-stimulated PC cell proliferation is mediated in part via uPA. Implication: Modulation of the uPA system may help to disrupt stromal-tumour interactions and inhibit PC progression.

\section{PT-B9}

\section{Heat Shock Factor-1: Therapeutic Target for Pancreatobiliary Tumors}

V. Dudeja, R. Chugh, V. Sangwan, N. Mujumdar, R. Dawra, S.M. Vickers, A. Saluja

Division of Basic and Translational Research, Department of Surgery, University of Minnesota, Minneapolis, MN, USA

Background: Heat Shock Factor 1 (HSF1) is a transcription factor for multiple cell survival proteins like HSP70. We have previously shown that heat shock protein 70 plays a pro-survival role in pancreatic cancer pathogenesis. Since HSF1 regulates multiple survival proteins including HSPs, we hypothesize that overexpression of HSF1 is vital for the survival of pancreatobiliary tumors and inhibition of its expression should induce apoptosis in pancreatiobiliary tumor cells. Methods: HSF1 expression was reduced in pancreatic (MiaPaCa-2 and S2013) and cholangiocarcinoma (KMCH, KMBC) cell lines by treatment with HSF1 siRNA. Two unique sequences of HSF1 siRNA were used to rule out any off-target effects of siRNA. Cell viability (MTT assay) and apoptosis (annexin V staining, caspase 3 and 9 and TUNEL) were measured. Results: Inhibition of HSF1 expression by HSF1siRNA markedly reduced the viability of all the cancer cell lines at $96 \mathrm{~h}$. Viability (\% of control) expressed as mean \pm SEM: MiaPaCa-2: $37.2 \pm 3.2, \mathrm{KMCH} 41.3 \pm 7.61$, data not shown for S2013 and KMBC. Inhibition of HSF1 expresion also led to increased annexin V staining and TUNEL positivity in all the cell lines studied (data not shown). Further, inhibition of HSF1 expression also led to activation of caspase-3 in all the cancer cell line studied. Caspase 3 (\% of control) expressed as mean \pm SEM: MiaPaCa- 2 : 497.4 \pm 24.3 ; KMCH-465.2 \pm 22.68 ; data not shown for S2013 and KMBC. Similar results were observed when the HSF1 expression was inhibited by the other HSF1siRNA sequence. Conclusion: Silencing of HSF1 expression activates caspase depen- 
dent apoptotic cell death in pancreatobiliary cancer cells. Thus HSF1 holds a great promise as a potential candidate for the drug development.

\section{PT-B10}

\section{Understanding the Cross Talk Between $\beta$ Catenin and NFk $\beta$ in Chronic Pancreatitis and Pancreatic Cancer}

\author{
S. Kumaran, J. Venkataraman, D. Halagowder \\ Department of Biotechnology, University of Madras; \\ Department of Gastroenterology, University of Madras, \\ Chennai, India
}

Background: The molecular basis of pancreatic cancer is not well understood. Chronic pancreatitis is an inflammatory disease of the pancreas that is associated with gradual organ damage and an increased risk of developing cancer. A detailed study of the chronic inflammatory processes in the pancreas may provide insights into the understanding of the progression of pancreatic cancer. NF- $\mathrm{kB}$, a transcription factor, plays an important role in the regulation of inflammatory responses as well as in carcinogenesis. NF- $\kappa \mathrm{B}$ has been reported to have pro- and anti-apoptotic effects depending on the stimulus. It promotes cell cycle progression by regulating the expression of C-myc gene. It has been reported that C-myc plays an important role in cell proliferation. $\mathrm{NF}-\kappa \mathrm{B}$ has been implicated as a transcriptional activator of proapoptotic genes, such as Fas and FasL. But the mechanism by which $\mathrm{NF \kappa B}$ induces cell proliferation and escapes from the apoptotic pathway in pancreatic cancer is not clear. $\beta$ catenin plays an important role in development and homeostasis. The interaction between $\beta$ Catenin and NFKB has not been studied in chronic pancreatitis and pancreatic cancer. Aim: To understand the cross talk between $\beta$ Catenin and $\mathrm{NF} \kappa \mathrm{B}$ in chronic pancreatitis and pancreatic cancer. Methods: Pancreatic tissue samples were collected from consecutive patients undergoing surgery for chronic pancreatitis (Frey's or Beger's) and pancreatic malignancy (Whipple's procedure). Normal samples were collected from 7 patients undergoing palliative surgery for advanced gastric carcinoma. Samples were collected under the approved protocol by the Institutional Ethical Review Board. Study population consisted of 29 patients with pancreatic cancer, 47 patients with chronic pancreatitis and 7 with normal pancreas. Patients with CECT evidence of chronic pancreatitis and histology proven adenocarcinoma of the pancreas were included in the study. Expression of $\beta$ Catenin and NFкB was assessed by Immunohistochemistry and Western blot. Co.immunoprecipitation was used to understand the interaction between $\beta$ Catenin and NFKB. Fas and C-myc gene expression was assessed by RT PCR. Results: There were 32 men and 15 women with chronic pancreatitis (Gp I) and 20 men and 9 women with pancreatic cancer (Gp II). The mean age for men and women was 35 and 50 in Gp I and II respectively. In chronic pancreatitis, 17 out of $32(53 \%)$ men were smokers and 23 out of $32(72 \%)$ were consuming alcohol. In pancreatic cancer 11 out of 20(55\%) men were smokers and 14 out of 20 (70\%) were alcoholic. $\beta$ Catenin was over expressed in 19 out of 47 (40\%) patients in chronic pancreatitis (Gp I) and 17 out of $29(58 \%)$ patients with pancreatic cancer (Gp II) compared with normal pancreas (Gp III). NFkB was over expressed in two-thirds of patients in both Gp I and Gp II. Over expressed $\beta$ Catenin formed a complex with NFKB in 10 out of 47 patients (21\%) in Gp I and 16 out of 29 patients (55\%) in Gp II. Down regulation of Fas gene was observed in 9 out of 47 patients (19\%) in Gp I and 16 out of 29 (55\%) in Gp II patients. There was up regulation of C-myc gene in 12 out of 47 $(25 \%)$ in GP I patients and 17 out of 29 (58\%) in Gp II patients. Overall, more than $50 \%$ of patients showed similar expressions were considered as significant. Conclusion: Increased expression of $\beta$ Catenin possibly disrupts the balance between cell proliferation and apoptosis by interacting with $\mathrm{NF} \kappa \mathrm{B}$ and recruiting it into the cell survival pathway alone in most patients with pancreatic cancer and in fewer cases of chronic pancreatitis. One can hypothesis that the cross talk between $\beta$ Catenin and NFKB could serve as a marker for progression of chronic pancreatitis to pancreatic cancer.

\section{PT-B11 \\ Gene Expression Profiling Reveals Long Intronic Non-Coding RNAs Differentially Expressed in Pancreatic Cancer and Metastasis}

M.C.C. Machado, A.C. Tahira, M.S. Kubrusly, M.F. Faria, S. Verjovski-Almeida, E.M. Reis

Department of Gastroenterology and Biochemistry, University of Sao Paulo, Brazil

Aim: To investigate the expression of noncoding RNAs (ncRNAs) in clinical samples from pancreatic cancer patients. Introduction: Pancreatic Adenocarcinoma (PA) is known by its aggressiveness and poor prognosis. Therefore, new molecular markers for detection and prognosis are needed. Long ncRNAs expressed from intronic regions play different roles in regulation of gene expression, but its relevance in pancreatic cancer has not been investigated. Methods: Spotted cDNA microarrays interrogating ncRNAs were used to profile RNA samples from 15 primary tumors (T), 8 chronic pancreatitis (CP), 9 non-tumor pancreatic tissue $(\mathrm{N})$ and 6 biopsies from distant metastasis (M). Results: We identified 142 transcripts differentially expressed in T relative to $\mathrm{N}$ and $\mathrm{CP}$ samples (FDR $<10 \%)$ comprising 37 ncRNAs. Comparison between $\mathrm{M}$ to $\mathrm{T}(\mathrm{FDR}<5 \%)$ showed a signature of 143 transcripts, including 45 ncRNAs, revealing transcripts possibly associated with the invasive phenotype. Gene enrichment analysis indicated that the metastasis signature is enriched in ncRNAs transcribed from intronic regions of genes from the MAPK pathway and involved in apoptosis, being 9 transcripts in total. Increased expression in metastatic samples of intronic ncRNAs mapping to the PPP3CB and MAP3K14 loci was confirmed by qPCR. Conclusion: Our work revealed a gene expression profile correlated to metastasis and pancreatic cancer that is enriched in long intronic ncRNAs. Further investigation is warranted to determine the functional role played by these transcripts during transformation and progression of pancreatic tumors. 


\section{PT-B12}

Minnelide as a Potent Chemotherapeutic Agent Against Pancreatic Cancer

\author{
V. Sangwan, R. Chugh, V. Dudeja, S.M. Vickers, A. Saluja \\ Division of Basic and translational Research, Dept of \\ Surgery, University of Minnesota, Minneapolis, USA
}

Background: Pancreatic cancer carries one of the most dismal prognosis, with a median survival of less than six months. Previous data from our group has shown that triptolide, a diterpenoid, inhibits HSP70 expression leading to cell death in pancreatic cancer cells, as well as growth and loco-regional spread of pancreatic tumors in vivo. However, limitations arising from the insolubility of triptolide in water have delayed its progression into the clinic. We therefore developed a water-soluble prodrug of triptolide named Minnelide which converts to the parent compound both in vitro and in vivo. Aim: To evaluate the efficacy of Minnelide as a potential therapeutic agent for pancreatic cancer in vivo. Methods: Pancreatic cancer cells of varying metastatic potential (MIA PaCa-2/ S2-013/ Aspc-1) were implanted into the pancreas of nude mice, which were treated with either vehicle or Minnelide at different stages of tumor formation. Results: In the MIA PaCa-2 in vivo orthotopic model, Minnelide dramatically decreased tumor size and loco-regional tumor spread, and extended survival, both in the early ( $7 \mathrm{~d}$ post-inoculation; $>83 \%$ decrease in tumor volume vs. control) and late ( $28 \mathrm{~d}$ post-inoculation; $>70 \%$ decrease in tumor volume vs. control) stages of pancreatic cancer. In the metastatic S2013 orthotopic mouse model, tumor volume decreased to $>75 \%$ vs. control. Significantly, in the metastatic Aspc-1 orthotopic model, median survival of saline-injected mice was 36 days, whereas Minnelide-treated mice are tumor-free for at least 186 days. In another study, in the Aspc-1 mouse model, Minnelide treatment was started when the first saline-treated mouse was euthanized due to excess tumor burden. We are very excited to report that 21 days post-treatment, the tumors in these mice are no longer palpable. Conclusion: Minnelide holds great promise as a novel single-agent therapeutic strategy against pancreatic cancer. It is a potent, non-toxic anti-cancer agent, and not only prevents tumor progression, but also causes tumor regression in late stage pancreatic tumors. We are currently in process of initiating Phase I clinical trials for this drug.

\section{PT-B13}

\section{Environmental Induced Pancreatic Cancer: Etiologic Clues from Genetic and Epigenetic Analysis}

\author{
S. Varshney, R. Sekwani, R. Varshney, K.K. Maudar, \\ G. V. Raghuram, P. K. Mishra \\ Bhopal Memorial Hospital and Research Center, Bhopal, \\ India
}

Aim: Chronic pancreatitis and pancreatic cancer, like many other complex diseases, have genetic and environmental components to their etiology. Only $20 \%$ of pancreatic cancers are amenable to surgical resection at presentation, as diagnosis is late and cancer has often metastasized. Little is known about its etiology. Although tobacco is the only established cause, a large proportion of cases are still due to unrecognized factors. It has been suspected that environmental chemicals such as $N$-nitroso (carbamates) compounds, either from endogenous or exogenous sources, are among the etiologic agents in pancreatic cancer development. Henceforth, it is biologically plausible that both genetic and epigenetic factors play an important role in isocyanates, reactive components of carbamate pesticides, mediated pancreatic carctnogenesis. Methods: We examined the molecular mechanism underlying genetic and epigenetic regulations in isocyanate induced pancreatic adenocarcinoma in cultured normal human pancreatic cells in cultured normal human pancreatic cells using $N$-succinimidyl $N$ methylcarbamate, a chemical entity that mimics the effects of isocyanate and un vitro conditions. Results: The isocyanates induced genetic alteration, resulting in extensive DNA damage, evidenced by increases in ATM, ATR, YH2AX, and p53 expression levels. While chromosomal abnormalities and variable amplification of inter-simple sequence repeats in treatedcells, elevated levels of K-ras, P21, COX-2 proteins, hyper and hypomethylation of $\mathrm{CpG}$ islands for tumour suppressor genes $\mathrm{p} 21$ and $\mathrm{p} 16$ promoters respectively, further demonstrate isocyanate-induced epigenetic alterations. Conclusion: The results obtained would be instrumental in predicting which patients would benefit from surgical intervention and or chemotherapy upon ex-vivo correlation.

\section{Pancreatic Tumors - Clinical}

\author{
PT-C1 \\ Laparoscopic Common Hepatic Artery \\ Ligation and En-bloc Resection of Celiac \\ Artery with Distal Pancreatectomy for \\ Advanced Pancreatic Cancer \\ V. Raut, K. Takaori, Y. Kawaguchi, M. Mizumoto, \\ M. Kawaguchi, M. Koizumi, S. Uemoto \\ Department of HPB Surgery and Transplantation, \\ Graduate School of Medicine, Kyoto University, Kyoto, \\ Japan; Department of HBP Surgery and Liver \\ Transplantation, Hospital Beaujon, University Denis \\ Diderot, Paris, France
}

Background: Pancreatic body carcinomas are asymptomatic and usually progress to advanced stage and involve major arteries. Resection of advanced cancer along with en-bloc resection of common hepatic artery and celiac trunk enables 'curative' resections and only possible available treatment. However, celiac axis resection always has risk of compromising blood supply to liver resulting hepatic insufficiency. Aim: We evaluated practicability of a twostage procedure for advanced pancreases body cancer, laparoscopic clamping of common hepatic artery followed by open distal pancreatectomy with en-bloc celiac arterial resection to prevent hepatic insufficiency. Method: 75-year-old woman, diagnosed with $50 \mathrm{~mm}$ pancreatic body mass, invading splenic artery, common hepatic artery, splenic vein, and portal vein at confluence. Stage-1: At laparoscopy, 
after confirming absence of peritoneal and superficial liver metastases and negative peritoneal cytology; we approached common hepatic artery through lesser sac and ligated. Stage-2: Her liver function tests were normal after two weeks, and CT angiography showed complete blockage of common hepatic artery with sufficient collateral circulation to liver through inferior pancreatico-duodenal artery and gastroduodenal artery. We performed open distal pancreatectomy with en- bloc resection of celiac artery. Histopathology examination confirmed R0 resection. Discussion: Celiac axis resection with distal pancreatectomy improves the chance of R0 resection and potentially, survival of patient. Preoperative laparoscopic common hepatic artery ligation is safe, effective, and in-expensive technique to prevent postoperative hepatic insufficiency and improves safety of en-bloc celiac artery resection with distal pancreatectomy. Also these patients have high risk of peritoneal dissemination. Diagnostic laparoscopy is useful to detect occult metastasis, which are missed by per-operative CT scan.

\section{PT-C2}

\section{Pilot Trial of Wilms-Tumor-1 (WT1) Peptide Vaccine Therapy for Chemorefractory Advanced Pancreatic Cancer}

\section{S. Egawa, T. Okada, H. Hayashi, Y. Kitamura, M. Unno \\ Division of Hepato-Biliary-Pancreatic Surgery, Tohoku University, Japan}

Aim: Peptide vaccine immunotherapy is an old-new modality for cancer. Wilms-Tumor-1 (WT1) gene product is a promising tumor associated antigen and expressed in $67 \%$ of pancreatic cancer. Since there is no established treatment for chemorefractory pancreatic cancer, we conducted a pilot study for WT1 peptide vaccine therapy combined with preceding chemotherapy to see the safety and effectiveness (UMIN 000001515). Methods: Patients with HLA-A2402 (60\% in Japanese) who had progressive disease after treated with gemcitabine (GEM), TS-1, or GEM/S1 were enrolled if performance status (PS) was $0-1$. GMP grade WT1 peptide (3mg/visit) was injected with incomplete Freund adjuvant intradermally in bilateral axillary, abdominal and inguinal regions for at least eight visits. One of the preceding chemotherapy was combined with vaccination. Primary endpoint is safety and disease progression, secondary endpoint is overall survival and induction of WT1 specific immune reaction. Results: Total 27 patients with metastasized or locally advanced pancreatic cancer were enrolled. Twelve patients had recurrence after resection. Fourteen patients received chemo-vaccination as second line, and 13 as third line. Dermal reaction at the injection site was less than Grade 1. Adverse effect greater than Grade 3 occurred in three patients (thrombosis of superior mesenteric artery, stress cardiomyopathy and thrombocytopenia), which relation with the vaccination is unknown. Overall median survival time of 27 patients is 5.0 months. Patients with PS0 survived significantly longer $(8.0 \mathrm{M})$ than PS1 $(3.0 \mathrm{M})$. Conclusion: Average median survival after initiation of second line chemotherapies is six months in literature review. Chemoimmunotherapy using WT1 peptide vaccine can be a promising treatment for the patients with advanced pancreatic cancer in good PS.

\section{PT-C3 \\ Periampullary Carcinoma in a Patient with Dorsal Agenesis of Pancreas}

\author{
A. Kapoor, P. Sihag, R.K. Singh \\ Department of Surgical Gastroenterology, Sanjay Gandhi \\ Postgraduate Institute of Medical Sciences, Lucknow, \\ Uttar Pradesh, India
}

Introduction: Dorsal agenesis is a rare developmental anomaly of the pancreas. Till date only about 50 cases have been reported in literature. We here report a case of periampullary carcinoma with dorsal agenesis of pancreas. Case Report: A 55 year old male patient presented with painless progressive obstructive jaundice for 3 months. ERCP showed a ulceronodular growth at the papilla and preoperative biliary stenting was done for cholangitis. CT scan of abdomen showed a periampullary tumour but the body and tail of pancreas were absent. At surgery, there was a $2 \times 1 \mathrm{~cm}$ mass in region of ampulla and further dissection confirmed the CT findings. The body and tail of the pancreas was absent and the superior mesenteric-splenic vein junction was bare. A pylorus preserving pancreaticoduodenectomy (entailing a total pancreatectomy in this patient) was done and reconstruction comprised only hepaticojejunostomy and duodenojejunostomy. The specimen was studied with a pancreatogram and only a single main ventral pancreatic duct with prominent side branches was demonstrated. Absence of dorsal ductal system and minor papilla established complete agenesis of dorsal pancreas. Histopathology revealed a periampullary adenocarcinoma infiltrating into pancreas, all resection margins and lymph nodes were free. Postoperative course was uneventful and patient was discharged on insulin and pancreatic enzyme replacement therapy. Literature review revealed that agenesis of the dorsal pancreas may be partial (more frequent) or complete. In complete agenesis, the minor papilla, accessory pancreatic duct, body and tail of pancreas are absent and pancreatogram is essential for diagnosis. Conclusion: Pancreaticoduodenectomy in patients with dorsal agenesis entails a total pancreatectomy. Preoperative counseling is necessary because patients develop insulin dependent diabetes with exocrine insufficiency and require life-long replacement after pancreaticoduodenenctomy.

\section{PT-C4 \\ Well-differentiated Pancreatic Endocrine Tumors: Which Operative Risk? for Which Survival? \\ R. Cherif, S. Gaujoux, A. Couvelard, S. Dokmak, B. Aussilhou, Ph. Ruszniewski, J. Belghiti, A. Sauvanet \\ Departments of HBP Surgery, Pathology and Gastroenterology, Hôpital Beaujon, University Paris VII, France}

Aim: Well-differentiated pancreatic endocrine tumors (PET) are usually resected even if nonfunctioning or metastatic to the liver. The aim of this study was to assess factors influencing disease-free survival after PET resection according to presentation. Methods: From 1995 to 2008, 118 patients (median age 51 years) underwent PET 
resection (37 pancreaticoduodenectomies [PD], 43 distal pancreatectomies [DP], 27 enucleations [EN], 10 medial pancreatectomies [MP] and 1 total pancreatectomy), associated with liver metastases (LM) in $21(18 \%)$, resected in 19(16\%). Disease-free survival (DFS) was evaluated using Kaplan-Meier and compared with Log rank test. Results: $83 \%$ of PET were sporadic and $75 \%$ nonfunctioning. Postoperative mortality was $1.7 \%$ (1 EN, $1 \mathrm{MP}$ ). According to WHO classification, $24 \%$ were benign lesions (B), $25 \%$ of uncertain behavior (UB) and $51 \%$ carcinomas $(\mathrm{N}+=45, \mathrm{M}+=21)$. After a median follow-up of 51.5 months, 5-year DFS was $72 \%$ ( $B=100 \%$, UB $=94 \%$ and carcinoma $=57 \%$ ). Recurrence in UB tumors occurred in 4 of 5 cases after the 7 th year. Of the $32(72 \%)$ nonfunctional, $<$ or $=$ to $2 \mathrm{~cm}$, without radiologic metastases PET, 23 (72\%) were B, 6 (19\%) were $\mathrm{UB}$ and $3(9 \%)$ were carcinoma $(\mathrm{N}+)$. For this group, the 5 and 10 years DFS was $100 \%$ and $80 \%$ respectively ( 2 recurrences at 85 and 130 months). Conclusions: Nonfunctioning tumors $<$ or $=$ to $2 \mathrm{~cm}$ are malignant in only $9 \%$ of cases and their recurrences are rare and late. Since postoperative mortality is not nil even if after limited resections, the benefit/risk balance should be precisely discussed case by case. In other presentations, surgery is justified by a low mortality and a significant risk of tumor recurrence.

\section{PT-C5 \\ Chronic Calcific Pancreatitis with Carcinoma Pancreas: Survival After Resection}

R.S. Sindhu, T.U. Shabeerali, R. Subhash, M.L. Arunkumar, V.A. Iyoob, B. Natesh, N. Subhalal, R. Rajan, A.P. Kuruvilla, N. Lailaraji, B. Krishna

Departments of Surgical Gastroenterology and Pathology, Medical College, Thiruvananthapuram, India

Background: Literature regarding the survival of patients after resection for carcinoma in Chronic Calcific Pancreatitis (CCP) is scanty. Aim: To compare overall survival (OS) after resection, in CCP with carcinoma pancreas (CCP Ca) and denovo ductal adenocarcinoma pancreas (denovo $\mathrm{Ca}$ ). Methods: A prospective, single centre study was done to compare the OS of patients with $\mathrm{CCP} \mathrm{Ca}$ (pathologically proven) and denovo $\mathrm{Ca}$ underwent resection during the same period (from January 2003 to December 2009), excluding the cases with 30 days post-operative mortality, periampullary carcinoma and CCP dysplasia. Clinical and imagiological 6 monthly follow-up was done. Age, stage, differentiation, margin status and comorbidities were studied. Kaplan-Meier curve and other relevant statistical tests were done using PASW 18 version. Results: 62 patients with CCP Ca and 43 with denovo $\mathrm{Ca}$ underwent resection, of which, 25 (40.3\%) and 18 (41.8\%) respectively were lost to follow-up. Among those with followup 2 year OS was $55.5 \%$ and 5 year OS was $17.7 \%$ in CCP Ca, while it was nil in denovo $\mathrm{Ca}$. On multivariate analysis, 2 groups were comparable in $\mathrm{R} 1$ resections (CCP Ca: $13.5 \%$, denovo $\mathrm{Ca}: 26.9 \%, \mathrm{p}>0.276$ ) and anaesthetic risk, although the age (CCP Ca mean 45.4 years, denovo Ca mean 54.1 years, $\mathrm{p}<0.022$ ) and diabetic status (CCP Ca: $64.9 \%$, denovo Ca: $92 \%, \mathrm{p}<0.01)$ differed. Lymph node metastasis was $53.84 \%$ in denovo $\mathrm{Ca}$ and $27.02 \%$ in $\mathrm{CCP} \mathrm{Ca}(\mathrm{p}<0.042)$. Well differentiated tumours accounted for $86.48 \%$ in CCP Ca but only for $46.15 \%$ in denovo $\mathrm{Ca}(\mathrm{p}<0.003)$. Conclusion: Patients with CCP Ca have a better overall survival than denovo $\mathrm{Ca}$ pancreas following resection.

\section{PT-C6 \\ Radiofrequency Ablation of Unresectable Pancreatic Carcinoma: 10 Year Experience from Single Centre}

\author{
V. Singh, S. Varshney, A. Sewkani, R. Varshney, \\ G. Deshpande, P. Shaji, A. Jat
}

Department of Surgical Gastroenterology. Bhopal

Memorial Hospital and Research Centre, Bhopal, Madhya

Pradesh, India

Background: Radiofrequency ablation (RFA) is a local ablative method used for the palliative treatment of solid tumors. Its use in the ablation of liver tumours is well established. However, RFA of locally advanced pancreatic cancer as a cytoreductive means is a new treatment option with only a few studies found in the medical literature. We present our experience over ten years regarding feasibility, efficacy, safety and complications of radiofrequency ablation in unresectable pancreatic cancer. Methods: Our study includes 11 patients from Bhopal Memorial Hospital and Research Centre in whom RFA was performed for locally advanced unresectable pancreatic tumor but without other systemic spread of tumor from May 2001 to October 2010. Technique/Machine; Reason for unresectability: We used a Berchtold generator (Tuttlingen, Germany), generating 40-60 W of power. Radiofrequency needle was placed in the centre of tumor either CT guided (one patient) or intraop USG guided (ten patients). Approximately $4200 \mathrm{~W}$ of energy was delivered to tumor using saline perfused needle with the aim of producing a tumor necrosis of $3 \mathrm{~cm}$. intraoperative monitoring of tumor necrosis was done with ultrasound. Reason for unresectability was encasement and infiltration of portomesentric axis (superior mesenteric vein/artery or portal vein involvement) in all patients. In addition one patient had history of stroke and ischemic heart disease rendering her unfit for major operative resection (first patient of index study, underwent CT guided RF ablation). Results: Eleven patients between ages 45-72 years with histologically proven unresectable cancer of pancreas were included in the study. The tumor size ranging from $5-8 \mathrm{~cm}$ (mean $6.5 \mathrm{~cm}$ ). Ten patients underwent radiofrequency ablation during an open operation while one patient had percutaneous CT guided radiofrequency ablation. Endobiliary stenting for obstructive jaundice was done in all the patients. Surgical bypass was not done in any patient. Partial necrosis (up to $3 \mathrm{~cm}$ ) of the tumor was achieved in all cases. There was no major procedure related morbidity or mortality. One patient developed a $2 \mathrm{~cm}$ asymptomatic pseudocyst which was seen on follow up ultrasonography. Two patients developed self limiting pleural effusion and in two other patients there was transient ascites (with normal ascitic fluid amylase). Mild abdominal pain was seen in 5 patients. Neither of these patients required any treatment. One patient died of massive myocardial infarction one month after RFA. The survival of rest 10 patients ranged between 9 to 36 months. Eight patients had post RFA chemotherapy. Conclusion: RFA is a safe and effective technique for cytoreduction $(3 \mathrm{~cm})$ of unresectable pancreatic tumors. However, a larger study or randomized control trial is required to ascertain whether this can improve survival and quality of life in unresectable pancreatic cancer along with adjuvant chemotherapy. 


\section{PT-C7}

\section{A Registry for Patients with Pancreatic Cystic Tumours: Experience and Clinical Outcomes}

\author{
S. Sanyal, A.J. Sheen, A.K. Siriwardena \\ Hepatobiliary Surgery Department, Manchester Royal \\ Infirmary, UK
}

Introduction: Pancreatic mucinous cystic tumours are rare and carry a risk of malignant transformation. We report a clinical registry to ensure optimal staging and also to assist subsequent clinical management. Methods: Forty-nine patients with non- inflammatory pancreatic cystic lesions presenting to our regional hepato-pancreatobiliary clinic over an 8 year period from 2002 to present were included [29 females; mean age at presentation 67 (range 40-89 years)]. These patients have been followed up for an average of 24 months (range 1-102 months). There were 25 (51\%) asymptomatic patients, 19 $(39 \%)$ presented with abdominal pain and $5(10 \%)$ presented with other symptoms. Work-up included contrast-enhanced CT in 49 $(100 \%)$, EUS in $36(73 \%)$, EUS-FNA cytology in $30(61 \%$ of total or $83 \%$ of EUS pts). Median (range) cyst size was $37(11-130) \mathrm{mm}$. Twenty nine $(59 \%)$ of lesions were located in the head of the pancreas, with 8 patients having multiple cysts. There were a total of 17 branch duct IPMN, 12 main duct IPMN, 6 patients had serous cystadenoma (SCA), 6 patients had IPMN associated cancer, 3 had mucinous cystic neoplasm $(\mathrm{MCN})$ and 7 had other lesions. Of the 49 patients, 33 were managed conservatively, while 16 underwent surgery. Results: 16 patients were managed surgically on the basis of their staging tests ( 7 main-duct IPMN, 5 cystic adenocarcinoma, 2 branch-duct IPMN and 2 other). The remaining 33 were managed non-operatively. Cystic lesions included 15 branch duct IPMNs, 5 main duct IPMN, 5 SCAs, 2 MCNs and 3 suspected pseudocysts. Of the 5 main duct IPMN, 2 declined further intervention and 3 were not fit for surgery. During follow up (average 16.3 months [range 3-91 months]), interval restaging was undertaken in all and there have been no interval cancers. Conclusion: Caution must be exercised in extrapolating from small volume series; however these data suggest that provided careful staging is undertaken, selected patients with pancreatic cystic tumors can be managed without resection.

\section{PT-C8 \\ What Is the Role of Staging Laparoscopy in the Diagnosis and Staging of Periampullary Tumors?}

\author{
V. Bansal, M.C. Misra, P. Garg, S. Kapoor, A. Kumar, \\ A. Krishna \\ Departments of Surgery and Gastroenterology, All India \\ Institute of Medical Sciences, New Delhi, India
}

Background: Majority of patients with periampullary tumors have advanced disease at the time of diagnosis. Only $10-20 \%$ of the patients can undergo curative resections. Accurate preoperative staging decreases a lot of morbidity and mortality in these patients. Variety of preoperative imaging modalities like contrast enhanced computed tomography (CECT), magnetic resonance imaging (MRI), endoscopic ultrasound (EUS) and PET CT have been performed for accurate staging of these tumors. Although all these imaging modalities are sensitive in picking up tumors and vascular invasion but they are unable to pick small peritoneal and hepatic metastasis. Staging laparoscopy plays a role in detecting imaging occult metastasis. This prospective study was conducted to find out sensitivity and specificity of various staging investigations in comparison to staging laparoscopy and operative findings. Methods: 40 patients with periampullary tumors deemed resectable on preoperative investigations were evaluated prospectively. Demographic details, preoperative imaging details (Ultrasound abdomen (USG), CECT, MRI and EUS) were recorded. Staging laparoscopy was done for all patients who was followed by Whipples operation if the tumor was found to be resectable. The results of preoperative imaging were compared with staging laparoscopy and operative findings. Results: Obstructive jaundice was the most common presenting symptom (84\%). 17 patients (44\%) underwent ERCP with endoscopic biliary drainage and stenting. Staging laparoscopy could pickup 2 patients with liver metastasis thus avoiding unnecessary laparotomy in $5.8 \%$ of patients. On exploration 3 patients had unresectable tumor due to infiltration at the root of mesentery. 35 (84\%) patients underwent Whipple's procedure. The mean operative time was $260.8+50.3$ minutes and the mean operative blood loss was $1068.0+606.1 \mathrm{ml}$. CECT and MRI showed an accuracy of $85.2 \%$ and $82 \%$ in determining resectability. EUS was most accurate in tumor detection (100\%). Conclusion: CECT and MRI have almost similar sensitivity and specificity for detection of the tumor and vascular invasion. MRI does not seem to have any additional advantage over dual phase CECT. EUS however is a very sensitive and specific investigation for diagnosis and tumor detection. The lymph node and vascular invasion can also be better predicted on EUS performed by an experienced operator. The sensitivity, specificity and accuracy of EUS is better than both CECT and MRI. Staging laparoscopy has only a limited role in detecting small peritoneal and hepatic metastasis after a high quality CECT and EUS.

\section{PT-C9 \\ Endoscopic Ultrasound (EUS) Guided Fine Needle Aspiration (FNA) for Evaluation of Pancreatic Masses: Experience from Tropics}

\author{
S.S. Rana, D.K. Bhasin, R. Srinivisan, R. Gupta, K. Singh \\ Departments of Gastroenterology, Cytology and Surgery, \\ Postgraduate Institute of Medical Education and Research \\ (PGIMER), Chandigarh, India
}

Background: EUS FNA is an important diagnostic tool for evaluation of pancreatic masses. There is limited experience on its utility in tropical countries. Aim: To evaluate the accuracy of EUSFNA for diagnosis of pancreatic solid as well as cystic mass lesions. Methods: Over last 18 months, 40 patients with pancreatic mass lesions presumed to be resectable on $\mathrm{CT}$ abdomen were subjected to EUS FNA. The final diagnosis was obtained either by surgery or endoscopic retrograde Cholangiopancreatography (ERCP) or after clinical follow up. Results: Forty patients (24 males) underwent EUS and guided FNA for pancreatic solid (28) /cystic (12) mass 
lesions. The lesions were located in head (28), tail (7) and body (5) of pancreas. A mass lesion was observed on CT abdomen in 34/40 (85\%) patients. EUS picked up all the mass lesions and lesions missed on CT were less than $2 \mathrm{~cm}$ in diameter. The final diagnosis in patients with solid mass lesion was: adenocarcinoma (14), inflammatory mass (4), neuroendocrine tumor (4), pancreatic tuberculosis (3) and pancreatic metastasis from renal cell carcinoma (1). The final diagnosis in patients with cystic lesions was: Pseudocyst (10), simple cysts (1), mucinous cystadenoma (1), serous cystic tumor (1) and intraductal papillary mucinous tumor (1). EUS guided FNA yielded the correct diagnosis on first attempt in $37 / 40(92.5 \%)$ patients. Three patients required repeat FNA for achieving the correct diagnosis. Conclusion: EUS guided FNA of pancreatic mass lesions is an accurate tool for achieving diagnosis for both solid as well as cystic mass lesion especially for lesions less than $2 \mathrm{~cm}$ in diameter. Pancreatic tuberculosis is an important treatable cause that can be effectively diagnosed by EUS FNA in tropical countries and thus avoid unnecessary major pancreatic resection.

PT-C10

Utility of Endoscopic Ultrasound Fine Needle Aspirate (EUS FNA) for the Diagnosis of Pancreatic Cystic Lesions Not Associated with Pancreatitis

\section{S.S. Rana, D.K. Bhasin, K. Singh \\ Department of Gastroenterology, Post Graduate Institute of Medical Education and Research (PGIMER), \\ Chandigarh, India}

Aim: Pancreatic cystic lesions not associated with pancreatitis are a diagnostic dilemma and EUS has helped in better evaluation of these lesions. Methods: Retrospective analysis of all the patients with pancreatic cystic lesions not following an attack of pancreatitis referred to us for EUS was done. Results: Over last 20 months, 10 patients (6 M; age: 19-67 years) with pre EUS diagnosis based on cross sectional imaging of pseudocyst (5), cystic tumor (3) and IPMN (2) were evaluated. Of 5 patients with pseudocysts, EUS appearances were suggestive of pseudocyst (2), cystic tumor (2) and main duct IPMN (1). EUS FNA confirmed the diagnosis of cystic tumor in 2 patients (mucinous tumor (1) and pseudopapillary tumor (1) and main duct IPMN in 1 patient. However, 2 patients with appearances suggestive of pseudocyst on EUS had simple cyst (1) and mucinous tumor (1) diagnosis after FNA. Three patients with cystic tumor on cross sectional imaging had EUS appearances suggestive of serous tumor. EUS FNA revealed diagnosis of pseudocyst in these patients which was confirmed on subsequently performed ERCP and resolution after endotherapy. One patient with pre EUS diagnosis of IPMN had EUS features suggestive of pancreatitis with pseudocyst and EUS FNA also revealed diagnosis of pseudocyst. Another patient suspected of IPMN had EUS features of IPMN and EUS FNA confirmed it to be IPMN. Conclusion: EUS is an important investigational modality for diagnosis of pancreatic cystic lesions but EUS appearances alone are not helpful in differentiating various causes of cystic lesions and EUS FNA is required for diagnosing pancreatic cystic lesions accurately.

\section{PT-C11 \\ Application of Intraoperative Transluminal Core-biopsy for Mass in Periampullary Region}

K. Jiang, J. Chen, Z. Xu, Z. Qian, C. Dai, J. Wu, W. Gao,

F. Guo, J. Wei, Z. Lu, Y. Miao

Department of General Surgery, The First Affiliated

Hospital of Nanjing Medical University, China

Background: Preoperative pathological examination provides critical information for managing mass in periampullary region. However, preoperative endoscopic ultrasound (EUS)-guided biopsy is an expensive and non-routine procedure in developing countries, thus a reliable and cheap alternative is demanded. Aim: To evaluate the accuracy, safety and cost of a new intraoperative biopsy method for mass in periampullary region. Methods: Following Kocher's maneuver, paracentesis to the mass was made via duodenum transluminally, with a commercialized $16 \mathrm{G}$ core-biopsy needle. Three to 14 tissue specimens were obtained for frozen section examination. Depending on the pathological result, radical resection was performed or not. Malignancy status of the lesion was authenticated by postoperative pathological examination and/or long-term follow-ups. Results: From Jan. 2001 to Jun. 2010, intraoperative transluminal core-biopsys (TLCB) were applied in 335 cases in our surgical center. Intraoperative pathological examinations revealed 248 cases of adenocarcinoma, 12 atypical hyperplasia and 75 without evidence of malignancy. False positive rate, false negative rate, sensitivity and specificity of TLCB were $0 \%, 2.0 \%, 98.0 \%$ and $100 \%$, respectively. Complications were recognized in 2 cases $(2 / 335,0.6 \%)$, with one case of postoperative pancreatitis onset and the other of intraoperative intraluminal bleeding. No mortality related to TLCB was observed. The cost of TLCB per case is about $150 \mathrm{USD}$, which is $1 / 10$ compared with EUS-guided biopsy. Conclusion: TLCB is an effective, safe and cheap method for intraoperative diagnosis with mass in periampullary region, which can provide reliable evidence for surgical decision making.

\section{PT-C12 \\ The Role of Fat and Fibrosis in Pancreatic Texture and in the Incidence of Postoperative Pancreatic Fistula}

\section{T.V. Madhav, S. G. Uppin, N. Bheerappa, R.A. Sastry \\ Departments of Surgical Gastroenterology and Pathology, Nizam's Institute of Medical Sciences, Hyderabad, India}

Background: Although soft pancreas is traditionally associated with increased risk of pancreatic fistula, the histological features of soft and hard pancreas have not been clearly defined. Aim: To analyze the role of fat and fibrosis in pancreatic texture with particular reference to soft pancreas. In the incidence of postoperative pancreatic fistula. Methods: Between Jan 2007 and May 2009, 59 patients underwent Whipple's procedure of whom 13 (22\%) developed pancreatic fistula (Grade A: 6, Grade B: 4 and Grade C: 3 ). The pancre- 
atic texture was subjectively graded by the operating surgeon as soft (20) or hard (39). Histological sections from the pancreatic neck were graded (0-4) for intralobular fat, interlobular fat, total fat and fibrosis by standard methods. The amount of pancreatic fat and fibrosis was correlated with the softness of pancreas identified at surgery. The influence of fat and fibrosis on postoperative pancreatic fistula was analyzed. The influence of other factors like age, sex, diabetes, hypertension, alcoholism, smoking, BMI, preoperative stenting and chronic pancreatitis on the amount of fat and fibrosis were also studied. Results: Soft pancreas was identified as a significant risk factor (OR 10.8; CI 2.55-45.8; $\mathrm{p}=0.001$ ) for pancreatic fistula. Soft pancreas had more fat $(p=0.08)$ and less fibrosis $(p=0.09)$ when compared with hard pancreas. Negative correlation existed between fat and fibrosis $(\mathrm{R}=-0.148 ; \mathrm{p}=0.19)$. Intralobular fat $(\mathrm{p}=0.001)$, interlobular fat $(p=0.13)$, total fat $(p=0.03)$ and fibrosis $(p=0.017)$ were found to be significant in development of pancreatic fistula. Incidence of pancreatic fistula in patients with total fat score $>1$ was $40 \%$ whereas in patients with score $\leq 1$ it was $12.8 \%(p=0.02)$. No patient developed pancreatic fistula with fibrosis score $>1$. Fatty pancreas was more common with advanced age. Fibrosis was more common in smokers $(\mathrm{p}=0.09)$ and chronic pancreatitis $(\mathrm{p}=0.01)$. Undilated pancreatic duct $(<3 \mathrm{~mm})$ was more common in fatty pancreas $(p=0.04)$. Conclusion: Soft pancreas was associated with increased amount of fat and less amount of fibrosis. Pancreatic fat and fibrosis play a significant role in development of pancreatic fistula.

\section{PT-C13}

\section{Nonfuctional Neuroendocrine Tumors of the Pancreas: Clinical Presentation in 6 Patients}

\section{S.D. Shenvi, R. Gupta, G. Singh, R. Singh \\ Department of General Surgery, Post Graduate Institute of Medical Education and Research (PGIMER), Chandigarh, India}

Background: Pancreatic endocrine tumours (PNETs) are uncommon with an incidence of $<1$ per 100000 person-years in population studies. Non-functioning tumours comprise a substantial proportion of all PETs and have been reported to comprise $25-100 \%$ of all PNETs. Although these lesions are relatively benign, they may on occasion exhibit a far more malignant pattern than there functional counterpart. Methods: Case records of 6 patients of non-functioning PNET were retrospectively reviewed. Duration of study was 2001-2009. Results: Most common complaint was pain abdomen. Preop CECT shows characteristic presence of hypervacularity in 2 patients. 3 patients had liver metastasis at presentation. Preoperative diagnosis could be established in 2 patients with image guided FNAC. All the patients underwent surgical excision (Whipple's procedure). All the tumors were of well differentiated histology. Immunohistochemical studies were positive for chromogranin A in 4 patients and synaptophysin in one patient. Surrounding organ infiltration was seen in 3 patients. Peripancreatic lymph nodes were positive in 5 patients. 2 patients received adjuvant therapy. Among six patients 4 are alive and are on regular follow up. Conclusion: PNET differs from pancreatic adenocarcinoma by younger age of presentation, their immunohistochemical pattern and a better prognosis after surgi- cal resection. Aggressive resection of the primary tumor as well as metastatic lesions with adjuvant therapy may improve survival.

\section{PT-C14 \\ Laparoscopic Management of Cystic Neoplasm of Pancreas}

S.N. Kurhade, M. S. Peethambaran, M. Joshi, M. Gupta, N. Mohan, A. Ramesh

Department of Surgical Gastroentrology, Meenakshi Mission Hospital, Madurai, India

Background: Laparoscopic pancreatic resections for cystic neoplasms of pancreas are limited to small retrospective case series as these neoplasms are rare. We critically analyzed the indications for and outcomes after laparoscopic resection of pancreatic cystic neoplasm a tertiary referral centre in South India over 10 year period. Aim: To analyze retrospectively the outcomes of patients operated with laparoscopic pancreatic resection for cystic neoplasm of pancreas. Methods: Retrospective chart analysis of 2000 to 2010. Results: Total of 17 patients was admitted with cystic neoplasm confined to pancreas. 12 females and 5 were males. Age group varied from 17-56 years (mean 26.3 years). 13 patients had serous cystadenoma, 4 cases had mucinous cystadenoma. All underwent laparoscopic procedures. Enucleation was done in 2 patients, distal pancreatectomy with splenectomy was done in 6 patients, 6 patients underwent distal pancreatectomy with spleen preservation, and 3 cases underwent median pancreatectomy. The average operating time was $110-160 \mathrm{~min}$ (mean: $130 \mathrm{~min}$ ). None of the patients required blood transfusion. There is no conversion in any case, however in one patient after median pancreatectomy reconstruction was done by open method. The average size of tumor varied from $5-20 \mathrm{~cm}$ (mean $8 \mathrm{~cm}$ ). There was no mortality in the present series. 3 patients had post op external pancreatic fistula, which was managed conservatively. Fistula closed spontaneously over a period of 3 weeks. The average fistula volume was about $100 \mathrm{ml} .2$ patients had post op intra abdominal abscess, which was percutaneously aspirated. The average hospital stay was about 6days (5-17 days). Conclusion: Cystic neoplasms of pancreas can be managed safely by laparoscopic techniques with accepted morbidity and mortality in properly selected patients.

\section{PT-C15 \\ EUS Performs Better Than CT in Anatomical Localisation of Insulinoma}

A.J. Joseph

Department of Gastroenterology, Christian Medical College Hospital,Vellore, India

Introduction: After biochemical confirmation of the presence of insulinoma, imaging studies are used for anatomical localisation. We analysed the pick-up rate of EUS and MDCT in the localisation of insulinomas. Methods: From 2005 to 2010, 17 patients with a bio- 
chemical profile consistent with a diagnosis of insulinoma underwent both MDCT and EUS. Results: CT was able to localise the lesionin 11 out of 17, while EUS was able to localise the lesion in all those identified on CT and in 4 extra cases (15 out of 17 totally). Lesions which were missed on CT but picked up on EUS were located in the head of the pancreas and were smaller in size (median diameter $10 \mathrm{~mm}$ vs $26 \mathrm{~mm}$ ). Conclusions: EUS performs better in localising insulinomas when the lesions are small and located in the head of the pancreas.

\section{PT-C16}

\section{A Case of Hyper Insulinemic Hypoglycaemia}

\section{Vimalraj, S. Jeswanth, R. Surendran \\ Department of Surgical Gastroenterology, Stanley Medical College, Hospital, Chennai, India}

Objective: Insulin Autoimmune Syndrome (IAS) is a rare disease characterized by hypoglycemia and autoantibodies to insulin without prior insulin administration. Here, we report a case of IAS associated with alpha lipoic acid (ALA). Case report: A 28-yearold Indian female suffered from repeated episodes of weakness, sweating, palpitations, hunger and tremor. The symptoms disappeared after the ingestion of food. She noted weight gain, and she was admitted with an episode of unconsciousness in the late postprandial state and blood glucose was found to be $33 \mathrm{mg} / \mathrm{dl}$ with serum insulin levels of $>1000 \mathrm{mIU} / \mathrm{ml}$ (normal range $2.6-24.9 \mathrm{mIU} / \mathrm{ml}$ ). Her c peptide levels were $12.81 \mathrm{ng} / \mathrm{ml}$ ( normal range $0.81-3.85 \mathrm{ng} / \mathrm{ml}$ ), She was investigated and her CT scan revealed small lesion in midileal loop which was hyper vascular, pancreatic parenchyma showed no lesions, Nuclear Tc99m octride scan showed no demonstrable abnormal tracer avid lesion noted in whole body. Since the symptoms were severe patient was taken up for surgery and intraoperative ultrasound, the small ileal diverticula was resected first and frozen section showed adenomyomatous lesion [later confirmed by histopathology], intraoperative ultrasound was done which showed no obvious lesions, but two suspicious areas were removed and sent for frozen section, it was reported as normal pancreatic tissue, thorough examination of all the intraabdominal organs did not reveal any other lesion. Post operatively patient needed continuous correction of hypoglycemia till complete oral intake was resumed. Insulin autoantibody levels were measured and found to be in high titres. Careful reelicitation of drug history revealed intake of alphalipoic acid for treatment of chickungunya fever till she developed symptoms of hypoglycaemia. Her glycmic status improved and 4 weeks after stoppage of the drug her serum insulin levels was $31.4 \mathrm{mU} / \mathrm{L}$ (normal range $1.7-31.00 \mathrm{mU} / \mathrm{L}$ ) and $\mathrm{C}$ peptide was $1.55 \mathrm{ng} / \mathrm{ml}$ (normal range $0.81-3.85 \mathrm{ng} / \mathrm{ml}$ ). At present she is completely relieved of the symptoms. Conclusion: We report a possible relevance of alpha lipoic acid contained in a health supplement in a case of insulin autoimmune syndrome. For the strong antioxidative effect, ALA has come to be used widely as a health supplement for dieting and antiaging in world wide. Given that ALA has come to be widely used as a health supplement, it will be important to be more careful to watch for the development of IAS connected with ALA.

\section{PT-C17}

\section{An Analysis of Solid Pseudopapillary Neoplasms of Pancreas: 10 Yrs Experience}

R. Kamalakannan, E. Selvakumar, V. Vimalraj, D. Jyotibasu, R. Sukumar, L. Anand L, C. Sukumar, R. Ravi, S. Jeswanth, P. Ravichandran, R. Surendran Department of GI Surgery, Stanley Medical College,
Chennai, India

Background/Aim: Solid pseudopapillary neoplasm (SPN) is a rare tumour with low malignant potential comprising $1 \%$ to $2 \%$ of all pancreatic tumours. Aim of this study is to analyze clinicopathologic characteristics and surgical outcomes of SPNs. Methods: Clinical data between 2001 and 2010 were retrospectively analyzed for SPN. Ten cases of solid pseudopapillary tumours were reported in our institute. Clinico pathological features, radiological findings, surgical intervention and follow up details were reviewed. Results: Ten patients were identified as SPN. Nine patients were female (90\%). Median age was 23 years. Predominant symptom was abdominal pain (70\%). Eight patients $(80 \%)$ presented with solid cystic component. Two patients had solid tumour. Preoperative diagnosis of possibility of SPNs was considered in 8 patients $(80 \%)$ on basis of clinical and radiological findings. Radiological features suggestive of malignancy were seen in two. One patient was diagnosed as SPN by FNAC. Eight patients $(80 \%)$ underwent complete resection (distal pancreatectomy -7 , central pancreatectomy -1 ). One patient underwent palliative resection. Another one was inoperable and received palliative chemotherapy. On follow up (6 months to 8 years), patients who underwent complete resection did not develop recurrence. Patients who underwent palliative chemotherapy and palliative resection are on regular follow up (14 months and 24 months respectively). Conclusion: Preoperative diagnosis of SPN is crucial in order to propose the proper management. Offering palliative resection gives better results and associated with long term survival even in advanced SPNs.

\section{PT-C18 \\ Periampullary Carcinoma of the Young: How Do They Fare?}

J.R. Reddy, R.K. Singh, A.K. Tank, B. Pottakkat, A. Prakash, A. Behari, A.K. Gupta, V.K. Kapoor, R. Saxena

Department of Surgical Gastroenterology, Sanjay Gandhi Postgraduate Institute of Medical Sciences, Lucknow, Uttar Pradesh, India

Background: Periampullary carcinoma is usually a disease of old age. In the young it is expected to be associated with genetic syndromes and behaves aggressively with poor results. Aim: To analyze differences in presentation and outcomes of periampullary carcinoma between young $(\leq 40$ years $)$ and old $(>40$ years $)$ patients. Methods: Retrospective analysis was done from a prospective database of 320 consecutive patients with periampullary carcinoma who underwent pancreaticoduodenectomy (1990-2007). Fisher's exact test, Mann Whitney U test, Kaplan Meier and multivariate analysis 
with Cox proportional hazards tests were appropriately used. Results: There were 62 patients with age d' 40 years and 258 patients with age $>40$ years. There was no difference in demographics (expect age), presentation and operative parameters (except duration of jaundice - longer in young, $\mathrm{p}=0.02)$. The proportion of patients undergoing pylorus preservation was similar in both groups $(50 \%$ vs $48 \%$ respectively). Major morbidity, postoperative stay and pathologic characteristics were similar. Survival analysis showed similar median/3-year survival in both groups ( $\%$ vs $\%, p=0.095)$. Multivariate analysis showed tumour differentiation and nodal status to be independent predictors of poor outcome in young and old age groups respectively. Though no investigations were done to test for genetic syndromes, no patient manifested with metachronous malignancies in upper/lower gastro-intestinal tract, on follow-up. Conclusion: Contrary to common belief periampullary carcinoma in young is similar in presentation and outcome to that in old age. In this study most were not associated with genetic syndromes though this aspect needs to be further studied.

\section{PT-C19}

\section{Branch Duct Intraductal Papillary-mucinous Neoplasia (BD-IPMN): 'Tightrope Walking' Between Surgery and Observation}

\author{
F. Giovinazzo, R. Salvia, G. Zamboni, P. Antonio, G. Malleo, \\ G. Butturini, G. Mascetta, A. Esposito, C. Bassi
}

Pancreatic Surgical Unit, G.B. Rossi Hospital of 'G.B.Rossi', University of Verona, Verona, Italy

Aim: To detect cancer progression risk groups in patients undergone to surgery affected by BD-IPMNs. Methods: 52 specimens from patients with a pathological diagnosis of BD-IPMN and undergone to surgery between 1993 and 2009 was reviewed. Magnetic Resonance Cholangiopancreatography (MRCP) and pathological findings were correlated with grades of dysplasia (low, moderate, severe and invasive carcinoma) and histological types (gastric, intestinal, pancreatobiliary and oncocitic). Results: Malignancy was statistically significant in Gastric type with a diameter more than $3 \mathrm{~cm}$ $(p<0.05)$, while in Intestinal type there was no statistical correlation with the main cystic size. Dilated main pancreatic duct, mural nodule and thickness of the cystic wall at MRPC were significant in relation to cancer progression for both grade of dysplasia and histological subtypes in all the histological types $(p<0.05)$. Conclusion: The risk to develop a carcinoma is different in BD-IPMNs histological types. Gastric type seems to present an indolent cancer progression behavior when compared to intestinal type. Mural nodules, thickness of the cystic wall and increased diameter of the main pancreatic duct at MRCP remain signs of malignancy. In conclusion BD-IPMNs are families of lesions in which we are still clinically enable to recognize the biological behavior previously to the resection.

\section{PT-C20}

Factors Affecting Post Operative Complications After Standard Pancreaticoduodenectomy for Periampullary Carcinoma

\author{
U. Chakrapani, B. Singh, K. Ravindranath, \\ R. V. Raghavendra Rao \\ Global Hospitals, Hyderabad, Andhra Pradehs, India
}

Background: With the increasing availability of the Imaging modalities and early diagnosis of periampullary carcinoma the number of patients being operated with curative intent has increased. The number of surgeons getting trained in pancreatic surgery and many of them performing pancreaticoduodenecomy in peripheral centers has increased. This demands the study of factors affecting the post operative complications which will help surgeons in predicting the most common complications at the earliest. Aims: To study the patient, disease, treatment and tumor factors in predicting the most common post operative complications. Methods: Patients diagnosed to have periampullary carcinoma who underwent standard pancraticoduodenectomy during Jan'06 to Dec'09 were studied for post operative complications. Pre operative biliary stenting was done in patients with serum total bilirubin $\geq 9 \mathrm{mg} / \mathrm{dl}$, in patients with features of cholangitis and in patients in whom surgery was delayed due to comorbid factors. The patient, disease, treatment and tumor factors were recorded and post operative complications were studied. Results: Among 47 patients with periampullary carcinoma who underwent standard pancreaticoduodenectomy with curative intent, 4 $(8.5 \%)$ patients died in the immediate post operative period. One or more complications were seen in $23(48.9 \%)$ of patients and the most complications were wound infection $(38.3 \%)$, delayed gastric emptying $(25.5 \%)$ and pancreatic leak $(23.4 \%)$. The significant factors responsible for delayed gastric emptying were diabetes mellitus, history of weight loss, serum albumin $\leq 3 \mathrm{mg} / \mathrm{dl}$ and pancreatic leak. The significant factors affecting pancreatic leak were diabetes mellitus and albumin level $\leq 3 \mathrm{mg} / \mathrm{dl}$. The significant factors responsible for wound infection were diabetes mellitus, total serum bilirubin $>10 \mathrm{mg} /$ $\mathrm{dl}$, preoperative stenting and duration of surgery $\geq 7$ hours. Conclusions: Applications of patient, disease, treatment and tumor factors helps in predicting post operative complications. Diabetes mellitus, Preoperative serum album level, preoperative biliary stenting and duration of surgery play a significant role in predicting immediate post operative complications.

\section{PT-C21 \\ Cystic Lesions of Pancreas Mimicking Cystic Neoplasms: Lessons Learnt}

\section{N. Saravanan, B. Singh, R. V. Raghavendra Rao \\ Department of Surgical Gastroenterology, Global Hospital, Hyderabad, India}

Background: Incidence of pancreatic lesions with cystic features has increased with advances in imaging and the improved outcomes of pancreatic surgery. Aims: To study the clinical, morphological and biochemical features in order to determine charac- 
teristics differentiating the varied pancreatic lesions with cystic changes. Methods: Pancreatic cystic lesions with suspicion of neoplasm between 2008 and 2010 at our institute were reviewed in a retrospective cohort. Results: Thirteen (52\%) of the 25 lesions were cystic neoplasms $(\mathrm{CN})$. Of the 12 others, 5 were pancreatitis related, 4 were ductal adenocarcinoma with cystic degeneration and one each of pancreatoblastoma, neuroendocrine and islet cell tumor. Among the CN's, 5 were benign, 6 were borderline (BL) and 2 were malignant (WHO classification). Benign and BL cysts were smaller (mean size $-8.7 \mathrm{~cm})$ compared to malignant ones $(20 \mathrm{~cm})$. Mean serum CA 19-9 was elevated in the malignant group ( 893 vs 7.2 in benign and BL group). Homogeneity and absent cyst wall enhancement characterised benign or BL lesions. Male-to-female (M:F) ratio in CN's was $0.18: 1$. Solid pseudopapillary tumor was seen in much younger females (mean age - 19 vs 61.4 in other CN's). The otherwise typically solid tumors, with cystic features were small $(4.9 \mathrm{~cm})$, had variable morphology and elevated serum CA 19-9(mean - 341). Mean age was $51(\mathrm{M}: \mathrm{F}=2: 1)$. Pancreatitis related cysts (suspected to be neoplastic when definite evidence of pancreatitis was lacking or CA 19-9 was elevated) were characteristically smaller $(2.1 \mathrm{~cm})$ with no septation or wall enhancement. Mean age in this group was 37 $(\mathrm{M}: \mathrm{F}=1.5: 1)$. Conclusions: Morphology including size, age and sex are useful clinical pointers. High serum CA 19-9 raises preoperative suspicion of malignancy in pancreatic cystic lesions.

\section{PT-C22}

\section{Serous Cystadenoma of the Pancreas: Experiences with 130 Resected Tumors at the MGH}

N.P. Valsangkar, S.P. Thayer, A.L. Warshaw, C. Fernándezdel Castillo

Division of General and Gastroenterologic Surgery, Massachusetts General Hospital, Harvard Medical School, Boston, USA

Background: Serous cystadenomas (SCA) are commonly diagnosed benign cystic tumors of the pancreas that are increasingly being identified in asymptomatic patients. Methods: A retrospective database of patients with SCA who were resected at the MGH was analyzed for patient demographic, tumor and outcome information. Results: Between 1990 and 2010; 130 patients were resected for SCA at the MGH. $66 \%$ were female and the mean age at diagnosis was 63 . Males were significantly older $(69$ yrs vs. $61 \mathrm{yrs}, \mathrm{P}<0.05)$. Only $40 \%$ of the patients were symptomatic; abdominal pain $(69 \%)$ was the most common symptom followed by abdominal fullness $(10 \%)$, and jaundice (3\%). $60 \%$ of the patients were incidentally discovered. EUS was performed in 33\% and CT alone in $43 \%$ of the patients. Overall, median tumor size was $44 \mathrm{~mm}$; with larger tumors pre-2000; ( $55 \mathrm{~mm}$ vs. $34 \mathrm{~mm}, \mathrm{P}<0.05)$. Older patients ( $>65 \mathrm{yrs}$.) also had larger tumors $(51 \mathrm{~mm}$ vs. $36 \mathrm{~mm}, \mathrm{P}<0.05)$. Tumors $<4 \mathrm{~cm}$ were less likely to be symptomatic than larger tumors ( $22 \%$ vs. $72 \%$, $\mathrm{P}<0.05$ ). 34 were proximal resections (Whipple procedures), 53 had distal resections ( 30 with splenectomy). 12 cases had a middle pancreatectomy. The median length of hospital stay was 6 days. $32 \%$ patients had at least one complication after surgery, of which 10 had pancreatic fistula, 8 had an abscess and 5 had both. There were no peri-operative deaths. 5 and 10-year survivals were $99 \%$ and $90 \%$ and none of the deaths were tumor related. Conclusions: SCA is a benign disease process commonly seen in older females. It is increasingly being diagnosed as an incidental tumor and appropriate surgical intervention can be done with low operative morbidity and mortality.

\section{PT-C23 \\ Pancreaticojejunostomy (PJ) Versus Pancreaticogastrostomy (PG) in Reconstruction After Whipple Operation for Malignancy}

M.M.S. Bedi, M.D. Gandhi, A. Venugopal, B. Venugopal, H. Ramesh

Lakeshore Hospital, Cochin, India

Background: Despite many previous studies, a large randomized trial comparing the two main anastomotic techniques after Whipple resection is lacking. Many observational studies have shown superiority of PG while randomized trials have shown no difference in outcome. Aim: Prospective randomized controlled trial of PJ vs PG after Whipple resection. Methods: Period of study 2004 to current. 284 patients were screened and 248 patients were randomized. Two patients were removed from the study owing to surgeon preference. 126 patients underwent PJ and 122 underwent PG. Patient characteristics were similar in both groups. In both, a duct-to-mucosa technique was used. The following parameters were analyzed: mortality, major complications, pancreatic fistula as defined by high drain amylase at days 1, 3, 5 and 10, intra-abdominal collections, postoperative bleeding, hospital stay, time to resumption of oral intake, and need for postoperative intervention (endoscopy, interventional radiology or surgery). Data was analyzed using SPSS v 11.0. Results: 3 patients died (2 PJ, $1 \mathrm{PG})$. Postoperative pancreatic fistula rates were higher following PJ than PG (see Table). Patients had fewer episodes of postoperative bleeding after PG, shorter hospital stay, and less postoperative interventions. Oral intake was resumed earlier following PJ. Conclusion: Postoperative pancreatic fistulas occur more commonly following pancreaticojejunostomy. Intra abdominal collections, hemorrhagic complications, and hospital stay were more in the PJ group, although mortality rates are similar. There is thus a trend towards superiority of PG over PJ in terms of pancreatic fistula rates and hospital stay.

\begin{tabular}{llcl}
\hline Parameter & PJ $(\mathrm{n}=126)$ & PG $(\mathrm{n}=122)$ & $\begin{array}{l}\mathrm{P} \\
\text { value }\end{array}$ \\
\hline High amylase in drain on Day 3 & 50 & 22 & 0.06 \\
High amylase in drain on Day 5 & 32 & 12 & 0.03 \\
High amylase in drain on Day 10 & 11 & 2 & 0.008 \\
Postop fluid collections & 16 & 12 & $\mathrm{NS}$ \\
Delayed gastric emptying & 31 & 33 & $\mathrm{NS}$ \\
Hemorrhage & 7 & 2 & 0.009 \\
Need for intervention & 18 & 10 & $\mathrm{NS}$ \\
Hospital stay (median) in days & 16.5 & 14 & 0.03 \\
Mortality & 3 & 1 & $\mathrm{NS}$ \\
\hline
\end{tabular}




\section{PT-C24}

\section{Rapid Ultrasound Guided Fine Needle Aspiration Cytology [USG FNAC] of Pancreatic Mass Lesions}

\author{
N. Rout, S. Samantaray, S.P. Singh, M.K. Mohapatra, \\ R.N. Mallik, P. Nayak, O. Agrawal, C. Meher
}

Departments of Gastroenterology, Oncopathology and GI Surgery, S.C.B. Medical College and Patholab and Beam

Diagnostics, Cuttack, India

Objective: Pancreatic mass lesions are not infrequently encountered in clinical gastroenterology practice. Investigations like CT, endoultrasonography, and oncomarker studies are beyond the reach of most people in developing countries. This study was conducted to evaluate the utility of the inexpensive USG FNAC for diagnosis of such lesions. Methods: The study material consists of ultrasonographically detected pancreatic mass lesions who were subjected to USG FNAC of pancreas. FNAC was performed with $22 \mathrm{G}$ needle [32mm to $89 \mathrm{~mm}$ long] and $10 \mathrm{ml}$ syringe. Ultrasonography was performed by Philips Envisor machine with mutifrequency $2-5 \mathrm{mHz}$ convex and $3-12 \mathrm{mHz}$ linear probes. The smears were air dried and stained with standard Leishman's stain and HE stain. Results: During the period from April 2009 to March 2010, USG FNAC was performed in 1085 cases; USG FNAC of pancreatic lesions was performed in 26 cases (2.4\%). Pancreatic adenocarcinoma was diagnosed in 16 cases $(61.5 \%)$ while acute pancreatitis was diagnosed in 6 cases [23\%]. Males were affected more often than females (sex ratio was 3:1). Maximum cases [61.5\%] were noted during the 4th and 5th decades. No complications were encountered during the procedure. Conclusion: Pancreatic carcinoma is the commonest cause of pancreatic mass lesions in coastal Orissa. Ultrasound Guided Fine Needle Aspiration Cytology [USG FNAC] of pancreatic lesions is safe and efficacious for rapid evaluation of pancreatic mass lesions.

\section{PT-C25 \\ Examining More Lymph Nodes Strengthens the Prognostic Value of Lymph Node Ratio}

\section{N.P. Valsangkar, J.S. Michaelson, D.M. Bush, C. Fernández- del Castillo, A.L. Warshaw, S.P. Thayer \\ Division of General and Gastroenterologic Surgery, Massachusetts General Hospital, Harvard Medical School, Boston, USA}

Background: The ratio of metastatic lymph nodes to examined lymph nodes (LNR) has a disputed role in predicting outcomes in patients with pancreatic cancer. In some studies, LNR has greater prognostic value than the raw number of metastatic lymph nodes (LN). Methods: A national population-based dataset of 10, 254 patients who underwent surgery for pancreatic cancer was reviewed. Tumor and patient characteristics were evaluated across three subgroups based on the numbers of lymph nodes examined at the time of surgery: < 5 (L), 5 - $13(\mathrm{M})$, and $>=13$ lymph nodes $(\mathrm{H})$. Univariate and multivariate survival analyses were done using Kaplan-Meier (KM) survival curves and Cox hazards model. Results: There were no significant differences between $\mathrm{L}, \mathrm{M}$ and $\mathrm{H}$ in their age, sex or race $(\mathrm{P}>0.05)$. The mean $(\mathrm{SD})$ tumor size $(\mathrm{mm})$ was higher in $\mathrm{L} ; 40$ (28.75) compared to $\mathrm{M} ; 35.39$ (18.8) and $\mathrm{H} ; 36.62$ (22.17), $\mathrm{P}<0.05$ and a decrease in the mean LNR from $\mathrm{L}$ to $\mathrm{H}$ (L: $0.38, \mathrm{M}: 0.26, \mathrm{H}$ : $0.19, \mathrm{P}<0.05)$ was observed. In each sub-group; the age at diagnosis ( $>65 \mathrm{yrs}$ ), absolute numbers of positive LNs and high LN ratio $(>0.2)$ were associated with a worse survival. On multivariate analysis, a Cox model showed that LNR $>0.2$ had a hazard ratio $>1$ in each subgroup: HR $( \pm 95 \%$ CI), Median Survival (Mo.); L: 1.52 ( \pm 0.20$), 19$; M: 2.95 ( \pm 0.55$), 17$; H: $3.25( \pm 0.76), 14$. Conclusions: Our analysis suggests that LNR $>0.2$ has substantial prognostic value regardless of the number of nodes examined. However, the excess hazard exerted by a LNR $>0.2$ increases with the number of examined lymph nodes even though there are proportionally fewer positive lymph nodes found. This suggests that LNR must be taken in context of the number of LNs examined.

\section{PT-C26 \\ Intraductal Papillary Mucinous Neoplasia: A 3 Year Audit}

\author{
G. Singh, M. Wani, B. Kundil, H. Ramesh \\ Department of GI Surgery, Lakeshore Hospital, Cochin, \\ India
}

Background: Intraductal papillary mucinous neoplasia (IPMN) has been accepted as a separate entity. Many cases which were initially being managed as chronic pancreatitis are now being recognized to be IPMN. Aim: To study the presentation, patient characteristics and risk factors for carcinoma in patients with IPMN. Method: All the patients diagnosed to have IPMN from June 2007 to July 2010 were included in study. Retrospective analysis of prospectively collected data was performed. Results: 13 patients were diagnosed to have IPMN during the study period. Data was analyzed retrospectively. Mean age was 49.8 years (27-66). 4 patients were being managed as chronic pancreatitis for last few years. One patient had side branch disease while the remaining had main duct IPMN. Mean pancreatic duct diameter was $11.6 \mathrm{~mm}(6-21) .5$ patients presented with carcinoma. 8 patients presented with disease in head region. 7 patients underwent Whipple's pancreaticoduodenectomy while total pancreaticoduodenectomy was done in 1 patient. 1 patient died 1 month postoperatively from infected chylous ascites and septicemia, while rest are on follow up till now with a mean follow up of 11.61 months (1-36 months). One patient with IPMN carcinoma developed bilobed liver secondaries within 3 months of surgery. Analysis of risk factors for carcinoma was done by univariate analysis but none of the entered variables were found to be significant risk factors for carcinoma. Conclusion: IPMN is recently being recognized more commonly and may be confused as chronic panrceatitis. While it was regarded a more indolent disease as compared to adenocarcinoma, outcomes depend on histology. IPMN carcinomas may present with a rapid downhill course and metastatic disease. 


\section{Surgery}

\section{S1 \\ Organ Preserving Approach to Cystic Neoplasm of Pancreas}

\section{K. Prathapan}

Department of GI Surgery, Medical College, Calicut, India

Background: Traditionally cystic neoplasm of the head of pancreas are treated by pancreato duodenectomy and that of body and tail by distal pancreatectomy with splenectomy, with the risk of increased morbidity and mortality. Aim: To assess the safety and usefulness of organ preserving surgery for cystic neoplasm of pancreas. Methods: All patients with cystic neoplasm of pancreas were assessed for organ preserving resection. Only those with definite benign lesion based on imaging and tumor marker assay were considered for organ preserving surgery. Patients with cystic lesion in the head and neck region of the pancreas underwent duodenum preserving resection of head and uncinate process. Those with lesion in the body of pancreas underwent median pancreatectomy and those towards the tail underwent limited resection of the distal body and tail with spleen preservation. Results: Among the 20 patients attended our department between Jan2007 and July 2010, 10 patients underwent organ preserving surgery. 4 patients underwent duodenum preserving pancreatic head resection, 2 median pancreatectomy and 4 spleen preserving resection of distal pancreas. None of the patients developed recurrent disease on follow up. Conclusion: Organ preserving surgery should be offered in all patients with benign cystic neoplasm of pancreas. The risk of recurrent disease is negligible in properly selected patients.

\section{S2 \\ Prospective Comparative Study of Complications of Pylorus Preserving Pancreato-duodenectomy for Chronic Calcific Pancreatitis and Pancreatic Carcinoma Based on International Study Group Definitions}

\section{V.A. Iyoob, R.S. Sindhu, R. Rajan, B. Natesh}

Medical College Hospital, Thiruvananthapuram, India

Background: Adoption of international definitions for major complications after pancreatoduodenectomy provided a baseline for comparison and objective assessment. Our aim was to prospectively analyze complications following Pylorus Preserving Pancreatoduodenectomy (ppPD) in Chronic Calcific Pancreatitis (CCP) Vs Pancreatic Carcinoma (PAC). Methods: All patients who underwent ppPD from Jan 2008 to Sept 2010 in our unit were selected prospectively for the study. PpPD done by similar method, reconstructions standardized and a uniform postoperative protocol adopted.
Patient data recorded in detail. Major morbidities graded as per the international study group definitions. Results: Of the total 65 patients selected, 50 were with PAC and 15 with CCP. M:F ratio was 28:37. Mean age $56.5 \mathrm{yrs}$ (PAC) and 48yrs (CCP). Operating time, transfusion requirements, hospital stay and overall complications (26\% PAC Vs $26.6 \%$ CCP) were comparable. 6(9.2\%) patients died post op (all in PAC group). 6(12\%) patients developed pancreatic fistula (PF) in PAC group and none in CCP group (p value 0.4$) .10(20 \%)$ patients of PAC group and 2(13\%) of CCP group developed Delayed Gastric Emptying (DGE). In the subsets of patients with DGE, 80\% in PAC and $100 \%$ patients in CCP group were having associated complications. Hemorrhage 3(6\%), bile leak 2(4\%) all in PAC group, prolonged drain in 1 and wound infections in 2 patients each were other complications. Conclusion: Higher mortality in PAC group may be due to advanced age and co-morbidities. PF is rare in CCP following ppPD. Primary DGE is rare after ppPD in both groups. Most DGE occur following a secondary event (secondary DGE).

\section{S3 \\ Randomized Clinical Trial Comparing Dunking and Duct-to-Mucosa Techniques of Pancreaticojejunostomy Following Pancreaticoduodenectomy: An Interim Analysis}

V. Mangla, S. Pal, N.R. Dash, P. Sahni, T.K. Chattopadhyay

Department of GI Surgery, All India Institute of Medical Sciences, New Delhi, India

Background: Pancreaticojejunostomy (PJ) leak is a serious complication following pancreaticoduodenectomy (PD). Different techniques have been used for PJ anastomosis but none has been shown to be superior. Aim: To compare outcome between 'dunking' and 'duct-to-mucosa' techniques of PJ. Methods: Between January 2009 and March 2010, 40 patients undergoing a pylorus preserving or a classical PD were randomized to either 'dunking' or 'duct-tomucosa' technique of PJ. Patients with MPD $\leq 2 \mathrm{~mm}$ were excluded. The primary end point was postoperative pancreatic fistula (PF) rate as defined by the International Study Group on Pancreatic Fistula (ISGPF). The secondary end points included PF grade, postoperative stay and incidence of delayed gastric emptying, reoperation, postoperative haemorrhage and mortality. Results: There were 19 patients in the 'dunking' group and 21 in the 'duct-to-mucosa' group. The two groups were comparable with respect to demography, preoperative laboratory indices, pancreatic texture and diameter of the pancreatic duct. There was no operative mortality. The PF rate for the entire cohort was $15 \%(6 / 40)$ and the clinically significant PF rate (ISGPF Grade B or C) was $10 \%$. Four $(21 \%)$ and two $(9.5 \%)$ patients in the 'dunking' and 'duct-to-mucosa' groups, respectively, developed PF. There was no difference in the PF rate $(\mathrm{p}=0.31)$ or other complications between the two groups. The postoperative hospital stay was significantly longer in the dunking group $(\mathrm{p}=0.03)$ probably because of a trend towards higher incidence of delayed gastric emptying $(p=0.06)$. Conclusion: As of now, we have found no difference in $\mathrm{PF}$ rate between the two groups. We are continuing the study to achieve the required sample size. (CTRI/2010/091/00531) 


\section{S4 \\ Pancreaticoduodenectomy in a Non- specialised Unit: Our Experience}

\author{
S. Rath, A. Basu, D.P. Patra, S. Vatsal \\ Department of General Surgery, Institute of Postgraduate \\ Medical Education and Research (IPGMER), and Seth \\ Sukhlal Karnani Memorial Hospital, Kolkata, West Bengal, \\ India
}

Introduction: In this study our aim was to access the morbidity, mortality and the quality of life associated with pancreaticoduodenectomy performed in a non-specialised unit of government hospital. Methods: A total of 44 patients who underwent pancreaticoduodenectomy for periampullary cancers in the department of general surgery, IPGMER between 2004 and 2008 were evaluated. Preoperative data were collected in terms of patient symptomatology, clinical presentation and associated comorbidities. Pre-operative staging was done by radiological work up including USG, CECT whole abdomen, ERCP, MRCP etc. All the patients with resectable tumors underwent classical Whipple's or pylorus preserving pancreaticoduodenectomy. Perioperative parameters regarding operating time, amount of blood loss, size and extent of the tumor, size of the pancreatic duct were noted. Post operatively all patients were shifted to general surgical wards. Complications were identified on serial clinical examination, routine laboratory investigations, drain fluid amylase content, ultrasonography and ct scan of abdomen in selected cases. Complications were addressed mostly by conservative means and operative management was considered where needed. All patients who get discharged were followed up initially at 3 month intervals for one year, biannually for next two years and annually thereafter. Long term complications were evaluated by routine laboratory investigations and serial USG whole abdomen. Long term quality of life was assessed in terms of symptom free survival, cost of the procedure and its impact on the family. Results: A total of 44 patients with operable periampullary carcinoma were evaluated under this study. Out of them $20(45 \%)$ patients had carcinoma head of pancreas, $10(23 \%)$ patients had distal cholangiocarcinoma, 10 (23\%) patients had ampullary tumor and rest $4(9 \%)$ patients had duodenal carcinoma. $40(91 \%)$ patients underwent classical pancreaticoduodenectomy, and pylorus preserving pancreaticoduodenectomy was performed in rest 4 cases. Postoperatively all patients were put on general surgical wards. Enteral feeding through jejunostomy tube was started from 3rd postoperative day. 14 (31.8\%) patients developed pancreatic fistula of which 10 patients responded to conservative management with octreotide. 3 patients required relaparotomy in which drainage was done in 2 cases, repair of site of leakage was done in 1 case, and total pancreatectomy in the rest 1 case. Biliary fistula was noted in 5 cases out of which 2 had combined pancreatic and biliary fistula. $2(4 \%)$ patients had post operative hemorrhage and died in the immediate postoperative period. 4 (9\%) patients had significant upper gi hemorrhage. Other significant complications included delayed gastric emptying in $17(38 \%)$ cases and wound infection in $17(38 \%)$ cases. Intraabdominal abscesses complicating pancreatic fistula occurred in 13 (29\%) cases. Among medical complications postoperative MI occurred in 3 patients, renal failure in 4 patients and pneumonia in 3 cases. A total of 13 (29.5\%) patients died following the operation and of its complications. When year wise data were compared mortality rates were $14 \%$ in 2008 while it was $30 \%$ in $2007,33 \%$ in $2006,37 \%$ in 2005 and $50 \%$ in 2004 . Out of the 31 patients who were discharged $20(64 \%)$ patients were alive by 1 year, $6(19 \%)$ patients by 3 years. The overall mean survival was 15 months. The average cost during hospital stay was Rs. 32000 ranging from Rs. 18,000 to Rs. 86,000. Conclusion: Pancreaticodu odenectomy is a major surgical procedure now has become the treatment of choice for Periampullary carcinomas. With advancement of surgical techniques, postoperative care and better understanding of the disease the complication rate has dramatically reduced. Reports suggest that operative outcome is better and should be performed in high volume centers only. With changing trends and experience of the surgeons along with better anticipation and manage.

\section{S5 \\ Annular Pancreas: A Rare Cause of Duodenal Obstruction in Adult}

\author{
S. Rath, A. Basu, D.P. Patra
}

Department of General Surgery, Institute of Postgraduate Medical Education and Research,and Seth Sukhlal Karnani Memorial Hospital, Kolkata, West Bengal, India

Introduction: Annular pancreas is an uncommon congenital anomaly which usually presents itself in infancy and newborn. Rarely can it present in late adult life with wide range of clinical severities thereby making its diagnosis difficult. Case Report: A 61 year old female presented with 3 months history of postcibal distension of abdomen and vomiting. On examination there was dilated stomach with visible peristalsis in epigastrium. Upper G.I endoscopy revealed dilated stomach, 1st and 2nd part of duodenum with narrowing of D2-D3 junction. CECT abdomen excluded any mural and luminal pathology. On exploratory laparotomy there was annular pancreas encircling whole circumference of 2 nd part of duodenum. Roux-en-y duodenojejunostomy was done. The patient made an uneventful recovery. She remained well suffering no ill effects on follow-up. Conclusion: Annular pancreas was one of the rare causes of duodenal obstruction in adults. Pre-operative diagnosis is often difficult. CT scan can illustrate the pancreatic tissue encircling the duodenum. ERCP and MRCP are useful in outlining the annular pancreatic duct. Surgery still remains necessary to confirm the diagnosis. Duodenoduodenostomy and duodenojejunostomy are the procedures of choice. 


\section{S6}

\section{Preoperative CT Scan Can Predict Incidence and Severity of Pancreatic Fistula After Pancreaticoduodenectomy}

H. Tranchart, S. Gaujoux, V. Rebours, M.P. Vullierme, S. Dokmak, P. Levy, A. Couvelard, J. Belghiti, A. Sauvanet

Departments of HBP Surgery, Gastroenterology, Radiology and Pathology, Hôpital Beaujon, University Paris VII, France

Aim: Fatty pancreas and increased body mass index (BMI) are risk factors of pancreatic fistula $(\mathrm{PF})$ after pancreaticoduodenectomy (PD), however the influence of fat distribution is still unknown. This study aimed was to assess the value of preoperative CT scan to predict incidence and severity of PF after PD. Methods: From 2006 to 2007, 103 patients (M/F 0.56, mean age 58 years) underwent PD with pancreaticogastrostomy. Visceral fat area (VFA), subcutaneous fat area (SFA), global fat area (GFA), hepatic, splenic and pancreatic density ( $\mathrm{Dh}$, Ds and $\mathrm{Dp}$ ) were measured retrospectively on preoperative CT scans at the umbilicus level using a free software. On the pancreatic margin, fibrosis and fatty infiltration were scored by a pathologist blinded to postoperative course and patient information. Results: PD was mainly performed for adenocarcinoma (48\%), IPMN (20\%) and endocrine tumor (11\%). Overall morbidity was $46 \%$ and PF occurred in $37 \%$ (grades A, B and C: $47 \%, 39 \%$ and $13 \%$ respectively). In univariate analysis, male gender $(p=0.02)$, $\mathrm{BMI}>25 \mathrm{~kg} / \mathrm{m} 2(\mathrm{p}=0.02)$, abdominal perimeter $>900 \mathrm{~mm}(\mathrm{p}=0.023)$, VFA $>8400 \mathrm{~mm} 2(p<0.0001)$, GFA $>24000 \mathrm{~mm} 2 \quad(p<0.012), V F A /$ GFA $>1 \quad(p=0.027)$ and $\mathrm{Dh} / \mathrm{Ds}<1$ (suggesting liver steatosis) were associated with a greater risk of grade $\mathrm{B}+\mathrm{C} \mathrm{PF}$. In multivariate analysis only $\mathrm{Dh} / \mathrm{Ds}<1 \quad(\mathrm{p}=0.04)$ and $\mathrm{VFA}>8400 \mathrm{~mm} 2(\mathrm{p}=0.04)$ were independent predictors of grade $\mathrm{B}+\mathrm{C} \mathrm{PF}$. VFA $>8400 \mathrm{~mm} 2$ was associated with fatty infiltration of the pancreas $(p=0.005)$. Conclusions: The increased visceral fat is associated with an increased risk of PF after PD. Thus, preoperative CT scan could help to predict the PF risk and to tailor preventive measures.

\section{S7}

\section{Cardiopulmonary Exercise Testing (CPX) in Assessment of Fitness for Pancreatico- duodenectomy}

\author{
M.A. Junejo, M.J. Parker, A.J. Sheen, A.K. Siriwardena \\ Departments of Anesthesiology and HPB Surgery, \\ Manchester Royal Infirmary, UK
}

Background: Pancreatic cancer is predominantly a disease of later life and many patients have considerable cardiovascular co-morbidity. Cardiopulmonary exercise testing (CPX) offers an objective and dynamic assessment of cardiopulmonary function. Aim: This study evaluates the role of CPX in risk assessment for pancreaticoduodenectomy. Methods: In a 3 year period from 1st September 2007 to 1st September 2010, 103 patients underwent pancreaticoduodenectomy and 4 patients had total pancreatectomy. Patients with co-mor- bidity and those aged over 65 were evaluated preoperatively with CPX. 55 (51\%) underwent CPX with a median age of 66 years (4579) $[\mathrm{p}=0.000]$. Outcome data were collected prospectively and analysed with CPET derived variables. Results: Anaerobic threshold (AT) did not predict cardiovascular complications, post-operative morbidity or outcome. Revised Cardiac Risk Index (RCRI), duration of surgery and intra-operative blood transfusion were associated with cardiopulmonary complications following surgery. In a logistic regression predictive model RCRI was the only predictor of cardiopulmonary complications. However, preoperative $\mathrm{VE} / \mathrm{VCO} 2$ and intra-operative blood transfusion were associated with post-operative mortality ( $p=0.031$ and 0.044 respectively). Overall 30 day postoperative mortality was $2.8 \%$. Conclusion: Contemporary peri-operative assessment must offer accurate risk assessment to address the high morbidity in a low mortality operation. Although these preliminary data suggest CPX to be a useful tool in identifying patients at risk following pancreaticoduodenectomy, it requires further evaluation of its role.

\section{S8}

\section{Lymphoepithelial Cyst - A Rare Non- malignant Cystic Lesion of Pancreas}

\section{U.P. Srinivasan, A.B. Duraisamy, G. Manoharan, S.M. Chandramohan}

\section{Department of Surgical Gastroenterology, Madras Medical College, Chennai, India}

Lymphoepithelial cyst of the pancreas (LEC) is a rare nonmalignant true cystic lesion, with only 88 cases being reported in the literature. We present a case of LEC, a 33 year old female with history of multiple abdominal surgeries, predisposing to incisional hernia, with an incidental finding of a cystic lesion in the pancreas. CECT revealed a cystic lesion of $5 \mathrm{~cm}$ diameter with mild wall enhancement in tail region of pancreas and a large incisional hernia. Serum Amylase, CEA, CA 19.9 was within the normal range. USGguidedaspirate was straw colored with elevated CA 19.9 and a normal amylase and CEA levels with Cytology negative for malignancy. Peroperatively there was extensive adhesions between bowel loops and sac wall and a thick walled cystic lesion of about $5 \mathrm{~cm}$ was found hanging from body of the pancreas. Distal pancreatectomy with splenectomy and Mesh repair for incisional hernia was done. Histopathology was consistent with LEC of the pancreas. Postoperative period was uneventful. This case is presented for its rarity and to emphasize that LEC should be considered in the differential diagnosis of cystic lesion of pancreas. 


\section{S9 \\ Adopting the Leed's Protocol with Colours in Pancreaticoduodenectomy - An Indian Experience}

C.S. Tampi, M. Hastak, D. Satpudke, R. Shah, P. Jagannath

Department of Histopathology, Lilavati Hospital and

Research Centre, Mumbai,India

Background: R0 surgery in Pancreaticoduodenectomy offers the only opportunity for cure. However, it is seen that recurrence rates after R0 and R1 resections are fairly similar,suggesting that positive margins are often under recognized and under reported. This calls into question the grossing and examination technique, and also which margin in the multiple margins around the pancreatic head are more important. While Transection margins are routinely examined and uncommonly positive in curative surgeries, it is the circumferential margins that define the difference between $\mathrm{R} 0$ and $\mathrm{R} 1$ surgeries. The Leeds protocol, with colouring of margins, now adopted in UK and Europe, currently offers the best method for examination. Aim: To assess the impact of Leeds protocol on the $\mathrm{R}$ status in Pancreaticoduodenectomies done at our centre. Method: 84 consecutive cases, of pancreaticoduodenectomy were analysed 42 before and 42 after adopting the Leeds protocol. Only cases of ampullary and pancreatic adenocarcinoma were studied and the differences in margin positivity before and after adopting the protocol are analysed. Result: On adopting the protocol, it is seen that the incidence of positive margins is more, the numbers of multiple positive margins is also increased, and medial margin positivity shows marked increase. Small ampullary tumors, hitherto thought to be completely resected, often showed periduodenal margin positivity. Conclusion: The Leeds Protocol lends itself to more accurate margin examination. Standardized protocols, once adopted, make patient care and comparision of data meaningful. Reporting margin positivity, gains importance, to identify patients most likely to benefit from adjuvant therapy, especially local Radiotherapy following resection, would depend on correct assessment of those who have minimal local residual disease.

\section{S10 \\ Traumatic Pancreatic Transection with External Pancreatic Fistula: It Is Possible to Salvage the Pancreas}

R.K. Singh, J.R. Reddy

Department of Surgical Gastroenterology, Sanjay Gandhi Postgraduate Institute of Medical Sciences, Lucknow, India

Background: Traumatic pancreatic transection of the main pancreatic duct invariably leads to a recalcitrant external pancreatic fistula. Current surgical options (distal pancreatectomy or a fistulo-jejunostomy) lead to loss or atrophy of distal pancreas. Aim: Distal pancreas preservation with a lateral pancreatico-jejunostomy. Methods: We managed 5 patients with external pancreatic fistula following blunt abdominal trauma. ERCP showed complete ductal transection at the neck or proximal body. At surgery the fistula tract was followed, the distal pancreatic duct was laid open longitudinally and a Roux n Y lateral (side-to side) pancreatico-jejunostomy done including the fistula site. Results: All 5 patients were young males (age 20-45 years). Associated injuries were liver laceration (1), hemoperitoneum (1), intrabdominal collections (2), ileal perforation (1). All presented with external pancreatic fistulae (through per-operative drains in 2 and percutaneous drainage of collections in 3). The mean duration from trauma to surgery for external pancreatic fistula was 193 (77-255) days. The mean operation time, blood transfusion and postoperative hospital stay were $4.4 \mathrm{hrs}(2-6), 1$ unit (0-3) and 7 days (6-10). There was no major postoperative morbidity or mortality, except minor wound infection. There was no leak, recurrence of fistula, collection or pseudocyst. At a mean followup of 16 months (6-52) CT scan showed preserved distal pancreas and no patient developed endocrine or exocrine insufficiency. Conclusion: External pancreatic fistula following traumatic complete pancreatic transection can be safely managed by a lateral pancreatico-jejunostomy with minimum morbidity, to preserve the distal pancreas and the pancreatic function.

\section{S11 \\ Delayed Haemorrhage After Pancreaticoduodenectomy - An Unusual Source \\ Y.C. Venugopal, R. Harish, M.K. Ekka, \\ S.S. Gopalakrishnaiah, M. Patil, K. Varghese \\ Department of Surgery, St John's Medical College, Bangalore, India}

Background: Haemorrhage after pancreaticoduodenectomy is a major life threatening complication accounting for $0.4-5 \%$ of its mortality. Aim: Presenting a case of delayed haemorrhage from an unusual source - a pseudoaneurysm of replaced right hepatic artery. Methods: A $55 \mathrm{yr}$ old man underwent pancreaticoduodenectomy for periampullary carcinoma of duodenal origin. On Postoperative day (POD) 7 patient developed vomiting, a pancreaticojejunostomy (PJ) leak (Type B) was detected, pigtailing of the collection was done. POD $15,500 \mathrm{ml}$ of fresh blood was detected through the right drain without any haemodynamic instability. Bleed from visceral arteries suspected, emergency angiogram was done. Results: Angiogram revealed a pseudoaneurysm of the replaced right hepatic artery. Stent graft was not possible due to tortuous nature of the vessel. Hence it was embolized with coils proximally and distally. Post procedure angiogram revealed a total occlusion of right hepatic artery. No further bleeding occurred, the PJ leak settled, patient recovered fully, discharged on POD 20. Conclusion: Pseudoaneurysms account for about $3 \%$ of delayed haemorrhage with a mortality of $14-58 \%$ after rupture. Vascular anomalies like replaced right hepatic artery are thoroughly delineated by angiography. Prompt angiography helps diagnose pseudoaneurysms and treat them with minimal morbidity and mortality and is the intervention of choice for massive delayed haemorrhage after PD. 


\section{S12 \\ Pancreaticogastrostomy Reconstruction of Pancreatic Stump After Pancreatico- duodenectomy for Periampullary Carcinoma}

\author{
V. Bansal, M.C. Misra, P.K. Garg, A. Krishna \\ Department of General Surgery, All India Institue of \\ Medical Sciences, New Delhi, India
}

Background: Management of the pancreatic stump after pancreaticoduodenectomy (PD) is still a matter of debate. Pancreaticojejunostomy $(\mathrm{PJ})$ is used commonly but is associated with a significant incidence of pancreatic leaks. Pancreaticogastrostomy (PG) is an alternative that has been reported to be safer. Methods: The study is a retrospective analysis of all patients undergoing PD for periampullary carcinoma in one surgical unit 25 years, with PG being the only drainage procedure for the pancreatic stump. Results: Among 320 patients, $170(53 \%)$ were male and $150(47 \%)$ were females. The median age of presentation was $51 \mathrm{yrs}$ (range 22-70yrs). The most common presenting symptom was that of jaundice and weight loss ( $84 \%$ ) with a mean duration of $3.3+2.4$ months. $14 \%$ of patients had cholangitis on admission. On investigation the average hemoglobin was 11.1 (range $6.3-14.7 \mathrm{gm} / \mathrm{dl}$ ), total billirubin 12.8 (range $0.6-$ $36.2 \mathrm{mg} / \mathrm{dl}$ ) and serum albumin levels of 3.8(range $2.2-5.3 \mathrm{gm} / \mathrm{dl}$ ). Of the 320 patients $140(44 \%)$ were stented preoperatively for either cholangitis (44/320) or high pre op billirubin levels $(>15 \mathrm{mg} / \mathrm{dl})$. Preoperative biopsy was available in $240(75 \%)$ patients with $70 \%$ showing adenocarcinoma and 5\% showing dysplasia. A staging laparoscopy was performed in all patients and 50(16\%) were found to have liver metastasis. Of the remaining 270 patients who underwent laparotomy, 45(17\%) were found to have unresectable disease due to SMV-PV axis infiltration. A total of 224 Whipples procedure were carried out along with one ampullectomy. A cholecystojejunostomy and gastrojejunostomy was done in 90 patients with 5 being done laparoscopically. Following Whipples operation, the pancreatic stump was managed with a double layered pancreatogastrostomy in all patients. The mean operative time $260.8+50.3$ minutes ranging from 180-390 minutes and the mean operative blood loss was $1068.0+$ $606.1 \mathrm{ml}$ ranging from $400-2600 \mathrm{ml} .16(5 \%)$ patients were reexplored in the immediate post operative period. The overall morbidity was seen in $60 \%$ patients the most common being wound infection. The incidence of minor pancreatic leak was $2.5 \%$ which was managed by conservative management. None of the patients developed a major pancreatic fistula. The overall mortality rate was $15 / 320(4.8 \%)$ with 12 deaths in early post op period (bleeding) and 3 delayed deaths due to septicemia (ventilator associated pneumonia). Conclusions: Pancreaticogastrostomy reconstruction following Whipple's procedure is a safe and effective alternative for pancreatic stump drainage.

\section{S13 \\ Middle Segment Pancreatectomy; An Additional Surgical Modality to the Pancreatic Surgeon}

\author{
Y. Wagh, G. Vikram, N.P. Chakravarthy, S. Paulvannan \\ Department of Surgical Gastroenterology, Kovai Medical \\ Center and Hospital, Coimbatore, Tamilnadu, India
}

Background: Pancreatic neck and proximal body lesions are usually resected by pancreatico-duodenectomy or distal pancreatectomy, often resulting in unnecessary loss of normal pancreas with potential exocrine and endocrine dysfunction. Middle Segment Pancreatectomy (MSP) is aimed at limited resection of the mid body to preserve functioning pancreatic tissue. Aim: Middle segment pancreatectomy is an additional tool in the management of pancreatic body pathology. Methods: MSP was undertaken for a 1) $9 \mathrm{~cm}$ large incidental mass in the neck and proximal body of pancreas and 2) Grade 3 Pancreatico-duodenal injury. The tumour was removed with $1 \mathrm{~cm}$ margin and proximal pancreas was closed with 3-0 prolene over a GIA stapler. The transected, necrotic pancreatic neck and proximal body was excised and the proximal pancreas was closed by 3-0 prolene sutures in the trauma patient. Peng's dunking Panceaticojejunostomy using a Roux loop of jejunum was done to reconstruct the distal pancreas in both the cases. Results: Case 1: Recovery without no leak/fistula and preserved exocrine-endocrine function at 1 year of follow up. Histology showed a completely excised solid pseudopapillary tumour. Case 2: Grade B pancreatic leak responded to conservative management with enteral nutrition, antibiotics and Octreotide. No exocrine-endocrine deficiency during the 2 month follow up. Conclusion: Middle segment Pancreatectomy is a safe, feasible and useful technique for selected patients with benign or low-grade malignant lesions and trauma involving the neck and proximal body of the pancreas. This additional surgical option in the pancreatic surgeon's armamentarium helps to preserve pancreatic exocrine and endocrine function and spleen.

\section{S14 \\ Re-operation Following Pancreaticoduodenectomy}

J.R. Reddy, R. Saxena, R.K. Singh, B. Pottakkat, A. Prakash, A. Behari, A.K. Gupta, V.K. Kapoor

Department of Surgical Gastroenterology, Sanjay Gandhi Postgraduate Institute of Medical Sciences, Lucknow, India

Background: Literature on re-operation following pancreaticoduodenectomy (PD) mainly describes early indications like post pancreaticoduodenectomy hemorrhage (PPH). Data on other indications are limited. Aim: To analyze the incidence and causes of reoperation during index hospitalization (early) and after index hospitalization (late) following PD. Methods: Retrospective analysis of 520 consecutive patients who underwent PD between May 1989 and September 2010. Results: 96 patients $(18.5 \%)$ were re-operated of which $72(76 \%)$ were early and $24(24 \%)$ were late re-operations. 
Among early re-operations median time to surgery was 8 days and indications were PPH (68\%), intra abdominal collection $(12.5 \%)$, hepaticojejunostomy (HJ) leak (2.8\%), duodenojejunostomy (DJ) leak $(5.6 \%)$, intestinal obstruction $(1.4 \%)$ and miscellaneous causes (9.7\%). Median hospital stay was 27 days and mortality was $36.1 \%$. Among late re-operations median time to surgery was 21 months. 13 patients were re-operated for complications of index surgery like incisional hernia $(n=4)$, pancreaticojejunostomy stricture $(n=2)$, adhesive sub acute intestinal obstruction (SAIO) $(n=2)$, HJ stricture $(n=1)$, DJ stricture $(n=1)$, enterocutaneous fistula $(n=1)$, gastroparesis $(n=1)$ and intra abdominal collection $(n=1) .8$ patients were reoperated for disease recurrence like peritoneal dissemination with SAIO $(n=4)$, liver metastasis $(n=3)$, and skin metastasis $(n=1) .3$ patients were re-operated for miscellaneous causes. Excluding patients re-operated for disease recurrence median survival was 49 months. Conclusion: Indications for late re-operations following PD are varied and complications related to primary surgery and radiotherapy can be successfully managed with good results.

\section{S15 \\ Magnification Assisted Pancreatico- jejunostomy (MAP-J) in Pancreaticoduodenectomy}

\section{D.G. Chhabra, K.G. Sutariya, R.G. Shah, P. Jagannath \\ Department of Surgical Oncology, Lilavati Hospital and Research Centre, Mumbai, India}

Background: Pancreatic fistula (PF) is the most significant cause of morbidity and mortality following pancreaticoduodenectomy. A precisely performed anastomosis is the key to reduce PF. Methods: A prospective database of 64 pancreatoduodenectomies performed in a single unit from 2008 through 2010 was analysed. An end to side duct-to-mucosal pancreaticojejunostomy (PJ) (without stent) was performed in all cases with fine polydiaxone interrupted sutures. PJ was completed with surgical loupes of $3.5 \mathrm{X}$ magnification in 32 patients (MAP-J group) and without in 32 patients (Control group). Drain fluid amylase was measured on postoperative day 3 and 7. Clinically relevant PJ leak (Grade B/C) was determined using the International Study Group of Pancreatic Surgery (ISGPF) classification. Pancreas related complications: collections, delayed gastric emptying (DGE) and haemorrhage were also analysed. Results: The patient characteristics i.e age, sex, pre-operative biliary drainage, type of resection, intra-operative texture of pancreas and mean pancreatic duct diameter were similar in both groups. Clinically relevant PJ leak occurred in $14 \%$ cases $(n=9)$. The leak rate was $3 \%(n=1$; grade B) in MAP-J group, compared to $25 \%(n=8 ; 4$ grade $B$ and 4 grade $C)$ in control group $(\mathrm{p}<0.001)$. Pancreas related complications included 7 pancreatic collections, 2 haemorrhages and 3 DGE in control group compared to single case of DGE in MAP-J group. Conclusions: Magnification assisted pancreaticojejunostomy (MAP-J) with increased visual acuity with surgical loupes significantly improves technical performance of PJ and reduces clinically relevant $\mathrm{PF}$ and related complications. We strongly advocate the routine use of surgical loupes with $3.5 \mathrm{X}$ magnification for a duct-tomucosa pancreaticojejunostomy.

\section{S16}

A New Type of Digestive Tract

Reconstruction After

Pancreaticoduodenectomy and Its

Preliminary Results

H. Wang

Institute of Hepatopancreatobiliary Surgery of PLA, Southwest Hospital, Third Military Medical University, China

Introduction: Pancreatic leakage is a severe complication after pancreaticoduodenectomy. Complicated pancreatic leakage may lead to sepsis and hemorrhage with a mortality rate of up to $40 \%$. The aim of this study was to design a new type of digestive tract reconstruction which may theoretically prevent the activation of pancreatic juice and the secondary lethal complications of pancreatic leakage. Methods: We designed new type of digestive tract reconstruction. Using two jejunal loops to do anastomosis with common bile duct and pancreatic duct respectively, then we do gastrojejunostomy after the confluence of bile and pancreatic juice. From Sept, 2010 to Oct, 2010 , four patients underwent a new type of digestive tract reconstruction after pancreaticoduodenectomy at Southwest Hospital, Third Military Medical University. Three patients are male, one patient is female. The average age is 60.5 years old. Data of patients was prospectively collected and analyzed. Results: The operating time was 330 minutes, blood loss was $387 \mathrm{ml}$. The amylase quantity of drainage went up to $1610 \mathrm{IU} / \mathrm{L}$ on POD 1, and came down to normal level on POD3. Digestive function thorough recovered on POD 3-5. There was no surgical mortality and severe complications. Conclusions: The new type of digestive tract reconstruction may prevent the activation of pancreatic juice and the secondary lethal complications of pancreatic leakage. However, we need multicenter clinical trial to demonstrate our conclusion.

\section{S17}

Middle Segment Pancreatectomy: Experience from a High-volume Center in China

Y. Miao, Z. Xu, Z. Qian, C. Dai, K. Jiang, J. Wu, W. Gao,

F. Guo, J. Chen, J. Wei, Z. Lu

Department of General Surgery, The First Affiliated

Hospital of Nanjing Medical University, China

Background: Middle segment pancreatectomy (MP) is an organ-preserving surgery for lesion located in neck or proximal body of pancreas, while its safety and effectiveness demand further evaluation. Aim: To review the experience of MPs in Department of General Surgery in The First Affiliated Hospital of Nanjing Medical University as a high-volume center for pancreatic surgery in China. Methods: From January 2004 to August 2010, 36 patients underwent MP in our center. Postoperative courses and long-term outcomes were recorded and analyzed. Results: Pathological results included 15 cystadenomas, 10 neuroendocrine tumors, 6 solid pseudopapillary 
tumors, 2 intraductal papillary mucinous neoplasms, 2 adenocarcinomas and a neurilemmoma. Mean tumor size was $3.7 \mathrm{~cm}$ and mean operation time was $253 \mathrm{~min}$ with a mean intraoperative blood loss of $416 \mathrm{~mL}$. There was no postoperative mortality or reoperation. Common postoperative complications were 'clinical' pancreatic fistula (5 patients, $13.9 \%$ ), intraabdominal fluid collection (3 patients, $8.3 \%$ ), hemorrhage ( 2 patients, $5.6 \%$ ), new-onset diabetes ( 2 patient, $5.6 \%)$. The complication rates were comparable to those following distal pancreatectomy (DP) during the same period in our center (all $\mathrm{p}>0.05$ ). Over a median 20 months of follow-up, all patients showed no local recurrence or metastasis. Deterioration of glucose metabolism was observed in one patient (2.8\%) and enzyme supplement dependence in 7 (19.4\%), which showed advantage over DP group (1/36 vs. $26 / 139, p=0.018$ and $7 / 36$ vs. $50 / 139, p=0.059$, respectively). Conclusion: MP preserves endocrine and exocrine function, and is a safe and reliable procedure for selected patients.

\section{S18 \\ Post-pancreaticoduodenectomy Chronic Pancreatitis}

\section{R. Saxena, J.R. Reddy}

Department of Surgical Gastroenterology, Sanjay Gandhi Postgraduate Institute of Medical Sciences, Lucknow, India

Background: Pancreaticoduodenectomy (PD) is a surgical operation with significant morbidity. Complications specific to PD, other than recurrences, that present on long follow up, are sparingly addressed. Aim: To describe stenosis of pancreaticojejunostomy (PJ) leading to obstructive chronic pancreatitis. Methods: 520 patients undergoing PD between May 1989-September 2010 for ampullary and other tumors at the author's Institution. Results: Two patients (19F, Choledochal Cyst with ampullary carcinoid, 1995; 52F, ampullary carcinoma, 2000) developed PJ stenosis following pylorus preserving pancreaticoduodenal resection (PPPDR). Two and a half years later both presented with intermittent upper abdominal pain of pancreatic origin. Investigations (US, CT, MRCP) revealed a dilated pancreatic duct in the residual pancreas and confirmed steatorrhea. An US-guided pancreatogram in case 1 showed a dilated pancreatic duct not communicating with the jejunum. After failed medical management both patients underwent surgical correction. The first patient underwent a conversion pancreatogastrostomy, and the second a revision PJ. The resected end of the pancreas showed only evidence of fibrosis and changes of chronic pancreatitis. 3 months later US showed a 3-mm duct. The first patient is asymptomatic for more than 12 years and the second for more than 7 years after reoperation. The second patient had a $6 \mathrm{~mm}$ pancreatic calculus and duct filled with sludge. Conclusion: Symptomatic PJ stenosis is rare following PD. Symptoms manifest more than 2 years after surgery and chronic pancreatitis that responds poorly to medical treatment is completely reversed with remedial surgery.

\section{S19 \\ Risk Factors for Delayed Gastric Emptying After Pancreaticoduodenectomy}

\author{
T.V. Madhav, N. Bheerappa, R.A. Sastry \\ Department of GI Surgery, Nizam's Institute of Medical \\ Sciences, Hyderabad, India
}

Background: Delayed gastric emptying (DGE) is the commonest complication after pancreaticoduodenectomy. Although DGE is known to be associated with other intra-abdominal complications, factors responsible for DGE in patients without intra-abdominal complications are poorly understood. Aim: The aim of the present study is to analyze factors causing delayed gastric emptying. Material and Methods: Between Jan 2007 and June 2009, 64 patients underwent Whipple's procedure. Delayed gastric emptying occurred in 29 of these patients (45.3\%) according to ISGPS criteria (Grade A-14; Grade B-8; Grade C-7). DGE without intra-abdominal complication was seen in 12 patients (18.7\%). The risk factors studied were Patient factors (age, sex, co-morbid factors, alcoholism, ASA physical status score, BMI, hemoglobin, total leukocyte count, serum creatinine, and serum albumin), Disease factors (liver function tests, cholangitis, preoperative pancreatitis, site of tumor, histopathology), Operative factors (preoperative biliary drainage, total operative time, blood loss ), Technical factors ( pylorus preserving or classical, pancreaticogastrostomy vs. pancreaticojejunostomy, antecolic vs. retrocolic gastrojejunostomy) and Postoperative factors (postoperative ventilatory support, time of initiating enteral feeds). Risk factors were analyzed for DGE and DGE without intraabdominal complications. Results: Significant risk factors on univariate analysis for DGE were: diabetes $(p=0.11)$, BMI $(p=0.05)$, preoperative biliary drainage $(\mathrm{p}=0.12)$ and intraabdominal complication $(\mathrm{p}=0.005)$. In a multivariate model adjusting for each of the univariate risk factors, significant factors independently predicting DGE were Intraabdominal complication (OR 6.5, CI 1.424-14.95, $\mathrm{p}=0.011$ ) and Diabetes (OR 3.5 , CI $0.926-27.04, p=0.06)$. On the other hand, risk factors for DGE without intraabdominal complications were age $(\mathrm{p}=0.13)$ and retrocolic duodenojejunostomy $(\mathrm{p}=0.09)$ on univariate analysis. The sole significant factor independently predicting DGE without intraabdominal complication was retrocolic duodenojejunostomy (OR 3.99, CI 1.037-42.664, p=0.04). Conclusion: Retrocolic duodenojejunostomy was an independent risk factor for DGE in patients without intrabdominal complication. 


\section{S20 \\ Central Pancreatectomy - A Technique for Resection of Centrally Placed Benign and Traumatic Pancreatic Lesions}

M. Madhavan, V. Vimalraj, P. Senthilkumar, E. Selvakumar,

R. Ravi, L. Anand, C. Sukumar, D. Jyothibasu, S. Jeswanth,

P. Ravichandran, R. Surendran

Stanley Medical College, Chennai, India

Background: Conventional pancreaticoduodenectomy or distal pancreatectomy with or without splenectomy for lesions involving neck and body of pancreas results in substantial loss of normal pancreatic parenchyma with an unnecessary risk of exocrine and endocrine insufficiency and splenic loss. Aim: To evaluate the technical feasibility, safety and procedural outcome of Central pancreatectomy in carefully selected patients. Methods: Between Jan 2000 and Mar 2010, totally 15 patients (M- 4, F - 11, Mean age 30.7Y) underwent Central Pancreatectomy were retrospectively analyzed. Indications were traumatic pancreatic neck transection in 4 patients, non functioning cystadenoma in 5 patients, bleeding pseudoaneurysm 2 patients, SPET in 1 patient and Insulinoma in 3 patients. Pancreatic exocrine function was evaluated by questionnaire method and endocrine function by blood glucose monitoring. Results: None of the patients developed pancreatic fistula or complications requiring surgical/ radiological intervention. Morbidity rate was $20.4 \%$. Mortality was nil. At a follow up period of 6 months to 10 years, none of the patient newly developed exocrine and endocrine insufficiency and all patients were alive without any evidence of disease recurrence. Conclusion: Central pancreatectomy is safe feasible conservative strategy for traumatic and benign non eneucleable pancreatic lesions involving neck and body of pancreas. This surgical strategy allows the disease to cure with preservation of pancreatic exocrine and endocrine function.

\section{S21 \\ Analysis of Outcomes After Pancreaticoduodenectomy in Pancreatic Specialty Group and Non-pancreatic Group \\ C. Dai, Z. Xu, Z. Qian, K. Jiang, J. Wu, W. Gao, F. Guo, J. Chen, J. Wei, Z. Lu, Y. Miao \\ Department of General Surgery, The First Affiliated Hospital of Nanjing Medical University, China}

Background: Pancreaticoduodenectomy (PD) is one of the most technique-demanding procedures in general surgery. Better outcomes were reported in high-volume centers. Aim: To compare short- and long-term outcomes after PD for periampullary cancer between pancreatic specialty group and non-pancreatic group in a tertiary teaching hospital in China. Methods: From Jan. 1986 to Aug. 2010, 790 patients underwent PD in our hospital with 610 cases in pancreatic group and 180 in non-pancreatic group. Data were collected prospectively and analyzed in retrospective way. Results: Characteristics of the two groups remain comparative in terms of age, gender, comor- bidity and pathology. Intraoperative data showed that patients treated in pancreatic group required less intraoperative blood transfusion compared to their controls $(706 \pm 538 \mathrm{~mL}$ vs. $776 \pm 244 \mathrm{~mL}, \mathrm{p}=0.087)$. Surgeons in specialty group perform more radical operations, indicated by less positive margin and more lymph nodes harvested $(7.0 \%$ vs. $11.7 \%, p=0.046$ and $10.3 \pm 2.3$ vs. $9.6 \pm 1.9, p=0.002)$. There were more postoperative pancreatic fistula, hemorrhage and intraabdominal infection in non-specialty group $(9.7 \%$ vs. $15.6 \%, \mathrm{p}=0.027 ; 8.5 \%$ vs. $13.3 \%, p=0.055 ; 7.5 \%$ vs. $13.9 \%, p=0.009$, respectively). Increased mortality was also observed in non-specialty group, but not reaching statistical difference $(1.1 \%$ vs. $2.8 \%, p=0.221)$. Follow-up studies revealed comparative overall survival in these two groups. Conclusion: It is our suggestion that patients who demand a PD be treated in a pancreatic specialty group.

\section{S22}

\section{Salvage Surgery for ISGPF Grade C Pancreatic Fistulae After Pancreaticoduodenectomy}

\section{S. Govil}

Department of GI Oncology, Bangalore Institute of Oncology, Bangalore, India

Background: Pancreatic fistula remains the most dangerous complication after pancreaticoduodenectomy. While some fistulae heal with adequate drainage and nutritional support, approximately a third to half of these fistulae persist, remain high-volume, and are associated with other complications. These ISGPF Grade C fistulae require reoperation but management of the pancreatic stump is controversial. The alternatives range between completion pancreatectomy, pancreaticogastrostomy, pancreatic duct occlusion and external drainage of pancreatic juice. Methods: A retrospective review of a personal series of 298 pancreaticoduodenectomies performed between January 2000 and October 2010 identified 12 patients (4\%) with Grade C pancreatic fistulae as defined by ISGPF. Results: All patients underwent end-to-side pancreaticojejunostomy on the same jejunal limb as the hepaticojejunostomy. Patients were re-explored between 12 and 33 days after primary operation (median 16 days). Six $(50 \%)$ patients were managed with drainage of intra-abdominal collections only. All six died from multi-organ failure; haemorrhage was the terminal event in 2 of these patients. Two patients underwent completion pancreatectomy for postoperative pancreatitis. One of these two patients survived. Four patients underwent dismantling of the pancreaticojejunostomy, stapled transection of the jejunal loop and dunking pancreaticogastrostomy. All four of these patients survived despite two of them having bled from the gastroduodenal stump. Conclusion: Dunking pancreaticogastrostomy may be a useful procedure to salvage pattients suffering from ISGPF Grade C pancreatic leaks after pancreaticojejunostomy. 


\section{S23}

\section{Post-Pancreaticoduodenectomy Hemorrhage in Periampullary Carcinoma: Results and Outcome According to the ISGPS Classification}

\section{R.K. Singh, J.R. Reddy, A. Tank, B. Pottakkat, A. Prakash,} A. Behari, A.K. Gupta, V.K. Kapoor, R. Saxena

Department of Surgical Gastroenterology, Sanjay Gandhi Post Graduate Institute of Medical Sciences, Lucknow, India

Background: Post-pancreaticoduodenectomy hemorrhage (PPH) is a serious complication that has been classified by ISGPS (International Study Group of Pancreatic Surgery) in 2007. However the prognostic utility of this unified classification is not clearly known. Aim: We report our experience with factors predicting PPH, mortality associated with PPH and outcome using the ISGPS classification. Methods: Retrospective analysis of a prospectively maintained database was done (from January 2003 to September 2010). Fishers test, Mann Whitney test and multivariate analysis (SPSS) were done for factors associated with PPH and post-PPH mortality. Results: Pancreaticoduodenectomy was done for 302 patients of periampullary carcinoma. PPH incidence was $17.2 \%$ (52 patients); early onset - 8 patients (15.3\%) \& late onset - 44 patients (84.6\%); Mild hemorrhage - 18 patients $(34.6 \%)$ \& severe hemorrhage - 34 patients $(65.3 \%)$. ISGPS PPH grade was A, B and C in 6 $(11.5 \%), 30(57.6 \%)$ and $16(30.7 \%)$ patients respectively. $\mathrm{PPH}$ was managed with conservative treatment, surgery alone, transvascular embolization or both in $26(50 \%), 16(30.7 \%), 3(5.7 \%)$ and $7(13.4 \%)$ patients respectively. The overall mortality after PPH was $13.4 \%$ (7 patients), all with ISGPS grade $\mathrm{C}$ hemorrhage. Univariate analysis showed that factors associated with occurrence of $\mathrm{PPH}$ are increased bilirubin $(p=0.02)$, overall postoperative major complications $(\mathrm{p}<0.001)$, pancreatico-jejunal anastomotic leak $(\mathrm{p}=0.01)$, intraabdominal abscess $(\mathrm{p}<0.001)$ and septicemia $(<0.001)$. However only intra-abdominal abscess $(\mathrm{p}=0.007)$ and overall postoperative major complications $(p=0.001)$ independently predicted PPH in multivariate analysis. Univariate analysis of factors predicting mortality after PPH revealed that old age $(\mathrm{p}=0.02)$, low preoperative haemoglobin $(p=0.03)$, septicaemia $(p=0.01)$ and ISGPS grade of PPH $(p<0.001)$ were significantly associated with mortality. Though multivariate analysis did not reveal any of these factors to independently predict mortality after PPH, all 7 deaths occurred in ISGPS grade C hemorrhage. Conclusions: Intra-abdominal abscess and overall postoperative major complications predict $\mathrm{PPH}$ in multivariate analysis. Morality following PPH is significantly associated ISGPS grade C hemorrhage. The ISGPS PPH classification is useful for triage and improved multimodality management in order to achieve a low mortality after $\mathrm{PPH}$.

\section{S24 \\ Post Pancreatectomy Hemorrhage: An Analysis of Management and Outcomes}

\author{
R.P. Solanki, T.V. Madhav, N. Bheerappa, R.A. Sastry \\ Department of GI Surgery, Nizam's Institute of Medical \\ Sciences, Hyderabad, India
}

Background: Post pancreatectomy hemorrhage (PPH) is a lifethreatening condition and a significant cause of mortality especially when associated with sepsis/pancreatic fistula. Aim: To analyse clinical course and outcomes of post pancreatectomy hemorrhage at our institute over past 4 years. Methods: From January 2007 till date, 114 patients who underwent pancreatic resections were included in a prospective database. 93 patients underwent pancreatoduodenectomy, 9 distal pancreatectomy and splenectomy and 12 underwent central pancreatectomy. Hemorrhage was defined according to timing, severity and location and graded (ISGPS 2007). Diagnosis, management and outcomes of these patients were analysed. Results: Prevelance of PPH was 12/114(10.52\%). Out of these 12 patients 10 were pancreaticoduodenectomies for malignancy, one for borderline malignancy (NET) and one was distal pancreatectomy and splenectomy for pancreatic body and tail tumor. Early hemorrhage $(<24$ hours) was seen in six and late in six. The hemorrhage was severe in nine (early 5 , late 4). It was mild in three (early 1 and late 2 ) and subsided spontaneously. It was extraluminal in nine; intraluminal in two and one patient had both. Endoscopy was performed in three patients. CT angiography and conventional angiography were done in two patients and demonstrated the site of bleed in one patient. Four patients had grade A, six had grade B and two had grade C hemorrhage. Surgery was performed in seven patients (five early and two late) with severe bleeding and hemodynamic instability. Angiography could not be done in these patients because of their instability. The site of bleeding was SMA branch in two, pancreatic stump in two, GDA aneurysm in one, gastroepiploic artery in two (one demonstrated by angiography), retroperitoneal vessels in one. Pancreatic fistula (PF) was present in five patients and one of them developed fistula after bleeding episode. Out of these, four patients $(80 \%)$ with PF had late bleeding. Three patients had intra-abdominal collections which required imageguided percutaneous drainage. PPH-related mortality occurred in one patient and was associated with pancreatic fistula. Conclusion: Majority of PPH are severe. Late bleeding is often associated with pancreatic fistula or intra-abdominal abscess. Surgery should be promptly considered in early severe bleeding. Acceptable outcomes can be achieved with an aggressive policy of managing patients with $\mathrm{PPH}$ and hemodynamic instability.

\section{S25 \\ 107 Pancreatic Resections: Factors Affecting Mortality and Morbidity}

K. Suman Bhushan, T.V. Madhav, N. Bheerappa, R.A. Sastry

Nizam's Institute of Medical Sciences, Hyderabad, India

Background: Mortality after pancreatic surgery has decreased dramatically though morbidity rates remain high. Studies analyzing 
risk factors related to specific and overall complications are scant. Prediction of morbidity pre-operatively has not been studied. Aim: To identify risk factors for morbidity after all pancreatic surgeries and after pancreaticoduodenectomy (PD). To identify risk factors for PF, PPH and DGE with or without intra abdominal complications after PD. To identify predictive value of Possum \& P-Possum scores in assessing mortality and morbidity after pancreatic surgery. Methods: Retrospective analysis of a prospectively maintained database of all patients who underwent major pancreatic resection for benign and malignant conditions in the Dept of SGE, NIMS Hyderabad from Feb 2007- Jun 2009 is presented. Emergency pancreatic surgeries, metastatic disease, extra pancreatic pseudo-cysts were excluded. Complications were graded according to the claviendindo grading system and were analyzed for risk factors which included patient, disease, operative and post-operative factors. Statistical analysis was performed using SPSS 17 software. Results: 107 pancreatic operations were done of which 64 were PD. Mortality after pancreatic surgery was $2.8 \%$. Complications occurred in $54.2 \%$. Major morbidity (Clavien GR $3>$ ) was seen in $26 / 107(24.2 \%)$ of patients. Significant risk factors for morbidity after pancreatic surgery were soft pancreas, SGPT $>73 \mathrm{U} / \mathrm{L}$ and absence of chronic pancreatitis. Soft pancreas (OR: 5.864, CI 1.11-30.95, $\mathrm{p}=0.37$ ) for pancreatic fistula, Intra abdominal Complications( OR: 6.5, CI 1.424-14.95, $\mathrm{p}=0.011$ )and Diabetes (OR: 3.5, CI 0.926-27.04, $\mathrm{p}=0.06$ ) for DGE, Liver disease( OR: 26.76, CI1.72-415.29,p=0.01) and PF grade B and C (OR:7.52, CI0.96-58.98, p=0.05) for PPH were significant risk factors after PD. The Observed to predicted mortality ratio was $0.42: 1$ and 1.5:1 using POSSUM \&P-POSSUM and that of morbidity is $0.72: 1$. Conclusion: Although mortality is low after major pancreatic surgery, morbidity rates remain high. Soft pancreas, age $>50 \mathrm{yrs}$, SGPT $>73 \mathrm{U} / \mathrm{L}$ and absence of chronic pancreatitis are independent factors predicting morbidity after pancreatic surgery. Soft pancreas is an independent risk factor for PF. Significant factors predicting hemorrhage are liver disease and GRADE B and C $\mathrm{PF}$. Intraabdominal complications $\mathrm{s}$ and DM are independent risk factors for DGE. POSSUM and P-POSSUM prediction of morbidity and mortality was poor.

\section{S26 \\ Comparison of Short Term Outcome Between Laparoscopic Pancreaticoduodenectomy and Open Pancreaticoduodenectomy}

\author{
P. Senthilnathan, V. Vaitheeswaran, P.S. Rajan, S. Moorthy, \\ S. Gobu, C. Palanivelu \\ Gem Hospital, Coimbatore, India
}

Background: Having published our results of laparoscopic Pancreaticoduodenectomy recently, we have compared its short term outcome with that of open Pancreaticoduodenectomy. Aim: To compare the short term outcome after laparoscopic Pancreaticoduodenectomy and open Pancreaticoduodenectomy. Methods: Retrospective analysis of data of all Laparoscopic and open Pancreaticoduodenectomies performed between Jan 2007 and Jun 2010 was performed. In both Laparoscopic and open groups the reconstruction was by a pancreaticojejunostomy. In later half of the series a Roux en Y reconstruction was used. Results: There were 34 patients in the laparoscopic group and 62 in open group. The mean operating time was significantly shorter in the open group than in laparoscopy group ( $244 \mathrm{~min}$ vs. $348 \mathrm{~min}$ ). Blood loss was significantly more in open group $(320 \mathrm{ml} v \mathrm{vs} .74 \mathrm{ml})$. The hospital stay was shorter in laparoscopy group (8.2 vs. 11.4 days). The overall postoperative morbidity was lesser in Laparoscopy group (25.6\% vs. $38.4 \%$ ). There was no mortality in both the groups. The difference in the average number of lymph nodes removed by either method was not statistically significant (19 in laparoscopy group and 21 in open group) and margin positivity rate was also similar. Conclusion: Laparoscopic Pancreaticoduodenectomy has lesser morbidity and is as radical as open Pancreaticoduodenectomy.

\section{S27 \\ Clinical Validation of Postoperative Pancreatic Fistula}

D.G. Chhabra, K.G. Sutariya, R.G. Shah, P. Jagannath

Department of Surgical Oncology, Lilavati Hospital and Research Centre, Mumbai, India

Objective: To validate the clinical significance of Pancreatic Fistula (PF) defined by ISGPF (International Study Group for Pancreatic Fistula) and Strasberg's classification of Pancreatic Anastomotic failure and its impact on management after pancreaticoduodenectomy (PD) in a HPB specialty surgical unit. Background: Both, the ISGPF and Strasberg et al have proposed a retrospective classification for postoperative PF based on clinical parameters; however both have not been rigorously validated. Methods: Between March 2006 and October 2010, 90 patients underwent PD with a duct-to-mucosal pancreaticojejunostomy with an abdominal drain. Drain fluid amylase (DFA) was measured on postoperative day (POD) 3 and 7 in all patients and on POD 10 in patients with elevated levels ( $>$ thrice serum value) on POD 7. PF was defined by ISGPF and Strasberg's criteria. The outcomes including pancreas related complications were analyzed. Results: POD 3 DFA diagnosed $31 \%$ patients with ISGPF-PF $(n=28)$; ) as against $15.5 \%$ $(n=14$; ) on POD 7 DFA. 14/28 patients showed normal DFA values within 4 days without any change in management and outcome. Strasberg's criteria identified 14 (15.5\%) patients with PF similar to latter group of ISGPF. These 14 patients labelled as ISGPF Grade B $(n=12)$ and $C(n=2)$ were better categorised by Strasberg's grades $(3$ Grade 1, 9 Grade 2, 1 Grade 3b, 1 Grade 4a). ISGPF Grade A especially if defined by POD 3 DFA $(n=14)$ had no clinical relevance and misleading. Conclusions: DFA estimation on POD 7 is clinically more relevant and avoids unnecessary diagnosis of PF by ISGPF (biochemical / transient / Grade A ) for patients with no change in clinical course. Our study validates that the Strasberg's grading system better than the ISGPF classification for management. 


\section{S28}

\section{Drain Amylase Value Alone on Day-3 in Diagnosis of Pancreatic Fistula: Is there No Relevance for the Drain Output Amount?}

\section{K. Prakash}

Dept of GI Surgery, P.V.S. Memorial Hospital, Cochin, Kerala, India

Background: ISGPF definition formulated in 2005 stipulates 3 times the upper normal value of drain amylase on Day 3 to diagnose pancreatic fistula. There are many patients with high drain amylases on day 3 with no clinical consequences. Aim: To analyse the incidence and grading of pancreatic fistula and grading by ISGPF definition and to assess whether a cut off of $50 \mathrm{ml}$ on Day 3 to high amylase value results in missing out clinically relevant pancreatic fistulae. Methods: Clinical data of 115 patient s between 2006 to August 2010 who underwent pancreaticoduodenectomy were analysed to find out Grade A, B, C fistulae. The pancreatic fistulae patients were subsequently subdivided into Group 1 with drain output $>50 \mathrm{ml}$ and Group 2 with drain output $<50 \mathrm{ml}$. The incidences various grades of pancreatic fistulae, complications, morbidity and hospital stay were analysed. Results: 53 patients $(46 \%)$ out of 115 had pancreatic fistulae, Grade A 48 patients, Grade B 5 patients and Grade C 0 patients. When the group A and B were subdivided; Group 1 had 24 Grade A fistulae, 5 Grade B and in Group2, 24 were Grade A and no Grade B fistulae. The mean drain amylase of Group 1 and 2 were 1796.1 (1305) and 2559.9 (3496) respectively $(\mathrm{p}=\mathrm{ns})$. The drain amounts were 130.8 (248.2) and 13.2 (11.5) in group 1 and 2 respectively $(\mathrm{p}=0.02)$. The complicating and morbidity were more in group 1 than group2 and the hospital stay were 14.3 (4.89) and 13.4 (4.26) respectively. Conclusion: By applying a cut off value of $50 \mathrm{ml}$ to the high amylase content on Day 3 clinically relevant (grade B and C) fistulae are not missed. A low output high amylase value drain on Day 3 may be safely ignored as it does not indicate more risk for complications or morbidity.

\section{S29 \\ Delayed Gastric Emptying Following Pancreatoduodenectomy - Does Detection of a Pancreatic Fistula Have a Predictive Role?}

\author{
V.A. Saraf, Binoj S.T., Sudhindran S., Sudheer O.V., P. Dhar \\ Department of GI Surgery, Amrita Institue of Medical \\ Sciences, Cochin, Kerala, India
}

Introduction: Mortality rates following pancreatoduodenectomy (PD) have decreased significantly over the past two decades, but the morbidity rates continue to be a cause for concern. The two commonest causes of significant post operative morbidity following $\mathrm{PD}$ are development of pancreatic fistulae (PF) and delayed gastric emptying (DGE). Aim: The aim of this study was to determine the association; if any between the development of DGE in patients with PF who had undergone a pancreatoduodenectomy. Methods: The study was conducted in a high volume, tertiary care centre with a $6 \%$ three month mortality rate for PD. Any drain output with an amylase content more than 3 times the upper limit of the normal serum amylase fluid level ( $>300 \mathrm{IU} / \mathrm{L})$ at postoperative day 3 or later was considered a PF. Delayed gastric emptying is defined as any nasogastric tube intubation lasting more than 3 postoperative days (POD) or the inability to tolerate a solid diet by POD 7. The records of a total of 51 consecutive patients were analysed retrospectively. Statistical analysis was carried out by comparing the level of association between these factors by the Spearman rho correlation. Results: 22 (43\%) patients had pancreatic fistula and 15 (29\%) patients had delayed gastric emptying. 11 patients with PF went on to develop DGE. Spearman's rho correlation was 0.37 which was significant ( $p$ value 0.029). Conclusion: Morbidity rates continue to be high in patients undergoing PD. It could be a worthwhile effort to continue naso-gastric drainage for longer periods in patients who develop pancreatic fistulae, as they are more likely to develop delayed gastric emptying.

\section{S30 \\ Does Intra-operative Bile Culture Pattern Affect the Final Outcome in Whipple's Procedure?}

N.A. Tiwari

Department of GI Surgery, Amrita Institue of Medical Sciences, Cochin, Kerala, India

Background: Preoperative biliary thought to have a deleterious effect on the post-operative recovery following Whipple's pancreatoduodenectomy (PD). Aim: We looked at the correlation between bile culture positivity and postoperative morbidity and mortality. Method: Out of all patients who underwent PD in our hospital in the past 3 years, 97 patients whose data was complete were analyzed retrospectively in terms of their intra-operative bile culture and sensitivity pattern and their risk of morbidity (hospital stay, wound infection and post-operative pancreatic leak) and mortality. Results: There was no difference in the mortality between the bile culture positive (group 1) (4/45) and the bile culture negative (group 2) (4/52). Rates of pancreatic leak and wound infection were similar between the two groups. The post-procedure in-hospital stay was also not significantly different between the two groups ( $\mathrm{P}$ value 0.591 for males and 0.426 for females, not significant). Conclusion: Bile culture positivity does not have a statistically significant effect on the overall outcome of patients undergoing Whipple's procedure. 
S31

\section{Failure of Angio Embolization in Post Pancreatic Surgery Delayed Bleed is a High Risk Factor for Mortality}

S. Mahesh, R. Anthony, M. Sharma, M.M.S. Bedi,

A. Venugopal, V. Lekha, B. Venugopal, H. Ramesh

Departments of GI Surgery and Interventional Radiology, Lakeshore Hospital, Cochin, India

Background: The study analyzed the presentation and management of hemorrhage after pancreatic surgery (pancreaticoduodenectomy, distal pancreatectomy, median pancreatectomy and lateral pancreaticojejunostomy). Role of surgery and embolization were determined in the study respectively. Methods: The data of 406 consecutive patients who underwent pancreatic surgery in our hospital over a 5 year period was analyzed. These cases include Whipple procedures $(n=172)$, Frey procedures $(n=172)$, distal pancreatectomy $(\mathrm{n}=51)$, and others $(\mathrm{n}=11)$. The data was analyzed to determine a) frequency of postoperative hemorrhage, b) demographics, and c) risk factors which correlated with mortality. Results: Hemorrhage occurred in $20(4.92 \%)$ patients either in early postoperative period (first 72 hours; $n=5,25 \%$ ) or after $72 \mathrm{hrs}$ (delayed bleeding; $\mathrm{n}=15$, $75 \%$ ). Bleeding occurred in 10 patients after a Whipple procedure, 8 after Frey procedure, and 1 each after median pancreatectomy and enucleation of insulinoma respectively. 11 (55\%) patients presented with GI bleed, 7 (35\%) patients presented as intra abdominal (drain site) bleed, and 2(10\%) presented with combined GI and intra abdominal bleed. 4 patients with early bleed were reoperated out of which 3 were operated on day 1 of surgery. And the fifth was managed conservatively. One patient in the early group died due to non surgical causes. In the delayed group, $5 / 15$ was managed conservatively and none died. 10 patients required interventions - 9 angiography, and surgery in 4 cases. None of the patients who had successful angiography died, whereas 3 out of 4 patients who required reoperation died. One patient died in the early group $1 / 5$ of postoperative renal failure (multiple comorbid illnesses), and 2 patients in the delayed group (2/15; one of bleeding, and the other due to sepsis). $9 / 15(60 \%)$ patients with delayed presentation had pancreatic fistula in the post operative period. Other significant risk factors were positive bile cultures, combined bile and pancreatic leak, and friable pancreas at operation. Conclusion: Postoperative pancreatic fistula (clinical/ biochemical) is the main risk factor for delayed postoperative hemorrhage. Angiographic embolisation was the procedure of choice for controlling delayed bleeding. Failure of angiography and requirement for surgical hemostasis were factors which indicated high mortality.
S32

Long-term Functional Results in Patients with Pancreatic/Periampullary Cancer Treated by Whipple Resection with Pancreaticogastrostomy

M. Wani, M.M.S. Bedi, V. Lekha, B. Venugopal, H. Ramesh

Lakeshore Hospital, Cochin, Kerala, India

Background: There are concerns that pancreas-stomach anastomosis may result in poor exocrine pancreatic function and nutritional disturbances. Aim: Examine the records of patients with greater than 10 year survival after Whipple procedure for pancreatic/periampullary carcinoma to determine functional results. Methods: There were 86 patients (male 49, female 37, age range 51-77, median 57.5). Site of origin of tumour was ampulla $(n=34)$, bile duct $(n=32)$, duodenum $(n=15)$, and pancreas $(n=5)$. All cases underwent reconstructionafterWhippleresection by duct-to-mucosapancreaticogastrostomy. Patients were asked to answer a questionnaire which requested information about preoperative and current endocrine and exocrine status. The following parameters were examined: (body weight at surgery, and at 10 years; fecal chymotrypsin in 29 and fecal elastase in 49 (8 patients had no fecal testing); Blood sugar and $\mathrm{C}$ peptide levels. Results: 36 of the 86 patients had diabetes mellitus prior to surgery (3 diet control, 25 on oral drugs, and 8 on insulin) and no patient had steatorrhea before surgery. 10 years after surgery, 51 patients were diabetic (4 diet control, 32 oral drugs, 15 insulin) and 4 patients had symptomatic steatorrhea. Weight gain occurred in 47 patients, and was maintained. In 22 patients, there was gradual loss of weight associated with fat intolerance, but no steatorrhea. This was correctable by pancreatic enzyme supplementation. 17 patients lost weight (3 to $17 \mathrm{~kg}$; median $8 \mathrm{~kg}$ ) which was not corrected by pancreatic enzyme supplementation. Fecal elastase levels ranged between 65 and 275 units (median 150). Quality of life scores were excellent to good in 67/86 patients, fair in 12, and poor in 7 patients. Conclusion: Over $2 / 3$ of patients developed deterioration in pancreatic exocrine and endocrine function, though quality of life was preserved. 


\section{S33}

\section{Laparoscopic Median Pancreatectomy - An Ideal Way to - Preserve Parenchymal Function for Mid Body Tumors}

C. Palanivelu, P. Senthilnathan, P.S. Rajan, S. Rajapandian, P. Praveenraj, V. Vaitheeswaran, A. Ramanujam, Manoj Kumar A.P

Gem Hospital, Coimbatore, India

Background: Pancreatic body lesions are difficult to treat, one for the late presentation and other being removal of major portion of parenchyma. Distal Pancreatectomy for benign tumors results in endocrine disturbances particularly development of Diabetes Mellitus. Median or central pancreatectomy is an option for centrally located tumors but is technically difficult to perform. We present our experience of Minimally Invasive approach to treat these tumors. Methods: Since 2001, we have performed 5 median pancreatectomy, 3 of them were for females and 2 males. The mean age is 36 years and the size of the lesion is $3 \mathrm{cms}$. Four of those were done for mucinous cystadenoma and one for serious cystadenoma. Malignant tumors, borderline resectable tumours and large lesions more than $3 \mathrm{~cm}$ were excluded. Technique: Patient is placed in supine with leg split position and surgeon operates by standing between the legs. Entire pancreas is exposed from head to tail region by opening the gastro colic omentum and mobilizing the transverse colon down. The inferior border of pancreas is defined and pancreas is mobilized posteriorly taking care to avoid injury to splenic vein and artery. Pancreatic branches supplying the mobilized pancreas are divided. Endo G1A stapler is used to divide the pancreas proximal to the tumour and reinforced with 30 PDS sutures. Distally, pancreas is divided using harmonic shears and the central portion with the tumour is removed. Distal pancreatic stump is anastomosed to a Roux limb jejunum in 2 layers. Results: The mean operating time is $240 \mathrm{mins}$ and blood loss is $160 \mathrm{ml}$. (range $30-320 \mathrm{ml}$ ). Mean ICU stay was 1 day and hospital stay was 4.5 days. There was no leak and all margins were negative. Follow up ranges from 8 years to 2 months and till now no recurrence is being reported. Conclusion: Laparoscopic Median pancreatectomy is an ideal procedure for selected small lesions situated in the body of pancreas which preserves both exo and endocrine function of pancreas.

\section{S34}

\section{Pancreatic Transection: Primary Repair, Case Reports}

\section{M.K. Khare}

Department of Surgery, J.L.N. Hospital and Research Centre, Bhilai, Chhatisgarh, India

Aim: We wish to report our recent experience of three cases where we have done primary repair. Methods: An 18 year old male patient presented to us with a history of being run over by a tractor. After basic investigations he was taken up for emergency exploratory laparotomy. On opening up the abdomen the duodenum was found to be transected at the junction of D1 \& D2. The pancreas was also found to be transected at the head region in the line of SMV. A primary repair of the duodenum was done over a controlled duodenal fistula with a T- Tube, one limb of which was placed beyond the pylorus into the stomach. The head of the pancreas was closed and a roux loop of the jejunum was anastamosed with the pancreatic duct over a $16 \mathrm{G} \mathrm{IV}$ cannula (end to side). This was a duct to mucosa anastamosis. A feeding jejunostomy was done. This patient was operated within 6 hours of presentation. Case 2: Another 32 year old male patient presented to us with a history of trauma to the abdomen by a bullock cart wooden $\log$ in upper abdomen when he was riding a bike at high speed. Patient's hemodynamic status was stable at the time of presentation and was not deteriorating further hence patient was investigated further with a high suspicion for pancreatic injury. On CT scan of the abdomen the pancreas was found to be transected at the level of midbody. Patient was taken up for exploratory laparotomy and an end to end ductal anastamosis was done over a $18 \mathrm{G}$ Epidural catheter which was used to stent the pancreatic duct. The parenchyma was repaired over the anastamosis. This patient was operated within 24 hours of presentation. Case 3: a 22 year male patient presented to us with a history of trauma to the abdomen by bike handlebar. The CT scan abdomen was suggestive of pancreatic transection at the body region. Patient was taken up for surgery and the pancreatic duct was stented with a $16 \mathrm{G}$ epidural catheter over which it the duct was anastomosed in an end to end fashion. The pancreatic parenchyma was repaired over the ductal repair. This patient was also operated within 24 hours of presentation. Results: All the 3 patients had uneventful postoperative period and were discharged within a period of 8 to 16 days. On discharge there was no exocrine or endocrine deficiency. Conclusion: Primary repair of pancreatic transection with end to end ductal anastamosis over a stent or pancreaticoenteric anastomosis can be performed safely in the acute setting. 
Videos

\section{V1 \\ Minimally Invasive Retroperitoneal Pancreatic Necrosectomy (MIRP) in Necrotising Pancreatitis - Our Technique}

\author{
A. Nagpal, H. Soni, S. Haribhakti \\ Haribhakti Surgical Hospital, Ahmedabad, India
}

Introduction: Severe acute pancreatitis with infected necrosis is one of the most morbid diseases. Surgical debridement is needed for majority of patients. With increasing technology and experience, it is now possible to do necrosectomy by minimally invasive techniques. Aim: To describe our experience with the use of retroperitoneoscopic method for the debridement of the necrosis. Methods: 5 patients were treated by MIRP for infected pancreatic necrosis. The mean duration of conservative management before surgical intervention was 38 days. Evidence of infection was the presence of gas in retroperitoneum ( 3 cases) and FNA positivity for infection (2 cases). Entire decision-making process was tailor made for each case individually as per the patient's history, disease condition, disease location and morphology on CT scan. Results: MIRP was attempted in 5 patients who had disease involving mainly the distal pancreas and the left side of the abdomen. No patient needed conversion to open surgery. However, two patients required open necrosectomy later on. Mean operating time was approximately 86 minutes with mean post-operative hospital stay of 42 days. Three patients developed controlled pancreatic fistula after RP necrosectomy, which healed conservatively on follow-up (at 32, 17 and 47 days). Mortality occurred in 1 patient due to malignant hyperpyrexia. Conclusion: MIRP is a feasible technique with acceptable results in carefully selected patients by experienced surgeons. Retroperitoneal approach may be selected in patients with left-sided pancreatic necrosis with predominantly semi-solid debris.

\section{V2 \\ Single Incision Laparoscopic Pancreatic Surgery - Feasibility \\ S. Gadiyaram, N. Shetty \\ Manipal Institute of Liver and Digestive Diseases, Bangalore, India}

Background: Feasiblity of single incision laparoscopic (SIL) surgery has been demonstrated with variety of abdominal procedures, viz cholecystectomy, appendectomy, splenectomy etc. We herein report two patients who underwent SIL procedures for pancreatic disorders. Methods: Data on patients who underwent SIL pancreatic surgery was reviewed from a prospective data base of patients undergoing SIL surgery at Department of Surgical Gastroenterology, Manipal Institute of Liver and Digestive Diseases, Bangalore. Clinical details, operative technique, postoperative course and follow-up were studied. Results: Case Report 1: A 46 year old gentleman, presented with pain abdomen and loose stools. Clinical exam and laboratory tests revealed no abnormality. His Serum Chromogranin A levels were elevated at $970 \mathrm{ng} / \mathrm{ml}$ and an endoscopic ultrasound guided fine needle aspiration of the lesion showed a neuro-endocrine tumor. Computerised tomography (CT) of the abdomen showed a well circumscribed tumor in the tail of pancreas with infiltration into the splenic hilum. He underwent a Single Incision Laparoscopic Distal Pancreatectomy and Splenectomy. Histopathology showed a well differentiated neuroendocrine carcinoma with negative margins and no nodal involvement. The post operative period was uneventful and he was discharged on the 6th post operative day. He presented with an intraabdominal collections two weeks later which was managed by percutaneous catheter drainage. Case Report 2: A 48 years old female patient presented with pain abdomen following an episode of acute pancreatitis (idiopathic). CT of the abdomen showed a pseudocyst in relation to the tail of pancreas. A SIL cystogastrostomy was performed. She was discharged from the hospital on third postoperative day. She was readmitted and managed conservatively for a self-limiting upper gastrointestinal bleed from the cystogastrostomy anastomosis site (on upper endoscopy). Conclusion: We demonstrated the feasibility of SILS approach for distal pancreatectomy and cystogastrostomy. Longer laparoscope, use of innovative stomach retraction technique, stapling device and endosuturing skills facilitated the procedures. Complications which occurred were unrelated to the SILS approach but to the nature of procedure and were managed appropriately. We need larger studies to study the usefulness of SILS approach in the surgical management of pancreatic disorders.

\section{V3 \\ 'Five Vessel Clearance' for Pancreatoduodenectomy}

\author{
D.G. Chhabra, S. Vaze, R. Shah, P. Jagannath \\ Department of Surgical Oncology, Lilavati Hospital and \\ Research Centre, Mumbai, India
}

Background: R1 resection for pancreatic cancer has been shown to be detrimental to survival. R1 resections are better defined by a standardized histopathological examination introduced by the Leeds Pathology Protocol (LEEPP). Posterior and medial margins are the most frequently reported positive resection margins especially in pancreatic adenocarcinomas and distal bile duct carcinomas. Anatomically these margins correspond to major vascular structures which form boundaries on medial and posterior sides. Aim: To define limits of postero-medial resection in pancreatoduodenectomy. Methods: We introduce a 'Five vessel clearance' concept that was developed to ensure an adequate clearance by exposing all vessels forming the medial and posterior boundaries of the pancreas and the uncinate process. The five vessels that need to be exposed for an adequate clearance are IVC and the left renal vein posteriorly, superior mesenteric vein (SMV) and portal vein medially and hepatic artery going on to celiac artery and superior mesenteric artery with aortic window posteromedially. Results: The uncinate process shows one of the three variations stopping short sometimes on the right border of SMV or extending under the SMV and sometimes under the border of superior mesenteric 
artery. A clear definition of boundaries of resection increases the rates of $\mathrm{R} 0$ resection. We could achieve $\mathrm{R} 0$ resections for 30 consecutive pancreatic head resections with this technique. Conclusion: The video demonstrates the 'Five vessel clearance' as our adopted method of ensuring an adequate clearance of uncinate and retropancreatic tissues which are at higher risk for microscopic margin positivity.

\section{V4}

\section{Laparoscopic Median Pancreatectomy}

M. S. Peethambaran, S.N. Kurhade, M. Joshi, M. Gupta, N. Mohan, A. Ramesh

Department of Surgery, Meenakshi Medical Mission Hospital, Madurai, India

Background: Median or middle pancreatectomy is a parenchyma sparing surgical technique to reduce or prevent post operative exocrine or endocrine deficiencies. Laparoscopically performed median pancreatectomy is a highly skilled procedure and different techniques are reported in literature. Hereby we describe our technique and experience. Methods: Three patients aged 45 and 48 year old females and 53 year old male got admitted with serous cystic neoplasm of body of pancreas. The preoperative workups of both the patients were benign. The sizes were about $8 \times 8 \mathrm{~cm}$ in females and $4 X 5 \mathrm{~cm}$ in the male patient. All patients underwent laparoscopic median pancreatectomy with complete excision of the mass. The proximal end was sutured with Vicryl 2-0 in two layers in first two patients and stapled with oversewn in the third. The reconstruction used was pancreatico-gastrostomy in two layers hand sewn. In two patients it was done laparoscopically and in one patient open pancreaticogastrostomy was done. Inner layer was $2-0$ Vicryl and outer 2-0 silk interrupted. The operating time was 140,170 and 130 minutes. The blood losses were less than $150 \mathrm{ml}$. No postoperative morbidity was noted. Oral diet was started on 5th POD. Hospital stays were 8, 9 and 7 days. Conclusion: Laparoscopic median pancreatectomy is a feasible procedure with safe patient outcomes. MP is an effective procedure for benign and low grade cystic neoplasm of pancreas with reduced post operative exocrine $\&$ endocrine deficiencies.

\section{V5}

\section{Laparoscopic Cystogastrostomy}

M.S. Peethambaran, S.N. Kurhade, M. Joshi, M. Gupta, N. Mohan, A. Ramesh

Department of Surgery, Meenakshi Medical Mission Hospital, Madurai, India

Background: Various minimally invasive techniques for drainage of the pancreatic pseudocyst has been described like radiologic imaging with percutaneous catheter drainage and endoscopic drainage. Surgical management is still considered the gold standard of pancreatic pseudocyst management. Open surgical drainage can be accomplished via cystogastrostomy, cystoenterostomy (direct drainage or via a Roux limb), or resection. However, these approaches are associated with substantial morbidity and mortality ( 25 and 5 percent, respectively). Drainage can also be accomplished laparoscopically, an approach first described in 1994. Laparoscopic cystgastrostomy can be performed via an anterior transgastric approach, which requires an anterior gastrotomy for access and cystgastrostomy creation through the posterior gastric wall or a posterior approach through the lesser sac (requiring only a single gastrotomy in continuity with the pseudocyst). Here we describe the technique of laparoscopic cystogastrostomy via anterior gastrostomy. Methods: 36 year old male patient presented with abdominal pain on and off for three months. On investigations he was found to have a large pseudocyst of $15 \times 13$ $\mathrm{cm}$ in the lesser sac posterior to the stomach. The patient underwent Laparoscopic cystogastrostomy. Anterior gastrotomy was done using harmonic scalpel, cyst opened, contents decompressed, disc of posterior stomach wall excised, Cystogastrostomy done with $2-0$ vicryl sutures and the gastrotomy closed with $2-0$ vicryl continuous sutures. Post operative period was uneventful. Patient was discharged on 4th postoperative day. Conclusion: Laparoscopic cystogastrostomy is safe and feasible procedure with comparable outcomes with open surgery and have all the benefits of minimally invasive surgery with less morbidity, less pain, reduced hospital stay and overall reduced cost.

\section{V6 \\ Pylorus-preserving Pancreaticoduodenectomy with Technique Modifications \\ Y. Miao, Z. Xu, Z. Qian, C. Dai, K. Jiang, J. Wu, W. Gao, F. Guo, J. Chen, J. Wei, Z. Lu \\ Department of General Surgery, The First Affiliated \\ Hospital of Nanjing Medical University, China}

Background: Pylorus-preserving pancreaticoduodenectomy (PPPD) is a technique-demanding procedure with high postoperative complication rates. Aim: To share our experience of PPPD in a highvolume center for pancreatic surgery in China. Methods: PPPD in ourcenterisperformedwithmodificationsin:1.Pancreaticojejunostomy (P-J): The anastomosis is typically performed in end-to-side invagination fashion. The posterior inner row of anastomosis is first placed with interrupted stitches, taking substantial bites of pancreatic parenchyma and capsule on pancreatic side and full thickness bites of the bowel wall on jejunal side. After the anterior inner row subsequently finished in the same fashion, cut edge of the pancreas is dunked into the jejunal lumen. Then, the posterior outer row and anterior outer row are performed with interrupted suture sequentially, taking bites of pancreatic parenchyma and capsule on pancreatic side and seromuscular bites on jejunal side. The outer layer approximates the jejunal serosa to the pancreatic capsule, allowing invagination of pancreatic stump into jejunum. 2. Duodenojejunostomy (D-J): An end-to-side D-J is performed in antecolic fashion as routine. The clamped duodenal incisional margin is first cauterized for hemostasis and sealing the wall into one layer. Then the inner layer of anastomosis with running suture is placed, taking bites of the single layer on duodenal side and full thickness bites of the bowel wall on jejunal side. The outer layer is performed with continuous Lembert sutures. Results/Conclusion: Our modifications confer advantage of tech- 
nical convenience, making anastomosis easier to perform. With increased technical precision, better outcomes are guaranteed.

\section{V7 \\ Laparoscopic Pancreaticoduodenectomy - How to Make It Safe and Sound Oncologically?}

\author{
P. Senthilnathan, V. Vaitheeswaran, P. S. Rajan, \\ S. Rajapandian, C. Palanivelu \\ Gem Hospital, Coimbatore, India
}

Background: We have recently published our results of Laparoscopic Pancreaticoduodenectomy. This video will demonstrate how a radical and safe procedure can be performed. Technique: This video will show the following steps: - Wide exposure of C-loop of the duodenum and pancreas; - Moblization and nodal clearance; Resection of pancreas; - Reconstruction- Pancreato jejunostomy, Hepatico jejunostomy and duodeno jejunostomy; -Specimen Extraction; There will be emphasis and technical tips on safety, radicality and use of appropriate energy source and reconstruction.

V8

\section{Laparoscopic Whipple's Operation: Video}

K. Prakash, N. P. Kamalesh, Rajegowda, I. S. Vipin, A. Sylesh, M. Jacob

P.V.S. Institute of Digestive Diseases, Kochi, Kerala, India

Aim: To demonstrate the feasibility and to demonstrate the steps of performing Whipple's operation using laparoscopic approach.

\section{V9 \\ Totally Laparoscopic Pancreaticoduodenectomy (video)}

\section{Varma, S. Philip, S.K. Mathai, C. Panackel \\ Medical Trust Hospital, Kochi, India}

In this video, we present the case of a 37 year old man who initially presented with common bile duct stones, for which endoscopic stone clearance was done. After a temporary symptom relief of 7 months, he came with obstructive jaundice. Evaluation with CT scan and endosonography showed a distal bile duct tumor. He underwent totally laparoscopic pancreaticoduodenectomy and reconstruction with duct to mucosa pancreatico-jejunostomy, choledochojejunostomy and stapled gastrojejunostomy. He had an uneventful post-operative recovery. Histopathologic examination of the resected specimen showed poorly differentiated periampullary carcinoma with one positive lymph node out of 14 harvested and negative resection margins.
The essential surgical steps and difficulties encountered during the procedure are highlighted in this video.

\section{V10 \\ Single Incision Cystogastrostomy - Technical Feasibility and Short Term Results}

\author{
C. Palanivelu, P. Senthilnathan, P.S. Rajan, S. Rajapandian, \\ P. Praveenraj, V. Vaitheeswaran, A. Ramanujam, \\ A.P. Manoj Kumar \\ Gem Hospital, Coimbatore, India
}

Background: Single incision laparoscopic surgery is now being employed for increasing number of procedures and is now envisioned as an alternative to NOTES. The perceived advantages are better cosmesis and possible reduced postoperative pain. Methods: We studied the feasibility and short term postoperative outcomes in 3 patients who had pseudocyst pancreas and underwent single incision laparoscopic cystogastrostomy. All cysts were located in the retrogastric region. The sizes of the cysts were 9,12 and $15 \mathrm{cms}$ with wall thickness of more than $5 \mathrm{~mm}$. Two of them were males and one female. One had biliary pancreatitis and 2 had alcoholic pancreatitis with pseudocyst. Technique: The patient was placed in supine with legs split position. A small incision was made in the umbilicus which was extended $0.5 \mathrm{~mm}$ cranially, flaps raised on either side and 3 trocars one $10 \mathrm{~mm}$ and two $5 \mathrm{~mm}$ were placed through this incision. Different sizes of trocars were used, pointing out in different directions to avoid cluttering of instruments. Conventional laparoscopic instruments were used for the procedure. Anterior gastrotomy was made in the most prominent part of the bulge. After placing everting stitches in the anterior wall of stomach, the exact position and content of the cyst is confirmed by aspiration. At the summit of the cyst, posterior wall of the stomach and the cyst wall opened, fluid sucked out and a small portion of stomach and cyst wall removed. Cystogastrostomy stoma is formed by approximately cyst and posterior wall of stomach using 20 PDS continuous sutures. Finally, anterior gastrotomy is closed. Umbilical wound is closed with 1 prolene. Results: The mean operating time is 145 mins. Though we had difficulty in getting the triangulation for endo suturing, we managed all intra corporeal suturing with trocars angulated away from each other. The mean hospital stay was 3 days and analgesic requirement was for 1 day. There were no complications in the form of collection, delayed gastric emptying or wound infection. Conclusion: Single incision laparoscopic cystogastrostomy is technically possible but operating time is slightly more. Immediate postoperative outcomes are comparable with laparoscopic surgery but require large series to assess the exact place of single incision approach for such procedures. 


\section{V11}

Laparoscopic Pancreaticogastrostomy After Whipples Pancreaticoduodenectomy

\section{C.J. Varghese, A.J. Kavalakkat \\ Westfort Hospital, Thrissur, India}

Objective: Laparoscopic Whipples Pancreaticoduodenectomy is considered to be technically difficult operation. A video of the Laparoscopic Whipple procedure with Pancreticogastrostomy done at our center is presented here. Methods/Results: This video is on Total Laparoscopic Whipples Pancreaticoduodenectomy performed in Nov 2008 for a patient with Periampullay carcinoma. Five ports were used. Mobilisation and reconstruction were done as in open Whipple's procedure. The stomach was transected at the Pylorus. Jejunum was transected close to DJ flexure. Neck of Pancreas and the uncinate process was divided using ultrasonic shears. Finally Common Bile Duct was transected. The reconstruction was done as Pancreaticogastrostomy (Duct to mucosa), Choledochojejunostomy and side to side Gastrojejunostomy. The Pancreatico gastrostomy was done in 4 layers in following order. Anterior outer Pancreas to Stomach with 00 silk continuous stich, Pancreatic duct to Gastric mucosa with 00 vicryl interrupted stitches (Anterior first and then posterior), posterior Pancreas to stomach with 00 silk continuous stich. The specimen was extracted through a $5 \mathrm{~cm}$ size ifra- umbilical incision. The surgery time was 480 mins. The blood loss was $250 \mathrm{ml}$. The post operative stay was 16 days. Conclusion: The entire surgery was done by Laparoscopic approach. The pancreatic anastomosis is most crucial in Whipple's surgery. Laparoscopic pancreaticogastrostomy can be performed with accuracy and precision as the suturing is from anterior to posterior unlike pancreatico jejunostomy.

V12

\section{Single Incision Laparoscopic Spleen Preserving Distal Pancreatectomy- Is it Feasible?}

C. Palanivelu, P. Senthilnathan, P.S. Rajan, S. Rajapandian, V. Vaitheeswaran, Saravanakumar, A. Vij

Gem Hospital, Coimbatore, India

Introduction: As innovation continues to move 21st century surgery forward, one of the emerging concepts is single-port or single-incision laparoscopic surgery. The fundamental ideais to have all of the laparoscopic working ports entering the abdominal wall through the same incision. The major drawback to such a surgical approach is that the concept of "triangulation" to which laparoscopic surgeons have grown accustomed in terms of both the instruments and scope is lacking. This, however, seems to be overshadowed by the increasing acceptability of in-line viewing, with the reemphasis on surgeons performing flexible endoscopy and on newer ideas such as natural orifice translumenal endoscopic surgery. Methods: This video describes the step by step approach of single incision mullti port technique using conventional laparoscopic instruments for distal pancreatectomy. Step1. Incision and flap rising' Step 2: Placement of ports;
Step 3: Opening of gastro colic omentum and exposure of pancreas with Suture retraction of stomach. Step 4: Mobilization of the pancreas, attempt to preserve the splenic artery and vein was made by clipligation of the pancreatic branches of the splenic vessels, then the transection of pancreaswas done using harmonic and stump closed with 2-0 PDS sutures; Step 5: Specimen extraction done inendobag through the same incision. Conclusion: Single port spleen preserving distal pancreatectomy is safe and viable in experienced hands and can be done with conventional instruments.

V13

\section{Single Incision Laparoscopic Lateral Pancreaticojejunostomy for Chronic Pancreatitis - A Short Case Series with Technique Description in a Tertiary Centre}

\author{
C. Palanivelu, P. Senthilnathan, A. Ramanujam, \\ A.P. Manoj Kumar, P. Praveenraj, \\ C. Chandra Maliteeswaran, P. Das Gupta \\ Gem Hospital, Coimbatore, India
}

Introduction: Laparoscopic single-site surgery for cholecystectomy and appendectomy are described in the literature. The benefits of these procedures compared with traditional laparoscopic approaches have yet to be determined. To date, no series of Single incision lateral pancreaticojejunostomy has been published or documented. This study isaimed to determine the safety and feasibility of SILS surgery for chronic pancreatitis. Methods: This study describes the technique and results of Single incision lateral pancreatico-jejunostomy in our series of 2 patients. Technique: Ø Incision and flap rising $\varnothing$ Placement of ports $\varnothing$ Opening of gastro colic omentum and exposure of pancreas with suture retraction of stomach $\varnothing$ Identification of the duct and laying opening of the duct, extraction of the stone, uncinate duct opening $\varnothing$ Creation of roux limb and pancreaticojejunal anastomosis. Results: The average duct diameter was $1.1 \mathrm{~cm}$. The average time taken was 210 minutes and the average blood loss was $60 \mathrm{ml}$. Both the cases were completed without any need for additional ports. The immediate ( 24 and $36 \mathrm{hrs}$.) post-operative pain score is comparable to our laparoscopic series. The scar score at 7 days is better in single incision surgery compared to laparoscopic group. Conclusion: In our experience, this technique is both feasibleand safe for selected patients. Although technical limitationsexist that will be improved upon, further studies are needed to compare single incision laparoscopic surgery with traditional laparoscopic technique. 
Abdo, E.E. AP-C34

Abdullaev, Y. AP-C23

Abraham, B. CP-C30

Aditya, A. AP-C28, M14

Agarwal, A., New Delhi CP-B4, PT-B3

Agarwal, Y. AP-C45

Aggarwal, G. PT-B2

Aggarwal, R. AP-C31

Agrawal, A.,

Chandigarh AP-C8, AP-C9

Agrawal, O. PT-C24

Agrawal, P.O. AP-C37

Aikot, S. AP-C32

Ajith Roni, D. AP-C47

Akbar, Md. AP-C28, M14

Akkim, S.K. AP-C47

Alagammai, P. M22

Ammini, A.C. M5

Anand, B. M10

Anand, L. AP-C27, PT-C17, S20

Anthony, R. S31

Antonio, P. PT-C19

Apte, M. PT-B8, M15

Arulselvan, V. AP-C42

Arunkumar, K. AP-C42

Arunkumar, M.L. CP-C17, M20, PT-C5

Augustine, J. CP-C22, CP-C28

Augustine, P. CP-C33

Aussilhou, B. PT-C4

Babu, B.I. AP-B6, AP-C2, AP-C36

Babu, Y.R. AP-C17

Bada, V.C. AP-C44

Balakrishnan, V. AP-C16, CP-C10, CP-C35

Balakumaran, L.K. CP-C24

Balantsova, E.L. M1

Balsarkar, D. M13

Banks, P.A. AP-C38

Bansal, V. PT-C8, S12

Bapat, M. AP-C12, CP-C9

Baranwal, S. M13

Bartoli, E. CP-C34

Basho, J. AP-C30

Bassi, C. PT-C19

Basu, A., Kolkata AP-C11, S4, S5

Basu, A., USA PT-B2

Basu, R. PT-B2
Bedi, M.M.S. CP-C29, CP-C30, CP-C31, CP-C32, PT-C23, $\mathrm{S} 31, \mathrm{~S} 32$

Behari, A. PT-C18, S14, S23

Bekolay, A. CP-B5, CP-B6

Belghiti, J. PT-C4, S6

Bhalla, A. AP-C22

Bhandari, S. AP-C12, CP-C9

Bhandari, V. AP-C45

Bharadwaj, M. M21

Bhardwaj, P. CP-C19

Bhasin, D.K. AP-C17, AP-C18, AP-C19, AP-C20, AP-C21, AP-C22, CP-C11, CP-C13, CP-C26, M7, M16, PT-C9, PT-C10

Bhaskar, S. CP-B3

Bhat, F. AP-C23

Bheerappa, N. CP-C12, M10, PT-C12, S19, S24, S25

Binoj, S.T. S29

Boggi, U. M12

Bollen, T.L. AP-C38

Booth, D.M. AP-B2, AP-B8

Bush, D.M. PT-C25

Butturini, G. PT-C19

Byelyayeva, N.V. CP-C2, CP-C3

Campion, E.R. M19

Cane, M.C. AP-B8

Chacko, A. CP-C22, CP-C28, M22

Chakrapani, U. PT-C20

Chakravarthy, N.P. S13

Chandail, V.S. AP-C19

Chandak, G.R. CP-B3

Chandra Maliteeswaran, C. V13

Chandramohan, S.M. S8

Chandrasekaran, P. AP-C18

Chari, S.T. AP-C10, PT-B2

Chattopadhyay, T.K. CP-C20, CP-C21, M5, S3

Chawla, A.S. AP-C45

Chejara, R. AP-C45

Chen, J. PT-B4, PT-C11, S17, S21, V6

Cherif, R. PT-C4

Chhabra, D.G. S15, S27, V3

Chidambaram, S. M9

Choubal, G. M5

Choudhuri, G. AP-C31, AP-C35

Chowdhury, S.D. CP-C28, M22
Chowdry, S.M. AP-C5, AP-C6

Chugh, R. PT-B9, PT-B12

Clemens, M. AP-C13, AP-C15

Coelho, A.M.M. AP-B5, AP-C34, M19

Copplestone, J. AP-C49

Couvelard, A. PT-C4, S6

Criddle, D.N. AP-B1, AP-B2, AP-B8

Cyriac, J. CP-C33

Daga, S.V. CP-C12

Dai, C. PT-B4, PT-C11, S17, S21, V6

D'Albuquerque, L.A.C. AP-B5, AP-C34, M19

Dare, A. AP-B7

Das Gupta, P. V13

Das, H.S. CP-C25, CP-C27

Daschakraborty, S. AP-C31

Dash, N.R. CP-C20, CP-C21, S3

Dawra, R. CP-B5, CP-B6, PT-B9

D’Cruz, S. AP-C29, M11

de Bortoli, N. M12

De, S.P. AP-C11

Del Chiaro, M. M12

Delcenserie, R. CP-C34

Deshpande, G. PT-C6

Devarbhavi, H. CP-C14

Dhar, P. AP-C39, AP-C40, S29

Dhar, S. AP-C4

Dhir, V. AP-C12, CP-C9

Dinesh, B.V. AP-C4

Dixit, P. AP-C7

Doctor, N.H. AP-C37

Dokmak, S. PT-C4, S6

Dudeja, V. PT-B9, PT-B12

Duraisamy, A.B. S8

Dutta, A.K. CP-C22

Dutta, U. AP-C5, AP-C6, AP-C7

Dwivedi, S. M17

Eddleston, J. AP-C2

Egawa, S. PT-C2

Ekka, M.K. S11

Elliott, V. AP-B2, AP-B8

Esposito, A. PT-C19

Evans, A. AP-C50

Faria, M.F. PT-B11
Fernández-del Castillo,

C. PT-C22, PT-C25

Fiala-Beer, E. PT-B8

Fine, D. M18

France, M.J. AP-C2

Fuks, D. CP-C34

Fukui, T. AP-B3, CP-B7

Gadiyaram, S. V2

Gadola, S. M18

Galeev, S. AP-C23

Gamanagatti, S. M5

Gandhi, M.D. AP-C41, PT-C23

Gandhi, V. M10

Gandhi, V.V. AP-C37

Gao, W. PT-C11, S17, S21, V6

Garg, P.K. AP-B4, CP-C18, CP-C19, CP-C20, CP-C21, M5, PT-C8, S12

Gasiorowska, A. PT-B1

Gaujoux, S. PT-C4, S6

Geetha, M. AP-C16

George, J., New Delhi CP-C20, CP-C21

George, J., UK AP-C49

George, T.J. AP-B4

Ghoshal, U.C. AP-C31, AP-C35

Giovinazzo, F. PT-C19

Girinadh, L. AP-C28, M14

Gobu, S. S26

Goldstein, D. PT-B8

Golubova, O.A. CP-B1

Gondal, R. CP-B4, PT-B3

Gopalakrishnaiah, S.S. S11

Gopinath M6

Govil, S. S22

Gubergrits, N.B. CP-B1, CP-B2, CP-C1, CP-C4, CP-C5, M1, M2, M3

Gukovskya, A. AP-B1

Guo, F. PT-B4, PT-C11, S17, S21, V6

Gupta, A.K., Lucknow PT-C18, S14, S23

Gupta, A.K., Thiruvananthapuram M20

Gupta, M., Madurai PT-C14, V4, V5

Gupta, M., New Delhi AP-C4

Gupta, R. AP-C17, AP-C18, AP-C19, AP-C21, AP-C22, CP-C13, CP-C26, M7, PT-C9, PT-C13

\section{KARGER}

(C) 2011 S. Karger AG, Basel and IAP

Fax +4161306 1234

E-Mail karger@karger.ch

www.karger.com

Accessible online at:

www.karger.com/pan 
Halagowder, D. PT-B10

Hamada, S. PT-B7

Hannah, A. M4

Haribhakti, S. AP-C3, V1

Harish, R. S11

Hastak, M. S9

Hayashi, H. PT-C2

Hickey, A. AP-B7

Hinduja, M.K. AP-C8, AP-C9

Hirota, M. PT-B7

Huang, W. AP-B1, AP-B2, AP-B8

Husain, S.A. CP-B4, PT-B3

Ikeura, T. CP-B7

Iyoob, V.A. CP-C17, PT-C5, S2

Jacob, M. AP-C32, V8

Jagannath, P. S9, S15, S27, V3

Jaipuria, J. AP-C45

Jat, A. AP-C51, PT-C6

Javaid, G. AP-C46

Jayant, M. M11

Jayanthi, V. AP-C42

Jeswanth, S. AP-C27, CP-C15, PT-C16, PT-C17, S20

Jeziorska, M. AP-B6

Jiang, K. PT-B6, PT-C11, S17, S21, V6

Johnson, C. M18

Johnson, M.A. M8, M9

Jose, F. CP-C23

Joseph, A.J. PT-C15

Joshi, K. CP-C26

Joshi, M. PT-C14, V4, V5

Jukemura, J. AP-B5, M19

Junejo, M.A. S7

Jupp, J. M18

Jyothibasu, D. AP-C27, CP-C15, PT-C17, S20

Jyotsna, V.P. M5

Kaistha, S. CP-C20, CP-C21

Kalra, N. M16

Kamalakannan, R. PT-C17

Kamalesh, N.P. V8

Kaman, L. CP-C26

Kang, M. AP-C17, AP-C18, AP-C19, AP-C21, AP-C22, M7

Kanno, A. PT-B7

Kapoor, A. PT-C3

Kapoor, S. PT-C8

Kapoor, V.K. PT-C18, S14, S23

Kar, P. CP-B4, PT-B3

Kar, S.K. CP-C25, CP-C27

Karanth, D. CP-C14

Kaur, R. AP-C29, M11

Kavalakkat, A.J. V11

Kawaguchi, M. PT-C1
Kawaguchi, Y. PT-C1

Khan, Md. A.G. M21

Khandelwal, N. AP-C22

Khare, M.K. S34

Kikuta, K. PT-B7

Kitamura, Y. PT-C2

Klymenko, A. CP-C6

Klymenko, V. CP-C6

Knyazeva, J. AP-C23

Kochhar, R.K. AP-C5, AP-C6, AP-C7, AP-C8, AP-C9

Koizumi, M. PT-C1

Koshy, A. CP-C33

Kotwal, V. AP-C14

Koyabu, M. CP-B7

Krishna, A. PT-C8, S12

Krishna, B. PT-C5

Kubrusly, M.S. AP-B5, PT-B11

Kumar, A., New Delhi AP-B4, CP-C18, PT-C8

Kumar, A., USA AP-C10

Kumar, H. CP-C35

Kumar, S. CP-C7

Kumaran, S. PT-B10

Kumaran, V. AP-C4

Kume, K. PT-B7

Kundil, B. PT-C26

Kurhade, S.N. PT-C14, V4, V5

Kurti, F. AP-C30

Kuruvilla, A.P. PT-C5

Kusuda, T. CP-B7

Kutyrkina, I.L. CP-C4

Lailaraji, N. PT-C5

Lakhia, R. AP-C25

Lalwani, S. AP-C4

Lampe, P. PT-B1

Lekha, V. AP-C41, CP-C30, CP-C31, CP-C32, S31, S32

Leonardi, G. M12

Levy, M.J. AP-C14

Levy, P. S6

Li, Q. PT-B6

Linevsky, Y.V. CP-C5

Lu, Z. PT-B6, PT-C11, S17, $\mathrm{S} 21, \mathrm{~V} 6$

Lukashevich, G.M. M2

Machado, M.C.C. AP-B5, AP-C34, M19, PT-B11

Madhav, T.V. PT-C12, S19, S24, S25

Madhavan, M. AP-C27, S20

Mahadevan, P. CP-C33

Mahesh, S. AP-C41, CP-C31, CP-C32, S31

Maheshwari, R. AP-C26

Maiwall, R. M22

Malecka-Panas, E. PT-B1

Malleo, G. PT-C19
Mallik, R.N. PT-C24

Mangla, V. S3

Manjuraj, K.P. CP-C29

Manoharan, G. S8

Manoj Kumar, A.P. AP-C33, S33, V10, V13

Marchi, S. M12

Masamune, A. PT-B7

Mascetta, G. PT-C19

Mason, J.M. AP-C2

Mathai, S.K. V9

Matsushita, M. CP-B7

Matthew, P. AP-C12

Maudar, K.K. PT-B13

Maydeo, A. AP-C12, CP-C9

McLaughlin, E. AP-B8

Meher, C. PT-C24

Mehta, N. AP-C4

Menon, R.N. AP-C40

Meyer, A.M. AP-B5

Miao, Y. PT-B4, PT-B5, PT-B6, PT-C11, S17, S21, V6

Michaelson, J.S. PT-C25

Midha, S. CP-C18, CP-C19

Midwinter, M. AP-C49

Miranda, C.J. AP-C2

Mishra, P.K. PT-B13

Mishra, S.R. AP-C1

Misra, B. CP-C25, CP-C27

Misra, M.C. PT-C8, S12

Miyoshi, H. CP-B7

Mizumoto, M. PT-C1

Mohan, N., Gurgaon M21

Mohan, N., Madurai PT-C14, V4, V5

Mohan, P. AP-C24

Mohapatra, M.K. PT-C24

Mohindra, S. AP-C31, AP-C35

Molan, N.T. AP-C34, M19

Monteiro-da-Cunha, J.E. AP-C34

Moorthy, S. S26

Mortele, K.J. AP-C38

Muddana, V. AP-C50

Mujumdar, N. PT-B9

Mukherjee, R. AP-B1, AP-B2, AP-B8

Murali Krishna, P. AP-C28, M14

Nada, R. CP-C26

Nadkarni, N. AP-C29, M11

Nagi, B. AP-C19, CP-C13, M7

Nagpal, A. AP-C3, V1

Nanda, M. AP-C19

Natesh, B. CP-C17, M20, PT-C5, S2

Nayak, P. PT-C24

Nihal, L. AP-C47

Nisar, A. AP-C46
Nishio, A. AP-B3, CP-B7

Nundy, S. AP-C4

Okada, T. PT-C2

Okazaki, K. AP-B3, CP-B7

Ola, R.P. CP-C11

Olakowski, M. PT-B1

Pal, S. CP-C20, CP-C21, M5, S3

Palaniswami, K.N. AP-C32

Palanivelu, C. AP-C33, S26, S33, V7, V10, V12, V13

Paliwal, S. CP-B3

Panackel, C. V9

Panda, C. CP-C25, CP-C27

Panigrahi, M.K. CP-C25, CP-C27

Pannala, R. PT-B2

Papachristou, G.I. AP-C50

Parker, M.J. S7

Patel, L. AP-C25

Patil, M. CP-C14, S11

Patil, R. M13

Patra, D.P. AP-C11, S4, S5

Patzina, R.A. AP-B5, AP-C34

Paul, S.C. AP-C47

Paulvannan, S. S13

Peethambaran, M.S. PT-C14, V4, V5

Petersen, O.H. AP-B8

Philip, S. AP-C37, AP-C43, V9

Phillips, A.R.J. AP-B7

Phillips, P. PT-B8, M15

Pirola, R. PT-B8, M15

Poddar, U. CP-C13

Polipalli, S.K. CP-B4, PT-B3

Pottakkat, B. PT-C18, S14, S23

Prakash, A. PT-C18, S14, S23

Prakash, K. AP-C32, S28, V8

Prasanna, K.S. CP-C14

Prathapan, V.K. S1

Praveenraj, P. AP-C33, S33, V10, V13

Puri, R. AP-C1

Qian, Z. PT-B4, PT-C11, S17, S21, V6

Raghavendra

Rao, R.V. AP-C26, PT-C20, PT-C21

Raghavendra, Y.B. AP-C21

Raghuram, G.V. PT-B13

Rai, P. AP-C31

Raj, M. AP-C35

Rajan, P.S. S26, S33, V7, V10, V12 
Rajan, R. CP-C17, M20, PT-C5, S2

Rajapandian, S. AP-C33, S33, V7, V10, V12

Rajarathinam, G. AP-C27

Rajegowda V8

Rajesh, G. CP-C35

Ramakrishna, B.S. M22

Ramanujam, A. AP-C33, S33, V10, V13

Ramesh, A. PT-C14, V4, V5

Ramesh, H. AP-C41, CP-C29, CP-C30, CP-C31, CP-C32, CP-C33, PT-C23, PT-C26, $\mathrm{S} 31, \mathrm{~S} 32$

Rana, S. AP-C18, CP-C26

Rana, S.S. AP-C17, AP-C18, AP-C19, AP-C20, AP-C21, AP-C22, CP-C11, CP-C13, M7, M16, PT-C9, PT-C10

Rana, S.V. CP-C11

Rao, G.V. CP-B3

Rao, R.V. CP-C16

Rasesh, S. M10

Rath, S. AP-C11, S4, S5

Rather, S. CP-C29

Raut, V. PT-C1

Ravi, R. AP-C27, PT-C17, S20

Ravichandran, P. AP-C27, CP-C15, PT-C17, S20

Ravikanth, K. M10

Ravindranath, K. AP-C26, AP-C44, PT-C20

Ray, G. AP-C48

Rebours, V. S6

Reddy Yeddula, P.K. AP-C47

Reddy, D.N. CP-B3

Reddy, J.R. PT-C18, S10, S14, S18, S23

Reddy, N. M6

Regimbeau, J.M. CP-C34

Reis, E.M. PT-B11

Ren, H. PT-B4

Rout, N. PT-C24

Roy, T.S. AP-B4

Ruszniewski, Ph. PT-C4

Sachdev, A. AP-C29, M11

Sagar, A. AP-C22

Sah, R. CP-B5, CP-B6

Sahni, P. CP-C20, CP-C21, M5, S3

Sahu, M. M22

Sakaguchi, Y. CP-B7

Salemi, V.M. AP-B5

Saluja, A. CP-B5, CP-B6, PT-B9, PT-B12

Salvia, R. PT-C19

Samantaray, S. PT-C24

Sampietre, S.N. AP-C34, M19
Sandeep, A. AP-C28

Sangeetha, M.K. M4

Sangwan, V. PT-B9, PT-B12

Sanyal, S. PT-C7

Saraf, V.A. S29

Saraswat, V. AP-C31, AP-C35

Saravanakumar V12

Saravanan, M.N. AP-C26, PT-C21

Sastry, R.A. CP-C12, M10, PT-C12, S19, S24, S25

Satoh, K. PT-B7

Satpudke, D. S9

Satyasree, B. AP-C47

Saumya, M. AP-C16, CP-C35

Sauvanet, A. PT-C4, S6

Saxena, R. PT-C18, S14, S18, $\mathrm{S} 23$

Sehgal, A. AP-C31

Sekwani, A. PT-C6, PT-B13, AP-C51

Selvakumar, E. AP-C27, CP-C15, PT-C17, S20

Senthilkumar, P. AP-C27, CP-C15, S20

Senthilnathan, P. AP-C33, S26, S33, V7, V10, V12, V13

Shabeerali, T.U. CP-C17, M20, PT-C5

Shah, R. V3, S9

Shah, R.G. S15, S27

Shaheen, F. AP-C46

Shaileshkumar, M.E. AP-C39

Shaji, P. AP-C51, PT-C6

Shalimar, S. CP-C18, CP-C19

Sharma, M., Cochin S31

Sharma, M., Meerut AP-C24

Sharma, R. M5

Sharma, S. CP-C11

Sheen, A.J. PT-C7, S7

Shenoy, K.T. CP-C24

Shenvi, S.D. PT-C13

Shetty, N. V2

Shimatani, M. CP-B7

Shimosegawa, T. PT-B7

Sidhu, R.S. CP-C13

Sihag, P. PT-C3

Sindhu, R.S. CP-C17, M20, PT-C5, S2

Singh, B. AP-C26, PT-C20, PT-C21

Singh, G., Chandigarh PT-C13

Singh, G., Kochi CP-C29, CP-C30, PT-C26

Singh, K. AP-C5, AP-C6, AP-C7, AP-C19, AP-C20, CP-C11, CP-C13, M7, M16, PT-C9, PT-C10
Singh, R. AP-C8, AP-C9, AP-C17, AP-C18, AP-C21, PT-C13

Singh, R.K. PT-C3, PT-C18, $\mathrm{S} 10, \mathrm{~S} 14, \mathrm{~S} 23$

Singh, S.P. CP-B3, CP-C25, CP-C27, PT-C24

Singh, V. PT-C6, AP-C51

Singh, V.K. AP-C38

Singla, P. AP-C4

Sinha, S.K. AP-C19, CP-C13

Siriwardena, A.K. AP-B6, AP-C2, AP-C36, PT-C7, S7

Sivasubramaniam, B. AP-C42

Smolarz, B. PT-B1

Solanki, R.P. S24

Soni, H. AP-C3, V1

Srikanth Reddy, Y. AP-C47

Srinivas, N. AP-C28, M14

Srinivasan, R. M16, PT-C9

Srinivasan, U.P. S8

Srinivasan, V. AP-C42

Sriram, P. M6

Subhalal, N. PT-C5

Subhash, R. CP-C17, PT-C5

Sud, R. AP-C1

Sudheer, O.V. AP-C39, AP-C40, S29

Sudhindran, S. AP-C39, AP-C40, S29

Sukumar, C. PT-C17, S20

Sukumar, R. AP-C27, CP-C15, PT-C17

Sukumar, S. AP-C27

Suman Bhushan, K. M10, S25

Sundaram, K.R. CP-C10

Sunil Kumar, K. CP-C23

Surendran, R. AP-C27, AP-C42, CP-C15, PT-C16, $\mathrm{PT}-\mathrm{C} 17, \mathrm{~S} 20$

Sutariya, K.G. S15, S27

Sutton, R. AP-B1, AP-B2, AP-B8

Sylesh, A. V8

Tahira, A.C. PT-B11

Takaoka, M. CP-B7

Takaori, K. PT-C1

Talar-Wojnarowska, R. PT-B1

Talukdar, R. AP-C13, AP-C14, AP-C15

Tampi, C.S. S9

Tanaka, H. CP-C8

Tandon, N. M5

Tank, A.K. PT-C18, S23

Tepikin, A. AP-B2, AP-B8

Thandassery, R.B. AP-C5, AP-C6, AP-C7

Thapa, B.R. CP-C13, M7

Thayer, S.P. PT-C22, PT-C25
Thomas, V. CP-B3, CP-C23

Tiwari, N.A. AP-C39, S30

Tranchart, H. S6

Trehan, S.S. M17

Uchida, K. AP-B3, CP-B7

Uemoto, S. PT-C1

Unno, J. PT-B7

Unno, M. PT-C2

Uppin, S.G. PT-C12

Vaitheeswaran, V. S26, S33, V7, V10, V12

Valsangkar, N.P. PT-C22, PT-C25

Varghese, C.J. V11

Varghese, K. S11

Varma, D. AP-C32, V9

Varma, V. AP-C4

Varshney, R. AP-C51, PT-B13, PT-C6

Varshney, S. AP-C51, PT-B13, PT-C6

Vasanthi, R. M4

Vasudev, C. AP-C35

Vatsal, S. S4

Vaze, S. V3

Veena, A.B. CP-C10

Vege, S.S. AP-C10, AP-C13, AP-C14, AP-C15

Venkataraman, J. PT-B10

Venkatarami Reddy, V. CP-C16

Venu, N. M6

Venugopal, A. CP-C32, PT-C23, S31

Venugopal, B. AP-C41, CP-C29, CP-C30, CP-C32, PT-C23, S31, S32

Venugopal, Y.C. S11

Verjovski-Almeida, S. PT-B11

Vickers, S.M. PT-B9, PT-B12

Vij, A. V12

Vikram, G. S13

Vimalraj, V. AP-C27, CP-C15, PT-C16, PT-C17, S20

Vipin, I.S. V8

Vonlaufen, A. M15

Voronin, K.A. M3

Vullierme, M.P. S6

Vyas, H.G. AP-C45

Wagh, Y. S13

Waiz, G. AP-C46

Wang, F. PT-B5

Wang, H. S16

Wani, M. CP-C32, PT-C26, S32

Warshaw, A.L. PT-C22, PT-C25

Wei, J. PT-B4, PT-B5, PT-C11, S17, S21, V6 
Welchman, S.A. AP-C49

Whitcomb, D.C. AP-C50

Wilkins, A. AP-C49

Wilson, J. PT-B8, M15

Wilson, M. AP-C49

Wu, J. PT-B4, PT-B5,

PT-C11, S17, S21, V6
Xu, Z., Australia PT-B8, M15

$\mathrm{Xu}, \mathrm{Z}$., China PT-B4, PT-C11, S17, S21, V6

Xue, X. PT-B5, PT-B6

Yadav, A. AP-C4
Yadav, T.D. AP-C5, AP-C6, AP-C7, AP-C8, AP-C9, CP-C26

Yang, L. PT-B8, M15

Yattoo, G.N. AP-C46

Yoshida, K. CP-B7

Youkhana, J. M15
Yurjeva, A. CP-C1

Zaimi, E. AP-C30

Zamboni, G. PT-C19

Zargar, S.A. AP-C46

Zhang, Y. PT-B4 